\title{
Positron Annihilation in Simple Condensed Gases
}

by

John James Steinbacher

A thesis submitted to the Department of Physics in conformity with the requirements for the degree of Doctor of Philosophy

\author{
Queen's University \\ Kingston, Ontario, Canada
}

September 1998

Copyright (c) John James Steinbacher, 1998. 
National Library

of Canada

Acquisitions and

Bibliographic Services

395 Wellington Street Ottawa ON K1A ON4

Canada
Bibliothèque nationale

du Canada

Acquisitions et services bibliographiques

395. rue Wellington Ottawa ON K1A ON4

Canada
The author has granted a nonexclusive licence allowing the National Library of Canada to reproduce, loan, distribute or sell copies of this thesis in microform, paper or electronic formats.

The author retains ownership of the copyright in this thesis. Neither the thesis nor substantial extracts from it may be printed or otherwise reproduced without the author's permission.
L'auteur a accordé une licence non exclusive permettant à la Bibliothèque nationale du Canada de reproduire, prêter, distribuer ou vendre des copies de cette thèse sous la forme de microfiche/film, de reproduction sur papier ou sur format électronique.

L'auteur conserve la propriété du droit d'auteur qui protège cette thèse. Ni la thèse ni des extraits substantiels de celle-ci ne doivent être imprimés ou autrement reproduits sans son autorisation. 


\section{Abstract}

Conventional angular-correlation experiments were performed in several simple condensed media to study the effects of various physical and chemical processes on positronium (Ps) yields. Momentum spectra were obtained with static magnetic fields applied to liquid helium, neon, argon, and nitrogen and to solid neon and argon. Experiments with electric fields applied and with $\mathrm{O}_{2}$ impurity deliberately added were also done in liquid argon and $\mathrm{N}_{2}$. All of the results show at least two momentum components: a narrow component due to para-positronium self-annihilations from a localized bubble state; and a broad component due to positron and Ps pick-off annihilations.

Apart from liquid helium, the existing theoretical estimates for the positron broad component were not good enough to allow a reasonable separation of the broad and narrow components. Realistic positron-rare gas potentials were generated and used to calculate the positron band structure and wave functions in the heavier rare gas solids. The positron momentum distributions and lifetimes were then calculated taking proper account of the positron-core electron correlations. The resulting predictions are in good agreement with experiment.

The measurements in liquid helium, neon, argon and $\mathrm{N}_{2}$ in zero magnetic field all show a narrow component that is well described by the spherical square-well bubble model. Both the predicted shape of the narrow component and the bubble radius are in good agreement with experiment. In liquid helium, the increases seen in the narrow component intensity with magnetic field follow theoretical predictions. A fit to the Ps yield in liquid neon as a function of magnetic field gives an estimate of 4.8-6.5 ns for the long-lived orthopositronium lifetime. The magnetic field effect in liquid argon was surprisingly small. It is expected that another quenching mechanism, spin-exchange conversions between para and ortho-positronium induced by either $\mathrm{O}_{2}$ impurity or free electrons in the positron spur, is competing with the magnetic field. A simple theoretical model was developed to describe the magnetic and spin-exchange quenching.

The electric field and $\mathrm{O}_{2}$ impurity experiments in liquid argon and $\mathrm{N}_{2}$ provide useful insight in to the Ps formation process. Liquid $\mathrm{N}_{2}$ shows a significant decrease in Ps yield with electric field. The effect in liquid argon is much smaller. In both liquids, the $\mathrm{O}_{2}$ 
impurity data shows a sharp increase in the narrow component at low $\mathrm{O}_{2}$ concentrations, followed by a maximum and a gradual decrease at higher impurity concentrations. These results are explained in terms of the usual Ps formation models, positron thermalization times and the possible positron spur interactions. 


\section{Statement of Originality}

This thesis is a completely original work. This work was performed either independently or in collaboration with Professor Alec Stewart who is duly credited in the acknowledgments. The ideas, work and results of other authors are presented and used throughout this thesis. In all cases, their work is acknowledged, referenced and proper credit given. 


\section{Acknowledgments}

I wish to express my sincere gratitude and appreciation to Professor Alec Stewart for his supervision and guidance during this research project. His knowledge, wisdom and expertise helped to make this a most satisfying learning experience. His enthusiasm, patience and kindness made it an enjoyable one.

I would also like to thank the many good people at the Chalk River Laboratories who helped with the copper irradiations required for the positron sources. These include Dr. Bill Buyers, head of the Neutron and Solid State Physics Division, who financially supported the irradiations. I am also indebted to Dr. Bill Edwards and Vic Corriveau of the General Chemistry Division who organized each of the irradiations and prepared the sources for shipment to Kingston.

I am very grateful to all those people in the department who shared their expertise and equipment. These include Dr. Barry McKee with whom I enjoyed many interesting and enlightening discussions. It is also a pleasure to acknowledge the many hours of discussion and help provided by my lab mates and friends Andrew Duffy, Greg Hiltz, and Paul Capes. Finally, I must also thank Jim Thompson and Terry Bovington of the machine shop for their help in constructing assorted bits of the apparatus. 


\section{Dedication}

To my family,

for their love and support. 


\section{Table of Contents}

Abstract

Statement of Originality $\quad$ iii

Acknowledgments $\quad$ iv

Dedication $\quad$ v

Table of Contents vi

List of Tables $\quad$ x

List of Figures and Illustrations $\quad$ xi

1 Introduction 1

2 Introduction to Positron Physics 5

2.1 Introduction . . . . . . . . . . . . . . . . 6

2.2 The Creation and Physical Properties of Positrons . . . . . . . . . 6

2.3 Positron Thermalization ....................... 8

2.3.1 The Slowing-Down Process ............... 8

2.3.2 The Implantation Profile .................... 12

2.3.3 The Positron Spur .................... 14

2.4 Positron States in Condensed Matter . . . . . . . . . . . 15

2.4.1 Delocalized Positron Wave Functions . . . . . . . . . . 16

2.4.1.1 The Wigner-Seitz Approximation ........... 1 . .

2.4.1.2 The Augmented Plane Wave Method . . . . . . . . 18

2.4.1.3 The Pseudopotential Method ............ 21

2.4.2 Localized Positron States ... . . . . . . . . . . . 24

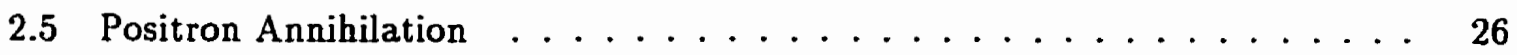

2.5.1 The Annihilation Event ..................... 27

2.5.2 $2 \gamma$ Annihilation in Condensed Matter ............ 31

2.5.3 Core-Electron Annihilation ............... 33 
2.5.4 Positron-Core Electron Correlations .............. 35

3 Positronium Physics and Chemistry $\quad 40$

3.1 Properties of an Isolated Positronium Atom .............. 41

3.2 Positronium in Condensed Matter ................. 43

3.3 Positronium Formation Models . . . . . . . . . . . . . . . 46

3.4 Positronium States in Condensed Matter . . . . . . . . . . . . . 51

3.4.1 Delocalized Positronium States ............... 52

3.4 .2 The Positronium Bubble State ............... 52

3.4.3 Trapping at Defects in Solids ................ 60

3.4 .4 Positronium Bound States . . . . . . . . . . . . 62

3.5 The Inhibition and Quenching of Positronium . . . . . . . . . 65

3.5.1 Magnetic Field Effects . . . . . . . . . . . . . . 65

3.5 .2 Electric Field Effects . . . . . . . . . . . . . . 68

3.5.3 Spin-Exchange Conversion . . . . . . . . . . . 70

3.5.3.1 Ps Conversions With Spur Species . . . . . . . . . 70

3.5.3.2 The Effects of Oxygen Impurity ........... 75

4 The 1D-ACAR Experiments and Results $\quad 79$

4.1 The ACAR Experiments ............................. 79

4.1 .1 The 1D-ACAR Apparatus ..................... 80

4.1.2 The Sample and Source Arrangement ............. 82

4.1.3 2D-ACAR Experiments .................... 86

4.2 Previous ACAR Results . . . . . . . . . . . . 86

4.3 1D-ACAR Results and Analysis . . . . . . . . . . . 89

4.3.1 Data Corrections and Initial Analysis ............ 90

4.3 .2 Experimental Results ................... 93

4.4 Positron Annihilation Lifetime Spectroscopy . . . . . . . . . . . . 109

4.5 Doppler-Broadening Experiments . . . . . . . . . . . . 115

4.6 Slow Positron Beams . . . . . . . . . . . . . . . 116

5 The Broad Momentum Component $\quad 118$

5.1 Previous Theoretical Estimates .................. 119

5.1.1 Positron-Gas Scattering Results ............... 119 
5.1 .2 Solid-State Calculations . . . . . . . . . . . . . . 123

5.2 Possibility of Intermediate Momentum Components . . . . . . . . 125

5.3 Calculation of the Broad Momentum Component . . . . . . . . . . . 127

5.3.1 Positron Potentials ...................... 127

5.3 .2 Positron Energy Levels ................... 131

5.3.3 Positron Wave Functions .................... 137

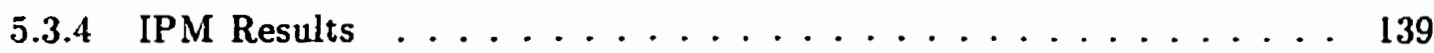

5.3.5 Effects of Positron-Core Electron Enhancements . . . . . . . 142

6 The Narrow Momentum Component 150

6.1 Positronium Potentials and Delocalized Energies . . . . . . . . . 150

6.2 Zero-Field Positronium Formation ................ 154

6.3 Positronium Bubble Model Results . . . . . . . . . . . . 158

6.4 Magnetic Field Effects . . . . . . . . . . . . . . 165

6.5 Electric-Field Effects . . . . . . . . . . . . . . . 166

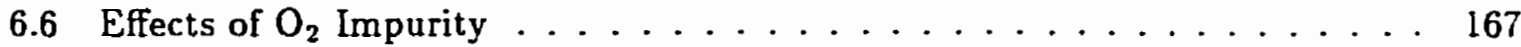

6.7 Spin-Exchange Model Calculations . . . . . . . . . . . 168

7 Conclusions $\quad 172$

$\begin{array}{lr}\text { Bibliography } & 175\end{array}$

$\begin{array}{ll}\text { A Properties of the Condensed Gases } & 190\end{array}$

$\begin{array}{ll}\text { B Positron Properties } & 195\end{array}$

C Thermalization Times in the Condensed Rare Gases 202

D Extended Self-Trapped Positron States $\quad 210$

E Positronium Spin-Exchange Cross-Sections 212

F The 1D-ACAR Experimental Resolution $\quad 216$

G The Löwdin Alpha-Function Expansion $\quad 218$

H Calculation of the Solid-State Potentials $\quad 220$ 
Curriculum Vitae 


\section{List of Tables}

2.1 Summary of $\mathrm{e}^{-}-\mathrm{e}^{+}$annihilation characteristics $\ldots \ldots \ldots \ldots$

3.1 Effects of $\mathrm{O}_{2}$ impurity on the positron spur $\ldots \ldots \ldots 78$

4.1 A summary of experimental angular-correlation results . . . . . . . 88

4.2 Parameters for the two Gaussian fits to the broad component . . . . . . 9 91

4.3 A summary of positron lifetime results . . . . . . . . . . L14

5.1 Results of the positron band gap calculations $\ldots \ldots \ldots \ldots$

5.2 Average positron-core electron enhancement factors . . . . . . . . . . 143

6.1 Positronium-rare gas Lennard-Jones potential parameters . . . . . . . . 152

6.2 Summary of zero-field narrow peak measurements . . . . . . . . . . . 155

6.3 Summary of Ore gap formation calculations . . . . . . . . . . $15 i$

6.4 Square well bubble model results . . . . . . . . . . . . . 159

A.1 A summary of the atomic rare-gas properties . . . . . . . . 191

A.2 A summary of the diatomic-gas properties . . . . . . . . . . 192

A.3 A summary of liquid rare-gas properties . . . . . . . . . . . . 192

A.4 A summary of the diatomic liquid properties $\ldots \ldots \ldots \ldots$

A.5 Summary of the solid properties ... . . . . . . . . . 194

B.1 Physical properties of the positron ............... 196

B.2 Characteristics of common positron-emitting radioisotopes . . . . . . 196

C.1 Electron thermalization times .................. 203

C.2 High-energy positron thermalization times .............. 205

C.3 Low-energy positron thermalization times . . . . . . . . . . 208

C.4 Ps thermalization times . . . . . . . . . . . . . . 209

H.1 Parameters used in the electron and positron potentials . . . . . . . . 224 


\section{List of Figures and Illustrations}

2.1 Positron processes in condensed matter $\ldots \ldots \ldots . \ldots . \ldots$

2.2 The positron ionization trail . . . . . . . . . . . . . 12

2.3 Two-photon annihilation . . . . . . . . . . . . 30

4.1 ID Angular-correlation apparatus . . . . . . . . . . . . . 80

4.2 Previous angular-correlation spectra $\ldots \ldots \ldots \ldots$

4.3 1D-ACAR data in liquid $\mathrm{He} \ldots \ldots \ldots \ldots . \ldots . \ldots . \ldots . \ldots$

$4.4 I_{n}$ versus magnetic field for $\mathrm{He}$ and $\mathrm{Ne} \ldots \ldots \ldots . \ldots . \ldots 5$

4.5 1D-ACAR data in liquid $\mathrm{Ne} \ldots \ldots \ldots \ldots . \ldots \ldots$

4.6 1D-ACAR data in solid $\mathrm{Ne} \ldots \ldots \ldots \ldots . \ldots \ldots$

4.7 1D-ACAR data in liquid Ar in various magnetic fields . . . . . . . . 99

4.8 1D-ACAR data in solid Ar in zero and high magnetic fields . . . . . . 100

$4.9 I_{n}$ versus magnetic field for liquid Ar .............. 10 I

$4.101 \mathrm{D}$-ACAR data in liquid Ar with $\mathrm{O}_{2}$ impurity present $\ldots \ldots \ldots$

$4.11 I_{n}$ versus $\mathrm{O}_{2}$ concentration in liquid $\mathrm{Ar}$ and $\mathrm{N}_{2} \ldots \ldots \ldots$

4.12 ID-ACAR data in liquid $\mathrm{N}_{2}$ in various magnetic fields ........ 105

$4.13 I_{n}$ versus magnetic field for liquid $\mathrm{N}_{2} \ldots \ldots \ldots \ldots \ldots$

4.14 1D-ACAR data for liquid $\mathrm{N}_{2}$ with $\mathrm{O}_{2}$ impurity present $\ldots \ldots \ldots 108$

4.151 D-ACAR data for liquid $\mathrm{N}_{2}$ in various electric fields . . . . . . 110

$4.16 I_{n}$ versus electric field in liquid $\mathrm{Ar}$ and $\mathrm{N}_{2} \ldots \ldots \ldots \ldots$

4.17 Schematic diagram of a positron lifetime spectrometer . . . . . . . 112

5.1 Previous broad component calculations in $\mathrm{H}_{2}$ and helium $\ldots \ldots \ldots$

5.2 Previous broad component calculations in the heavier rare-gas solids . . . 122

5.3 Positron potentials in the rare-gas solids ............... 130

5.4 Positron band structure for the rare-gas solids . . . . . . . . . . . 132

5.5 Positron ground-state energy in the condensed rare gases $\ldots \ldots \ldots$

5.6 Positron wave functions in the rare-gas solids $\ldots \ldots \ldots 137$

5.7 Positron pseudopotential wave functions in the solid argon . . . . . . 138

5.8 IPM broad momentum components in the rare-gas solids . . . . . . . . 140

5.9 IPM lifetimes for positrons in the condensed rare gases . . . . . . . . 141 
5.10 Momentum dependent enhancements . . . . . . . . . . . . 145

5.11 Calculated broad momentum components in the condensed rare gases . . 147

5.12 Calculated lifetimes for positrons in the condensed rare gases . . . . . . 148

6.1 Dependence of Ps-atom scattering length versus $\sigma \ldots \ldots \ldots \ldots$

6.2 Free positronium energy in condensed $\mathrm{He}, \mathrm{Ne}$ and $\mathrm{Ar} \ldots \ldots . . . . .153$

6.3 Ps bubble radius as a function of well-depth. . . . . . . . . 160

6.4 Ps in a bubble overlap with the medium. . . . . . . . . . . 161

6.5 The narrow component in $\mathrm{He}, \mathrm{Ne}, \mathrm{Ar}$ and $\mathrm{N}_{2} \ldots \ldots \ldots$

$6.6 I_{n}$ versus magnetic field for liquid Ar with spin-conversion collisions $\ldots \ldots 170$

B.1 Decay modes for ${ }^{22} \mathrm{Na}$ and ${ }^{64} \mathrm{Cu} \ldots \ldots \ldots \ldots \ldots \ldots$ 


\section{Chapter 1}

\section{Introduction}

The study of positron annihilation has had, and continues to have, a significant impact on a diverse range of fields in physics and chemistry. A survey of the more recent international positron annihilation conferences shows that the field deals with theory and experiments in quantum electrodynamics, weak interactions and atomic physics, with many aspects of solid state physics, with surface, interface and thin film studies, with the physics and chemistry of gases and liquids, and even with some biophysics and biochemistry, including some medical imaging applications. This thesis focused on studying and understanding some of the simplest condensed matter systems.

Positron annihilation techniques have proven themselves to be powerful methods in the study of condensed matter. Since the early results of the 1950's a wide variety of positron annihilation experiments have been performed in a broad range of materials including metals and alloys, both normal and with defects, semiconductors, insulators, polymers and more recently high- $T_{c}$ superconductors and buckeyballs. Numerous sophisticated theoretical models have also been developed to both describe and predict these experimental results. However, despite the many successes of positron annihilation techniques, many important and interesting problems within the field remain unresolved. The motivation behind this thesis is to obtain a better understanding of some of these problems by studying several simple insulating materials, namely, the condensed rare and diatomic gases.

To appreciate the problems being addressed it is useful to first have a brief, qualitative description of what happens when a positron enters a condensed insulating material. In most experiments, positrons are obtained from the $\beta$ decay of certain radioactive nuclei and therefore have a broad energy spectrum on the order of several hundred $\mathrm{keV}$. Once the positron enters into the sample it begins to lose its initial high energy through excitations and ionizations in the medium. The trail of free electrons, ions, excited atoms and other species left by the positron is usually referred to as a radiation spur. Within picoseconds the positron's energy has been reduced to several tens of $\mathrm{eV}$, its motion has become more diffusive and the terminal part of the radiation spur, called the positron spur, has formed. The positron spur can crudely be thought of as a positron in the centre of a spherical dis- 
tribution of spur species (ions, free electrons, etc.). Annihilation during the slowing down process is rare. From this time onward there are, in general, several different modes of thermalization and eventual annihilation. The positron may simply continue slowing down until it reaches thermal energies, polarizing and interacting with the medium and eventually annihilating with either a free spur electron or with one bound to an atom. In insulating media, some fraction of the positrons will form a bound, hydrogen-like state with an electron called positronium (Ps). In the Ore gap Ps formation model the positron captures an electron from an atom during a collision. The spur formation model suggests that the positron combines with one of the free electrons created along the ionization trail. In most insulating liquids Ps formation is followed by a rather spectacular phenomenon - the selftrapping of Ps into a 'bubble' state. The lowest energy state of the system is one in which the Ps minimizes the exchange repulsion between its electron and the medium by creating and eventually annihilating from inside a cavity in the medium. The lack of 'free volume' in the interstitial regions of the rare-gas solids makes delocalized Ps unlikely. Instead the Ps is expected to either form inside or trap into a pre-existing defect. The eventual annihilation of positrons and Ps typically produces two $511 \mathrm{keV} \gamma$-rays. By detecting the $\gamma$-rays it is possible to measure the average positron and Ps lifetimes and the momentum distribution of the electron-positron pair. These results not only reflect the properties of the sample but also the behaviour of the positrons and Ps.

The experiments reported in this thesis were performed using the conventional angular-correlation technique to measure the momentum distribution of the annihilating electron-positron pair. The experiments were performed in liquid helium, neon, argon and nitrogen and in solid neon and argon. The angular-correlation spectra typically show at least two moment um components. The first is a narrow momentum component that results from singlet Ps self-annihilating from the bubble state in liquids, or, as will be shown, from pre-existing defects in solids. This narrow component is then superimposed on top of a much broader momentum component. The width of this broad peak is comparable to the electron momentum distribution of the medium and is therefore attributed to positrons annihilating with core electrons and to Ps pick-off annihilations (i.e. the positron in the Ps annihilates with an electron other than its own). The aim of the thesis was to measure the effects of various physical and chemical process on these distributions. To this end, angularcorrelation experiments were performed for the first time with static magnetic fields applied to the samples. Experiments with static electric fields applied and with small amounts of $\mathrm{O}_{2}$ 
impurity deliberately added were also done in liquid argon and $\mathrm{N}_{2}$. The broad component showed little change throughout these experiments. However, the effects on the narrow Ps momentum component were significant and sometimes dramatic.

In order to study the effects of various physical and chemical processes on Ps it is necessary to separate the broad and narrow components. In helium the narrow peak is suffciently different from the broad component that this separation is relatively easy. Previous theoretical calculations of the helium broad component are also of high enough quality to be useful in this separation. However, this was not the case in the heavier condensed media where the wider narrow components make the separation from the broad component far more difficult. Furthermore, the existing theoretical estimates are consistently too wide and of little use in separating the components. If the existing estimates are assumed to be correct, then they imply the existence of other intermediate momentum components whose width is in between the broad and narrow components. However, it might also be that these estimates are not yet adequate. More specifically, it is expected that a proper account needs to be taken of the annihilation enhancement factors that arise from the positron-core electron correlations.

The thesis began as a study of the dynamics of Ps bubble formation. It was hoped that if the time scale for Ps bubble formation was comparable to its lifetime, then different magnetic fields could be used to change the Ps lifetime and observe the bubble at different times during its growth. However, it appears that the Ps bubble is formed and comes to equilibrium on a time scale that is short compared to the Ps lifetimes. The experiments did, however, show an anomalous magnetic field effect in liquid argon. The results suggested that some quenching or spin-exchange process was occurring in liquid argon that did not occur in liquid helium and neon. The most likely cause of the anomaly was Ps spin-exchange collisions with either free electrons in the positron spur or with $\mathrm{O}_{2}$ impurity. Electric fields were applied to liquid argon and $\mathrm{N}_{2}$ to see what the effects would be on Ps formation and on the possible spin-exchange collisions with spur electrons. These results provide a simple resolution to the controversy over whether the Ore gap or the spur Ps formation model is correct in condensed media. Small amounts of $\mathrm{O}_{2}$ impurity were also added to liquid argon and $\mathrm{N}_{2}$ to help determine if it was the cause of the Ps spin-exchange conversions. The $\mathrm{O}_{2}$ had a dramatic effect on the observed narrow peak intensities that can be qualitatively understood in terms of the two Ps formation models, positron thermalization times and various positron spur interactions. 
The thesis starts with a discussion of some of the introductory concepts and details required to understand the results presented in this work. Chapter 2 focuses on describing the thermalization and eventual annihilation of a positron inside a condensed material. This includes a description of theories and techniques that can be used to calculate the positron energies, wave functions, lifetimes and annihilation momentum distributions. Chapter 3 describes the interesting and often complicated history of Ps immersed in condensed matter. This includes a discussion of the Ps formation models, a simple square-well model used to describe the self-trapped Ps bubble state, and the effects of magnetic and electric fields and spin-exchange collisions on Ps. The experimental apparatus and procedure used to measure the $2 \gamma$ angular distributions is described in Chapter 4 . The experimental results in each of the media studied are presented and summarized. Summaries of relevant angular correlation and positron lifetime results from other authors are also given. Chapter 5 is concerned with understanding and analyzing the broad components. This chapter begins with a summary of the relevant results from previous authors. The possibility that other intermediate' momentum components exist is discussed. Positron potentials and wave functions are calculated and used with more recent positron-core electron annihilation enhancements to obtain improved theoretical estimates of the broad momentum component and positron lifetimes. The narrow momentum component is studied and analyzed in Chapter 6. The simple bubble model is used to analyze the data. The Ps formation fraction, both with and without electric and magnetic fields applied and with $\mathrm{O}_{2}$ impurity added, is discussed. A model that describes the magnetic-field effects in the presence of spin-exchange collisions is applied to the anomalous results in liquid argon. Conclusions are drawn in Chapter 7. 


\section{Chapter 2}

\section{Introduction to Positron Physics}

In this thesis, positron-annihilation experiments were performed in which energetic positrons from a radioactive source were injected into simple condensed gases. Once in the sample, the positrons generally slow down to thermal energies in a very short time ( $\sim 1 \mathrm{ps})$. Their mean implantation depth of $10-1000 \mu \mathrm{m}$ ensures that the positrons sample the bulk material. Once thermalized, the positrons exist in delocalized Bloch states until they eventually annihilate with an electron from the surrounding medium - typically producing two $511 \mathrm{keV} \gamma$-rays. Useful information, such as the average positron lifetime and the momentum distribution of the annihilating electron-positron pair, can be obtained by detecting these $\gamma$-rays. The average positron lifetime is characteristic of each material, varying from 100-500 ps, and provides a measure of the electron density seen by the positron. The aim of this chapter is to provide the details necessary to understand these processes and the experimental results arising from annihilating positrons. In insulating condensed media some fraction of the positrons may form positronium during the slowing down process. The interesting and often complicated history of positronium will be the sub-

ject of chapter 3. Much of the material presented in these next two chapters is common to many positron-annihilation studies. Further details can be found in several of the excellent books (Hautojarvi 1979; Brandt and Dupasquier 1983; Schrader and Jean 1988), reviews (Berko and Hereford 1956; Ferrell 1956; Wallace 1960; Goldanskii 1968; and West 1973) and conference proceedings.

This chapter begins with a brief description of the positron, its properties and its creation in $\$ 2.1$ and $\S 2.2$. Further details are given in Appendix B. The mechanisms by which energetic positrons slow down in condensed matter are described in $\$ 2.3$. This includes a discussion of the ionization trail and 'positron spur' which become important considerations later on. The possibility that positrons do not reach thermal equilibrium before annihilating in the condensed rare gases is explored in Appendix C. Section 2.4.1 describes several techniques that are used to calculate the energies and wave functions for thermal Bloch state positrons. Using these wave functions it is possible to calculate both the positron lifetime and the annihilating pair momentum distribution. The annihilation 
process is described in $§ 2.5$. This will include a discussion of the many-body positronelectron correlations which significantly affect the annihilation characteristics. Whenever possible, the discussion will focus on the condensed rare and diatomic gases of interest to this work. Appendix A contains a summary of the properties useful to this thesis for the rare gases and $\mathrm{H}_{2}, \mathrm{~N}_{2}$ and $\mathrm{O}_{2}$.

\subsection{Introduction}

The conceptual origin of the positron can be traced to Dirac's (1930) relativistic treatment of quantum mechanics. The solution to his relativistic wave equation for a free electron, of mass $m_{e}$ and momentum $\mathbf{p}$, results in an energy spectrum $E= \pm \sqrt{\mathbf{p}^{2} c^{2}+m_{e}^{2} c^{4}}$ that consists of two continuous bands separated in energy by an interval $2 m_{o} c^{2}$, where $c$ is the speed of light. The negative-energy solutions to Dirac's equation produced an energy spectrum that was no longer bounded from below and implied the seemingly unphysical situation that the positive-energy states were unstable. To overcome this it was postulated that in the 'vacuum' the negative-energy states were completely filled by an infinite sea of unobservable negative-energy electrons. Pauli exclusion ensured the stability of the positiveenergy states by preventing the positive-energy electrons from falling into the negativeenergy states. Given enough energy, say by absorbing a photon with $\hbar \omega>2 m_{e} c^{2}$, an electron in the negative-energy sea could be excited into an observable positive-energy state leaving behind a 'hole' in the negative-energy sea. This hole makes the negative-energy sea less negative in both energy and charge, compared to the original vacuum. The hole therefore appears as a positive energy, positively charged particle - i.e. a positron $\left(\mathrm{e}^{+}\right)$. Within this framework, the annihilation of an electron with a positron can be viewed as a positive-energy electron de-exciting back into a hole in the negative-energy sea, with the energy typically being given off as $\gamma$-rays. The positron's existence was verified soon after Dirac's prediction by Anderson (1932a, 1932b, 1933) while performing cloud chamber experiments on cosmic rays.

\subsection{The Creation and Physical Properties of Positrons}

The positron is a stable elementary particle $\left(\tau>2 \times 10^{22}\right.$ years for an isolated positron (Bellotti et al. 1983)) which, like electrons, muons, taus and neutrinos ( $\nu$ ), belongs 
to the lepton family. As leptons, positrons are fermions which obey Fermi-Dirac statistics and do not participate directly in the strong interaction. The CPT theorem, which requires that a system be invariant under a transformation in which spatial inversion $(P)$ and time reversal $(T)$ are accompanied by charge conjugation $(C)$, implies that particles and antiparticles must have the same rest mass, and that their electric and magnetic moments and spin vectors differ only in sign. As the electron's antiparticle, the positron has the same rest mass and spin $(s=1 / 2)$ but opposite charge $(+e)$ and lepton number $(-1)$. A comparison of the magnitudes of electron and positron properties in Table B.1 shows that they are identical to within the rather impressive accuracies achieved so far. In fact, measurements of the $g$-factor anomaly by Van Dyck et al. (1987) are the most sensitive tests of the particle-antiparticle symmetry predicted by $C P T$ invariance and one of the most stringent tests of quantum-electrodynamic (QED) calculations. The difference in charge implies that while positrons must obey the Pauli exclusion principle with respect to each other, there is no Pauli exclusion between an electron and a positron.

Positrons do not exist naturally on earth except for short times and in relatively small numbers. They are created by various nuclear reactions including pair production and radioisotope decay. They also occur as one of the decay products of various unstable particles, such as the positive muon. Most positron-annihilation experiments use positrons emitted from neutron-deficient radioisotopes. There are more than 200 positron-emitting nuclides, of which only about a dozen are useful as positron sources. The characteristics of the common radioisotopes used in positron experiments are summarized in Appendix B. The positron $\beta$-decay process, in which a proton of the nucleus is transformed into a neutron $\left(p \rightarrow n+\mathrm{e}^{+}+\nu\right)$, is very similar to the $\beta^{-}$-decay of nuclides having a neutron excess $\left(n \rightarrow p+\mathrm{e}^{-}+\bar{\nu}\right)$. In both processes the nuclide decays by emitting particles with a simple continuous energy spectrum, typically from several hundred $\mathrm{keV}$ to several $\mathrm{MeV}$ wide, with a cutoff at an energy $E_{\max }$. The interaction of the particle with the Coulomb potential of the nucleus reduces the low-energy portion of the $\beta^{+}$spectrum, relative to the $\beta^{-}$spectrum, and so the average initial positron energy $\left(E_{\text {ave }} \sim 0.4 E_{\max }\right)$ is higher than the average electron energy $\left(\sim 0.3 E_{\max }\right)$. Positrons emitted in radioisotope decay are highly spin polarized since parity is not conserved in this weak-interaction process. The degree to which the positron's spin is oriented parallel to its momentum is $v / c$, where $v$ is the positron's speed. 


\subsection{Positron Thermalization}

In between their creation and eventual annihilation, positrons have an interesting and complicated history. Some of the typical processes that may occur in condensed insulating media are shown in Figure 2.1. A natural starting point for a discussion of the positron's history in condensed matter is the slowing-down, or thermalization, process. These processes are very similar to those for energetic electrons, despite a number of important differences - the sign of the charge, the absence of exchange effects, and the possibility of Ps formation and annihilation. This means that with some care, much of the understanding that radiation chemists and physicists have achieved concerning the slowing down of energetic electrons may also be applied to positrons.

\subsubsection{The Slowing-Down Process}

Positrons emitted from typical radioisotopes have initial kinetic energies on the order of several hundred $\mathrm{keV}$ (Appendix B). When injected into a condensed medium, they gradually lose this energy through interactions with the electrons and nuclei and eventually come to a thermal equilibrium with the surrounding medium (i.e. $E_{\mathrm{p}} \approx \frac{3}{2} k_{B} T$ ). The initial positron energy is low enough that energy losses due to bremsstrahlung radiation can be ignored $^{\dagger}$. Initially, there are, therefore, only two types of physical processes to consider: inelastic collisions - the excitation and ionization of target atoms, plasmon excitations ${ }^{\ddagger}$, etc.; and elastic scattering off the target atoms. Most of the positron's initial energy is lost within a few picoseconds through inelastic collisions with the electrons. Energy losses from elastic scattering is unimportant at the initial high energies.

In metals, core ionizations, the creation of electron-hole pairs and plasmon excitations reduce $E_{\mathrm{p}}$ to a few tens of $\mathrm{eV}$ very quickly (Oliva 1980). The average energy loss per path length reaches a maximum at $E_{\mathrm{p}} \sim 20 \mathrm{eV}$ and then decreases rapidly as core ionizations and plasmon excitations become energetically impossible. Electron-hole excitation, which is allowed down to tens of $\mathrm{meV}$, also becomes less efficient with decreasing positron energy.

\footnotetext{
tDuring an inelastic collision with a nucleus, a positron can emit bremsstrahlung radiation resulting from a change in velocity due to the electrostatic repulsion of the nucleus. At very high positron energies, $E_{\mathrm{p}}>10 \mathrm{MeV}$, bremsstrahlung is the dominant energy loss mechanism. It remains efficient until about $1 \mathrm{MeV}$ and disappears below $0.5 \mathrm{MeV}$.

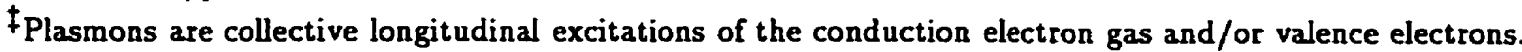




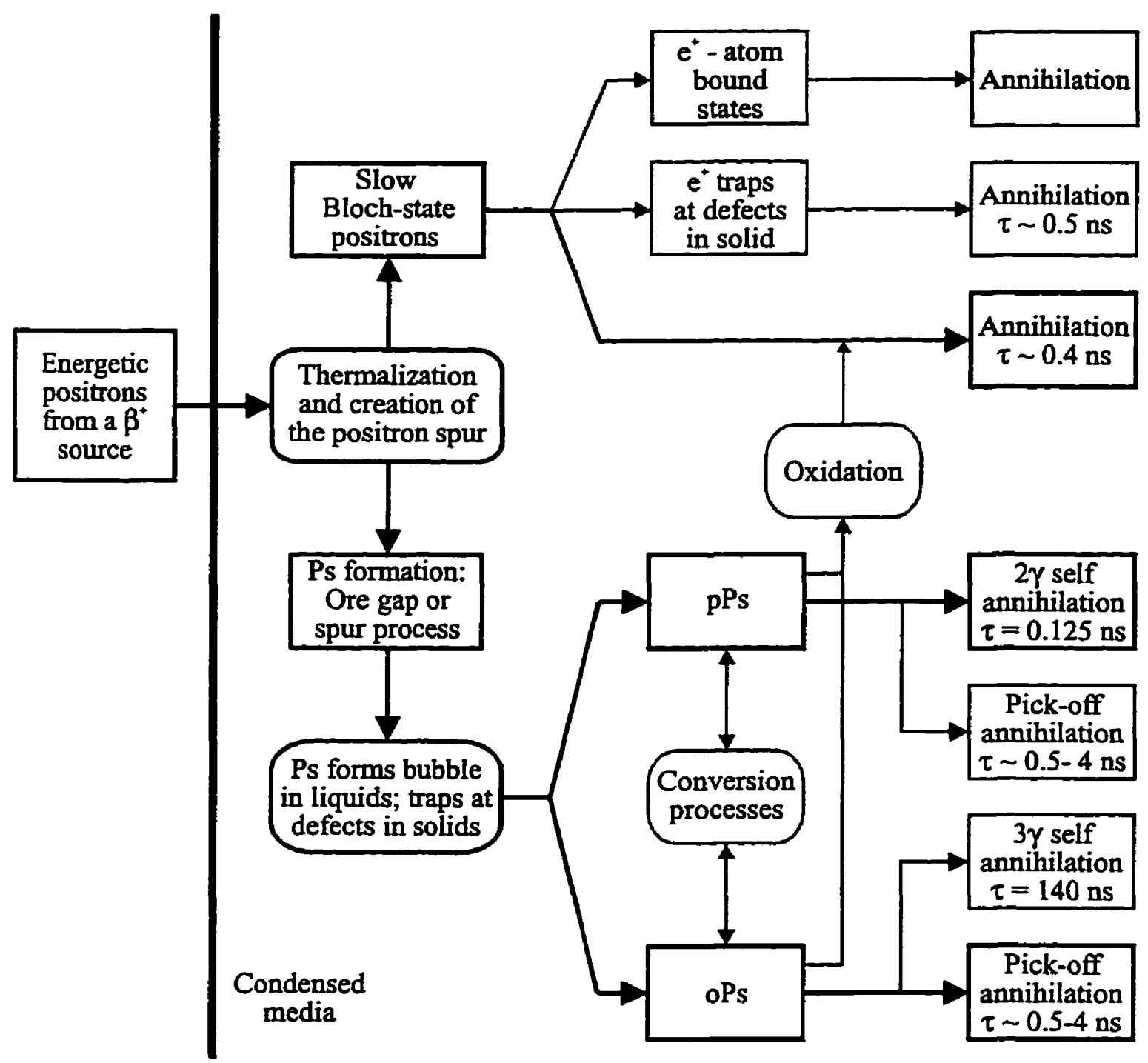

Figure 2.1: Typical positron processes in a condensed insulating material. The darker lines represent processes that are usually dominant. Energetic positrons from a radioisotope injected into a sample slow down very quickly and create an ionization trail in the process. Once thermalized, the positrons exist in a Bloch state until they eventually annihilate with an electron. Evidence of positrons trapping at large voids in rapidly condensed solid argon has been observed (Schrader et al. 1982b). It is uncertain if positrons can trap at smaller vacancies in the rare-gas solids. The possibility of positrons, and/or positronium, binding to rare-gas atoms or interacting strongly with some of the products in the ionization trail has not yet been considered in detail. In most insulating materials, some fraction of the positrons capture an electron to form positronium. Unlike positrons, thermalized positronium typically exists in a localized state - either inside a defect in rare-gas solids or inside a zero-point energy bubble in liquids (see §3.4.2). The possibility of conversions between the two different positronium spin states, para- and ortho-positronium, is discussed in §3.5.3. The positron in positronium can either self-annihilate with the electron to which it is bound or undergo pick-off annihilation with another electron in the medium. 
Below about $0.1 \mathrm{eV}$ it is replaced by inelastic phonon scattering as the dominant thermalization process (Brandt and Arista 1982; Perkins and Carbotte 1970). The average energy loss rate is a rapidly decreasing function of positron energy which means the later stages of thermalization require a longer time than the initial electronic slowing down. Nevertheless, it has long been known that positrons injected into metals thermalize in tens of picoseconds or less (Brandt and Arista 1982), which is short compared to their lifetime of about 200 ps. Therefore, only a negligible fraction of the annihilations will occur before the positron is thermalized and established in a definite, well-defined energy state. This is important, as it is usually assumed that the positron momentum is small compared to the momentum of the electron with which it annihilates - i.e. that positron annihilation can provide information about the electronic states sampled by the positron wave function.

In condensed insulating media, electronic excitations also bring the positron's energy down to a few tens of $\mathrm{eV}$ in times on the order of picoseconds (see Appendix $\mathrm{C}$ ). However, these media have a large band gap which allows the positron to fall below the threshold for inelastic scattering with a significant amount of energy remaining (i.e. $\sim 10 \mathrm{eV}$ ). In solids, the electronic excitation threshold usually lies just below the band gap energy, $E_{\text {gap }}$ (see Table A.5), due to exciton excitations ${ }^{\dagger}$ which require a minimum energy of $E_{x}=$ $E_{\mathrm{gap}}-E_{\mathrm{x}, \mathrm{b}}$ to form, where $E_{\mathrm{x}, \mathrm{b}}$ is their binding energy. In the rare-gas liquids, it is reasonable to describe these excitations in terms of either broadened atomic transitions or excitons (Schwentner et al. 1985). Positronium formation may become another important inelastic process inside a narrow range of energies, known as the 'Ore gap', around the electronic excitation threshold. Below the Ore gap Ps formation threshold, the final approach to thermal equilibrium for the monoatomic rare-gas solids is again determined by the rather inefficient phonon scattering processes. In liquids, the loss of long-range order makes it more appropriate to say that the slowing down is determined by scattering from long-wavelength density fluctuations (Basak and Cohen 1979). If the medium is very weakly interacting, as in a gas, the positron-atom interaction is better described in terms of two-particle elastic scattering. Appendix $C$ shows that most of the thermalization time is spent in this final stage of slowing down.

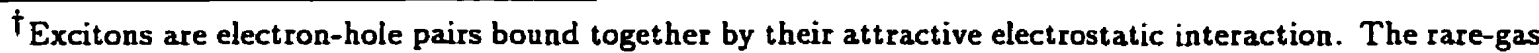
solids typically have tightly bound Frenkel excitons in which the excitation is localized on or near a single atom. A Frenkel exciton corresponds to an excited state of a single atom except that the exciton can hop between atoms by virtue of the coupling between neighbours. $E_{x, b}$ ranges from $\sim 5 \mathrm{eV}$ or less in neon to $\sim 1 \mathrm{eV}$ or less in xenon.
} 
Unlike metals, it is not at all obvious that the positron thermalization time in the condensed rare gases is short compared to the positron lifetime. The difficulty is that the final 5-10 eV of positron energy must be dissipated by phonon scattering in solids or density fluctuations in liquids. In the rare gases, the absence of optical phonon or vibrational modes reduces the efficiency of these energy loss processes even further. Gullikson and Mills (1986) have estimated that in solid argon the average positron energy loss per collision below the Ore gap Ps formation threshold is $6 \pm 2 \mathrm{meV}$ - comparable to the average acoustic phonon energy (compare this to $\hbar \omega_{p}$ in Table A.5). Thermalization times for electrons and an estimate of the positron thermalization time in Appendix $\mathrm{C}$ indicate that the positrons may not be completely thermalized prior to annihilation. Incomplete thermalization would have the effect of increasing the annihilating electron-positron pair momentum thus broadening the experimental angular correlation curves. In what follows, it is simplest to assume that the positrons are completely thermalized and then compare theory to experiment looking for the effects of non-thermalization.

Molecular media have several additional slowing-down mechanisms available which makes positron thermalization significantly faster. The lowest electronic excitations a positron can generate must have the same spin multiplicity as the ground state, since positrons can only cause spin changes through their very weak magnetic interaction. For example, a thermalizing positron can not excite an electron from the triplet $X^{3} \Sigma_{g}^{-}$ground state of $\mathrm{O}_{2}$ to either of the two lowest singlet excited states $a^{1} \Delta_{g}$ or $b^{1} \Sigma_{g}^{+}$at 0.98 and $1.63 \mathrm{eV}$, respectively. Instead, the lowest available excitation is the triplet $A^{3} \Sigma_{u}^{+}$state at $4.48 \mathrm{eV}$. Energetic electrons, on the other hand, are able to excite all of these states quite readily through their exchange interactions. Once below the Ps formation and electronic excitation thresholds, positrons may continue to lose energy by breaking bonds and by exciting intramolecular and collective vibrational and rotational modes of the molecules. The efficiency of these processes is unknown and difficult to calculate. Dissociation reactions require a considerable momentum transfer from the positron to the molecule and so are probably not very important. Intramolecular vibration excitation by a scattering electron is thought to proceed via an intermediate compound resonance state not available to the positron, so this process is probably not important as well. The average positron energy loss of $\sim 30 \mathrm{meV}$ at these low energies in solid $\mathrm{N}_{2}$ (Gullikson and Mills 1990) suggests that collective vibrational modes (optical phonons) are the most important additional thermalization mechanism available in simple condensed molecular media. 


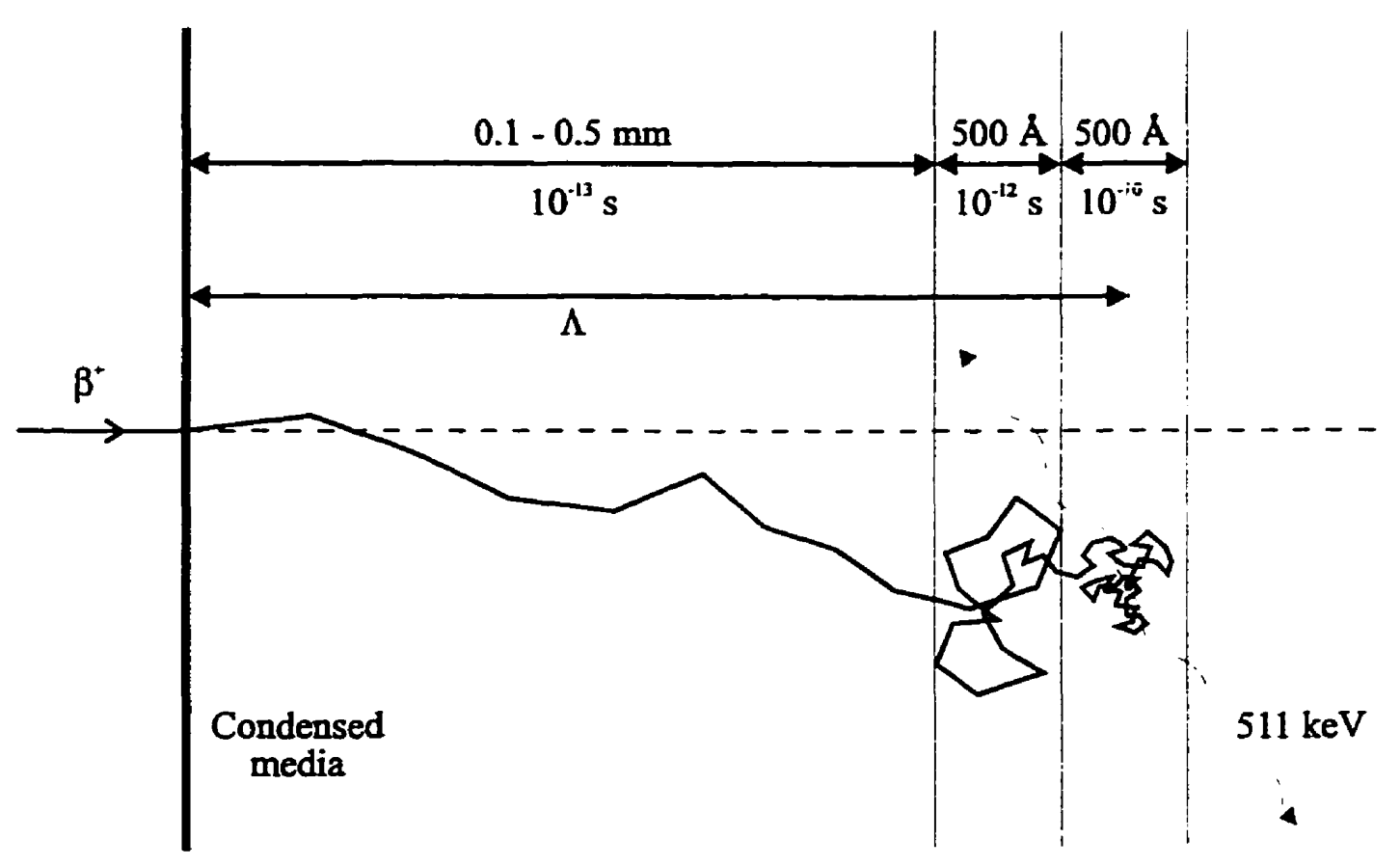

Figure 2.2: A schematic diagram of the positron ionization trail in a condensed medium. $\Lambda$ is the mean implantation depth. The final portion of the trail, known as the 'positron spur', consists of a positron, or positronium, initially surrounded by the ionization products - excited atoms, ions and secondary electrons. Once the positron's energy falls below the inelastic scattering threshold it continues to scatter off of phonons in solids or density fluctuations in liquids. A thermalized positron continues to diffuse about until it eventually annihilates with an electron, which typically produces two $511 \mathrm{keV} \gamma$-rays.

\subsubsection{The Implantation Profile}

Since the positron is a light particle, it undergoes large angular deflections during the inelastic collisions described above. However, elastic Mott scattering on target nuclei is also very important when calculating the positron's implantation profile. The reason is that positron-electron scattering is very peaked in the forward direction compared to positronnucleus scattering due to the different particle-scatterer mass ratios. Since elastic scattering has a collision frequency comparable to that for inelastic scattering, its contribution to the average angular spread in the positron trajectory cannot be ignored. The main contribution to the penetration depth occurs initially, when the high-energy positron has a large average path length between collisions with only small angular deflections, as shown in Figure 2.2. The final stages of thermalization do not increase the penetration depth significantly. Below 
$\sim 1 \mathrm{keV}$ the positron's motion becomes diffusive, with the average angular deflections becoming so large that the positron's scattering is completely isotropic. This large angle scattering simply results in a range straggling about the mean implantation depth $\Lambda$. As expected, the implantation depth increases with positron energy, while the straggling about the mean is essentially energy independent. The spatial implantation of positrons can be thought of as a spherical cloud centred at $\Lambda$ with a radius of $\sim 1000 \AA$.

The multiple scattering of positrons of initial energy $E_{\text {avg }}$ produces an implantation profile of the form

$$
I(x)=I_{0} \exp \left(-\alpha_{+} x\right)
$$

where $I(x) / I_{0}$ is the fraction of positrons left after they have passed through a distance $x$ of the material, and $\alpha_{+}=\Lambda^{-1}$ is the positron linear-absorption coefficient. For ${ }^{64} \mathrm{Cu}$ and ${ }^{68} \mathrm{Ge}$ sources, the mass absorption coefficient $\alpha_{+} / \varrho$, where $\varrho$ is the mass density, is approximately (Paulin 1981)

$$
\frac{\alpha_{+}}{\varrho}=1.1 \frac{Z^{0.25}}{E^{1.58}}\left(\mathrm{~cm}^{2} / \mathrm{g}\right)
$$

where $E=0.20 \mathrm{MeV}$ for ${ }^{64} \mathrm{Cu}$ and $0.41 \mathrm{MeV}$ for ${ }^{68} \mathrm{Ge}$. Using $(2.2)$ for ${ }^{64} \mathrm{Cu}$ the mean implantation depth for positrons in liquid $\mathrm{He}, \mathrm{Ne}, \mathrm{Ar}, \mathrm{Kr}$ and $\mathrm{Xe}$ is about 4.8, 0.33, 0.25, 0.12 and $0.09 \mathrm{~mm}$, respectively. The relatively large penetration depth in $\mathrm{He}$ is a result of its low density. These implantation depths ensure that the positrons are probing the bulk material.

Once thermalized, the positron diffuses in thermal equilibrium continuing to scatter off phonons, or density fluctuations, and impurities until it finally annihilates. As a useful first approximation, this diffusion is generally described classically in terms of an isotopic random walk process characterized by a mean free path $\lambda_{D}$ and a collision frequency $t_{D}^{-1}$ (i.e. the phonon scattering rate). The diffusion constant $D_{+}$may then be defined by the Einstein relation

$$
D_{+}=\frac{1}{3} \frac{\lambda_{D}^{2}}{t_{D}}=\frac{k_{B} T}{e} \mu_{+}
$$

where $\mu_{+}$is the positron mobility. A direct measurement of $\mu_{+}$is all but impossible in most condensed media due to experimental difficulties. Positron diffusion constants in metals and semiconductors typically range from $0.1-1.0 \mathrm{~cm}^{2} \mathrm{~s}^{-1}$ at room temperature (Nieminen 
and Manninen 1979), which implies a total average diffusion length $L$ prior to annihilation of $1000-2000 \AA$ where

$$
L=\sqrt{6 \overline{D_{+} \tau}}
$$

and $\tau \sim 200$ ps is a typical positron lifetime. Thermalized positrons therefore typically scan a volume containing $\sim 10^{9}$ atoms before annihilating. The diffusion constants in the condensed rare gases are estimated to be about an order of magnitude larger than those for metals and semiconductors. This is a direct result of the small deformation potential constant which also gives the long positron thermalization times. Assuming these estimates are reasonable, a positron in a condensed rare gas will have an average diffusion length that is an order of magnitude larger than is typical for most other materials.

\subsubsection{The Positron Spur}

High-energy positrons injected into a condensed insulating material create an ionization trail that can significantly affect the environment surrounding the thermalized positron. During the slowing-down process, the positron typically deposits tens of $\mathrm{eV}$ in the sample at each collision site. The absorption of this energy sets off a chain of events that can be grouped into three characteristic temporal stages. During the first stage, which lasts $\sim 10^{-14} \mathrm{~s}$ on the local time scale, energy is transferred from the positron to the sample with the creation of highly reactive species - excited atoms, ions, ionized electrons, etc. These 'primary' species are usually unstable and often undergo secondary reactions, either spontaneously or in collision with other atoms. During the second stage, lasting $10^{-11} \mathrm{~s}$ or so, new chemical species may be formed and in most media come to a thermal, but not chemical, equilibrium. In the final stage, which starts at about $10^{-11} \mathrm{~s}$, diffusion and reaction of the thermalized chemical species occurs.

The localized collection of species around each collision site is called a 'spur'. A typical high-energy positron produces an ionization trail containing several thousand spurs each separated by a few hundred nanometres. The size of each spur depends on the mobility of the spur products in the medium. For example, strongly scattering liquids ( $\mathrm{He}, \mathrm{Ne}, \mathrm{H}_{2}$, $\mathrm{N}_{2}, \mathrm{O}_{2}$ ) have low electron mobilities so that ionized electrons typically thermalize and diffuse about within a few nanometres of their original ions (see Table A.5). In weakly scattering liquids ( $\mathrm{Ar}, \mathrm{Kr}, \mathrm{Xe}$ ) the electrons have such high mobilities that neighbouring spurs may overlap. 
In typical radiation chemistry experiments (i.e. conductivity measurements, product analysis, etc.) the information from all the spurs is indiscriminately mixed and an average value is observed. By contrast, positron experiments rely on the annihilation event which implies that only the spurs in the last, diffusive part of the positron track can affect the observations. The terminal part of the positron track is generally referred to as the 'positron spur'. A thermalized positron finds itself close to several spurs, each initially containing one or more electron-ion pairs, excited atoms, and possibly other more complicated spur products. If the positrons, and/or any Ps that is formed, do not diffuse out of the positron spur, then they may interact with the highly reactive spur products. This could have a significant effect on the observed annihilation characteristics. The most important and perhaps interesting spur product is the ionized electrons. Given enough energy, the ionized electrons may produce more electron-ion pairs in the vicinity. These low-energy electrons, collectively named secondary electrons, diffuse about losing their energy in ways similar to that for positrons and become thermalized some distance from their original ion. Typical thermalization times and distances for secondary electrons are given in Table C.1. Many of the ionized electrons recombine with their ion due to the Coulombic attraction producing an excited state (geminate neutralization), while others combine with one of the other available ions (random neutralization). Electrons that escape the ionic attractions remain free to perhaps combine with a positron to form positronium or to interact with other species and/or positrons and positronium. It is these sorts of possibilities that will make the positron spur an important consideration.

\subsection{Positron States in Condensed Matter}

A thermalized positron in condensed matter basically has three distinct states from which it can annihilate, as shown in Figure 2.1. These are a delocalized Bloch state in the bulk, a bound state in a positron-molecule complex, and in solids, a localized state trapped at a defect. In most condensed materials, the thermalized positrons typically remain 'free' in their lowest energy Bloch state near the bottom of their 1s band - until they eventually annihilate. Since positrons are strongly repelled from the positively charged nuclei they have a small wave-function amplitude around each atomic core which increases rapidly to become largest in the interstitial regions. Bloch state positrons are said to have a 'Swiss cheese' like spatial distribution with a reasonably constant density throughout the medium except for 
'holes' around each atom due to the strong repulsion. The proper interpretation of positron experiments requires a knowledge of the overlap between the electron and the positron wave functions in the medium. The techniques used to calculate these wave functions and their corresponding band energies will be discussed in §2.4.1.

Positron annihilation studies have made substantial contributions to the study of lattice defects in solids. The Coulomb repulsion felt by the positron causes it to see any region of lower than average positive charge as an attractive potential well. If the defects provide a well that is deep and/or large enough, it is possible for positrons to trap there in localized states at a characteristic rate. The delocalized and localized states are usually distinguished by their annihilation characteristics. The possibility of positron trapping at defects in the rare-gas solids is discussed in $\$ 2.4 .2$. The possibility of localized selftrapped positron states (i.e. ones where the positron causes the structural rearrangements) in condensed matter will also be mentioned.

Figure 2.1 suggests that positrons may form bound states with the atoms, or molecules, of the sample. To date, there is no experimental evidence to suggest that positrons form bound states in any of the media studied in this thesis. Several theoretical models suggest that positrons cannot bind to rare gas atoms (Golden and Epstein 1974; Schrader 1979; Karl et al. 1984) or to the diatomic molecules $\mathrm{H}_{2}, \mathrm{~N}_{2}$ or $\mathrm{O}_{2}$ (Schrader and Wang 1976). However, while the heavier rare gases and $\mathrm{H}_{2}$ are calculated to have negative positron affinities, implying no bound state, the affinity is small enough that binding cannot be definitively ruled out. Furthermore, the changes to the affinities that occur when the atoms are brought together to form a condensed medium remain unclear. Despite this, the models and results presented in this and the following chapters make positron binding to atoms or molecules in the condensed rare or diatomic gases an unlikely possibility.

\subsubsection{Delocalized Positron Wave Functions}

The annihilation characteristics of free positrons are determined by the overlap of their Bloch state wave function with the electron wave functions of the medium. In perfect (and infinite) crystalline solids, the crystal potential $V(\mathbf{r})$ is periodic, which means the positron interaction with the lattice is also periodic and invariant under translation by a lattice vector. The total crystal momentum $\hbar k$ is a good quantum number to label the 
eigenstates $\Psi_{k}$ and the corresponding eigenenergies $E_{k}$. The Bloch state wave function can therefore be written as

$$
\Psi_{\mathbf{k}}(\mathbf{r})=\exp (\mathbf{i k} \cdot \mathbf{r}) u_{\mathbf{k}}(\mathbf{r})
$$

where $u_{\mathbf{k}}(\mathbf{r})$ has the periodicity of the lattice. These wave functions are usually obtained from band structure calculations, the object of which is to solve the Schrödinger equation for a given $V(\mathbf{r})$

$$
-\frac{\hbar^{2}}{2 m} \nabla^{2} \Psi_{\mathbf{k}}(\mathbf{r})+V(\mathbf{r}) \Psi_{\mathbf{k}}(r)=E_{\mathbf{k}} \Psi_{\mathbf{k}}(\mathbf{r})
$$

The various techniques used to solve (2.6) differ in the form chosen for $\Psi_{k}(\mathbf{r})$. The techniques used in this work to calculate solid-state positron energies and wave functions include the simple Wigner-Seitz method and the more sophisticated augmented-plane-wave (APW) and pseudopotential methods. The tight-binding nature of the insulators studied in this work, make it reasonable to approximate the solid-state electron wave functions using atomic wave functions, for which tabulations and analytic forms exist. Actual solid-state electron wave functions could also be calculated using the APW method. In general, the loss of long-range order in liquids makes them difficult to treat using solid-state techniques. The rare-gas liquids, however, exist over a small temperature range and have many properties that are similar to those of the solid. Treating the liquid as if it were a solid is expected to be a reasonable approach so long as the long-range disorder does not play an important role in determining the annihilation characteristics. Since these characteristics depend mainly on the overlap between the positron and the electron wave functions around each atomic site this is expected to be the case. In fact, even low-energy positron-rare-gas atom scattering calculations are able to provide a reasonable first approximation to the annihilating electronpositron momentum distribution in the condensed rare gases, as will be seen in \$5.1.1. The following presents a brief description of the methods used to calculate the required positron wave functions and energies.

\subsubsection{The Wigner-Seitz Approximation}

The first serious attempt to calculate a positron wave function was undertaken by Berko and Plaskett (1958) who made use of the Wigner-Seitz approximation. A thermalized Bloch-state positron will, by definition, have a wave function that is the same within each 
Wigner-Seitz cell. To calculate the wave function inside a polyhedral cell with its complicated boundary conditions is a difficult problem. The approximation that is often made is to replace the cell with an equal volume sphere of radius $R_{w s}$ inside of which the potential $V(\mathbf{r})$ is constructed to be spherically symmetric. The Wigner-Seitz energy, $E_{w s}$, is usually taken to be the lowest energy for which the $1 \mathrm{~s}(\mathbf{k}=0)$ positron wave function satisfies the radial Schrödinger equation

$$
-\frac{\hbar^{2}}{2 m} \frac{d^{2} P_{0}(\rho)}{d \rho^{2}}+V(\rho) P_{0}(\rho)=E_{\mathrm{ws}} P_{0}(\rho), \quad \rho \leq R_{\mathrm{ws}}
$$

where $\rho=\mathbf{r}-\mathbf{R}_{\nu}$ is measured from the centre of the $\nu^{\text {th }}$ Wigner-Seitz sphere. The positron wave function $\Psi_{0}(\rho)=P_{0}(\rho) / \rho$ is also spherically symmetric and satisfies the boundary condition $d \Psi_{0}(\rho) / d \rho=0$ at $\rho=R_{\text {ws }}$. A typical Wigner-Seitz wave function for positrons in solid argon, calculated using Numerov integration techniques (Chow 1972), is shown in Figure 5.6. The wave function closely approaches zero near each atomic core, though for light atoms the amplitude $\Psi_{0}(0)$ may be significant. The amplitude at small $\rho$ will determine the annihilation rate with the tightly bound core electrons. While the Wigner-Seitz wave function is accurate near each core, its spherical symmetry does not reflect any of the angularity of the crystal in the interstitial regions. The result depends only on the atomic volume, not on the the actual geometry of the lattice - i.e. a liquid at the same density would give a similar result. The part of the wave function in the interstitial region may however be very important as the positron spends most of its time in these regions. Methods for extending the calculation of positron wave functions into the interstitial regions have since been devised. One particularly relevant attempt was that of Rose and DeBenedetti (1965) who used a 'muffin-tin' extension to the Wigner-Seitz model to calculate the electronpositron momentum distribution in solid argon. While their results were more realistic physically, their approach required the evaluation of awkward two-centre integrals.

\subsubsection{The Augmented Plane Wave Method}

One simple-minded way to overcome the limitations of the Wigner-Seitz approach is to expand $\Psi_{k}(\mathbf{r})$ into an infinite set of plane waves

$$
\Psi_{\mathbf{k}}(\mathbf{r})=\Omega^{-1 / 2} \sum_{j} A_{j}(\mathbf{k}) \exp \left(\mathrm{i} \mathrm{k}_{j} \cdot \mathbf{r}\right)
$$

where $\mathbf{k}_{j}=\mathbf{k}+\mathbf{G}_{j}, \mathbf{G}_{j}$ are reciprocal lattice vectors and $\Omega=N \Omega_{0}$ is the crystal volume with unit cell volume $\Omega_{o}$. Equation $(2.8)$ has the required Bloch form. Inserting this 
into the Schrödinger equation results in a set of simultaneous equations in the momentum representation with the plane wave coefficients $A_{j}(\mathbf{k})$ as unknowns

$$
\left(\frac{\hbar^{2}}{2 m} \mathbf{k}_{j}^{2}-E\right) A_{j}(\mathbf{k})+\sum_{i} V_{i j} A_{i}(\mathbf{k})=0 \text {. }
$$

The matrix elements $V_{i j}$ may be deduced from X-ray scattering data (Stroud and Ehrenreich 1968) or from the Fourier transform of the potential (Gould et al. 1972)

$$
V_{i j}=S\left(\mathbf{k}_{i j}\right) \int d \mathbf{r} v(\mathbf{r}) e^{i \mathbf{k}_{i,} \cdot \mathbf{r}}
$$

where $\mathbf{k}_{i j}=\mathbf{k}_{i}-\mathbf{k}_{j}=\mathbf{G}_{i}-\mathbf{G}_{j}$. Information about the atomic positions is contained in the structure factor

$$
S(\mathbf{q})=\frac{1}{N} \sum_{\nu} e^{\mathrm{i} \mathbf{q} \cdot \mathbf{R}_{\nu}}
$$

The $V_{i j}$ are large enough that low-order perturbation theory cannot be used to solve the equations. Instead (2.9) must be solved using a limited number of plane waves and finding those values of $E$ for which the secular determinant of (2.9) has nontrivial solutions. Solutions for different $\mathbf{k}$ gives the positron band structure $E_{\mathbf{k}}$ and the plane wave coefficients. This technique has had some success for positrons in crystals. However, it requires a rather large number of plane waves to obtain convergence and does not accurately represent the wave function near the atomic cores. The expansion of electron wave functions in terms of plane waves converges much more slowly due to the rapid oscillations near each of the nuclei, resulting from orthogonalization to the core-electron wave functions, which require high-momentum components.

One way to improve the rate of convergence is to modify the plane-wave expansion and include a certain amount of high-momentum components from the outset. This is the approach used in both the orthogonalized-plane-wave and augmented-plane-wave methods. Both methods are widely used in the computation of electronic band structures. In this work, the APW method is used to calculate the positron band structure and wave functions which, in turn, are used to calculate the positron's annihilation characteristics. The APW method has been well described elsewhere so only the essentials are presented here. One particularly useful description is that of Loucks (1967) which includes listings of the necessary computer code.

In the APW method the wave functions are expanded in terms of 'augmented' plane 
waves $\chi_{\mathbf{k}_{1}}$ such that

$$
\Psi_{\mathbf{k}}(\mathbf{r})=\Omega^{-1 / 2} \sum_{j} A_{j}(\mathbf{k}) \chi_{\mathbf{k},}(\mathbf{r})
$$

The APW method requires $V(\mathbf{r})$ to have a 'muffin-tin' form - i.e. it is spherically symmetric within non-overlapping spheres of radius $R_{\mathrm{MT}}$, centred at the atomic positions $R_{\nu}$, and constant in the interstitial regions so that

$$
V(\mathbf{r})=\sum_{\nu} v\left(\mathbf{r}-\mathbf{R}_{\nu}\right)
$$

where

$$
v(\mathbf{r})= \begin{cases}v(r) & r<R_{\mathrm{MT}} \\ v_{0} & r>R_{\mathrm{MT}}\end{cases}
$$

The construction of the 'muffin-tin' potentials used in this work is described in Appendix H. Since the potential between the spheres is constant, the APW functions in this region are simply plane waves (i.e. $\chi_{\mathbf{k}},(\mathbf{r})=\exp \left(i \mathbf{k}_{j} \cdot \mathbf{r}\right)$ ). The solutions to the Schrödinger equation inside each muffin-tin sphere have the form

$$
\phi_{n ! m}(\boldsymbol{\rho})=U_{n l}(\rho) Y_{l m}(\hat{\rho})
$$

where the $U_{n l}(\rho)=P_{n l}(\rho) / \rho$ satisfy the radial Schrödinger equation, and the $Y_{l m}(\hat{\rho})$ are the usual spherical harmonics. The APW function inside each sphere is taken to be a linear combination of these solutions

$$
\chi(\rho)=\sum_{l=0}^{\infty} \sum_{m=-l}^{+l} C_{l m} U_{l}(\rho, E) Y_{l m}(\hat{\rho})
$$

where the energy dependence has been included in the radial wave functions. The coefficients $C_{l m}$ are chosen so that $\chi(\rho)$ exactly matches the exterior plane waves at the sphere surfaces $\rho=R_{\mathrm{Mr}}$. When this is done (2.15) inside the $\nu^{\text {th }}$ sphere becomes

$$
\chi_{\mathbf{k},}(\mathbf{r})=4 \pi e^{i \mathbf{k}_{3} \cdot \mathbf{R}_{\nu}} \sum_{l=0}^{\infty} \sum_{m=-l}^{+l} \mathrm{i}^{l} J_{l}\left(k R_{\mathrm{MT}}\right) \frac{U_{l}(\rho, E)}{U_{l}\left(R_{\mathrm{MT}}, E\right)} Y_{l m}^{*}\left(\dot{k}_{j}\right) Y_{l \mathrm{~m}}(\hat{\rho})
$$

where $J_{l}$ is a spherical Bessel function of order $l$ and where only one atom per unit cell has been assumed.

The next step is to insert $\chi_{k_{j}}(\mathbf{r})$ into (2.12) and use this as a trial function for a variational estimate of the energy. The coefficients $A_{j}(\mathrm{k})$ in $(2.12)$ satisfy equations like those of (2.10) except that the Fourier components of the potential are replaced by a 
more complicated expression $\left(V_{i j} \rightarrow \Gamma_{i j}\right)$ which is an integral involving $\chi_{\mathbf{k}_{\mathbf{i}}}, \chi_{\mathbf{k}}$, and the Hamiltonian operator associated with the periodic potential. The matrix elements are now given by

$$
\begin{aligned}
\Gamma_{i j}=-4 \pi R_{\mathrm{MT}}^{2} & \left(\left(\mathbf{k}_{i} \cdot \mathbf{k}_{j}-E\right) \frac{J_{1}\left(k_{i j} R_{\mathrm{MT}}\right)}{k_{i j}}\right. \\
& \left.-\sum_{l=0}^{\infty}(2 l+1) P_{l}\left(\bar{k}_{i} \cdot \dot{k}_{j}\right) J_{l}\left(k_{i} R_{\mathrm{MT}}\right) J_{l}\left(k_{j} R_{\mathrm{MT}}\right) \frac{U_{l}^{\prime}\left(R_{\mathrm{MT}}, E\right)}{U_{l}\left(R_{\mathrm{MT}}, E\right)}\right)
\end{aligned}
$$

where $P_{l}$ is the Legendre polynomial of order $l$ and $U_{l}^{\prime} / U_{l}$ is the logarithmic derivative of the radial wave function at the muffin-tin radius. The band structure problem is solved by seeking a solution of (2.9) with $\Gamma_{i j}$, which exists when the secular determinant is zero. For a given value of $\mathbf{k}$, one can calculate the determinant for a given value of $E$, which can then be varied until a root is found. This value of $E$ is then the estimate of $E_{\mathbf{k}}$. The corresponding plane-wave coefficients can be used in $(2.12)$ to calculate the wave function.

\subsubsection{The Pseudopotential Method}

One particularly convenient technique for calculating positron energies and wave functions is the 'pseudopotential' method (Stott and Kubica 1975; Kubica 1975a; Kubica et al. 1975b; Stott and West 1978). This method combines the proper behaviour near the atomic cores given by the Wigner-Seitz method with the anisotropy given by the crystal lattice and has the advantage that it can be solved using low-order perturbation theory. It also provides a simple means of calculating the low-energy deformation potential parameter (Appendix $\mathrm{C}$ ), the positron band effective mass and the positron energy in insulating liquids.

In this approach, the positron wave function for states near the bottom of the lowest energy band, $\Psi_{k}(r)$, is factored into two parts

$$
\Psi_{\mathbf{k}}(\mathbf{r})=U(\mathbf{r}) \psi_{\mathbf{k}}(\mathbf{r})
$$

$U(\mathbf{r})$ contains the effect of the strong repulsion of the positron from the atomic cores and is insersitive to the positron energy or any angularity within the lattice. $\psi_{\mathbf{k}}(\mathbf{r})$ is a smooth envelope pseudo wave function which contains all the angular and energy dependence of the total wave function. The justification for this separation lies in the fact that the positron wave function is most distorted in the core regions where the potential is much larger than the positron energies of interest. This means that the shape of $\Psi_{k}(r)$ must be insensitive to 
the positron energy in the core region and so an energy independent term can be factored out. The core wave function $U(r)$ is taken to satisfy (2.7) using a spherically symmetric single atom potential $V_{\text {at }}(r)$ with the Wigner-Seitz boundary condition imposed at a conveniently chosen 'muffin-tin' radius. In solids, $\boldsymbol{R}_{\mathrm{MT}}$ usually corresponds to the largest sphere that can be inscribed inside the Wigner-Seitz cell. For liquids, the sphere radius is chosen to be $R_{M \tau}=\left(3 /\left(4 \pi n_{o}\right)\right)^{1 / 3}$ where $n_{o}$ is the average number density. Between the muffin-tin spheres the core factor is assumed to be constant, $U(r)=U\left(R_{\mathrm{MT}}\right)$ (typically $U\left(R_{\mathrm{MT}}\right)=1$ ).

Substituting (2.18) into the full Schrödinger equation gives a similar equation for the pseudo wave function $\psi_{\mathbf{k}}(\mathbf{r})$

$$
\left(-\frac{\hbar^{2}}{2 m} \nabla^{2}+W(\mathbf{r})\right) \psi_{\mathbf{k}}(\mathbf{r})=E_{\mathbf{k}} \psi_{\mathbf{k}}(\mathbf{r})
$$

where the pseudopotential $W(\mathbf{r})$ within the $\nu^{\text {th }}$ muffin tin is

$$
W(\mathbf{r})=E_{\mathrm{ws}}+V(\mathbf{r})-V_{\mathrm{at}}\left(\mathbf{r}-\mathbf{R}_{\nu}\right)-\frac{\hbar^{2}}{m} \frac{\nabla U(\mathbf{r})}{U(\mathbf{r})} \dot{r} \cdot \nabla
$$

while between the muffin tins $W(\mathbf{r})=V(\mathbf{r})$. The gradient part of $W(\mathbf{r})$ is generally small so that within each muffin-tin sphere the pseudopotential is quite weak due to the near cancellation of the $V(\mathbf{r})$ and $V_{\mathrm{at}}(\mathbf{r})$ terms. The pseudo wave function may now be solved in a number of ways. As described above, it can be expanded in a limited set of plane waves giving a secular equation which can be solved to give the plane-wave coefficients and the positron energy. However, since $W(\mathbf{r})$ is small it is more convenient to treat it as a perturbation to free particle-like behaviour (with $E_{\mathbf{k}}=E_{\mathrm{ws}}+\hbar^{2} k^{2} /(2 m)$ ). An accurate solution to (2.19) can be obtained from second-order perturbation theory. In the plane wave representation

$$
\langle\mathbf{p}|W| \mathbf{k}\rangle=\frac{S^{*}(\mathbf{q})}{\Omega_{o}}(w(q)+\mathbf{k} \cdot \mathbf{q} B(q))
$$

where $\mathbf{q}=\mathbf{k}-\mathbf{p}$, the structure factor is given by (2.11), and the single-centre parts are

$$
\begin{aligned}
& \omega(q)=4 \pi R_{\mathrm{MT}}^{3} E_{\mathrm{ws}} \frac{j_{1}\left(q R_{\mathrm{MT}}\right)}{q R_{\mathrm{MT}}}+\int d \mathbf{r} V(\mathbf{r}) e^{\mathrm{iq} \cdot \mathrm{r}}-4 \pi \int_{0}^{R_{\mathrm{MT}}} d r r^{2} V_{\mathrm{at}}(r) j_{0}(q r) \\
& B(q)=-4 \pi \int_{0}^{R_{\mathrm{MTT}}} d r r^{2} \ln (U(r)) j_{0}(q r) .
\end{aligned}
$$

where $j_{0}$ and $j_{1}$ are spherical Bessel functions and $U\left(R_{\mathrm{MT}}\right)$ has arbitrarily been set to 1 . 
Using (2.21)

$$
\begin{aligned}
\psi_{\mathbf{k}}(\mathbf{r}) & =\frac{C_{\mathbf{k}}}{\sqrt{\Omega}}\left(|\mathbf{k}\rangle+\frac{2 m}{\hbar^{2}} \sum_{\mathbf{p} \neq \mathbf{k}} \frac{\langle\mathbf{p}|W| \mathbf{k}\rangle}{k^{2}-p^{2}}|\mathbf{p}\rangle\right) \\
& =\frac{C_{\mathbf{k}}}{\sqrt{\Omega}}\left(e^{i \mathbf{k} \cdot \mathbf{r}}-\frac{2 m}{\hbar^{2} \Omega} \sum_{\mathbf{p} \neq \mathbf{k}} S^{*}(\mathbf{q}) \frac{(\omega(q)+\mathbf{k} \cdot \mathbf{q} B(q))}{p^{2}-k^{2}} e^{i \mathbf{p} \cdot \mathbf{r}}\right)
\end{aligned}
$$

where $C_{k}$ ensures normalization of the full positron wave function and

$$
\begin{aligned}
E_{\mathbf{k}} & =\frac{\hbar^{2} k^{2}}{2 m}+\langle\mathbf{k}|W| \mathbf{k}\rangle+\frac{2 m}{\hbar^{2}} \sum_{\mathbf{p} \neq \mathbf{k}}|S(\mathbf{q})|^{2} \frac{\langle\mathbf{p}|W| \mathbf{k}\rangle\langle\mathbf{k}|W| \mathbf{p}\rangle}{k^{2}-p^{2}} \\
& =\frac{\hbar^{2} k^{2}}{2 m}+\frac{\omega(0)}{\Omega_{0}}-\frac{2 m}{\hbar^{2} \Omega_{0}^{2}} \sum_{\mathbf{p} \neq \mathbf{k}}|S(\mathbf{q})|^{2} \frac{(\omega(q)+\mathbf{k} \cdot \mathbf{q} B(q))(\omega(q)-\mathbf{p} \cdot \mathbf{q} B(q))}{p^{2}-k^{2}} .
\end{aligned}
$$

To first order the normalization constant is

$$
\left|C_{\mathbf{k}}\right|^{2}=\frac{\Omega_{o}}{\Omega_{o}-\chi(0)}\left(1-\frac{4 m}{\hbar^{2} \Omega_{o}} \sum_{\mathbf{p} \neq \mathbf{k}} \frac{\omega(q)+\mathbf{k} \cdot \mathbf{q} B(q)}{p^{2}-k^{2}}\right)\left(\frac{\chi(q)}{\Omega_{o}-\chi(0)}|S(q)|^{2}\right)
$$

with

$$
\chi(q)=4 \pi \int_{0}^{R_{\mathrm{MT}}} d r r^{2}\left(1-U^{2}(r)\right) j_{0}(q r)
$$

For a thermalized positron near the bottom of its lowest energy level $(\mathbf{k} \sim 0)$ the above equations reduce to

$$
\begin{aligned}
\psi_{0}(\mathbf{r}) & =\frac{C_{0}}{\sqrt{\Omega}}\left(1-\frac{2 m}{\hbar^{2} \Omega_{o}} \sum_{\mathbf{p} \neq 0} S^{*}(\mathbf{p}) \frac{\omega(p)}{p^{2}} e^{i \mathbf{p} \cdot \mathbf{r}}\right) \\
E_{o} & =\frac{3}{2} k_{B} T+\frac{\omega(0)}{\Omega_{o}}-\frac{2 m}{\hbar^{2} \Omega_{o}^{2}} \sum_{\mathbf{p} \neq 0}|S(\mathbf{p})|^{2} \frac{\omega(p)\left(\omega(p)+p^{2} B(p)\right)}{p^{2}} .
\end{aligned}
$$

If the lattice is assumed rigid then $S(\mathbf{q})=\delta_{\mathbf{q}, \mathbf{G}_{\nu}}$. The pseudopotential formalism has also been used to study the effects of lattice vibrations on the positron distribution (Stott and West 1978).

Nieminen et al. (1980) obtained a simple equation for the energy of a thermalized positron in insulating liquids by averaging over the atomic sites (i.e. $\sum_{p} \rightarrow \Omega /(2 \pi)^{3} \int d \mathbf{p}$ )

$$
E_{0}=\frac{3}{2} k_{B} T+\frac{\omega(0)}{\Omega_{0}}-\frac{m}{\hbar^{2} \pi^{2} \Omega_{0}} \int_{0}^{\infty} d p S_{L}(p) \omega(p)\left(\omega(p)+p^{2} B(p)\right)
$$


$S_{L}(\mathbf{p})$ is the liquid structure factor defined as

$$
S_{L}(\mathbf{p})=\frac{1}{N} \sum_{\nu} \sum_{\nu^{\prime}} e^{\mathrm{i} \mathbf{p} \cdot\left(\mathbf{R}_{\nu^{\prime}}-\mathbf{R}_{\nu}\right)}
$$

and is the quantity measured in neutron scattering experiments.

The calculational details and results obtained from the APW and pseudopotential methods are discussed in $§ 5.3$.

\subsubsection{Localized Positron States}

For over 25 years, positron annihilation techniques have been used to study defects in a wide variety of solids. Positrons have good sensitivity to defects due to the Coulomb repulsion it feels from the ionic cores which causes it to see hole-type defects as attractive potential wells. If these wells are large enough, it is possible for the positron to trap there from the delocalized state at a characteristic rate. A typical trapping rate per unit defect concentration is $\sim 10^{15} \mathrm{~s}^{-1}$ which implies that defect trapping becomes significant and competes with annihilation for defect concentrations greater than $\sim 10^{-5}$ defects per atom. At high enough concentrations, the fraction of positrons annihilating from defects may be comparable to, or even greater than, that annihilating from delocalized states. Since a positron in a defect is in a very different environment, its annihilation characteristics can usually be distinguished from those of the delocalized states. The trapped positron sees an overall reduction in the electron density so it is expected that its lifetime will be greater than that for a delocalized positron. Furthermore, its overlap with the innermost core electrons is significantly reduced which results in a relative increase in the low momentum contributions to its momentum density.

Unlike metals, is very difficult to produce rare-gas solid samples that are defect-free single crystals. Under the growing conditions used in this work, the samples were polycrystalline and likely contained a reasonable number of defects (Venables and Smith 1977). To date, positron binding energies to various kinds of defects in the rare-gas solids have neither been measured nor calculated theoretically. It is therefore uncertain whether or not it is energetically favourable for positrons to trap at the defects typically found in these solids. Haberl (1979a,1979b) performed lifetime experiments in well annealed samples of solid neon and argon over a wide temperature range. At low temperatures, well annealed samples should have few vacancies and the positrons remain largely delocalized. As the temperature 
increases the positron lifetime should also increase slowly due to thermal expansion of the lattice, which reduces the electron-positron overlap. The thermally generated vacancy concentration also increases with temperature so if positrons do trap at vacancies then at some point their lifetime should show a steeper rise with temperature than can be explained by thermal expansion. The main conclusion of Haberl's work was that increases in positron lifetime could be accounted for by thermal expansion - the results showed no evidence of the characteristic curve for thermal vacancy trapping. The implication is that positrons do not trap at monovacancies, which are the dominant thermally generated defects in the rare-gas solids.

The only other relevant experimental data is that of Schrader et al. (1982) who prepared solid argon samples by rapidly condensing argon onto a cold surface producing highly defective lattices. Using isochronal annealing techniques, a series of lifetime experiments were performed on samples containing different concentrations and sizes of large void defects. The results showed a remarkable correspondence between the lifetimes and intensities of the positron and Ps components as a function of annealing temperature. A reasonable explanation for these results was the early trapping of positrons into the large voids followed by the rapid reaction with spur electrons which resulted in either annihilation or Ps formation. However, while this work implies that positrons do trap at large voids, the concentration and size of the defects are likely somewhat larger than those existing in typical solid samples. Perhaps the most convincing evidence against positrons trapping at typical defects in the rare-gas solids is that the positron component of the momentum density is essentially identical to that in liquids. Since positrons remain delocalized in the rare-gas liquids (i.e. their thermal wavelength is much greater than the size of the shortlived density fluctuations) the implication is that positrons also annihilate from delocalized states in the rare-gas solids.

The above discussion was concerned with positrons binding to pre-existing defects in solids. It is also interesting to consider the possibility of a positron creating a structural deformation and then becoming localized there, in what are known as self-trapped states. In solids, there are two main types of self-trapping to consider: 'extended' self-trapping in which the positron retains much of its Bloch state character but becomes trapped by a weakly distorted lattice; and 'compact' self-trapping in which the positron actually creates a lattice defect. Leung et al. (1976) have considered the possibility of extended positron self-trapping in metals by considering the positron-phonon coupling in the long-wavelength 
limit. From their simple model, they concluded that extended self-trapping was unlikely to occur in metals because the coupling of the positron to the lattice was too small. Appendix D shows that it is also unlikely to occur in the condensed rare gases. In some solids, compact self-trapping may be energetically favourable, if the defect formation energy is less than positron binding energy to the defect. However, there is a difficulty in that the structural rearrangements needed for compact self-trapping typically require times that are considerably longer than the positron lifetime. To date, positron compact self-trapping has never been observed.

\subsection{Positron Annihilation}

In a vacuum, the positron is a stable particle with a lifetime greater than $10^{22}$ years (Bellotti et al. 1983). However, once injected into a gas or condensed matter, the positron is certain to annihilate with an electron. This annihilation is a relativistic process in which the particle masses are converted into $\gamma$-rays. The process is considered to be well understood on the basis of quantum electrodynamics. Detailed discussions of the selection rules and other considerations that govern the decay can be found in many fundamental texts (Jauch and Rohrlich 1980; Berestetskii et al. 1982).

Information about positron and positronium interactions in a medium must be inferred from the experimental observation of the $\gamma$-rays resulting from the annihilation. An important advantage of this method is that the high-energy $\gamma$-rays leave the medium relatively unscattered and unattenuated carrying away information about the positron and positronium lifetimes, and the energy and momentum distribution of the annihilated electron-positron pairs. A disadvantage is that the positron distorts the very electronic structure it is being used to probe. The following gives a description of the annihilation event which ignores the electron-positron correlation effects in order to make the basic properties of the process clear. This is followed by a description of the annihilation process in condensed matter which includes a brief discussion of the relevant approaches used to cope with the many-body correlations. 


\subsubsection{The Annihilation Event}

Positron annihilation is a relativistic process described by quantum electrodynamics. The annihilation process provides an energy of $\sim 2 m_{0} c^{2}$ - the total rest mass energy of the electron-positron pair. Conservation of momentum requires that at least two other bodies (particles and/or photons) be involved in the process. In principle any number of photons may be produced during the annihilation process. However, there are several dominant factors that determine the probability of the various modes of electron-positron annihilation. First, there is a reluctance to transfer momentum between particles of very different masses - the cross-section for a process in which such a transfer occurs is reduced by a factor on the order of $\alpha^{3}$, where $\alpha=1 / 137.036$ is the fine structure constant. Secondly, the cross-section for annihilation into $(n+1)$ photons is a factor of approximately $\alpha$ smaller than for annihilations into $n$ photons. Finally, there is the requirement of $C P$ invariance for electromagnetic interactions, which is equivalent to the conservation of charge parity for the electron-positron pair.

Zero-photon annihilation requires two other particles, such as nucleons in the same nucleus, be involved to conserve momentum and energy. This makes the process highly unlikely due to the unfavourable momentum transfer required. Single-photon annihilation requires the presence of only one other particle to absorb the recoil momentum. The crosssection for the single-photon annihilation of a slow, free positron with a 1s electron of an atom of atomic number $Z$, and with the nucleus absorbing the recoil momentum is (Fermi and Uhlenbeck 1933)

$$
\bar{\sigma}_{1 \gamma}=\frac{4}{3} \pi r_{0}^{2} Z^{5} \alpha^{4} \frac{v}{c}
$$

where $r_{o}=\alpha^{2} a_{o}$ is the classical electron radius, $a_{o}$ is the Bohr radius, and $v$ is the positron velocity (the electron is assumed to be at rest). Annihilation into two or more photons does not require the presence of another particle - all the conservation laws can be satisfied by the photons themselves. From the uncertainty principle one can deduce that for a $2 \gamma$ annihilation the electron-positron pair must be within $\sim 10^{-13} \mathrm{~m}$ of each other, which amounts to contact on the atomic scale. The short range of the annihilation interaction implies that slow electron-positron pairs will annihilate at a reasonable rate only if they are in a relative s-state - the electron-positron wave function overlap is negligible for higher orbitals. A slow electron-positron pair must therefore annihilate from either a spin singlet $\left({ }^{1} S_{0}, S=0\right)$ or a spin triplet state $\left({ }^{3} S_{1}, S=1\right)$. The conservation of charge parity (see 
Appendix B) requires that the singlet state annihilate into an even number of photons while the triplet state must decay into an odd number of photons. The cross-section for $2 \gamma$ annihilation of a slow, free positron with a free electron, averaged over all spin-directions and photon polarizations and ignoring the Coulomb attraction, is (Dirac 1930)

$$
\bar{\sigma}_{2 \gamma}=\pi r_{a}^{2} \frac{c}{v}
$$

The equivalent spin-averaged $3 \gamma$ annihilation cross-section is simply a constant times the $2 \gamma$ cross-section (Ore and Powell 1949)

$$
\bar{\sigma}_{3 \gamma} \approx \epsilon \bar{\sigma}_{2 \gamma}, \text { where } \epsilon \approx \frac{4}{3 \pi}\left(\pi^{2}-9\right) \alpha=\frac{1}{371.3} .
$$

When small radiative corrections are accounted for $\epsilon=1 / 378.16$. Since the annihilation cross-sections decrease rapidly with the number of emitted photons only 1,2 and 3 photon annihilation need be considered in practice. However, at low positron velocities, $\bar{\sigma}_{1 \gamma}$ becomes negligible compared to $\bar{\sigma}_{2 \gamma}$ and $\bar{\sigma}_{3 \gamma}$. At energies of $100 \mathrm{eV}$ even for the heaviest atoms (i.e. lead $Z=82$ ) the ratio $\bar{\sigma}_{1 \gamma} / \bar{\sigma}_{2 \gamma} \sim 0.006$, therefore making it unnecessary to consider single-photon annihilation any further. The small value of $\epsilon$ in (2.36) implies that $3 \gamma$ annihilation is a rare event that is only important when the selection rules forbid the $2 \gamma$ process (i.e. the self-annihilation of ortho-positronium). The singlet $2 \boldsymbol{\gamma}$-annihilation event is therefore predominant. Most positron experiments have concentrated on measuring the lifetimes and the energy and momentum distributions arising from this decay mode. The various annihilation modes are summarized in Table 2.1.

The probability of annihilation per unit time, or the annihilation rate $\lambda_{i}$, is the product of the relevant cross-section, the positron velocity and the number density $n_{e}$ of the 'available' electrons

$$
\tau_{i}^{-1}=\lambda_{i}=\bar{\sigma}_{i} v n_{e}
$$

where $\tau_{i}$ is the mean lifetime. Using the $2 \gamma$ cross-section in the slow positron limit (2.35) gives

$$
\lambda_{2 \gamma}=\pi r_{o}^{2} c n_{e}
$$

and $\lambda_{3 \gamma}=\epsilon \lambda_{2 \gamma}$. By measuring the annihilation rate one directly obtains an estimate of the electron density seen by the positron. However, the quantity $n_{e}$ must reflect several factors other than just the electron density and to emphasize this it is often written as $n_{e}=Z_{\text {eff }} n_{o}$, 
Table 2.1: The annihilation of electron-positron pairs: $\mathrm{e}^{+}+\mathrm{e}^{-}+x \mathrm{M} \rightarrow n \gamma+x \mathrm{M}$, where $n$ is the number of photons produced and $x$ is the number of other bodies (M) required to conserve momentum. $C P$ invariance requires slow $\mathrm{e}^{-}-\mathrm{e}^{+}$pairs to annihilate from either a ${ }^{1} S_{0}$ state with an even number of photons or a ${ }^{3} S_{1}$ state with an odd number of photons. Illustrative Feynman diagrams for each annihilation process are also shown. Filled-in vertices corresponding to momentum transfers between particles reduce the crosssection by $\alpha^{3}$. The open vertices correspond to photon creation and result in a factor of $\alpha$ in the cross-section. Annihilation processes beyond $3 \gamma$ have a factor of $\alpha^{n}$ in their cross-sections and have yet to be observed.

\begin{tabular}{|c|c|c|c|c|}
\hline$n$ & 0 & 1 & 2 & 3 \\
\hline$x$ & 2 & 1 & 0 & 0 \\
\hline Total spin, $S$ & 0 & 1 & 0 & 1 \\
\hline $\begin{array}{l}\text { Feynman } \\
\text { diagram }\end{array}$ & & & & $e^{+}$ \\
\hline Comments & $\begin{array}{l}\text { 4-body collision } \\
4 \text { vertices }-\alpha^{8} \\
\text { Never observed }\end{array}$ & $\begin{array}{l}\text { 3-body collision } \\
3 \text {-vertices }-\alpha^{5} \\
\text { Observed in high- } \\
\text { energy collisions }\end{array}$ & $\begin{array}{l}\text { 2-body collision } \\
2 \text {-vertices }-\alpha^{2} \\
\text { Most probable }\end{array}$ & $\begin{array}{c}2 \text {-body collision } \\
3 \text {-vertices }-\alpha^{3} \\
\bar{\sigma}_{2 \gamma} / \bar{\sigma}_{3 \gamma} \sim 378\end{array}$ \\
\hline
\end{tabular}

where $n_{o}$ is the actual atomic number density. $Z_{\text {eft }}$ is a parameter that describes the number of available electrons per atom seen by the positron and is likely to be quite different from the number of electrons per atom for a number of reasons - such as Coulomb forces, the polarizability of electrons and spin statistics. Coulomb repulsion from the nuclei inhibits positron annihilation with the inner core electrons. The polarizability of outer core electrons in insulators, or the Coulomb attraction between the positron and conduction electrons in metals, increases the instantaneous electron density near the positron site. These strong correlations result in a large electron-positron correlation energy and give rise to a sharp cusp in the electron density at the positron site. Since the annihilation rate is determined by the electron density at the positron, this cusp in the electron density must be understood in detail if useful information is to be extracted from positron data. These electron-positron correlation effects will be discussed in more detail in the next section.

In addition to the annihilation rates, the angular and energy distributions of the emitted $\gamma$-rays are also important characteristics of the annihilation process. Both of these distributions are affected by the motion of the electron-positron pair and therefore provide 
a)

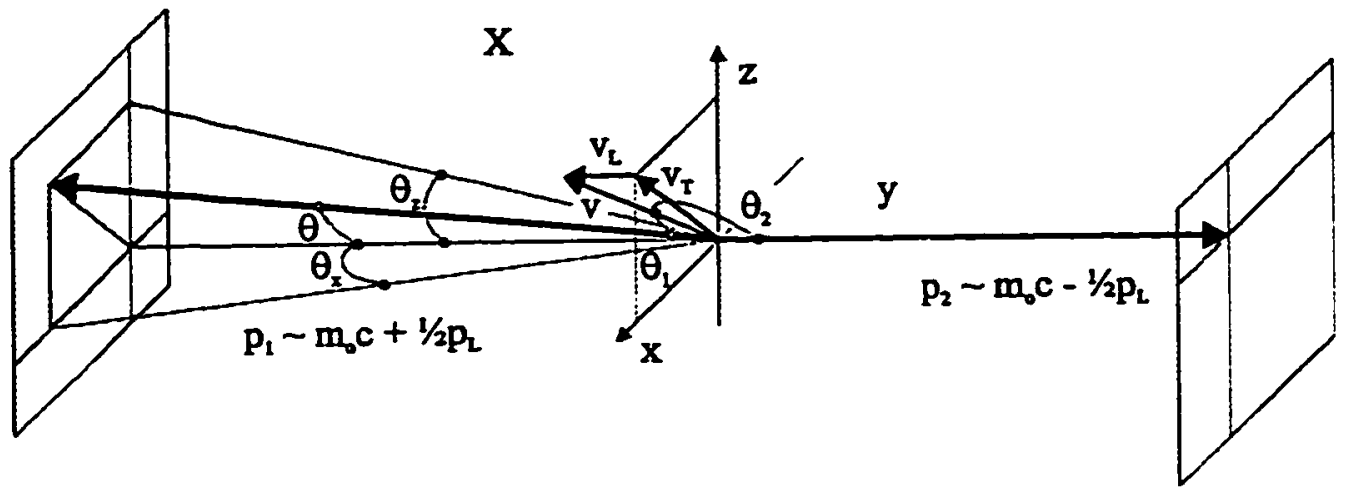

b)

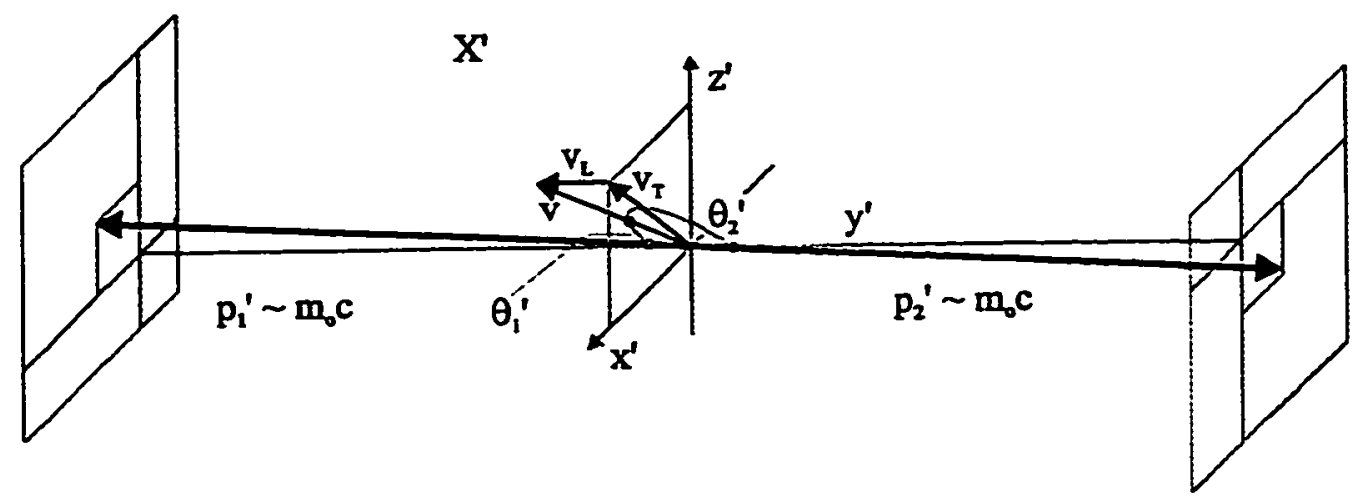

Figure 2.3: Two-photon annihilation in a) the laboratory reference frame $X$ in which the electron-positron pair has a velocity $v$ and $b$ ) the centre-of-mass frame $X^{\prime}$ which moves with velocity $v$ relative to $X$. In $X^{\prime}$ the pair is at rest. To conserve energy and momentum the two annihilation $\gamma$-rays must have exactly equal energies and equal and opposite momenta (i.e. $\theta_{+}^{\prime}+\theta_{-}^{\prime}=180^{\circ}$ ). In $X$ the motion of the electron-positron pair gives rise to small angular deviations from $180^{\circ}\left(\theta=180^{\circ}-\left(\theta_{+}+\theta_{-}\right)\right)$and a Doppler shift in the photon energies.

useful information about its state prior to annihilation. To understand this, consider the two reference frames shown in Figure 2.3. In the laboratory frame $X$, in which one of the $\boldsymbol{\gamma}$-rays is along the $y$-axis for simplicity, the pair moves with centre-of-mass velocity $\mathbf{v}$. The velocity can be written as $\mathbf{v}=v_{L} \hat{y}+v_{T} \hat{v}_{T}$, where the longitudinal component $v_{L}$ lies along the $y$-axis and the transverse component $v_{T}$ is in the $x z$-plane. $\theta_{1}$ and $\theta_{2}$ are the angles between $\mathbf{v}$ and the $\gamma$-ray wave vectors $\mathbf{k}_{+}$and $\mathbf{k}_{-}$, respectively. The centre-of-mass frame $X^{\prime}$ moves at $\mathbf{v}$ relative to $X$. By definition the electron-positron pair has zero total linear momentum in $X^{\prime}$ so for $2 \gamma$ annihilation, momentum conservation requires that the two photons have exactly equal $\left(p^{\prime} \approx m_{0} c\right)$ and opposite $\left(\theta_{+}^{\prime}+\theta_{-}^{\prime}=180^{\circ}\right)$ momenta, as shown in Figure 2.3b. From energy conservation each $\gamma$-ray has one-half the total energy of the system, i.e. $E^{\prime}=\frac{1}{2} E_{\text {tot }}^{\prime}=\frac{1}{2}\left(2 m_{o} c^{2}-E_{b}\right) \approx m_{o} c^{2} \approx 511 \mathrm{keV}$ where $E_{b}$ is the binding 
energy of the electron-positron pair. However, in the laboratory frame the motion of the electron-positron pair causes the $\gamma$-ray energies and emission angles $\left(\theta_{ \pm} \leq \theta_{ \pm}^{\prime}\right)$ to change. The few eV of kinetic energy of the pair results in a Doppler-shifted energy (see Appendix B)

$$
E_{ \pm} \approx E^{\prime} \pm \frac{\Delta E}{2} \approx m_{o} c^{2} \pm \frac{c p_{L}}{2}
$$

where $p_{z}$ is the momentum component of the electron-positron pair along the photon emission direction. By measuring the line shape of the annihilating $\gamma$-rays one obtains information about the $p_{\iota}$ momentum distribution. The $p_{\llcorner}$component gives rise to a $\gamma$-ray momentum of $p_{ \pm} \approx m_{0} c \pm p_{L} / 2$ in the laboratory frame. The momentum component $p_{T}$ transverse to the $\gamma$-ray emission direction produces a deviation from $180^{\circ}$ given by

$$
\theta=180^{\circ}-\left(\theta_{+}+\theta_{-}\right) \approx \frac{p_{T}}{m_{0} c}
$$

In this thesis, angular correlation experiments were performed in which the number of coincident $\gamma$-rays was measured as a function of $\theta$ to get information about the $p_{z}$ momentum distribution of the annihilating electron-positron pair. For electrons annihilating with thermalized positrons in condensed matter typical values for the momentum give rise to an energy difference between the two photons, $\Delta E$, of $\sim 2-5 \mathrm{keV}$ and an angular deviation from $180^{\circ}$ of $\sim 5-10$ mrads ( $1 \mathrm{mrad}$ corresponds to a momentum of $10^{-3} \mathrm{~m}_{0} c=0.137 \mathrm{a} . \mathrm{u}$.).

A derivation of relationships analogous to (2.39) and (2.40) for $3 \gamma$ annihilation is a far more difficult task since the energies and angles between photons are no longer unique in $X^{\prime}$. All that can be easily deduced is that when $v=0$ all three photons are emitted in the same plane. For $\mathbf{v} \neq 0$ the deviation of these angles from this plane is of order $\beta$. The energy spectrum for the $3 \gamma$ annihilation has been calculated by Ore and Powell (1949).

\subsubsection{2 $\gamma$ Annihilation in Condensed Matter}

In order to interpret the results of positron lifetime, Doppler broadening and angular correlation experiments it is necessary to have an expression for the probability that a positron immersed in an arbitrary system of electrons and external fields will annihilate into two $\gamma$-rays with total momentum $\mathbf{p}=\hbar \mathbf{q}$. Appendix $\mathrm{B}$ shows the probability to be

$$
\rho_{2 \gamma}(\mathbf{q})=\left.\frac{\pi r_{0}^{2} c}{(2 \pi)^{3}} \sum_{i}\left|\int_{V} e^{-i q \cdot r} \Psi_{e p, i}(r, r) d r\right|\right|^{2}
$$


where $\Psi_{e p, i}\left(r_{e}, r_{p}\right)$ is an electron-positron pair wave function for an electron in initial state $i$ at $r_{e}$ and a thermalized positron at $r_{p}$. The summation in (2.41) runs over all occupied electronic states $i$. For calculations, the electronic states are generally separated into core states ( $i_{c}=n, l, m$ where $n, l$, and $m$ are the usual atomic quantum numbers) and valence states ( $i_{v}=\mathbf{k} j$ where $\mathbf{k}$ and $j$ are the Bloch wave vector and the band index). For obvious reasons $(2.41)$ is often referred to as the momentum density of the photon-pair. As mentioned above, doppler broadening and angular correlation experiments measure various components of this momentum density. Lifetime experiments are unable to discriminate the photon momentum and give only the total annihilation rate

$$
\begin{aligned}
\lambda_{2 \gamma} & =\int \rho_{2 \gamma}(\mathbf{q}) d \mathbf{q} \\
& =\pi r_{o}^{2} c \int \sum_{i}\left|\Psi_{e p, i}(\mathbf{r}, \mathbf{r})\right|^{2} d \mathbf{r}
\end{aligned}
$$

where (2.42a) and (2.42b) are related by Parseval's identity.

The interactions of a positron in a many-electron system are significantly different from those of an electron interacting with the same system. For electrons, the Coulomb repulsion acts to reduce the electron density from its average value near each electron each electron is said to carry a 'correlation hole'. Similarly, Pauli exclusion causes each electron to carry an 'exchange hole' around it that is free of other electrons with the same spin state. Both of these effects reduce the electron-electron interaction energy and results in an electron density that has a minimum at each electron site and increases smoothly to its average value between electrons. The situation, however, is quite different for a positron. Since there is generally only one positron in the system at a time, it can drop to the lowest positron state. The Coulomb attraction between the positron and the neighbouring electrons increases the instantaneous electron density near the positron site. In metals, the conduction electrons form a screening cloud around the positron resulting in densities at the positron site that are often 5-10 times the average value. This increase in density results in a shorter positron lifetime. Due to their tight-binding nature, the core electrons are more strongly dominated by the screened Coulomb potential of their nuclei and are therefore much less able to respond to the small perturbation caused by the screened-out positron. The situation in insulators is quite different. In this case, the positron is no longer surrounded by easily perturbable conduction electrons - any density enhancement must come from the polarization of the outer core electrons. The screening of the positron is no longer isotropic and the density enhancements are generally thought to be quite small 
(i.e. < 1.5). It is rather surprising therefore that density enhancements at the positron site of about 3-5 are required in the condensed rare gases to make theory correspond to experiment.

The strong electron-positron correlations make it difficult to construct an accurate two-particle wave function as required by (2.41) and (2.42). Several calculations have tried to describe the electron density enhancements by including the correlation effects directly in the two-particle wave function. Two attempts relevant to positron-core electron annihilations are mentioned in §2.5.4. However, these calculations are, by necessity, difficult and require significant simplifications to make the calculations tractable. One simpler approach has been to write the pair wave function in terms of one-particle wave functions

$$
\Psi_{e p, i}(\mathbf{r}, \mathbf{r})=\Psi_{p}(\mathbf{r}) \Psi_{e, i}(\mathbf{r}) \sqrt{g(\mathbf{r}, i)}
$$

where $\Psi_{p}(r)$ is an unperturbed positron wave function, $\Psi_{e, i}(\mathbf{r})$ is an unperturbed oneelectron wave function (i.e. no positron present) and $g(\mathbf{r}, i)$ is an electron-positron correlation function. The single-particle wave functions can be obtained from band structure calculations like those described in $§ 2.4 .1$. The correlation functions are meant to describe both the distortion of the positron wave function from its original shape and the density enhancement of the $i$ electronic state at the positron site. The determination of these functions for a positron immersed in condensed matter is still a complicated and unsolved many-body problem. Therefore, the $g(r, i)$ are typically described using various theoretical and/or semi-empirical approximations. The approximations relevant to this work will be described in $\$ 2.5 .4$. However, it convenient to first describe simplifications to (2.41) that occur when dealing only with positron-core electron annihilations.

\subsubsection{Core-Electron Annihilation}

In general, different 'types' of electrons gives rise to different momentum distributions and positron annihilation rates. In metals, the positron annihilates predominately with conduction electrons despite the fact that the ionic core electrons are generally far more numerous. The reason is that the positron feels a strong Coulomb repulsion from the nuclei which keeps it out of the core region, thereby reducing the positron-core electron wave function overlap and the corresponding contribution to the momentum density and the annihilation rate. However, in insulating materials the absence of conduction electrons 
makes the annihilation with core electrons the dominant mode of positron decay. In many respects, the condensed rare gases studied in this work are prototype materials for studying positron-core electron annihilations. In the rare gases, all electrons are core electrons with a filled-shell configuration that offers calculational advantages. Furthermore, the atoms are bound by van der Waal's forces so that each Wigner-Seitz cell is electrically neutral. Therefore, no complications arise from positron-ion core correlations that might be expected in ionic or covalent binding media.

The simplest description of positron-core electron annihilation, and the one most often used, is obtained using the tight-binding approximation for the electron wave functions. In this approximation the Bloch core-electron wave functions are written in terms of atomic orbitals $\phi_{\mathrm{n} l m}$ localized about the lattice sites $R_{\nu}$ and modulated by a phase factor

$$
\Psi_{e, n l m k}(\mathbf{r})=\sum_{\nu} e^{i \mathbf{k} \cdot \mathbf{R}_{\nu}} \phi_{n l m}\left(\mathbf{r}-\mathbf{R}_{\nu}\right)
$$

where the $\phi_{n l m}(\mathbf{r})$ are atomic wave functions given by (2.14), with the radial functions $U_{n l}(r)$ obtained from the Hartree-Fock approximation. $\mathbf{k}$ is the crystal momentum inside the first Brillouin zone. Now insert (2.43) and (2.44) into (2.41), and assume that the overlap between neighbouring sites is negligible (i.e. $\int \phi^{-}(\mathbf{r}) \phi\left(\mathbf{r}-\mathbf{R}_{\nu}\right) d \mathbf{r} \ll 1, \mathbf{R}_{\nu} \neq 0$ ). Using this and the addition theorem for spherical harmonics gives the positron-core electron momentum density as the sum over the occupied core bands labeled by $n l$

$$
\begin{aligned}
\rho_{2 \gamma, c}(q) & =\sum_{n l} \rho_{n l}(q) \\
& =\frac{\pi r_{0}^{2} c}{(2 \pi)^{3}} \sum_{n l} 8 \pi(2 l+1)\left|\int_{0}^{\infty} \Psi_{p}(r) U_{n l}(r) j_{l}(q r) \sqrt{g(r, n l)} r^{2} d r\right|^{2}
\end{aligned}
$$

where a factor of two for spin degeneracy has been included and the positron wave function has been assumed to be isotropic. $j_{l}$ is the $l^{\text {th }}$ spherical Bessel function. This result was first derived by Berko and Plaskett (1958). Similarly, starting from (2.42), the tight binding core annihilation rate can be shown to be

$$
\begin{aligned}
\lambda_{2 \gamma, c}(q) & =\sum_{n l} \lambda_{n l} \\
& =\pi r_{0}^{2} c \sum_{n l} 2(2 l+1) \int_{0}^{\infty}\left|\Psi_{p}(r)\right|^{2}\left|U_{n l}(r)\right|^{2} g(r, n l) r^{2} d r \\
& =\pi r_{0}^{2} c \int_{0}^{\infty}\left|\Psi_{p}(r)\right|^{2} n_{c}(r) g(r, n l) 4 \pi r^{2} d r
\end{aligned}
$$

where $\lambda_{n l}$ corresponds to the annihilation rate resulting from the $n l^{\text {th }}$ core shell and $n_{c}(r)$ is the core electron density. 
The condensed rare gases represent the limiting case for the applicability of the tightbinding model in the sense that the ionic core electrons in metals are generally more confined than the core electrons in the corresponding rare-gas solid. Therefore, if the tight binding model proves useful in the rare gases then it can be applied to core electron annihilation in metals with even greater assurance.

\subsubsection{Positron-Core Electron Correlations}

The simplest and most common approximation used in positron studies is the independent particle model (IPM). In this case the electron-positron correlations are simply ignored, i.e. $g(\mathbf{r}, i) \boxminus 1$ in (2.43) and (2.45). This essentially turns off the attractive Coulom$b$ field between the positron and the electrons so there is no density enhancement at the positron site. In this case, $(2.41)$ and $(2.42 b)$ reduce to the well-known IPM expressions

$$
\rho_{\mathrm{IPM}}(\mathbf{q})=\frac{\pi r_{o}^{2} c}{(2 \pi)^{3}} \sum_{i}\left|\int_{V} e^{-i \mathbf{q} \cdot \mathbf{r}} \Psi_{p}(\mathbf{r}) \Psi_{e, i}(\mathbf{r}) d \mathbf{r}\right|^{2}
$$

which can be inserted into $(2.42 \mathrm{a})$ to give

$$
\lambda_{\mathrm{IPM}}=\pi r_{o}^{2} c \int_{V}\left|\Psi_{p}(\mathbf{r})\right|^{2} \sum_{i}\left|\Psi_{e, i}(\mathbf{r})\right|^{2} d \mathbf{r}
$$

The IPM is the usual starting point for most positron physics investigations. Within this model electron-positron correlation effects are described using a momentum-dependent enhancement factor

$$
\epsilon_{\mathrm{corr}}(\mathbf{q})=\rho_{2 \gamma}(\mathrm{q}) / \rho_{\mathrm{IPM}}(\mathbf{q})
$$

and an average enhancement factor

$$
\gamma_{\mathrm{corr}}=\lambda_{2 \gamma} / \lambda_{\mathrm{IPM}}
$$

In many condensed media (2.50) is often a reasonable first approximation to the actual shape of the momentum density as a result of a weak momentum dependence of the correlation functions. However, by ignoring the electron-positron correlations the IPM fails to predict the area under the momentum density (i.e. $\lambda_{2 \gamma}$ ) sometimes by as much as a factor of 5 or more. The simultaneous interpretation of positron lifetime and momentum density measurements therefore requires that the correlations be properly accounted for. 
Two different approaches have been used to include positron-electron correlation effects: to include the correlations directly in the two-particle wave function as in (2.54) below; or to write the two-particle wave function in terms of one-particle wave functions and a correlation function as was done in (2.43). The first serious attempt to calculate a positron-core electron pair wave function that included the correlations directly was that of Woll and Rose (1966). Their technique was based on the Born-Oppenheimer methods for calculating molecular properties. Despite their rather general approach, they were forced to make a number of significant approximations, the effects of which are not obvious, to keep the computations tractable. When applied to solid argon, this approach gave results that were in significant disagreement with experiment.

A second attempt to include the correlations in the pair wave function to describing core-electron annihilation in metals and nonionic insulators is due to Carbotte (Carbotte 1966; Carbotte and Salvadori 1967; Salvadori and Carbotte 1969). This approach extended an approximation scheme originally developed by Kahana (1963) for positron annihilations in an electron gas to core electrons. The theory is based on the selective summation of ladder diagrams in the perturbative expansion of the electron-positron twoparticle Green's function. It was found that the main correction for the correlation effects could be included by writing the pair wave function as

$$
\Psi_{e p, n l m k}\left(r_{e}, r_{p}\right)=\Psi_{p}\left(r_{p}\right) \Psi_{e, n l m k}\left(r_{e}\right)+\phi_{e p}\left(r_{e}, r_{p}\right)
$$

where $\Psi_{p}\left(\mathbf{r}_{p}\right) \Psi_{e, n l m k}\left(\mathbf{r}_{e}\right)$ is the same as the IPM result and $\phi_{e p}\left(\mathbf{r}_{e}, \mathbf{r}_{p}\right)$ is the correlation term. After applying a number of simplifications to the correlation term, expressions for the momentum density and annihilation rate were derived. Sharma and Majumdar (1979) have applied this theory, with corrections to several calculational errors, to the heavier rare gas solids. Their results, discussed in $§ 5.1 .2$, still give unsatisfactory agreement with experiment.

Chiba et al. (1977) have used the basic Carbotte theory, with small modifications, to derive very simple expressions for the momentum dependent enhancement factors. In this case, the momentum density for core states is written as

$$
\rho_{2 \gamma, c}(q)=\sum_{n l} 2(2 l+1) \epsilon_{\mathrm{corr}, n l} \rho_{\mathrm{IPM}, n l}(q)
$$


For localized electron states the enhancements are written as

$$
\epsilon_{\mathrm{corr}, n l}=\rho_{n l}(q) / \rho_{\mathrm{tPM}, \mathrm{nl}}=1+h^{2}\left(q, \Delta_{n l}\right)
$$

while for Bloch electron states

$$
\epsilon_{\mathrm{corr}, n l}=\left(1+h\left(q, \Delta_{n l}\right)\right)^{2}
$$

where $\Delta_{n l}$ is the binding energy of an electron in the $n l$ core band and $h\left(q, \Delta_{n l}\right)$ is

$$
\begin{aligned}
h\left(q, \Delta_{n l}\right)=\frac{1}{q}\left(\pi \theta\left(\frac{1}{2}-\Delta\right)\right. & -\tan ^{-1}\left(\frac{1}{\sqrt{1+2 \Delta}-2 \sqrt{\Delta}}\right) \\
& \left.-\tan ^{-1}\left(\frac{1}{\sqrt{1+2 \Delta}+2 \sqrt{\Delta}}\right)\right)
\end{aligned}
$$

where $\Delta=2 \Delta_{n l} / q^{2}$ and $\theta(x)$ is the unit step function. Both (2.57) and (2.58) are in atomic units. Sharma and Singh (1982) have applied these enhancements to solid argon and xenon and achieved only slightly better agreement with experiment. However, Bonderup et al. (1979) have observed that one of the approximations used to simplify the correlation term in (2.54) was inappropriate and had the effect of significantly overestimating the core-electron enhancements. While Carbotte's basic theory is correct, the validity of the work described above, all of which suffers from the same inappropriate approximation, must be questioned. Without this approximation, obtaining useful expressions for the momentum density and annihilation rate within this theory becomes prohibitively difficult, making it necessary to search for simplified schemes to describe the core-electron enhancements.

The enhancements due to positron-conduction electron correlations have been studied extensively both experimentally and theoretically. Most theoretical discussions have focused on the model of a single positron in an interacting electron gas (i.e. the positronelectron jellium model). Only a few efforts have been made to solve the much more difficult problem of correlations in the presence of a crystal lattice. The electron-gas results have been applied with good success to the conduction electrons in nearly-free-electron metals in which the lattice has a small effect, such as some of the alkali metals and aluminum. Variations of these models have also been applied, with varying degrees of success, to the more difficult cases of transition metals and semiconductors.

Unfortunately, there has been significantly less study of the positron-core electron correlations. Most studies have simply ignored these correlations or, at best, applied a small, constant, momentum-independent enhancement factor. The reason for this is that it 
is generally believed that for very tightly bound electrons (i.e. at high momentum, $\geq 15 \mathrm{~m}$ rads) the enhancement factors should be $\sim 1-1.5$. Bonderup et al. (1979) have shown that for a large relative velocity $v$ between the annihilating electron and positron, as compared to the Bohr velocity $v_{o}=e^{2} / \hbar$, the enhancement factor is small and is approximately

$$
\epsilon_{\mathrm{corr}}(q) \approx 1+\pi v_{o} / v
$$

The condition that $v_{o} \ll v$ is certainly reasonable for the inner core electrons. However, Daniuk et al. $(1989,1991,1992)$ have observed that at the beginning of each series of metals ( $\mathrm{Na}$ to $\mathrm{Al}, \mathrm{K}$ to $\mathrm{Zn}, \mathrm{Rb}$ to $\mathrm{Cd}$ ) the average core enhancement factor is significantly larger than 1 in the low-momentum regions. Thus, while small, constant core enhancement factors may be sufficient to describe the results in $\mathrm{Al}$ or $\mathrm{Cu}$, they are completely unsatisfactory for the alkali metals. As will be seen, this is also true for the condensed rare gases.

Recently several groups have started to use the correlation function approach described by (2.43) and apply versions of the electron-gas correlation functions to describe the positron-core electron enhancements. That is, positron annihilations with core electrons are simply considered as annihilations in a high-density electron gas. These attempts have met with some reasonable success and are nicely summarized by Daniuk et al. (1991).

One enhancement scheme that has been used to describe positron-core electron correlations in various metals is that of Jarlborg and Singh (1987). Their enhancement is obtained by solving a two-body Schrödinger equation for the electron and positron which are considered to interact inside a sphere of radius

$$
r_{s}(\mathbf{r})=\left(\frac{3}{4 \pi n(\mathbf{r})}\right)^{1 / 3}
$$

where $n$ is the electron density. $r_{s}$ defines an approximate radius for the exchange-correlation hole around the electrons. The $\mathrm{e}^{-}-\mathrm{e}^{+}$interaction is then described in the local density approximation as an impurity problem defined inside a Wigner-Seitz sphere of radius $r_{s}$. The square of the resulting enhancement factor $\epsilon^{2}\left(r_{s}(r)\right)=g(r, i)$ is equal to the pair-correlation function described in (2.43). The enhancement factor is reasonable except for large values of $r_{s}$ (i.e. very low electron densities) or for the innermost core-electron levels where the wave functions can have a significant variation across the exchange-correlation hole. The problem with the inner core levels can be ignored since these levels have a negligible overlap with the positron. Jarlborg and Singh (1987) have tabulated their enhancement as a function of $r_{s}$ using a reduced mass of 1 . These results were modified to correspond to the more natural 
choice of $\frac{1}{2}$ for the reduced mass and then fit using a simple polynomial to give

$$
\epsilon\left(r_{s}\right)=1.0028+0.3430 r_{s}+0.0056 r_{s}^{2}+0.0258 r_{s}^{3}
$$

where $r_{s}$ is in atomic units. This equation corresponds quite closely to the enhancements calculated by Rubaszek et al. (1984) using the Kahana formalism extended to a high-density electron gas. It is also similar to the interpolation formula proposed by Boronski and Nieminen (1985) for one position in a homogenous electron gas

$$
\epsilon\left(r_{s}\right)=1+1.23 r_{s}+0.8295 r_{s}^{3 / 2}-1.26 r_{s}^{2}+0.3286 r_{s}^{5 / 2}+\frac{1}{6} r_{s}^{3}
$$

For $r_{s}<2(2.62)$ predicts an enhancement that is slightly larger than (2.61) while for $r_{s}>2$ (2.62) gives a slightly smaller enhancement. Either of these formulas inserted into (2.43) should produce estimates for the angular correlation curves and positron lifetimes that are a significant improvement over the IPM results. 


\section{Chapter 3}

\section{Positronium Physics and Chemistry}

Following the discovery of the positron, Mohorovičic (1934) suggested that a positron might bind an electron to form a neutral quasi-stable atom. In an unsuccessful attempt to observe the spectroscopic structure of this bound state Ruark (1945) named it positronium (Ps). The first experiments indicating the possibility of Ps formation were those of Shearer and Deutsch (1949). Later experiments by Deutsch (1951a, 1951b) firmly established the existence of Ps in several gases. Since then Ps has been observed in many other gases, in a variety of condensed media as well as in interstellar space. The advances in the understanding of the Ps system and its use to explore the basic structure of QED has been summarized in a number of reviews (Stroscio 1975, Berko and Pendelton 1980, Rich 1981).

The aim of this chapter is to describe the interesting, and often complicated, history of Ps immersed in a condensed matter system. It is useful to begin with a description of an isolated Ps atom, which is done in $§ 3.1$. Section 3.2 discusses some of the changes required to describe Ps immersed in condensed matter. Given this background, it is possible to start tracing positronium's history in condensed matter. Section 3.3 discusses the two main models used to describe Ps formation - the 'Ore gap' and positron spur models. Once Ps thermalizes it can exist in a either a delocalized or a localized state. In liquids, the repulsive interaction between the medium and Ps causes it to become localized in a selftrapped bubble state. In the rare-gas solids it appears that Ps annihilates from inside defects. The various possible Ps states are described in §3.4. Section 3.5 discusses several physical and chemical processes that can have a significant effect on the Ps annihilation characteristics. In this work, both magnetic and electric fields were applied to condensed samples and provided some important insight into Ps formation and its interactions. A simple model to describe Ps spin-exchange reactions with spur electrons is developed in §3.5.3.1. Finally, the effects of $\mathrm{O}_{2}$ impurity in a sample is discussed in §3.5.3.2. These ideas are used to describe the results of several experiments in which small amounts of $\mathrm{O}_{2}$ impurity were deliberately added to liquid argon and $\mathrm{N}_{2}$. 


\subsection{Properties of an Isolated Positronium Atom}

The non-relativistic quantum mechanics for an isolated Ps atom in vacuum is nearly identical to that for a hydrogen atom. In the absence of any external interactions the Ps spatial wave function, $\Psi_{\mathrm{Ps}_{s}}^{\mathrm{vac}}\left(\mathbf{r}_{e}, \mathbf{r}_{p}\right)$, can be factored into a function $\psi_{\mathrm{P}_{5}}^{\mathrm{vac}}(\underline{\varrho})$ depending only on the relative electron-positron coordinate $\varrho=r_{e}-r_{p}$, times a function $X_{\mathbf{p}_{s}}^{\mathrm{vac}}(\mathbf{R})$ depending only on the centre-of-mass coordinate $\mathbf{R}=\frac{1}{2}\left(\mathbf{r}_{e}+\mathbf{r}_{p}\right)$. For isolated Ps, the centre of mass moves like a free particle. The only change required to the centre-of-mass Schrödinger equation for hydrogen is to replace the hydrogen reduced mass $\left(\mu_{H} \approx m_{e}\right)$ with that for Ps $\left(\mu_{\mathrm{Ps}}=m_{c} / 2\right)$

$$
\left(-\frac{\hbar^{2}}{2 \mu_{\mathrm{Ps}}} \nabla^{2}-\frac{e^{2}}{4 \pi \epsilon_{o} \varrho}\right) \psi_{\mathrm{Ps}_{\mathrm{s}}}^{\mathrm{vac}}(\varrho)=E_{\mathrm{Ps}}^{\mathrm{vac}} \psi_{\mathrm{Ps}}^{\mathrm{vac}}(\varrho)
$$

Since the orbital, spin and total angular momentum operators $\hat{\mathbf{L}}^{2}, \hat{\mathbf{L}}_{z}, \hat{\mathbf{S}}^{2}, \dot{\mathbf{S}}_{z}, \hat{\mathbf{J}}^{2}$, and $\overline{\mathbf{J}}_{z}$ all commute with (3.1), it follows that the wave functions and energies can be labeled using the usual spectroscopic notation. The solution to (3.1) gives a ground state spatial wave function

$$
\Psi_{\mathrm{Ps}, 1 s}^{\mathrm{vac}}(\varrho)=\frac{1}{\sqrt{8 \pi a_{o}^{3}}} e^{-\varrho /\left(2 a_{o}\right)}
$$

where $a_{o}$ is the Bohr radius and the most probable electron-positron separation is $2 a_{o}$. Since each particle moves about the centre of mass, this is approximately the Ps atom's diameter - i.e. Ps is the same size as a hydrogen atom. The non-relativistic energy levels have absolute values that are half those of hydrogen

$$
E_{\mathrm{Ps}, n}^{\mathrm{vac}}=-\frac{\mu_{\mathrm{Ps}} c^{2} \alpha^{2}}{2 n^{2}}=-\frac{m_{e} c^{2} \alpha^{2}}{4 n^{2}}
$$

where $n=1,2, \ldots$ is the principle quantum number. From (3.3) the ground state binding energy for Ps in vacuum is $E_{\mathbf{P}_{\mathbf{s}, \mathrm{b}}^{\mathrm{vac}}}^{\mathrm{P}}=6.803 \mathrm{eV}$.

The relativistic corrections to (3.1) introduce a splitting into the energy levels i.e. the appearance of a fine structure. These corrections include purely orbital terms as well as spin-orbit, spin-spin and annihilation interactions (Berestetskii et al. 1982). Since the orbital and spin momenta are coupled, $\hat{\mathrm{L}}^{2}, \hat{\mathbf{L}}_{z}$ and $\hat{\mathrm{S}}_{z}$ are no longer constants of the motion. To complete the set of commuting observables it is useful to choose the spatial and spin parts of the Ps wave function to be eigenstates of the parity operator $\hat{\mathbf{P}}$ and the spin exchange operator $\hat{\boldsymbol{\Sigma}}$, respectively (see Appendix B). It follows that the Ps wave functions 
also have a definite charge parity as defined by (B.1). Using this set of commuting variables, which is convenient for the study of the relativistic corrections to (3.3), annihilation rates and the effects of external fields, the Ps energy levels can be classified by the total spin or, equivalently, by $\Sigma$. If the $z$ component of the electron and positron spin are denoted by $\uparrow(\downarrow)$ and $f(t)$ respectively for $m_{s}=+\frac{1}{2}\left(-\frac{1}{2}\right)$ then the spin-spin basis vectors for the Ps ground state are $|\uparrow \downarrow\rangle, \| \downarrow\rangle,|\downarrow t\rangle$ and $\| \uparrow\rangle$. As in hydrogen and helium, the Ps ground state spin eigenvectors $\left\{S, m_{s}\right\rangle$ for $\dot{\mathbf{S}}^{2}$ and $\dot{\mathrm{S}}_{z}$ are the singlet $\left(1^{1} S_{0}, S=0, \Sigma=-1\right)$ state

$$
|0,0\rangle=\frac{1}{\sqrt{2}}(|\uparrow t\rangle-|\downarrow \neq\rangle)
$$

and the three triplet $\left(1^{3} S_{1}, S=1, \Sigma=+1\right)$ states

$$
\begin{aligned}
|1,-1\rangle & =|\downarrow t\rangle \\
|1,0\rangle & =\frac{1}{\sqrt{2}}(|\uparrow \downarrow\rangle+|\downarrow \neq\rangle) \\
|1,+1\rangle & =|\uparrow \neq\rangle .
\end{aligned}
$$

Singlet states are called parapositronium ( $p$-Ps) while the triplet states are called orthopositronium (o-Ps). The relativistic corrections for Ps are significantly different from those for hydrogen not only in scale but also in general structure. The large magnetic moment of the positron, as compared to the proton, makes the magnetic spin-spin interaction in Ps comparable to its spin-orbit interaction - i.e. the Ps hyperfine splitting is of the same order as its fine structure splitting. Virtual annihilation mechanisms cause spindependent fine-structure shifts comparable to those caused by the spin-orbit and spin-spin interactions. The calculated theoretical value for the splitting of the $n=1$ Ps levels is $\Delta E_{\mathrm{Ps}, 1}^{\mathrm{vac}}=E_{o-\mathrm{Ps}, 1}^{\mathrm{vac}}-E_{p-\mathrm{Ps}, 1}^{\mathrm{vac}}=203400.3 \mathrm{MHz}\left(\sim 8.41 \times 10^{-4} \mathrm{eV}\right)$ which agrees reasonably well with the best experimental value of $203389.1 \pm 0.7 \mathrm{MHz}$ (Ritter et al. 1984). This splitting is very small compared to the Ps binding energy and implies that $p$-Ps and $o$-Ps will be formed in a ratio of their statistical weights of $\frac{1}{4}$ and $\frac{3}{4}$, respectively.

Positronium is quasi-stable in that it annihilates after a finite period of time. An isolated Ps atom in vacuum must self-annihilate - i.e. the positron annihilates with the electron to which it is bound. Since the electron and positron momenta in Ps are small compared to $m_{\mathrm{e}} c$ their annihilation can, to lowest order, be treated as the annihilation of a slow electron-positron pair, as discussed in §2.5.1. As with slow electron-positron pairs the total spin-averaged Ps self-annihilation rate, $\lambda_{\mathbf{P s}_{\mathbf{s}}^{\text {vac }}}^{\text {, is }}$

$$
\lambda_{\mathrm{Ps}}^{\mathrm{vac}}=\frac{1}{4} \lambda_{1}^{\mathrm{vac}}+\frac{3}{4} \lambda_{3}^{\mathrm{vac}}=\lambda_{2 \gamma}+\lambda_{3 \gamma}+\lambda_{4 \gamma}+\cdots
$$


where the self-annihilation rates for $p$-Ps and o-Ps in vacuum, $\lambda_{1}^{\text {vac }}$ and $\lambda_{3}^{\text {vac }}$, have been weighted by their multiplicities. Recalling the effects of charge parity conservation and comparing (3.5) with (B.3) shows that

$$
\lambda_{1}^{\mathrm{vac}} \approx 4 \lambda_{2 \gamma}, \quad \lambda_{3}^{\mathrm{vac}} \approx \frac{4}{3} \lambda_{3 \gamma}
$$

where the $4 \gamma$ and higher annihilation processes have been ignored. Lepage et al. (1983) have shown this to be reasonable.

Using (2.37) the p-Ps self-annihilation rate is

$$
\lambda_{1}^{\mathrm{vac}}=4\left(v \bar{\sigma}_{2 \gamma}\right)_{v \rightarrow 0}\left|\psi_{\mathrm{Ps}, 1 s}^{\mathrm{vac}}(0)\right|^{2}
$$

where $n_{e}$ is replaced by the electron density at the positron - i.e. $\left|\psi_{\mathrm{Ps}, 1 s}^{\mathrm{vac}}(0)\right|^{2}=1 /\left(8 \pi a_{o}^{3}\right)$. Inserting (2.35) into (3.7) predicts a p-Ps self-annihilation rate of $8.03 \mathrm{~ns}^{-1}$, corresponding to a lifetime of $\tau_{1}^{\text {vac }}=0.123 \mathrm{~ns}$. When radiative corrections are included $\lambda_{1}^{\text {vac }}=7.9852 \mathrm{~ns}^{-1}$ $\left(\tau_{1}^{\text {vac }}=0.1252 \mathrm{~ns}\right)$ which compares well with the experimental value of $7.994 \pm 0.011 \mathrm{~ns}^{-1}$ (Gidley et al. 1982). The level width for $p$-Ps (and o-Ps) is small compared to the groundstate energy, i.e. $\hbar / \tau_{\mathrm{L}}^{\mathrm{vac}} \ll E_{\mathrm{Ps}, 1}^{\mathrm{vac}}$, so positronium may be regarded as being in a quasistationary state.

Similarly, the $o$-Ps self-annihilation rate is

$$
\lambda_{3}^{\mathrm{vac}}=\frac{4}{3}\left(v \bar{\sigma}_{3 \gamma}\right)_{v \rightarrow 0}\left|\psi_{\mathrm{Ps}, 1 s}^{\mathrm{vac}}(0)\right|^{2}
$$

which predicts $\lambda_{3}^{\text {vac }}=7.211 \mu \mathrm{s}^{-1}\left(\tau_{3}^{\text {vac }}=138.6 \mathrm{~ns}\right)$. Radiative corrections give $\lambda_{3}^{\text {vac }}=$ $7.03830 \pm 0.00007 \mu \mathrm{s}^{-1}$ corresponding to a lifetime of $\tau_{3}^{\text {vac }}=142.08 \mathrm{~ns}$ (Adkins 1983). The best experimental measurement to date of $\lambda_{3}^{\text {vac }}$ is $7.0514 \pm 0.0014 \mu \mathrm{s}^{-1}$ (Westbrook et al. 1989) exceeds the theoretical value by nearly ten standard deviations. This remains one of the important, unsolved discrepancies in quantum electrodynamics. Attempts to find more exotic o-Ps decay modes to explain this discrepancy have so far been unsuccessful (Asai et al. 1991; Gidley et al. 1991).

\subsection{Positronium in Condensed Matter}

The identification and description of positronium in condensed matter is a somewhat more difficult task. One reasonable definition for Ps in condensed matter is that the 
entire one positron, many-electron system should have different spin states with distinct annihilation rates similar to those for a Ps atom in vacuum (Dupasquier 1988). By this definition, electrons from fully-closed shells cannot bind to a positron to form Ps - there has to be at least one unpaired electron which can couple to the positron in either a singlet or a triplet state. This coupling is assumed to be the dominant factor - however, it is understood that the presence of the other electrons and the internal fields of the medium can have significant effects on the Ps spatial wave function, the energy levels and binding energy, and the annihilation rates. Using the above criterion, positronium-like states have not been observed in metals or semiconductors. In metals, for instance, the conduction electron density is usually high and the ccrrelation between the positron and a single electron lasts for only a very short time $\left(\sim 10^{-16} s\right)$. The positron is therefore considered to remain essentially free and is instead 'shared' amongst the screening cloud of conduction electrons. This is in contrast to many insulating materials where an appreciable fraction of the positrons $(\sim 10-70 \%)$ form Ps-like states with distinct annihilation rates for the singlet and triplet states.

The Ps annihilation rates are strongly affected in the presence of a medium. Firstly, the exchange and electrostatic forces encountered by the bound electron-positron pair in a medium always tends to separate the two particles, perturbing their spherical symmetry to create an 'elongated' Ps. This distortion of the Ps spatial wave function reduces the overlap probability of the particles thereby reducing their self-annihilation rate. Secondly, the positron in the Ps now has a non-zero overlap with electrons in the medium other than the one to which it is bound and can therefore annihilate with them in a process known as 'pick-off' annihilation. This process is especially important for o-Ps where the positron in Ps can now undergo a fast $2 \gamma$ annihilation by overlapping with a 'foreign' electron in a spin-singlet state. Pick-off annihilation typically reduces the $o$-Ps lifetime from the 142 ns vacuum value to just a few nanoseconds in condensed matter. The effect on $p$-Ps is much less dramatic as its self-annihilation rate is already quite fast.

To understand the effects of the medium consider a bound electron-positron pair immersed in a diamagnetic insulating medium. It is reasonable to assume that the positron is strongly correlated to only one electron at a time and that the remaining $n$ electrons are tightly bound with full closed-shell symmetry. Following Hodges et al. (1972), the wave function describing the system can be formed using a properly antisymmetrized product of the Ps wave function $\Psi_{\mathbf{P s}_{s}}\left(r_{e}, r_{p}\right)$, times the core electron Bloch functions which form an 
orthonormal set. The Ps wave function is easily made orthogonal to the $\Psi_{k_{j}}\left(r_{e}\right)$ over the electron coordinates for all $\mathbf{r}_{p}$ (i.e. $\left\langle\Psi_{\mathrm{Ps}_{s}}\left(\mathbf{r}_{e}, \mathbf{r}_{p}\right) \mid \Psi_{\mathbf{k} j}\left(\mathbf{r}_{e}\right)\right\rangle=0$ ) without changing the value of the Slater determinant by using the Gram-Schmidt orthogonalization process. Doing this results in particularly simple expressions, free of exchange terms, for the Ps momentum densities and the self-annihilation and pick-off annihilation rates. The momentum density for the $2 \gamma p$-Ps self-annihilation becomes

$$
\rho_{\mathrm{l}, \mathrm{sa}}(\mathbf{q})=\frac{4 \pi r_{o}^{2} c}{8 \pi^{3}}\left|\int_{\Omega} \Psi_{\mathrm{Ps}_{\mathbf{s}}}(\mathbf{r}, \mathbf{r}) \mathrm{e}^{-i \mathbf{q} \cdot \mathbf{r}} d \mathbf{r}\right|^{2}
$$

while for the $2 \gamma$ pick-off annihilations

$$
\rho_{\mathrm{po}}(\mathbf{q})=\frac{\pi \tau_{o}^{2} c}{8 \pi^{3}} \sum_{\mathbf{k} j} \int_{\Omega} d \mathbf{r}_{e}\left|\int_{\Omega} \Psi_{\mathrm{Ps}}\left(\mathbf{r}_{e}, \mathbf{r}\right) \Psi_{\mathbf{k} j}(\mathbf{r}) \mathrm{e}^{-\imath \mathbf{q} \cdot \mathbf{r}} d \mathbf{r}\right|^{2}
$$

where $\Omega$ is the crystal volume. The $p$-Ps and $o$-Ps annihilation rates in a condensed medium, $\lambda_{1}$ and $\lambda_{3}$ respectively, can now be written as

$$
\lambda_{1}=\lambda_{1, \mathrm{sa}}+\lambda_{\mathrm{po}}, \quad \lambda_{3}=\lambda_{3, \mathrm{sa}}+\lambda_{\mathrm{po}}
$$

where $\lambda_{1, s a}$ and $\lambda_{3, s a}$ are the singlet and triplet self-annihilation rates and $\lambda_{\text {po }}$ is the pick-off annihilation rate. These rates are obtained from (3.9) using (2.42)

$$
\begin{aligned}
\lambda_{1, \mathrm{sa}} & =4 \pi r_{o}^{2} c \int_{V}\left|\Psi_{\mathrm{Ps}}(\mathbf{r}, \mathbf{r})\right|^{2} d \mathbf{r} \\
\lambda_{\mathrm{po}} & =\pi r_{o}^{2} c \sum_{\mathbf{k} j} \int_{V} d \mathbf{r}_{e} \int_{V}\left|\Psi_{\mathrm{Ps}}\left(\mathbf{r}_{e}, \mathbf{r}\right) \Psi_{\mathbf{k} j}(\mathbf{r})\right|^{2} d \mathbf{r} .
\end{aligned}
$$

The effects of a medium on Ps are often described by introducing two parameters $\kappa$ and $\eta . \kappa$ is called the internal contact density and is defined as

$$
\kappa=8 \pi a_{o}^{3} \int_{V}\left|\Psi_{\mathrm{Ps}}(\mathbf{r}, \mathbf{r})\right|^{2} d \mathbf{r}
$$

which is simply the overlap probability of the positron with its bound electron, normalized to the contact density of an isolated Ps atom. The external contact density

$$
\eta_{\mathbf{k j}}=8 \pi a_{\mathrm{o}}^{3} \int_{V} d \mathbf{r}_{e} \int_{V}\left|\Psi_{\mathrm{Ps}_{\mathrm{s}}}\left(\mathbf{r}_{e}, \mathbf{r}\right) \Psi_{\mathbf{k j}}\left(\mathbf{r}_{e}\right)\right|^{2} d \mathbf{r}
$$

represents the overlap probability of the positron with the $(\mathbf{k} j)^{\text {th }}$ electron. Using these definitions the Ps annihilation rates can be written as

$$
\begin{aligned}
& \lambda_{1}^{o}=\kappa \lambda_{1}^{\operatorname{vac}}+\lambda_{\mathrm{Ps}_{\mathbf{s}}}^{\operatorname{vac}} \sum_{\mathbf{k} j} \eta_{k j} \\
& \lambda_{3}^{o}=\kappa \lambda_{3}^{\mathrm{vac}}+\lambda_{\mathrm{Ps}_{\mathbf{s}}}^{\operatorname{vac}} \sum_{\mathbf{k} j} \eta_{k j}
\end{aligned}
$$


where $\lambda_{\mathrm{Ps}}^{\mathrm{vac}}, \lambda_{1}^{\mathrm{vac}}$ and $\lambda_{3}^{\mathrm{vac}}$ are given by (3.5), (3.7) and (3.8), respectively. For an isolated Ps atom $\kappa=1$ and $\eta_{i}=0$. In a condensed medium, the bound electron-positron pair is subject to external disturbances which reduce their overlap probability (i.e. $\kappa<1$ ). A small value of $\kappa$ implies a large $\lambda_{\text {po }}$, simply because the electron in Ps cannot follow the positron into regions occupied by other electrons in fully closed shells. The relationship between $\kappa$ and $\lambda_{\text {po }}$ depends on the structure of the system. A reduced internal contact density also implies a Ps hyperfine splitting $\Delta E_{\mathrm{Ps}_{1},}=E_{o-\mathrm{Ps}_{, 1}}-E_{\mathrm{p}-\mathrm{Ps}, 1}$ and binding energy $E_{\mathrm{Ps}, \mathrm{b}}$ that are smaller than the vacuum values. An electron-positron pair with a spherically symmetric spatial wave function has a hyperfine splitting that scales with the internal contact density

$$
\Delta E_{\mathrm{Ps}, 1}=\kappa \Delta E_{\mathrm{Ps}, 1}^{\mathrm{vac}}
$$

Distortions in the Ps wave function give contributions to $\Delta E_{\mathrm{Ps}, 1}$ not proportional to $\kappa$, however these contributions can usually be neglected. The detailed dependence of $\Delta E_{\mathrm{Ps}, \mathrm{b}}$ on $\kappa$ also depends on the system (Dupasquier 1988).

\subsection{Positronium Formation Models}

In molecular and insulating condensed media, some fraction of the injected positrons typically form a bound state with an electron that in many ways resembles the positronium atom described above. The processes governing the formation and yield of Ps in condensed matter are complicated and not yet properly understood. The process is usually described in terms of one of the two competing theories - the Ore gap model (Ore 1949) or the positron spur model (Mogensen 1974; Byakov et al. 1974). The Ore gap model was originally proposed to describe Ps formation in low density gases and has since been extended to condensed matter. Since radiative spurs do not form in gases, this model is generally considered the one most applicable in low-density gases. However, in condensed matter, where the positron can pick up a 'free' electron from the positron spur to form Ps, there remains some controversy as to whether the Ore gap or spur model is correct. Both models are described below. As will be seen, the simple materials used in this thesis provide some interesting insight into the applicability of these models.

It is generally accepted that the Ore gap model best describes Ps formation in gases where positron spurs cannot form. In this model, an energetic positron captures an electron 
during a collision to form Ps

$$
\left(\mathrm{e}^{+}\right)^{*}+\mathrm{M} \rightarrow \mathrm{Ps}^{*}+\mathrm{M}^{+} \rightarrow \mathrm{Ps}+\mathrm{M}^{+}
$$

where $\left(\mathrm{e}^{+}\right)^{*}$ is an epithermal positron with kinetic energy $E_{\mathrm{p}}, \mathrm{M}$ is a gas atom and $\mathrm{Ps}^{*}$ and Ps are epithermal and thermalized states of Ps respectively. For $E_{\mathrm{p}}$ above the first ionization energy $E_{\text {ion }}\left(=E_{u}\right)$ the Ps formation probability is small. The reason is that the resulting Ps would be formed with a kinetic energy greater than its binding energy and would likely break up again during subsequent collisions. The small Ps binding energy implies that (3.17) is usually endothermic and therefore also has a lower energy threshold $E_{l}=E_{u}-6.8 \mathrm{eV}$ below which Ps formation is impossible. Therefore, to form Ps via (3.17) the thermalizing positron must fall within the energy range $E_{l}<E_{\mathrm{p}}<E_{u}$ known as the 'Ore gap'. For positrons with energy above the lowest excitation energy $E_{\mathbf{x}}$ (i.e. in the energy range $E_{\mathrm{x}}<E_{\mathrm{p}}<E_{\mathrm{u}}$ ) Ps formation must compete with electronic excitations. For positrons falling into the energy range $E_{l}<E_{\mathrm{p}}<E_{\mathrm{x}}$ elastic scattering and Ps formation are the most important processes. In gases, positrons that fall below the Ore gap $\left(0<E_{\mathrm{p}}<E_{l}\right)$ annihilate as free particles.

After their last ionizing collision positrons fall between zero and $E_{u}$ with a distribution $g\left(E_{\mathrm{p}}\right) d E_{\mathrm{p}}$ such that $\int_{0}^{E_{x}} g(E) d E=1$. Starting from their initial 'touch down' energy, positrons falling within the Ore gap lose energy at some mean rate $-d E_{\mathrm{p}}(t) / d t=\gamma_{\mathrm{r}} E_{\mathrm{p}}(t)$ where $\gamma_{r}$ is the characteristic energy loss rate in the Ore gap. The Ps yield is approximately

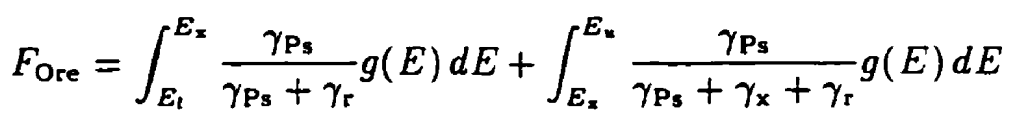

where $\gamma_{P_{s}}$ is the Ps formation rate in the Ore gap and $\gamma_{x}$ is the electronic excitation rate. Recall that these rates are related to their corresponding cross sections $\sigma$ by $\gamma=n v \sigma$ where $n$ is the density of scatters and $v$ is the positron velocity. In most approximations, the initial positron energy distribution is assumed to be uniform (i.e. $g\left(E_{\mathrm{p}}\right)=E_{u}^{-1}$ ) between zero and $E_{u}$. In the rare gases, elastic scattering is an inefficient energy loss process (i.e. $\gamma_{r} \ll \gamma_{P_{s}}$ ) and so positrons in the Ore gap are effectively hung up there until either an excitation, when possible, or Ps formation occurs. In molecular gases, $\gamma_{r}$ is generally larger due to the additional thermalization modes present. Clearly, the larger $\gamma_{P_{s}}$ is in relation to $\gamma_{x}$ and $\gamma_{r}$ the larger the Ps yield. Upper and lower boundaries on the Ore gap Ps yield are often calculated assuming $\gamma_{r}, \gamma_{x} \ll \gamma_{P_{s}}$ and $\gamma_{r} \ll \gamma_{P_{s}} \ll \gamma_{x}$, respectively. Doing this gives $\left(E_{\mathrm{x}}-E_{l}\right) / E_{\mathrm{u}}<F_{\text {Ore }}<6.8 \mathrm{eV} / E_{\mathrm{u}}$. The Ore gap estimates and the observed Ps yields for the rare and diatomic gases can be found in Tables A.I and A.2, respectively. 
These same ideas can now be applied to condensed matter with little modification. For wide band-gap insulators the upper Ore gap boundary is approximately $E_{u}=E_{\text {gap }}$, where $E_{\text {gap }}$ is the band-gap energy. The lower Ore gap boundary is $E_{l}=E_{u}-E_{\mathrm{Ps}, \mathrm{b}}$, where $E_{\mathrm{Ps}, \mathrm{b}}$ is the Ps binding energy within the material. Equation (3.18) may again be used to estimate the Ore gap Ps yield. Using the same approximations as before, one can easily obtain upper and lower estimates of this yield

$$
\frac{E_{\mathrm{Ps}, \mathrm{b}}-E_{\mathrm{x}, \mathrm{b}}}{E_{\mathrm{u}}}<F_{\mathrm{Ore}}<\frac{E_{\mathrm{Ps}, \mathrm{b}}}{E_{u}}
$$

where $E_{\mathbf{x}, \mathrm{b}}=E_{\mathrm{gap}}-E_{\mathbf{x}}$. In the rare-gas solids this is the exciton binding energy. In general, $E_{\mathrm{Ps}_{\mathrm{b}} \mathrm{b}}$ is unknown making it difficult to estimate the Ps formation fraction. Fortunately, a series of important slow positron experiments have measured some of these quantities in the heavier rare-gas solids and in solid $N_{2}$. A summary of these results can be found in Table 6.3.

If a positron bypasses the Ore gap, or scatters out of it without forming Ps, then it may still form Ps by capturing an electron from the conduction band. Recall from $§ 2.3 .3$ that when an energetic positron enters a material it generates an ionization trail. In that last part of this trail, the positron's motion is diffusive so that once the positron thermalizes it is surrounded by ionization products - excited atoms, ions and ionized electrons, etc.which together form the positron spur. The positron and ionization products are generally close enough together that there is a significant probability of their interacting prior to the eventual collapse of the spur. In the spur model, Ps formation is usually assumed to occur between a thermalized positron and a spur electron

$$
\left\{\left(\mathrm{e}^{+}\right)^{-}+\mathrm{M}\right\} \rightarrow\left\{\mathrm{e}^{+}+\mathrm{e}_{s}^{-}+\mathrm{M}^{+}\right\} \rightarrow\left\{\mathrm{Ps}+\mathrm{M}^{+}\right\}
$$

where \{\} denote reactions occurring inside the positron spur. The only energy requirement on (3.20) is that $E_{\mathrm{Ps}, \mathrm{b}}>E_{\mathrm{Ae}}+E_{\mathrm{Ap}}$, where $E_{\mathrm{Ae}}$ and $E_{\mathrm{Ap}}$ are the electron and positron affinities of the atoms in the medium. The efficiency of the spur formation mechanism is controlled by several factors: the number and distribution of species in the spur (i.e. the 
initial distance between $\mathrm{e}^{+}$and $\mathrm{e}_{s}^{-}$) and a number of other possible spur reactions

$$
\begin{aligned}
&\left\{\mathrm{e}_{s}^{-}+\mathrm{M}^{+}\right.\left.\rightarrow \mathrm{M}^{-} \rightarrow \mathrm{M}\right\} \\
&\left\{\mathrm{e}^{+}, \mathrm{e}_{s}^{-}, \mathrm{M}^{+}\right\} \rightarrow \mathrm{e}^{+}, \mathrm{e}_{s}^{-}, \mathrm{M}^{+} \\
&\left\{\mathrm{e}^{+}+\mathrm{M}\right.\left.\rightarrow 2 \gamma+\mathrm{M}^{+}\right\} \\
&\left\{\mathrm{e}^{+}+\mathrm{M}^{*}\right.\left.\rightarrow \mathrm{Ps}+\mathrm{M}^{+}\right\} \\
&\left\{\mathrm{Ps}+\mathrm{M}^{+} \rightarrow \mathrm{e}^{+}+\mathrm{M}\right\} \\
&\left\{\mathrm{e}_{s}^{-}+\mathrm{Ps} \rightarrow \mathrm{Ps}^{-}\right\}
\end{aligned}
$$

The spur Ps formation fraction depends largely on the relative strength of (3.20) as compared to $(3.21 \mathrm{a}, \mathrm{b}, \mathrm{c})$, where (3.21a) describes both germinate and random recombination of spur electrons and positive ions, (3.21b) describes the diffusion of the positron and/or secondary electrons out of the spur and (3.21c) is the annihilation of free positrons. Reactions (3.21d,e,f) are of secondary importance.

Much of the experimental support for the spur model has come from measuring the Ps yield as a function of the concentration of some electron acceptor or donor added to the liquid sample. For a strong electron acceptor $\mathrm{A}$, the reactions

$$
\begin{gathered}
\left\{e_{s}^{-}+A_{s} \rightarrow A_{s}^{-}\right\} \\
\left\{e^{+}+A_{s}^{-} \rightarrow e^{+} A_{s}^{-}\right\}
\end{gathered}
$$

compete with (3.20) and inhibit Ps formation. Weak electron acceptors $A_{w}$ often have an 'anti-inhibition' effect

$$
\begin{aligned}
& \left\{\mathrm{e}_{s}^{-}+\mathrm{A}_{w} \rightarrow \mathrm{A}_{w}^{-}\right\} \\
& \left\{\mathrm{e}^{+}+\mathrm{A}_{w}^{-} \rightarrow \mathrm{Ps}_{\mathrm{s}}+\mathrm{A}_{w}\right\}
\end{aligned}
$$

A summary of the effects of various inhibitors can be found in Ito (1982). The obvious complexity of the positron spur makes it difficult to calculate a meaningful estimate of the spur Ps formation fraction.

At present, a quantitative assessment of the relative importance of the two Ps formation mechanisms is difficult. Experiments in gaseous $\mathrm{He}, \mathrm{Ar}, \mathrm{H}_{2}$ and $\mathrm{CH}_{4}$ confirm that Ps formation occurs mainly in the Ore gap energy range and that the Ps has an initial kinetic energy of a few eV (Charlton et al. 1980). There is also evidence for combined Ore gap and spur Ps formation in several dense polyatomic molecular gases (Heyland et al. 1985). 
Several monoenergetic positron beam experiments have provided evidence of the validity of the Ore gap mechanism in wide band-gap insulators. Experiments in crystalline ice (EIdrup et al. 1983,1985) have measured Ps yields as a function of the incident positron energy. The results are well described by the Ore gap model for incident positron energies that are too low for an effective positron spur to form. At higher energies (i.e. $>100 \mathrm{eV}$ ) the Ps yield increases with energy due to spur formation. Similar experiments have shown that the Ore gap process is an important mechanism in the rare-gas solids (Gullikson and Mills 1986,1988,1989, 1990; Mills and Gullikson 1986). Spur Ps formation was not seen in these experiments as the incident positron energies were too low to generate an effective positron spur. Tuomisaari et al. (1989, 1991) have measured the energy distributions of positronium emitted from the heavier rare-gas solids and the alkali halides following the implantation of monoenergetic positrons with energies ranging from 0.1 to several $\mathrm{keV}$. These studies suggested that in all of these materials the Ore gap was the dominant formation process, except for LiF which also appears to have a spur component. Experiments measuring the Ps yield as a function of a static electric field in several molecular plastics provides further evidence for Ps formation through both the Ore gap and spur processes ${ }^{\dagger}$ (Bisi et al.1983). Similar experiments have recently shown that the spur formation process is important in liquid $\mathrm{N}_{2}$ (Pepe et al. 1993).

In many other molecular liquids and solids, there exists a huge body of experimental evidence that appears to be explained, at least qualitatively, by the positron spur model. Many of these results cannot be explained by the Ore gap model even when it is modified to allow various other processes ${ }^{\ddagger}$. However, it is important to understand that the two formation models are not mutually exclusive, despite what some of the proponents in the Ps formation controversy contend. The Ore gap mechanism precedes spur formation in time. When both formation processes are possible it is likely that Ore gap formation will be dominant. However, for polyatomic molecules it is possible to have inelastic scattering thresholds that lie below the lower Ore gap boundary. In this case, thermalizing positrons no longer become hung up in the Ore gap and the spur model dominates. The relative fraction of Ore gap to spur formation determines the sensitivity of the Ps yields to various experiments.

\footnotetext{
$\dagger_{\text {Mogensen }}(1983,1986)$ and others have criticized many of these conclusions and have presented arguments describing the results in terms of the spur model alone.

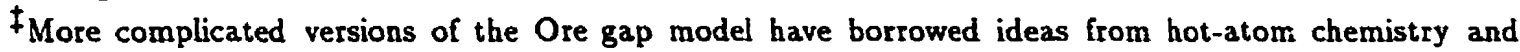
have described Ps inhibition by allowing processes such as the capture of positrons above the lower Ore gap boundary, the rapid thermalization of positrons through the Ore gap, and reactions with epithermal Ps.
} 
Finally, it is important to mention that in solids there may simply not be enough "free volume' in the lattice for Ps to survive. Like the positron, the Ps wave function amplitude is small in the atomic core regions due to the electrostatic repulsion and exchange forces between its electron and the core electron distribution surrounding each nucleus. For both positrons and Ps, this repulsion is partially counterbalanced by polarization forces. Bloch state Ps also has a 'Swiss cheese' character and is said to exist in the free volume outside of an 'excluded volume' around each atom. Brandt et al. $(1960,1966,1970)$ have developed a model in which the Ps is assumed to reside in the free volume between atoms without altering the structure of the medium and have had some success describing changes in the positron and o-Ps lifetimes as a function of temperature and density. The curvature of the thermalized Ps Bloch-state wave function, and therefore its ground state energy, is determined by the amount of available free volume. If the available free volume is too low then the Ps ground-state energy will exceed its binding energy and will likely break up again in subsequent collisions. As will be seen, it is the lack of free volume in the rare-gas solids that results in Ps yields that are significantly lower than in the liquids.

\subsection{Positronium States in Condensed Matter}

Once Ps forms, it has an interesting and often complicated history, even in the simplest of condensed media. Like the positron, Ps will thermalize and exist in one or more various states from which it eventually annihilates. The competition between these states is determined by the interactions between the light particle and the medium. In condensed media with sufficient free volume it may be energetically favourable for Ps to exist in a delocalized state. In solids, the lack of free volume and/or energy considerations often cause Ps to localize at defect sites. In most liquids, the free energy of the system is minimized when Ps self-traps (i.e. creates its own free volume) into a 'bubble' state. The possibility of $\mathrm{Ps}$ bound states with atoms and/or molecules in a medium or $\mathrm{Ps}^{-}$ion formation must also be considered. In what follows, each of these possibilities is discussed. Relevant physical processes and chemical reactions that also affect Ps formation and its annihilation characteristics are discussed in $\$ 3.5$.

The thermalization process for delocalized Ps is similar to that for a positron near the end of its ionization trail. Ps formed in the Ore gap is expected to have an initial kinetic energy between zero and its binding energy. Spur Ps starts out with at least $E_{\mathrm{Ps}, \mathrm{b}}$. 
In either case, Ps thermalizes very quickly either through acoustic phonon scattering in solids or density fluctuation scattering in liquids. The deformation potential calculations in Appendix $C$, coupled with other experimental results mentioned there, suggest that in the condensed rare gases Ps comes to thermal equilibrium within 10 ps. The thermalization times in the condensed diatomic gases are expected to be somewhat faster due to the additional thermalization processes available to molecular media.

\subsubsection{Delocalized Positronium States}

Bloch state Ps has been observed in single crystal alkali halides, quartz (Brandt et al. 1969; Hodges et al. 1972; Berko et al. 1977), ice (Eldrup et al. 1983,1985) and several other solids. These delocalized states are easily distinguished from other states due to the presence of a relatively narrow peak in the momentum density distribution at $q=0$ and the presence of spectacular satellite peaks centred at momenta corresponding to the reciprocal lattice vectors. If the Ps wave function in (3.9) is replaced by a Bloch wave function of the form given by (2.5) (i.e. $\Psi_{\mathrm{Ps}_{1} \mathbf{k}}\left(\mathbf{r}_{\mathbf{e}}, \mathbf{r}_{\mathbf{p}}\right)=\exp (\mathrm{ik} \cdot \mathbf{R}) u_{\mathbf{k}}(\mathbf{R}, \varrho)$ where $\mathbf{R}$ and $\varrho$ are defined as in $§ 3.1)$ then the $2 \gamma$ self-annihilation momentum density becomes a discrete spectrum of the form

$$
\rho_{1, \text { sa }}^{\prime}(\mathbf{q})=\frac{4 \pi r_{0}^{2} c}{8 \pi^{3}} \sum_{\mathbf{G}}\left|\frac{\delta(\mathbf{q}-\mathbf{k}-\mathbf{G})}{\Omega_{0}} \int_{\Omega_{0}} u_{\mathbf{k}}(\mathbf{R}, 0) e^{-i \mathbf{G} \cdot \mathbf{r}} d \mathbf{R}\right|^{2}
$$

where $\Omega_{0}$ is the volume of the Wigner-Seitz cell. The spectrum consists of lines centred at $\mathbf{q}=\mathbf{k}+\mathbf{G}$ each having a relative weight given by (3.26), which gives satellite peaks smaller than the central one. The ensemble of delocalized Ps is generally assumed to have a Boltzmann energy distribution which causes a broadening of the lines given by (3.26). The peaks therefore become Gaussian shaped with a full width at half maximum of $\sqrt{8 \ln (2) m_{\mathrm{Ps}_{\mathrm{s}}}^{*} k_{\mathrm{B}} T}$ where $m_{\mathrm{Ps}_{\mathrm{s}}}^{*}$ is the Ps effective mass. Imperfections in the crystal have also been observed to scatter the delocalized Ps state causing a further broadening of the peak widths.

\subsubsection{The Positronium Bubble State}

Light quantum particles immersed in a condensed medium exist in either delocalized or localized states. Ps is generally formed, and then thermalizes, in a delocalized state hav. ing a deBroglie wavelength many times greater than the average separation between atoms. 
Due to the strong Pauli exchange repulsion between the electron in Ps and the electrons of parallel spin in the surrounding medium Ps tends to be repelled from the atoms into the free volume surrounding them. Regions of lower than average density - e.g. vacancies and vacancy clusters in solids and density fluctuation in liquids - are seen as attractive potential wells. Thus, in solids the Ps wave function may collapse and become localized at a defect if it provides a large enough potential well. In liquids, one imagines that the Ps wave function amplitude is largest in density fluctuations where the electron density is lowest and that the Ps wave function is easily able to follow the density fluctuations. As the Ps reaches complete thermalization, it can further minimize the exchange repulsion by collapsing its wave function into one of the density fluctuations and then pushing the medium away to create a cavity, or bubble, in the liquid. Unlike solids, the structural rearrangements required for self-trapping in liquids can occur on a time scale that is short compared to the Ps lifetime. In general, these self-trapped states will be preferred thermodynamically if the change in free energy of the system $\Delta F$ on going from a delocalized to localized state is negative

$$
\Delta F=E_{2 p}+E_{b}-E_{o}<0
$$

where $E_{z p}$ is the Ps zero-point energy in the localized state, $E_{b}$ is the energy necessary to form the bubble and $E_{o}$ is the energy at the bottom of the Ps conduction band, which can also be calculated using the techniques described in $\$ 2.4 .1$. Localized bubble states will be formed if the binding energy to the bubble $\left(E_{o}-E_{z p}\right)$ is greater than the work done to create it. Transitions to the bubble state occur as the density rises or the temperature drops - either factor intensifies the Ps-atom interaction. Therefore, at low enough gas and fluid densities, the delocalized plane-wave state is stable, while at higher fluid densities the localized state generally becomes the most probable.

The phenomenon of Ps self-trapping in liquids was first observed by Paul and Graham (1957) and Wackerle and Stump (1957). They measured lifetime spectra in liquid helium and unexpectedly found an $o$-Ps component whose annihilation rate was comparable to its vacuum value and not the expected pick-off rate. The explanation was provided by Ferrell (1957) who suggested that inside a bubble state the Ps lifetime was increased due to a reduced overlap with the electrons in the medium. The existence of Ps bubbles was beautifully demonstrated by Briscoe et al. $(1967,1968)$ and Stewart and Briscoe $(1967)$ who made the first quantitative measurements of the bubble parameters by measuring the $2 \gamma$ momentum densities in liquid $\mathrm{He}, \mathrm{Ne}, \mathrm{Ar}, \mathrm{H}_{2}, \mathrm{~N}_{2}$ and $\mathrm{O}_{2}$. They found a narrow component 
in the momentum density due to the self-annihilation of $p$-Ps which corresponded nicely with the zero-point energy and momentum for a Ps trapped in a spherical potential well. Triftshauser et al. (1969) extended this work by measuring the pressure dependence of the bubble radius in both liquid and solid helium and provided further convincing evidence in support of the bubble state. Since this initial work there have been several angular correlation and numerous lifetime measurements in the condensed rare and diatomic gases. All of the known experimental results relevant to this work are summarized in Tables 4.1 and 4.3. The Ps bubble state has now been observed and studied extensively in a wide range of low viscosity liquids.

The simplest theoretical model that can be used to describe the equilibrium bubble state is known as the square-well model (Ferrell 1957; Roellig and Kelly 1967; Tao 1972; Beling and Smith 1980). In this model, the Ps is assumed to be trapped in a spherical cavity of radius $R$. In the simplest approximation, the density profile of the medium around the Ps is assumed a priori to be a step function - zero inside the bubble and $n_{o}$ outside. The interaction with the medium is represented by a spherically symmetric potential barrier

$$
V(r)= \begin{cases}0 & r<R_{1} \\ E_{0} & r>R_{1}\end{cases}
$$

where the Ps is treated as a hard sphere with a radius equal to its scattering length $a_{s}$ so that $R_{\mathrm{l}}=R-a_{s}$.

The outward pressure required to sustain the bubble state is provided by centreof-mass zero-point motion of the localized Ps. The solution to the Schrödinger equation for the ground-state centre-of-mass motion of the Ps is identical to that for a particle in a spherical square-wall potential which is described in many introductory quantum mechanics texts. The bound ground-state Ps wave function (i.e. $l=0$ ) is spherically symmetric and has the form

$$
\Psi_{\mathrm{Ps}_{\mathrm{s}}}(\mathrm{r})= \begin{cases}A \sin (\alpha r) / r & r<R_{1} \\ B \mathrm{e}^{-\beta r} / r & r>R_{1}\end{cases}
$$

where the constants $A$ and $B$ are determined from the usual normalization conditions and the continuity of the logarithmic derivative at $r=R_{1}$

$$
A=\sqrt{\frac{\beta}{2 \pi\left(\beta R_{1}+1\right)}}, \quad B=A \mathrm{e}^{\beta R_{1}} \sin \left(\alpha R_{1}\right) .
$$

The wave numbers are given by

$$
\alpha^{2}=\frac{2 m_{\mathrm{P}_{s}}^{*}}{\hbar^{2}} E_{z p}, \quad \beta^{2}=\frac{2 m_{\mathrm{P}_{s}}^{*}}{\hbar^{2}}\left(E_{o}-E_{z p}\right)
$$


and are related by

$$
\beta=-\alpha \cot \left(\alpha R_{\mathrm{I}}\right)
$$

Using these results the zero-point energy may be written as

$$
E_{z p}=E_{o} \sin ^{2}\left(\alpha R_{1}\right)
$$

with $\frac{\pi}{2}<\alpha R_{1}<\pi$. Equation (3.33) is transcendental and so the value of $\alpha R_{1}$ must be determined either graphically or numerically. It is easily shown that no bound state exists unless $E_{o} R_{1}^{2}>(\pi \hbar)^{2} /\left(8 m_{\mathrm{P}_{\mathrm{s}}}^{*}\right)$.

As a first approximation, the energy required to rearrange a medium to form a bubble is

$$
E_{b}=\frac{4}{3} \pi R^{3} P+4 \pi R^{2} \sigma
$$

where $\sigma$ is the specific surface energy, which in liquids is more commonly called the surface tension. The first term in (3.34) corresponds to the work required to remove atoms from within the bubble volume against the liquid pressure $P$. The second term corresponds to the work required to form and maintain a surface in the liquid. The experimental surface tension along a liquid-vapour interface provides only a lower limit on the estimate of work required to form a surface in a microscopic bubble. However, the effects arising from the curvature of the bubble surface have been shown to be small (Padmore and Cole 1974; Smith et al. 1977; Beling and Smith 1980). Combining (3.33) and (3.34) the total energy of the bubble state may be written as

$$
E_{\mathrm{bub}}=E_{z \mathrm{p}}+E_{b}=\frac{\hbar^{2} \alpha^{2}}{2 m_{\mathrm{Ps}}^{2}}+\frac{4}{3} \pi R^{3} P+4 \pi R^{2} \sigma
$$

For an infinite potential well $\left(E_{o} \rightarrow \infty\right) \alpha=\pi / R_{1}$.

Using the above results it is possible to obtain estimates for the bubble radius, the pick-off annihilation rate and the momentum density. The equilibrium bubble radius is determined by minimizing (3.35) with respect to $R$. For the special case of an infinite potential well it is possible to obtain an analytic expression for the equilibrium radius

$$
\begin{gathered}
\frac{\partial E_{\mathrm{bub}}}{\partial R}=0=-\frac{\hbar^{2} \pi^{2}}{m_{\mathrm{P}_{\mathrm{s}}}^{*} R_{1}^{3}}+8 \pi R \sigma+4 \pi R^{2} P \\
R_{1}^{3} R=\frac{\hbar^{2} \pi}{4 m_{\mathrm{P}_{\mathrm{s}}}^{*}(2 \sigma+R P)} .
\end{gathered}
$$


For a finite potential, the radius must be solved for using an iterative procedure to minimize (3.35), with (3.36) being used as an upper limit. In the rare gas liquids, $\sigma$ increases with $Z$ and the polarizability. This implies a large bubble in relatively unpolarizable liquid helium and an increasingly smaller bubble in the heavier rare gas liquids.

The annihilation rate from the bubble state is usually written as

$$
\lambda_{1,3}^{o}=\kappa \lambda_{1,3}^{\mathrm{vac}}+P \lambda_{\mathrm{po}}
$$

where $P$ is the probability that the Ps centre-of-mass is outside the bubble. As a first approximation it can be written as

$$
\begin{aligned}
P & =4 \pi \int_{R_{1}}^{\infty}\left|\Psi_{\mathrm{Ps}_{s}}(r)\right|^{2} r^{2} d r \\
& =\frac{\alpha^{2}}{\left(\alpha^{2}+\beta^{2}\right)\left(\beta R_{1}+1\right)}
\end{aligned}
$$

where it has been assumed that the overlap for the positron is the same as that for the Ps - i.e. zero Ps polarization. In the bubble state, it is expected that $\kappa>0.9$ since the overlap with the medium is significantly reduced. Instead of using ( $3.11 \mathrm{~b})$ to calculate the bulk pick-off annihilation rate, it is usually written as

$$
\lambda_{\mathrm{po}}=4 \pi r_{0}^{2} c n^{1} Z_{\mathrm{eft}}
$$

where ${ }^{1} Z_{\text {eff }}$ is the effective number of electrons per atom available for pick-off annihilation with $o$-Ps. The factor of four arises from the fact that only $\frac{1}{4}$ of the atomic electrons are in a singlet state relative to the positron in $o-\mathrm{Ps}^{\dagger}$. Since $o$-Ps in the bubble state annihilates mostly with one of the outer valence electrons, $4^{1} Z_{\text {eff }}$ is usually taken to be the sum of the valence electrons (or ' $Z_{\text {eff }}$ is the sum of the valence electrons that are singlet with respect to the positron).

The momentum density for $2 \gamma p$-Ps self-annihilation from the bubble state can be calculated using (3.9a). For the special case of an infinite spherical square potential well it is easily shown that

$$
\rho_{\infty}(\mathbf{q})=\frac{4 \pi r_{o}^{2} c}{8 \pi^{3}}\left(2 \pi R_{1}\right)^{3}\left(\frac{\sin \xi}{\xi\left(\pi^{2}-\beta^{2}\right)}\right)^{2}
$$

\footnotetext{
${ }^{\dagger}$ Care should be taken not to confuse $4^{1} Z_{\text {eff }}$ with $Z_{\text {eff }}$, which is the effective number of electrons available for free positron annihilation, as has been done in the past.
} 
where $\xi=q R_{1}$. For future reference, this momentum density gives rise to a component in the one-dimensional angular correlation curves of the form (see $§ 4.1$ )

$$
N_{\propto}\left(p_{z}\right)=K\left(\pi r_{0}^{2} c\right)\left(8 \pi \hbar^{2} R_{1}\right) \int_{p_{s} R_{1} / \hbar}^{\infty}\left(\frac{\sin \xi}{\xi\left(\pi^{2}-\beta^{2}\right)}\right)^{2} \frac{d \xi}{\xi}
$$

where $p_{z}=\hbar q_{z}$ and $K$ is a constant which accounts for the arbitrary normalization of experimental angular correlation curves (i.e. the constants in brackets are not important). It is a simple matter to integrate (3.42) to find the value of the lower integration limit that corresponds to the half height of $N_{\infty}\left(p_{z}\right)$. Doing this gives a simple estimate of the bubble radius from the full width at half maximum of $N_{\infty}\left(p_{z}\right)$

$$
R_{1} \theta_{n}=16.59 \AA \text { mrads }
$$

For the finite potential well, inserting (3.29) into (3.9a) gives a momentum density

$$
\rho_{o}(\mathbf{q})=\frac{4 \pi r_{o}^{2} c}{8 \pi^{3}}\left(4 \pi A R_{1}\right)^{2}\left(\alpha_{1}^{2}+\beta_{1}^{2}\right) \alpha_{1}^{2}\left(\frac{\xi \cos \xi+\beta_{1} \sin \xi}{\xi\left(\alpha_{1}^{2}-\xi^{2}\right)\left(\beta_{1}^{2}+\xi^{2}\right)}\right)^{2}
$$

where $\alpha_{1}=\alpha R_{1}$ and $\beta_{1}=\beta R_{1}$. The corresponding component in the angular correlation curve is

$$
N_{o}\left(p_{z}\right)=K\left(\pi r_{0}^{2} c\right)\left(4 \hbar R_{1} A\right)^{2}\left(\alpha_{1}^{2}+\beta_{1}^{2}\right) \alpha_{1} \int_{p_{z} R_{1} / \hbar}^{\infty}\left(\frac{\xi \cos \xi+\beta_{1} \sin \xi}{\left(\alpha_{1}^{2}-\xi^{2}\right)\left(\beta_{1}^{2}+\xi^{2}\right)}\right)^{2} \frac{d \xi}{\xi}
$$

where $A$ is given by (3.30). Using a three dimensional harmonic potential, instead of a spherical square-well potential, makes both $\rho(q)$ and $N\left(p_{z}\right)$ Gaussian shaped and gives a significantly smaller estimate for the bubble radius

$$
R_{1} \theta_{n}=10.7 \quad \AA \text { mrads }
$$

The equilibrium bubble state has also been described using several far more sophisticated approaches (see the reviews by lakubov and Khrapak 1982 and Hernandez 1991 and references therein). The goal of most of these studies has been to determine the temperature and density range in which self-trapping is possible. Sharma et al. (1982b) have developed a model which treats $\mathrm{Ps}$ as a passive probe of the spontaneous density fluctuations and ignores the interactions between $P$ s and the medium. The model is successful in describing the transition region between delocalized and localized states but it has serious difficulties once strongly bound localized states become predominant. Stott and Zaremba (1977) developed a density-functional approach to describe the formation of positron self-trapped states in helium droplets. Nieminen et al. (1980) extended this approach to self-trapped Ps 
bubbles. In their work, the free-energy density functional is minimized and the seif-trapped Ps wave function and the density profile about the bubble are determined self-consistently. This approach has been used to study the bubble state in helium (Nieminen et al. 1980; Rytsölä et al. 1984b), argon (Tuomisaari et al. 1986), xenon (Tuomisaari et al. 1988) and $\mathrm{N}_{2}$ (Rytsölä et al. 1984a). The theory neglects the effects of density fluctuations and is therefore unable to properly describe the transition region or the dynamics of bubble formation. However, it has achieved a good qualitative agreement with experimental results in dense gases and fluids. At higher liquid densities, comparable to those used in this work, these calculations show that the thickness of the boundary layer between the bubble and the medium is very small and that a step function density profile is a reasonable first approximation. Considering the quality of our experimental data and the large Ps binding energy to the bubble, the square-wall potential should be more than adequate to describe the equilibrium bubble properties. In general, it is expected that both delocalized and localized states are populated and should be considered, especially when trying to describe the transition region at lower gas and fluid densities. However, at the higher liquid densities the bubble binding energy is large compared to $k_{B} T$ which implies that only the bubble state needs to be considered - i.e. the probability of delocalized states being occupied is negligible.

Before leaving the topic of Ps self-trapping it is useful to mention that excess electrons in liquid helium, neon and $\mathrm{H}_{2}$ are also thought to exist in bubble states (Levine and Saunders 1967; Harrison and Springett 1971a,1971b; Loveland et al. 1972; Bruschi et al. 1972; LeComber et al. 1976; Sakai et al. 1982; Borghesani and Santini 1990). Ps and electron bubble states have also been observed in solid helium which, unlike the other rare-gas solids, is very deformable ('Triftshäuser et al. 1969; Cohen and Jortner 1969). Delocalized electron states are favoured energetically in the heavier rare-gas solids. For neon the energy differences between a delocalized and an electron bubble state are small (Emery and Song 1986). Evidence for the electron bubble state is based on mobility measurements. For example, Levine and Saunders (1967) observed a decrease of $10^{3}$ to $10^{4}$ in electron mobility over a narrow density range on going from low to high density helium gas. The obvious interpretation is that at low densities the electron is delocalized, while at higher densities it is in a self-trapped bubble. The high-density mobilities are well described by the model of a charged sphere moving through a viscous medium (Stoke's Law) with kinetic-theory corrections. The electron mobilities in liquid helium, neon and $\mathrm{H}_{2}$ are $0.020,0.0016$ and 
$0.017 \mathrm{~cm}^{2} / \mathrm{Vs}$, respectively (see Appendix A). These are in contrast to the mobilities in liquid argon, krypton and xenon of 475,1900 and $2200 \mathrm{~cm}^{2} / \mathrm{Vs}$, respectively. Unlike Ps, the excess electrons in these liquids skitter along the bottom of the conduction band relatively unscattered - in fact, the heavier rare gases are among the weakest scattering liquids known. The difference between Ps and electrons arises from their interactions. Both particles feel the short-range Pauli exchange repulsion. Ps has a relatively weak van der Waals type polarization interaction. The short-range repulsion therefore dominates - i.e. Ps has a positive scattering length on atoms - and self-trapping occurs. However, for electrons, the polarization interaction is small only for helium and $\mathrm{H}_{2}$, and becomes comparable to the exchange interaction for neon. In the heavier rare gas liquids, the free energy is minimized when the atoms simply polarize in response to the excess electron. The resulting electron $s$-wave scattering length is negative - i.e. the interaction is attractive - and so no self-trapping occurs.

Many studies have also detected slow negative ions in the heavier rare-gas liquids. It is now accepted that these are probably $\mathrm{O}_{2}^{-}$impurity ions since ions such as $\mathrm{He}_{n}^{-}, \mathrm{Ar}_{n}^{-}$etc. are unstable, if they are formed at all (Nicolaides and Aspromallis 1991). Swan (1963,1964) and Dey and Lewis (1968) have suggested that oxygen concentrations as low as 1 p.p.m. in liquid Ar can trap many of the electrons to form $\mathrm{O}_{2}^{-}$with greatly reduced mobility. It is also now generally accepted that the low mobility positive charge carriers in the rare gas liquids are the diatomic ions $\mathrm{He}_{2}^{+}, \mathrm{Ar}_{2}^{+}$etc.

It is also important to know that the low electron mobility in liquid $\mathrm{O}_{2}$ and liquid $\mathrm{N}_{2}\left(0.0027 \mathrm{~cm}^{2} / \mathrm{Vs}\right)$ is most likely not due to self-trapping in a bubble state. It has long been known that low energy electrons (i.e. $<1 \mathrm{eV}$ ) can attach to oxygen molecules in the gas phase (Barabash et al. 1982; Hegerberg and Crompton 1983; Bruschi et al. 1984). The process is usually described in terms of the so-called Bloch-Bradbury two-stage process

$$
\begin{aligned}
\mathrm{e}^{-}+\mathrm{O}_{2}\left(\mathrm{X}^{3} \Sigma_{g}^{-}\right) & \rightleftharpoons \mathrm{O}_{2}^{--}\left({ }^{2} \Pi_{g} \text { or }{ }^{2} \Pi_{u}\right) \\
\mathrm{O}_{2}^{-\cdot}+\mathrm{M} & \rightarrow \mathrm{O}_{2}^{-}+\mathrm{M}
\end{aligned}
$$

where $\mathrm{O}_{2}^{-*}$ is an unstable negative ion in a vibrationally excited state $(v \geq 4)$ which either autoionizes within $10^{-12}-10^{-10} \mathrm{~s}$ or is stabilized by collisions with a third body $M$ to a vibrational level $(v<4)$ where autoionization becomes impossible ${ }^{\dagger}$. The energy of the first accessible vibrational level is $E_{4}=0.091 \mathrm{eV}$. The $\mathrm{O}_{2}$ electron affinity is $0.440 \mathrm{eV}$. While

\footnotetext{
${ }^{t_{\text {Several }}}$ studies in $\mathrm{O}_{2}-\mathrm{M}$ mixtures at higher pressures have also suggested that attachment to van der
} 
the effectiveness of the Bloch-Bradbury mechanism in liquids is unclear, one supposes that similar attachment reactions in liquid $\mathrm{O}_{2}$ results in small zero-field electron mobilities. In addition to attachment, or instead of it, many spur electrons in liquid $\mathrm{O}_{2}$ are thermalized so quickly that they simply cannot escape the Coulomb field of their parent ions.

In liquid $\mathrm{N}_{2}$, those electrons escaping recombination are thought to form a temporary anion state according to (3.47) and are partially stabilized though collisions with other $\mathrm{N}_{2}$ molecules and dimers (Wada and Freeman 1980; Gee et al. 1985). The anion state in liquid $\mathrm{N}_{2}$ is thought to be unstable with an effective lifetime on the order of picoseconds.

Finally, the existence of electron bubble states raises the interesting possibility that a thermalizing positron could find an electron in a bubble to form Ps in liquid helium, neon and $\mathrm{H}_{2}$. If this occurs, the initial release of the Ps binding energy would likely cause the Ps to pop out of the bubble (i.e. $E_{\mathrm{Ps}, b}>E_{o}-E_{z p}$ ), which would then collapse. After thermalizing in a delocalized state, the Ps could then self-trap in a new bubble. Thus, even though the spur electrons in these liquids may be trapped in bubbles, they could still affect the Ps formation fraction.

\subsubsection{Trapping at Defects in Solids}

The presence of lattice defects in an insulating solid can significantly affect the Ps yield. Simply cooling a rare-gas liquid to form a solid sample without a great deal of care produces a solid with a relatively large fraction of defect sites such as vacancies, voids (clusters of vacancies), dislocations, grain boundaries, etc. (Venables and Smith 1977). The electrostatic and exchange repulsion felt by the Ps cause it to see these regions of lower than average electron density as attractive potential wells. As with the positron, if these wells are large enough, it is possible for Ps to become bound in a localized state at the defect. For solids in which Bloch Ps states do exist, it may be energetically favourable for Ps to trap

Waals molecules containing $\mathrm{O}_{2}$ may also be important (Shimamori et al. 1981,1983,1984)

$$
\begin{aligned}
\mathrm{O}_{2}+\mathrm{M} & =\left(\mathrm{O}_{2} \cdot \mathrm{M}\right) \\
\mathrm{e}^{-}+\left(\mathrm{O}_{2} \cdot \mathrm{M}\right) & \rightarrow\left(\mathrm{O}_{2} \cdot \mathrm{M}\right)^{-\bullet} \\
\left(\mathrm{O}_{2} \cdot \mathrm{M}\right)^{-\bullet}+\mathrm{M} & \rightarrow \mathrm{O}_{2}^{-}+2 \mathrm{M}
\end{aligned}
$$

or simply

$$
\mathrm{e}^{-}+\left(\mathrm{O}_{2} \cdot \mathrm{M}\right) \rightarrow \mathrm{O}_{2}^{-}+\mathrm{M}
$$


at defect sites. In solids with a free volume that is too low to sustain thermalized Ps Bloch states, these lattice defects may offer the free volume required for Ps formation. If positrons also trap at the defects then it is possible for spur electrons, for example, to find them there and form Ps inside the defect. The difficulty with this, however, is that the localized Ps state must now dissipate the excess Ps binding energy, which is likely to be higher in the defect than in the bulk, without popping out of the potential well. It is perhaps more likely that delocalized Ps forms as usual and that some fraction is able to trap at a defect site before breaking apart. In this case, the Ps yield depends on the type and concentration of defects, the Ps diffusion length and on its trapping and oxidation rates. Measurements of the $o$-Ps lifetime as a function of temperature and the width of the p-Ps momentum distribution can be used to estimate the size of the defects in which the ensemble of Ps is trapped. Like the bubble state, the larger the size of the defect in which Ps is trapped the longer the pick-off lifetime and the smaller the zero-point momentum. Equations (3.43) and (3.46) are often used to estimate the defect size.

There exists some experimental evidence to suggest that Ps is formed in the rare-gas solids and that it annihilates from a localized state inside a defect. Tuomissari et al. (1991) implanted positrons into solid $\mathrm{Ar}, \mathrm{Kr}$ and $\mathrm{Xe}$ and then measured the energy distribution for the Ps that diffused back to the surface and was then emitted. Their results clearly show that $\mathrm{Ps}$ is in a delocalized state for the first few picoseconds that it spends in the lattice. Angular correlation experiments measure a $p$-Ps self-annihilation momentum density which is too broad for a delocalized state and indicate a sharply localized state. In fact, angular correlation measurements in the heavier rare-gas solids previous to this work did not resolve the broad $p$-Ps component and instead attributed it to delocalized positron annihilation (Rose and DeBenedetti 1965; Varlashkin 1971). Lifetime experiments in solid neon, argon and xenon (Haberl 1979a,1979b; Tuomisaari et al. 1988) have observed longlived components attributable to $o$-Ps annihilation (see Table 4.3). The o-Ps intensities in solid neon and argon are about $7-8 \%$. These results are consistent with the idea that Ps is formed in a delocalized state and then either traps to a defect within $\sim 5-10$ ps or simply breaks up into a free positron and electron. Schrader et al. (1982) performed lifetime experiments in highly defected matrices of solid argon as a function of isochronal annealing temperature. Their results showed an o-Ps component whose intensity and lifetime decreased as the samples were annealed which is clear evidence that Ps was annihilating from inside pre-formed voids. Extrapolations of these results to well annealed samples are 
consistent with Haberl's $(1979 a, 1979 b)$ data. In the unannealed samples, which contained voids whose size corresponded to a twelve-atom vacancy, the $o$-Ps yield was $\sim 45 \%$ which is significantly more than the Ore gap model alone would predict. The behaviour of the positron lifetime component lead to the suggestion that positrons were also trapped at the voids reacted with spur electrons to either form Ps or annihilate. Such a process could certainly account for the large Ps fraction.

\subsubsection{Positronium Bound States}

The formation of bound states between Ps and atoms (or molecules) in the medium has a significant effect on the Ps annihilation characteristics. Typically, one would expect a bound state to reduce the lifetime and intensity of the long-lived $o$-Ps component in lifetime experiments. The bound state lifetime and momentum density distribution would likely be similar to that for a free positron. Karl et al. (1984) have calculated the possibility of Ps-atom bound states and were unable to find any for the rare-gas atoms. However, their simple model does not provide proof that such states do not exist. Lifetime experiments in moderately dense $\mathrm{Kr}$ and $\mathrm{Xe}$ gas have observed Ps yields that are anomalously below the predictions of the Ore gap model ${ }^{\dagger}$. The lifetime spectra also contain fast annihilation components that have not been observed in $\mathrm{He}$ and $\mathrm{Ne}$, and have only recently been seen in Ar with an electric field applied (Charlton et al. 1992). Heyland et al. (1982) suggested that these results could be explained by the formation of PsKr and PsXe bound states. The difficulty with this is that their interaction is of the van der Waals type which requires no activation energy and so no normal Ps should have been observed. To overcome this, Wright et al. (1985) have suggested the existence of PsKr and PsXe resonant states. In their model. Ps is formed in accordance with the Ore gap predictions. As the Ps thermalizes there are certain energies at which it can temporarily attach to $\mathrm{Kr}$ or $\mathrm{Xe}$ to form a resonant state. Ps having an initial kinetic energy lower than these resonant energies ( $1.7 \mathrm{eV}$ in $\mathrm{Kr}$ and $0.4 \mathrm{eV}$ in $\mathrm{Xe}$ ) annihilates as normal Ps. However, Ps with a higher kinetic energy will annihilate from a resonant state as it passes through the resonant energies during the slowing-down process. The annihilation from resonant states would cause the fast lifetime components and reduce the observed Ps fraction. Angular correlation experiments in the

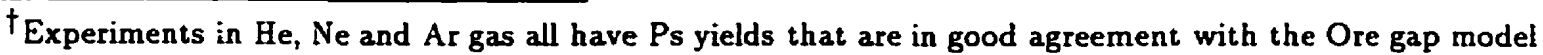
predictions. In $\mathrm{Kr}$ and $\mathrm{Xe}$, the Ore gap model predicts Ps yields of 0.19-0.49 and 0.25-0.59, respectively. The observed Ps fractions in $\mathrm{Kr}$ and $\mathrm{Xe}$ are $0.16-0.28$ and $0.05-0.07$, respectively, depending on the density (Griffith and Heyland 1978) and 0.11 and 0.03 at low densities (Heyland et al. 1982; Wright et al. 1985).
} 
rare gases are also consistent with this model (Kakimoto and Hyodo 1988) . $^{\frac{\hbar}{7} \text {. At higher }}$ xenon gas densities (i.e. $>0.6 \times 10^{22} \mathrm{~cm}^{-3}$ ) Tuomisaari et al. (1988) measured Ps fractions that fell within the Ore gap predictions. It is therefore uncertain if these resonant states also exist in the condensed state.

Another possible bound state that is important to consider is $\mathrm{PsO}_{2}$. Heinberg and Page (1957) showed that a large fraction of the positrons annihilate from a $p$-Ps state in dense $\mathrm{O}_{2}$ gas and suggested that the lack of a long-lived o-Ps component in lifetime experiments was due to o-Ps being converted to $p$-Ps during collisions with $\mathrm{O}_{2}$ molecules (see §3.5.3). In liquid $\mathrm{O}_{2}$, both angular correlation and lifetime experiments fail to see a Ps component. The implication is that either Ps is simply not forming, that it is oxidizing back to a free electron and positron, or that Ps is forming a bound state. Paul (1958) noticed that small amounts of $\mathrm{O}_{2}$ impurity deliberately added to liquid argon had a much greater effect on the o-Ps lifetime and yield than when added to gaseous argon (Deutsch 1953). To explain these results Paul (1959) suggested a Bloch-Bradbury mechanism similar to (3.47)

$$
\mathrm{Ps}+\mathrm{O}_{2}+\mathrm{M} \rightarrow \mathrm{PsO}_{2}+\mathrm{M}
$$

in which excited $\mathrm{PsO}_{2}$ could be formed but would dissociate within a few picoseconds unless it underwent a collisional de-excitation to the ground state. It is likely that in gases most $\mathrm{PsO}_{2}$ would simply autoionize, whereas in liquids almost every collision would be like (3.47). Annihilation from a $\mathrm{PsO}_{2}$ state would give a momentum distribution and lifetime similar to that for a free positron in liquid $\mathrm{O}_{2}$. Schrader and Wang (1976) have calculated Ps affinities to $\mathrm{H}_{2}, \mathrm{~N}_{2}$ and $\mathrm{O}_{2}$ and their results suggest that Ps does not form a bound state with an isolated molecule. Chuang and Tao (1974) also provide evidence that $\mathrm{PsO}_{2}$ does not form in gases, as expected. However, it remains unclear whether or not $\mathrm{PsO}_{2}$ is stable in a liquid.

Finally, we consider the possibility of annihilation from a $\mathrm{Ps}^{-}$state. The stability of a bound system of two electrons and one positron was first predicted theoretically by Wheeler (1946). It was observed experimentally for the first time in a beautiful slow positron beam experiment by Mills (1981). The ground state energy for an isolated ion has been calculated to be $-7.1296 \mathrm{eV}$ (Frolov and Smith 1994) which corresponds to a binding energy

\footnotetext{
FTuomisaari et al. (1988) have offered an alternative interpretation of these results which is also consistent with the available data. They suggested that the fast components were due to o-Ps annihilating at an enhanced rate at energies higher than thermal due to a larger speed and a larger quenching cross section. The quenching cross section could increase with energy since the Ps can penetrate further into the atomic electron clouds which increases the pick-off annihilation rate. However, it is not clear that this would lead to fast components of the type observed.
} 
of $0.3267 \mathrm{eV}$ against breakup into Ps and a free electron. The $2 \gamma$ self-annihilation rate of $\mathrm{Ps}^{-}$ is predicted to be $2.08613 \mathrm{~ns}^{-1}(\tau=0.4794 \mathrm{~ns})$, which is very close to the spin-averaged Ps decay rate, and is in good agreement with the annihilation rate measured by Mills (1983a) of $2.09 \pm 0.09 \mathrm{~ns}^{-1}$.

The possibility of $\mathrm{Ps}^{-}$formation and its interactions in condensed matter has been mentioned several times in the literature (Mogensen et al. 1982). Like Ps, $\mathrm{Ps}^{-}$could form in either an Ore gap or spur type process. However, the possible Ore gap processes

$$
\begin{aligned}
& e^{+}+\mathrm{M} \rightarrow \mathrm{Ps}^{-}+\mathrm{M}^{2+} \\
& \mathrm{Ps}+\mathrm{M} \rightarrow \mathrm{Ps}^{-}+\mathrm{M}^{+}
\end{aligned}
$$

are unlikely since the first reaction requires a double ionization, while the second requires Ps to have a kinetic energy that is typically greater than its binding energy. In either case, the small $\mathrm{Ps}^{-}$binding energy would make for a very narrow Ore gap. If $\mathrm{Ps}^{-}$does form, it is more likely that Ps picks up a spur electron. $\mathrm{Ps}^{-}$formed from $\mathrm{Ps}$ in a delocalized state would have to thermalize and then trap at a defect site in solids or self-trap into a bubble in liquids. The small $\mathrm{Ps}^{-}$binding energy, which is even smaller when it is squeezed in a condensed medium, makes it unlikely that thermalized $\mathrm{Ps}^{-}$could exist in a delocalized state. However, in a large free volume the binding energy would more closely approach the vacuum value. In media where excess electrons do not become localized, it is possible for a spur electron to attach to a Ps already in a localized state. Except for very weakly bound states, it is likely that the $\mathrm{Ps}^{-}$binding energy could be dissipated without leaving the localized state. Based on energy considerations alone, localized $\mathrm{Ps}^{-}$states cannot be dismissed as they are certain to minimize the free energy of the system. For example, the affinity that the heavier rare-gas liquids have for an excess electron (see Table A.3) is more than compensated for by reductions in zero-point energy, the polarization interaction and the bubble surface energy when an excess electron enters a Ps bubble. However, it is unclear if $\mathrm{Ps}^{-}$states can be formed on time scales and in sufficient quantities to be observed experimentally. Lifetime experiments have never distinguished a component with a lifetime of $0.48 \mathrm{~ns}$ - though it is likely that such a component would be lost under the positron component. In liquids, localized $\mathrm{Ps}^{-}$would exist in smaller bubbles than $\mathrm{Ps}$ and would therefore give rise to a wider momentum distribution (assuming that not too much of the momentum was left behind with the remaining electron). In solids, $\mathrm{Ps}^{-}$localized in defects would have a narrower momentum distribution than Ps due to its lower zero-point 
momentum. To date, no angular correlation measurements have attributed a component to annihilations from a $\mathrm{Ps}^{-}$state.

\subsection{The Inhibition and Quenching of Positronium}

There exists a wide range of physical processes and chemical reactions, beyond those already discussed, that can affect both the Ps formation fraction and its annihilation characteristics. These effects are often categorized as either inhibition or quenching mechanisms. Inhibition refers to those processes that reduce the Ps yield while anti-inhibiting processes increase its yield. Quenching mechanisms are those that tend to shorten the o-Ps lifetime. Several of these processes (pick-off annihilation and Ps bound states) have already been discussed. This section will attempt to describe other inhibition and quenching mechanisms relevant to the work in this thesis. Magnetic-field quenching is an important experimental technique for studying Ps properties. In this thesis, static magnetic fields were applied to several condensed rare gases and liquid $\mathrm{N}_{2}$ for the first time. Electric fields, which can affect both Ore gap and spur Ps formation, were used to study Ps in these media. One chemical quenching mechanism of importance is the spin-exchange conversion process, in which $o$-Ps is converted to $p$-Ps and vice-versa. The main concerns are the possibility of spin-exchange collisions with spur electrons and with $\mathrm{O}_{2}$ impurity.

\subsubsection{Magnetic Field Effects}

The use of a magnetic field has long been recognized as an important experimental technique for studying Ps. Application of a static magnetic field perturbs the $o$-Ps $\left(m_{s}=0\right)$ and the $p$-Ps states causing them to mix together, thereby modifying their annihilation characteristics. One effect is to increase the number of $2 \gamma$ Ps self-annihilations. The magnetic field also acts as a physical quenching process - i.e. it reduces the apparent o-Ps $\left(m_{s}=0\right)$ lifetime. The observation of these effects not only gives experimental proof of Ps formation but also allows for the calculation of many of its properties. The effects of a magnetic field on $P s$ in a vacuum or dilute medium are well known (Halpern 1954). What is described below is a straightforward extension to this theory that is useful in describing magnetic 
quenching experiments in many condensed media ${ }^{\dagger}$. The most comprehensive treatment of magnetic-field quenching has been given by Mills (1975).

The interaction of Ps with a constant magnetic field intensity $B_{z}$ modifies the Ps Hamiltonian only by the addition of a spin-dependent term

$$
\dot{\mathbf{H}}^{\prime}=\mu\left(\sigma_{e z}-\sigma_{p z}\right) B_{z}
$$

where $\mu$ is the positron magnetic moment (see Appendix B) and $\sigma_{e z}$ and $\sigma_{p z}$ are the usual Dirac spin matrices, with eigenvalues \pm 1 , for the electron and positron, respectively, along the quantization axis set by $B_{z}$. The operator $\dot{\mathbf{H}}^{\prime}$ acts only on the spin functions $\left|s, m_{s}\right\rangle$ given by (3.4). Applying $\hat{\mathbf{H}}^{\prime}$ to $|1, \pm 1\rangle$ gives zero - i.e. these states remain unperturbed by the field ${ }^{\dagger}$. However, for the $m_{s}=0$ states $\dot{\mathbf{H}}^{\prime}|0,0\rangle=2 \mu B_{z}|1,0\rangle$ and $\dot{\mathrm{H}}^{\prime}|1,0\rangle=2 \mu B_{z}|0,0\rangle$ which means that $|0,0\rangle$ and $|1,0\rangle$ become mixed and are no longer good eigenstates. Upon rediagonalizing the Hamiltonian the new eigenstates, labelled $|-\rangle$ and $|+\rangle$, have definite energies and decay rates in an external magnetic field and may be written as linear combinations of $|0,0\rangle$ and $\{1,0\rangle$

$$
\begin{aligned}
& |-\rangle=\frac{1}{\sqrt{1+y^{2}}}(|0,0\rangle-y|1,0\rangle) \\
& 1+\rangle=\frac{1}{\sqrt{1+y^{2}}}(|1,0\rangle+y|0,0\rangle)
\end{aligned}
$$

where

$$
y=\frac{x}{\sqrt{1+x^{2}}+1}, \quad x \equiv \frac{4 \mu B_{z}}{\Delta E_{\mathrm{Ps}, 1}}
$$

where the energy splitting is approximated by (3.16). The states $|-\rangle$ and $|+\rangle$ converge to $|0,0\rangle$ and $|1,0\rangle$, respectively, as $B_{z} \rightarrow 0$ and are therefore often called the psuedosinglet and psuedotriplet states. The energies of these pseudostates can be written as

$$
E_{ \pm}=\frac{E_{\mathrm{p}-\mathrm{Ps}, 1}+E_{o-\mathrm{Ps}_{\mathrm{s}, 1}}}{2} \pm \frac{\Delta E_{P_{s, 1}}}{2} \sqrt{1+x^{2}}
$$

while the energies for the $|1, \pm 1\rangle$ states remains unchanged. Equation (3.54) shows that there are no linear terms in the Ps energy shift in a magnetic field - i.e. the Zeeman effect is quadratic.

\footnotetext{
†Several experiments in polymers (Bisi et al. 1985), naphthlene and octadecane (Consolati and Quasso 1990) have seen anomalously large quenching effects that cannot be explained using the theory described in this section. Brusa et al. $(1991,1992)$ have extended the theory and shown that Ps weakly interacting with a second unpaired electron is a possible explanation of these results.

$t_{\text {Feinberg et } a l .(1990)}$ show that the $|1, \pm 1\rangle$ states actually do have a very small quadratic Zeeman effect (i.e. about $2 \mathrm{kHz}$ in a 0.1 Tesla field).
} 
The application of a static magnetic field has a significant effect on the Ps annihilation characteristics. The annihilation rates for the $|-\rangle$ and $|+\rangle$ states become

$$
\lambda_{1}=\frac{\lambda_{1}^{o}+y^{2} \lambda_{3}^{o}}{1+y^{2}}, \quad \lambda_{3}=\frac{\lambda_{3}^{o}+y^{2} \lambda_{1}^{o}}{1+y^{2}} .
$$

The annihilation rate for the $|1, \pm 1\rangle$ states remains $\lambda_{3}^{\circ}$. When the magnetic field is large (i.e. $y \rightarrow 1)$ then $\lambda_{1}=\lambda_{3}=\left(\lambda_{1}^{o}+\lambda_{3}^{o}\right) / 2 \approx \lambda_{1}^{o} / 2$ which implies that the psuedotriplet annihilation rate is strongly quenched in a magnetic field.

The mixing of states in a field also affects the fraction of Ps that undergo $2 \gamma$ self annihilation and pick-off annihilation. In the absence of any other quenching mechanisms apart from the magnetic field, the fraction of $2 \gamma$ self annihilations is

$$
F_{2 \gamma, \mathrm{sa}}\left(B_{z}\right)=\frac{\kappa \lambda_{1}^{\mathrm{vac}}}{4\left(1+y^{2}\right)}\left(\frac{1+\varepsilon P}{\lambda_{1}}+y^{2} \frac{1-\varepsilon P}{\lambda_{3}}\right)
$$

where $\varepsilon=2 y /\left(1+y^{2}\right)$ and $P$ is the positron polarization along $H_{2}$ when the Ps is formed. $P=1$ when the polarization is parallel to $H_{z}$ and is -1 when they are antiparallel. The fraction of $2 \gamma$ pick-off annihilations is

$$
F_{2 \gamma, \mathrm{po}}\left(B_{z}\right)=\frac{\lambda_{\mathrm{po}}}{4}\left(\frac{1+\varepsilon P}{\lambda_{1}}+\frac{1-\varepsilon P}{\lambda_{3}}+\frac{2}{\lambda_{3}^{o}}\right)
$$

As briefly mentioned in $\$ 2.2$ the incident positron flux from an external source has a net spin polarization along its direction of motion some fraction of which is conserved during the slowing down process. In diamagnetic insulating materials, such as condensed rare gases, this favours singlet formation when the field is parallel to the direction of motion and triplet formation when it is opposite (Page and Heinberg 1957). The present set of experiments used two positron sources, one above and the other below the sample, so that assuming equal source strength the net spin polarization is zero.

Mills (1975) has also derived an expression for the fraction of $2 \gamma$ self annihilations, which also includes an effective spin-exchange rate $\gamma_{0}$ to model the effects of a wide class of quenching mechanisms with time-independent reaction rates (see below). In this case

$$
F_{2 \gamma, \text { sa }}(x)=\frac{a_{0}+a_{1} x+a_{2} x^{2}}{a_{3}+a_{4} x^{2}}
$$


where

$$
\begin{aligned}
& a_{0}=\kappa \lambda_{1}^{\operatorname{vac}}\left(\lambda_{3}^{o}+\gamma_{o}\right)^{2}\left(\lambda_{3}^{o}+\gamma_{o} / 2\right) \\
& a_{1}=\kappa \lambda_{1}^{\operatorname{vac}}\left(\lambda_{3}^{o}+\gamma_{o}\right)^{2} \lambda_{3}^{o} P \\
& a_{2}=\kappa \lambda_{1}^{\operatorname{vac}}\left(\lambda_{3}^{o}+\gamma_{o}\right)\left[\lambda_{3}^{o}\left(\lambda_{1}^{o}+\lambda_{3}^{o}\right)+\gamma_{o}\left(\lambda_{1}^{o}+3 \lambda_{3}^{o}\right) / 2\right] / 2 \\
& a_{3}=\left(\lambda_{3}^{o}+\gamma_{o} / 2\right)\left(\lambda_{3}^{o}+\gamma_{o}\right)\left[4 \lambda_{1}^{o} \lambda_{3}^{o}+\gamma_{o}\left(\lambda_{1}^{o}+3 \lambda_{3}^{o}\right)\right] \\
& a_{4}=\left[\lambda_{3}^{o}\left(\lambda_{1}^{o}+\lambda_{3}^{o}\right)+\gamma_{o}\left(\lambda_{1}^{o}+3 \lambda_{3}^{o}\right) / 2\right]^{2}
\end{aligned}
$$

and $x=B_{z} / B_{0}$ is defined in (3.53), where $B_{0}=\kappa(36289.0 \pm 0.8) \mathrm{G}$ is half the value used by Mills (1975). If the possibility of spin-exchange is neglected then

$$
F_{2 \gamma, \text { sa }}(x)=\frac{\kappa \lambda_{1}^{\mathrm{vac}}}{4 \lambda_{1}^{o}} \frac{1+x P+x^{2}\left(\lambda_{1}^{o}+\lambda_{3}^{o}\right) /\left(2 \lambda_{3}^{o}\right)}{1+x^{2}\left(\lambda_{1}^{o}+\lambda_{3}^{o}\right)^{2} /\left(4 \lambda_{1}^{o} \lambda_{3}^{o}\right)}
$$

which is equivalent to (3.56) to first order in $\lambda_{3}^{o} / \lambda_{i}^{o}$.

\subsubsection{Electric Field Effects}

Another experimental technique used to study Ps formation and its reactions was to apply an external static electric field to the sample. External electric fields are expected to have no effect on the Ps wave function as they are typically negligible compared to the internal Ps electric field and the strong internal electric fields that exist in condensed matter. Neither external fields nor the internal electric fields of the medium couple to the particle spins in Ps so the descriptions in terms of singlet and triplet states remains valid. The internal fields do affect the spatial part of the Ps wave function by pulling the electron and positron in opposite directions which reduces the electron-positron overlap. This effect has been accounted for phenomenologically by using the internal contact density $\kappa$. Typical external electric fields should have little effect on $\kappa$.

The main effect of an external electric field is on Ps formation. Extensive electric field studies have been done on low-density rare and diatomic gases (Marder et al. 1956; Teutsch and Hughes 1956; Obenshain and Page 1962; Charlton et al. 1992). In all of these studies, the Ps yield rises slowly at low fields before climbing steeply to a maximum saturation value. In the presence of an electric field, the positron may gain or lose energy between elastic collisions. It experiences an acceleration $a=e \mathbf{E} / m_{p}^{*}$ and so the change in energy between collisions is $\frac{1}{2} m_{p}^{*}(v+a t)^{2}-\frac{1}{2} m_{p}^{*} v^{2}=e \mathbf{E}-1+(e E l)^{2} /\left(2 m_{p}^{*} v^{2}\right)$ where $t$ is the time between collisions, $v$ is the positron's velocity at the beginning of the path and $l$ is the 
length of the free path. The term $(e \mathbf{E} \cdot \mathbf{l})$ can be either an energy gain or a loss depending on the directions of $\mathbf{E}$ and $\mathbf{v}$. Over many free paths it averages to zero. However, the positrons will also gain a small amount of energy, on average, due to the $(e E l)^{2} /\left(2 m_{p}^{*} v^{2}\right)$ term. Brandt and Feibus $(1968,1969 \mathrm{~b})$ have used these ideas to show the effect of an electric field on Ore gap Ps formation. They showed that if the energy loss and Ps formation rates were comparable then at low fields there could be a net diffusion of positions out of the Ore gap causing a linear decrease in the Ps yield with field. However, in the rare and diatomic gases, the Ore gap Ps formation rate is so large compared to the energy loss rate (i.e. $\gamma_{r} \ll \gamma_{P_{s}}$ ) that the decrease is not observed. The increase in the average positron energy means that positrons just below the Ore gap can be accelerated back into the gap to have another chance to form Ps. This effect dominates at higher fields and describes the increase in Ps yields in many atomic and molecular gases quite nicely.

The effects of an electric field on the Ps yield in condensed matter is more complicated. Bisi et al. (1983) have performed electric field experiments in various polymers and found that with increasing field the Ps yield at first declined, passed through a minimum which could be very flat, and then at very high fields rose rapidly and approached a saturation value that exceeded the original $E=0$ Ps yield. The initial decline in Ps yield was explained in terms of the spur formation model - the electric field separated some of the positrons and spur electrons which otherwise could have contributed to Ps formation. The flat portion of the Ps yield curves lead Bisi et al. (1983) to conclude that any net diffusion out of the Ore gap was insignificant. The sharp increase in Ps yield at high fields was again well described by the acceleration of positrons back into the Ore gap. Pepe et al. (1993) have performed lifetime experiments in liquid $\mathrm{N}_{2}$ with applied electric fields. They found that the $o$-Ps lifetime remains constant at about $12.5 \mathrm{~ns}$ and that the $o$-Ps intensity drops dramatically from $15.2 \%$ in zero field to $5.3 \%$ at $12.8 \mathrm{kV} / \mathrm{cm}$ and then levels off to about $4.7 \%$ at $32 \mathrm{kV} / \mathrm{cm}$. The shape of their curve is similar to the low field results of Bisi et al. (1983) and was again explained in terms of the spur model. For the relatively small electric field strengths used in these experiments, the Ps yield should not be affected by positrons diffusing out of or accelerating back into the Ore gap. Any changes in the Ps yield are therefore expected to be due to spur processes, which are expected to be more complicated than implied by Bisi et al. (1983) or Pepe et al. (1993). In liquid argon, for example, the free ion (and spur electron) yield increases with electric field (Fuochi and Freeman 1971) as does the electron-ion recombination rate (Shinsaka et al. 1988). Changes 
in the spur Ps yield are due to both increases in spur size (i.e. increased electron-positron separation) and changes in the number of spur species.

\subsubsection{Spin-Exchange Conversion}

Ferrell (1958) has shown that the essential condition for Ps spin-exchange conversions (i.e. o-Ps $=p$-Ps) to occur during elastic collisions is for the quenching agent to contain at least one unpaired electron. Paramagnetic molecules and ions $\left(\mathrm{NO}, \mathrm{O}_{2}, \mathrm{Cu}^{2+}\right.$, $\mathrm{Ni}^{2+}$, etc.), whose non-singlet ground state results in unpaired electrons, have long been known to be effective spin-exchange quenching agents. The condition of unpaired electrons is not essential for inelastic collisions - i.e. a molecule with a singlet ground state can cause Ps conversions via a non-singlet excited state. A detailed description of Ps spinexchange reactions can be found in Appendix E. The experimentally observable effects of these reactions is a relative increase in the number of $p$-Ps annihilations (i.e. p-Ps:o-Ps $>1: 3$ since $o$-Ps converts to $p$-Ps which then annihilates before being converted back to $o$-Ps) and a quenching the o-Ps lifetime. The degree of these effects usually depends on the concentration of unpaired electrons. This work was concerned with the possibility of spin-exchange collisions between $\mathrm{Ps}$ and various spur species and on $\mathrm{O}_{2}$ impurity. In one series of experiments, small quantities of $\mathrm{O}_{2}$ were deliberately added to liquid $\mathrm{Ar}$ and $\mathrm{N}_{2}$ samples to study its effects on the p-Ps yield.

\subsubsection{Ps Conversions With Spur Species}

The condensed rare and diatomic gases have a full closed-shell symmetry for their valence electrons which implies that they are usually unable to cause Ps spin-exchange reactions. However, in experiments using energetic positron sources, the creation of a positron spur produces a number of species which may allow spin-exchange conversions. The low Ps mobility and fast thermalization time (see Appendix C) implies that even in weakly scattering media the Ps remains inside the spur ${ }^{\dagger}$. It has been suggested (see Byakov and Petuchov 1983a) that thermalizing Ps could excite an atom or molecule and then, due to its low mobility, it could interact with the unpaired electron to cause a spin-exchange,

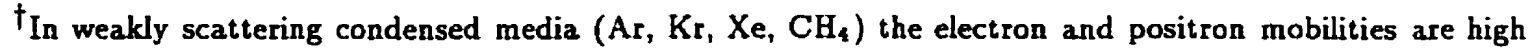
enough that they have a significant probability of escaping the positron spur and the various spur reactions.
} 
or it could interact with an already excited species

$$
o-\operatorname{Ps}(\uparrow \neq)+M^{*}(\downarrow) \leftrightarrow p \cdot \operatorname{Ps}(1 \uparrow)+M^{*}(\uparrow)
$$

Equation (3.61) is an over simplification of the spin-exchange processes - Ferrell (1958) has shown that actual exchange of electrons was not required for Ps conversion (see Appendix E). Typically, the inelastic excitation thresholds are higher than the initial Ps kinetic energy which makes it impossible for Ps to excite an atom or molecule itself ( $\mathrm{O}_{2}$ is an exception). However, it is more likely that the Ps undergoes spin-exchange collisions with excess spur electrons

$$
o-\operatorname{Ps}(\uparrow \uparrow)+\left(\mathrm{e}^{-}\right)^{-}(\downarrow) \leftrightarrow p-\operatorname{Ps}(\downarrow \uparrow)+\left(\mathrm{e}^{-}\right)^{-}(\uparrow)
$$

The reason (3.62) is more likely is that spur electrons can interact with Ps even after it self-traps into a bubble, while the overlap between an excited atom and a Ps bubble is typically negligible. In rare-gas liquids, $p$-Ps is known to annihilate from inside an equilibrium bubble in about 125 ps and is thought to thermalize and then self-trap in about 10 ps (see Appendix C). If spin-exchange collisions only occurred during the first few tens of picoseconds then their effects would not be observable experimentally (i.e. lifetime experiments could not resolve the changes in Ps lifetime and the $p$-Ps:o-Ps ratio would stay close to $1: 3$ ). To account for the experimental observations, the spin-exchange collisions should occur throughout the Ps lifetime at a rate $\gamma_{s e}$ that is comparable to the $p$-Ps selfannihilation rate. If $\lambda_{1} \ll \gamma_{\text {se }}$ then $p$-Ps:o-Ps would remain at $1: 3$, while lifetime experiments would see a single Ps component with the spin-averaged lifetime. Similarly, $\gamma_{\text {se }}<\lambda_{3}$ would have little effect on experimental observations.

The complexity and lack of quantitative information about the spur makes it difficult to model spin-exchange reactions with spur species. A proper description would require a knowledge of their initial concentrations and distribution as well as their spatial and temporal evolution. Reaction rates between spur species and their diffusion constants are also required. The approximations and simplifications required to treat spur reactions are usually significant and often justifiable only in that they give reasonable results. Given these warnings, we proceed to develop a model which should provide some qualitative insight into Ps spin-exchange reactions with spur electrons.

The starting point for the theory is based on the model developed by Byakov and Petuchov (1983b) and Byakov and Grafutin (1986) and has been extended to describe spin- 
exchange reactions in a magnetic field. The following spur reactions are considered to be the most important:

$$
\begin{aligned}
\left\{o-P s+\mathrm{e}^{-}\right. & \left.=p-\mathrm{Ps}+\mathrm{e}^{-}\right\} \\
\{\mathrm{Ps}\} & \rightarrow 2 \gamma \\
\left\{\mathrm{e}_{s}^{-}+\mathrm{M}^{+}\right. & \rightarrow \mathrm{M}\} \\
\left\{\mathrm{e}_{s}^{-}\right. & \left.\rightarrow \mathrm{e}_{\text {bub }}^{-}\right\} \\
\left\{\mathrm{e}_{s}^{-}\right\} & \rightarrow \mathrm{e}_{j}^{-}
\end{aligned}
$$

which describe spin-exchange collisions, annihilation, recombination, electron bubble formation, and the escape of electrons from the spur such that they cannot interact with other spur species.

Using the 'prescribed diffusion' approximation the concentration of the $i^{\text {th }}$ spur species at point $r$ from the centre of the spur and at time $t$ is given by

$$
c_{i}(r, t)=\frac{n_{i}(t) \exp \left(-r^{2} /\left(4 D_{i} t\right)\right)}{\left(4 \pi D_{i} t\right)^{3 / 2}}, \quad t \geq t_{o}
$$

where $n_{i}(t)$ is the number of the $i^{\text {th }}$ species that exist at time $t$ and $D_{i}$ is a diffusion constant. $t_{0}$ is a fictitious time that gives a reasonable initial concentration

$$
c_{i}\left(r, t_{o}\right)=\frac{n_{i 0} \exp \left(-r^{2} / b_{i}^{2}\right)}{\left(\pi b_{i}^{2}\right)^{3 / 2}}
$$

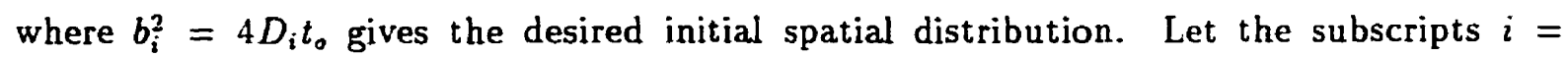
$1,2, \ldots, 8$ label the four Ps spin states in a magnetic field $|-\rangle,|1,-1\rangle,|+\rangle,|1,+1\rangle ;$ (5) spur electrons $e_{s}^{-} ;(6)$ the ions $M^{+} ;(7)$ self-trapped electrons $e_{\text {bub }}^{-}$; and (8) electrons that have escaped the spur $e_{j}^{-}$. The equations describing the concentrations become

$$
\begin{aligned}
& \frac{\partial c_{i}(r, t)}{\partial t}=D_{i} \nabla^{2} c_{i}-\lambda_{i} c_{i}-\sum_{j \neq i} \gamma_{i j} c_{i} c_{5}+\sum_{j \neq i} \gamma_{j i} c_{j} c_{5}, \quad i, j=1,2,3,4 \\
& \frac{\partial c_{5}(r, t)}{\partial t}=D_{5} \nabla^{2} c_{5}-\gamma_{56} c_{5} c_{6}-\gamma_{57} c_{5}-\gamma_{58} c_{8} \\
& \frac{\partial c_{6}(r, t)}{\partial t}=D_{6} \nabla^{2} c_{6}-\gamma_{56} c_{5} c_{6} \\
& \frac{\partial c_{7}(r, t)}{\partial t}=D_{7} \nabla^{2} c_{7}+\gamma_{57} c_{5} \\
& \frac{\partial c_{8}(r, t)}{\partial t}=D_{8} \nabla^{2} c_{8}+\gamma_{58} c_{5}
\end{aligned}
$$

where $\gamma_{i j}(i, j=1,2,3,4)$ are the spin-exchange conversion rates between the four Ps states and an unpaired electron in a magnetic field. The rates are proportional to the matrix 
elements described in Appendix E and may therefore be written as

$$
\begin{aligned}
& \gamma_{i j}=\gamma_{j i}, \quad \gamma_{24}=0 \\
& \gamma_{12}=\gamma_{34}=\alpha^{2} \gamma_{o} \\
& \gamma_{13}=\alpha^{2} \beta^{2} \gamma_{o} \\
& \gamma_{14}=\gamma_{23}=\beta^{2} \gamma_{o}
\end{aligned}
$$

where $\alpha=(1-y) / \sqrt{1+y^{2}}$ and $\beta=(1+y) / \sqrt{1+y^{2}}$ with $y$ defined by (3.53). $\gamma_{0}$ is the spin-exchange conversion rate in zero magnetic field. $\gamma_{56}, \gamma_{57}$ and $\gamma_{58}$ are the reaction rates for electron-ion recombination, electron self-trapping and electron escape from the spur, respectively. The initial number of particles are $n_{10}=\frac{1}{4}(1+\epsilon P), n_{20}=\frac{1}{4}(1-P)$, $n_{30}=\frac{1}{4}(1-\epsilon P), n_{40}=\frac{1}{4}(1+P), n_{50}, n_{60} \gg 1$, and $n_{70}=n_{80}=0$ where $\varepsilon$ is described above and $P$ is the positron polarization.

Substitution of (3.64) into (3.66) and integrating over all space gives

$$
\begin{aligned}
& \frac{d n_{i}(t)}{d t}=-\lambda_{i} n_{i}-\sum_{j \neq i} \frac{\gamma_{i j}}{V_{i 5}} n_{i} n_{6}+\sum_{j \neq i} \frac{\gamma_{j i}}{V_{j 5}} n_{j} n_{5}, \quad i, j=1,2,3,4 \\
& \frac{d n_{5}(t)}{d t}=-\frac{\gamma_{56}}{V_{56}} n_{5} n_{6}-\gamma_{57} n_{5}-\gamma_{58} n_{5} \\
& \frac{d n_{6}(t)}{d t}=-\frac{\gamma_{56}}{V_{56}} n_{5} n_{6} \\
& \frac{d n_{7}(t)}{d t}=\gamma_{57} n_{5}, \quad \frac{d n_{8}(t)}{d t}=\gamma_{58} n_{5}
\end{aligned}
$$

where $V_{i j}=\left(\left(2 \pi b_{i j}^{2}\right)\left(1+t / \tau_{i j}\right)\right)^{3 / 2}$ is the characteristic volume occupied by particles $i$ and $j$ at time $t, b_{i j}^{2}=\left(b_{i}^{2}+b_{j}^{2}\right) / 2, \tau_{i j}=b_{i j}^{2} /\left(4 D_{i j}\right)$ and $D_{i j}=\left(D_{i}+D_{j}\right) / 2$. Obviously $V_{15}=V_{25}=V_{35}=V_{45}$.

In order to make the problem tractable it is necessary to make further simplifications. We assume that

$$
\frac{\gamma_{56}}{V_{56}} n_{6}(t)=\gamma_{56}^{\prime}
$$

which makes (3.68b) linear and homogeneous with respect to $n_{5}(t)$ and so

$$
n_{5}(t)=n_{50} e^{-\gamma_{5} t}
$$

where $\gamma_{5}=\gamma_{56}^{\prime}+\gamma_{57}+\gamma_{58}$. Equation (3.69) gives both the ions and the spur electrons an exponential time dependence, which is reasonable and certainty no worse than other simplifications already included in the theory. For example, the electron-ion recombination 
reactions described above were derived from the usual classical diffusion theory in which the Smoluchowski equation is used to describe the Brownian motion of spur electrons. The application of this equation requires the mean free path of the electron to be small compared to the typical lengths involved in the problem. This requirement is generally fulfilled. However, liquid $\mathrm{Ar}, \mathrm{Kr}$ and $\mathrm{Xe}$ are among the most weakly scattering liquids known (i.e. highest electron mobility) and the spur electron mean free paths are not necessarily small. Tachiya and Schmidt (1989) have shown that for weakly scattering liquids the spur electrons have a significantly greater probability of escaping recombination than is predicted by the diffusion theory. It is impossible, however, to incorporate these results into our simple model.

Given (3.69), the Ps equations become separable from the others so that

$$
\frac{d n_{i}(t)}{d t}=-\lambda_{i} n_{i}-\sum_{j \neq i} \gamma_{i j}(t) n_{i}+\sum_{j \neq i} \gamma_{j i}(t) n_{j} ;, \quad i, j=1,2,3,4
$$

where

$$
\gamma_{i j}(t)=\frac{n_{5 o} \mathrm{e}^{-\gamma^{s} t}}{\left(\left(2 \pi b_{i 5}^{2}\right)\left(1+t / \tau_{i 5}\right)\right)^{3 / 2}} \gamma_{i j}
$$

Given estimates for the parameters in (3.72), the numerical integration of (3.71) is straightforward (Press et al. 1992). The narrow peak in angular correlation experiments due to $2 \gamma$ self-annihilations in an arbitrary magnetic field intensity $B$ is

$$
I_{n}(B)=\frac{\kappa \lambda_{1}^{\text {vac }}}{1+y^{2}} \int_{0}^{\infty}\left(n_{1}(t)+y^{2} n_{3}(t)\right) d t
$$

which now includes the possibility of spin exchange. Positron lifetime experiments in a magnetic field would see two long-lived $o$-Ps components given by

$$
I_{3, \pm 1}(B)=\lambda_{\mathrm{po}} \int_{0}^{\infty}\left(n_{2}(t)+n_{4}(t)\right) d t
$$

due to the $o-\operatorname{Ps}\left(m_{s}= \pm 1\right)$ states and

$$
I_{3,0}(B)=\frac{\lambda_{\mathrm{po}}}{1+y^{2}} \int_{0}^{\infty}\left(n_{3}(t)+y^{2} n_{1}(t)\right) d t
$$

due to pick-off annihilations from the mixed states.

Byakov and Petuchov (1983b) did not assume (3.69) and instead solved (3.68) by assuming that $p$-Ps $\rightarrow o-P s$ was negligible ${ }^{\dagger}$. They showed that the ratio of $p$-Ps to $o$-Ps in

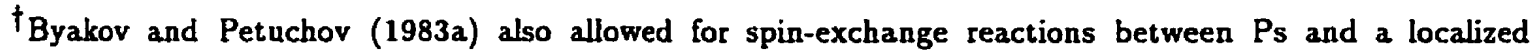
electron, which has been excluded here as unimportant.
} 
zero magnetic field when spin-exchange collisions are possible is

$$
\frac{I_{1}^{\prime}(0)}{I_{3, \pm 1}(0)+I_{3,0}(0)}=\frac{4}{3} \mathrm{e}^{J}-1
$$

where $J$ is given by

$$
J=\frac{\left(\tau_{57}+\tau_{58}\right)\left(1+L_{56} \tau_{25} / \tau_{56}\right) L_{25}}{\tau_{25}+\tau_{57}+\tau_{58}}
$$

where $\tau_{25}$ is the characteristic time for reactions between all $o$-Ps and spur electrons and

$$
L_{i j}=\sqrt{\frac{2}{\pi}} \frac{\gamma_{i j} n_{j o}}{8 \pi D_{i j} b_{i j}}
$$

which typically ranges from 0.1 to 1 and $L_{25}<L_{56}$. When the spur electrons become localized, or leave the spur quickly compared to the spin-exchange process, $\tau_{57}+\tau_{58} \ll \tau_{25}$ so that $J \rightarrow 0$ and so (3.76) approaches one third as expected. However, when the spur electrons remain quasi-free and able to interact with the Ps then $J \approx\left(1+\tau_{25} L_{56} / \tau_{56}\right) L_{25} \approx 1$ which can give a $p$-Ps to $o$-Ps ratio significantly greater than one third.

\subsubsection{The Effects of Oxygen Impurity}

The oxygen molecule has long been recognized as an effective Ps quenching agent (Heinberg and Page 1957). Its ground state is triplet $\mathrm{X}^{3} \Sigma_{g}^{-}$with two unpaired outer electrons having parallel spins. The two lowest excited states are singlet $\mathrm{a}^{1} \Delta_{g}$ and $\mathrm{b}^{1} \Sigma_{g}^{+}$ which are located at 0.977 and $1.627 \mathrm{eV}$ above the ground state, respectively. Experiments in gaseous $\mathrm{O}_{2}$ have shown that the quenching processes there involves two different mechanisms with cross sections that differ by several orders of magnitude (Deutsch and Berko 1965; Kakimoto et al. 1987,1990). The slower process is the usual conversion of o-Ps to p-Ps due to elastic exchange collisions with $\mathrm{O}_{2}$. The elastic conversion cross section is $(1.0 \pm 0.3) \times$ $10^{-19} \mathrm{~cm}^{2}$ (Kakimoto et al. 1990) and is significantly smaller than the atomic dimensions. The second conversion process results from inelastic collisions in which energetic Ps excites $\mathrm{O}_{2}$ into one of its two lowest excited states. In this case, the conservation of spin requires $p$-Ps to convert to o-Ps while o-Ps converts to $p$-Ps one-third of the time (see (E.10)). The inelastic conversion cross section of $\sim 5 \times 10^{-17} \mathrm{~cm}^{2}$ is fast enough that it causes all Ps to thermalize below $1 \mathrm{eV}$ very quickly as well as a quenching of $o$-Ps. Inelastic conversions are not observed for other quenching agents as their inelastic thresholds are usually much higher than the initial Ps kinetic energy. The addition of $\mathrm{O}_{2}$ impurity to a sample is also known to have a significant effect on the Ps formation fraction. Several experiments in high-density 
argon gas observed a smooth transition in the Ps yield from that in pure argon gas (33\%) to that in pure $\mathrm{O}_{2}$ gas (45\%), as the $\mathrm{O}_{2}$ impurity concentration was increased (Mokrushin and Goldanskii 1968; Klobuchar and Karol 1980). Lifetime experiments in liquid Ar and $\mathrm{N}_{2}$, with small quantities of $\mathrm{O}_{2}$ impurity deliberately added, observed a quenching of the o-Ps lifetime and an increase in the o-Ps intensity (Paul 1958; Spektor and Paul 1971a). The $o$-Ps annihilation rate increased linearly with $\mathrm{O}_{2}$ concentration, as expected for any simple quenching mechanism. In liquid argon, the $o$-Ps lifetime drops from $7.0 \mathrm{~ns}$ in pure $\mathrm{Ar}$ to $1.4 \mathrm{~ns}$ at 8.0 torr $\mathrm{O}_{2}$ partial pressure. The o-Ps intensity initially rises from $7.0 \%$ in pure $\mathrm{Ar}$ to $10.0 \%$ with 0.5 torr $\mathrm{O}_{2}$ partial pressure and increases linearly from there to $14.6 \%$ at 8.0 torr $\mathrm{O}_{2}$ partial pressure. In liquid $\mathrm{N}_{2}$, the $o$-Ps lifetime drops from about $12.5 \mathrm{~ns}$ in pure $\mathrm{N}_{2}$ to about 3 ns with 5.0 torr of $\mathrm{O}_{2}$ partial pressure. The increase in the o-Ps intensity is much smaller, rising non-linearly from $13.9 \%$ in pure $\mathrm{N}_{2}$ to $14.3 \%$ at 5.0 torr of $\mathrm{O}_{2}$, and appears to be approaching a limit at higher $\mathrm{O}_{2}$ concentrations.

The addition of $\mathrm{O}_{2}$ impurity can affect both the Ore gap and the spur formation processes, especially in the condensed rare gases. Molecular impurities typically have inelastic scattering thresholds below those of the condensed rare gases. This has two main effects on Ore gap Ps formation. Inelastic scattering with impurities could allow positrons hung up in the Ore gap to escape below the Ps formation threshold more easily, thereby reducing the Ore gap $P$ s yield. However, for this to be a significant effect would require either large impurity concentrations or an inelastic impurity scattering cross section that was large compared to the Ps formation cross section. The Ore gap Ps cross section in the rare gases is comparable to the geometric cross section making it unlikely that impurity scattering out of the Ore gap is significant for small impurity concentrations. A more significant effect is that an impurity produces a second Ore gap in the sample between $E_{I, l}=E_{I, \text { ion }}-E_{\mathrm{Ps}, \mathrm{b}}<E_{p}<E_{I, \mathrm{u}}=\min \left(E_{l}, E_{I, \text { ion }}\right)$, where $E_{l}$ is the original Ps formation threshold, $E_{I, \text { ion }}$ is the impurity ionization potential in the condensed medium, and $E_{I, l}$ and $E_{I, u}$ are the lower and upper bounds of the impurity Ore gap (Brandt and Feibus 1969b). If $E_{I, l}<E_{l}$ then it is possible for positrons that have fallen below the original Ps formation threshold to still form Ore gap Ps in a collision with an impurity. The impurity-induced Ps yield obviously depends on the positioning and width of the impurity Ore gap, the impurity concentration, the positron energy loss rate and on the impurity Ps formation cross section. For $\mathrm{O}_{2}$ impurity in the condensed rare gases, the positron energy loss rate below $E_{l}$ is so low that impurity-induced Ps formation could be important. The thermalization rates 
in molecular media, such as $\mathrm{N}_{2}$, are fast enough that small concentrations of $\mathrm{O}_{2}$ impurity should not have a significant effect on the Ore gap Ps yield.

Molecular impurities in the condensed rare gases also increase the positron and spur electron thermalization rates. Results presented in Appendix C suggest that both positrons and excess electrons in the heavier rare gases thermalize in times comparable to the positron lifetime. If this is correct, then the spur Ps yield is expected to be small. At low concentrations, molecular impurities should reduce the thermalization times and the ranges (i.e. the spur size) for both positrons and spur electrons, thereby increasing spur Ps formation. However, as the impurity concentration increases the range of the spur electrons becomes smaller making it more difficult for them to escape the Coulomb field of their parent ions. At high enough impurity concentrations, the positron spur may simply collapse too quickly - i.e. the spur electrons simply recombine with their ions and are therefore unavailable for spur $\mathrm{Ps}$ formation. This is especially true for $\mathrm{O}_{2}$ impurity whose low energy excitations make it very effective at thermalizing light particles. Fuochi and Freeman (1972) added $\mathrm{O}_{2}$ to liquid argon and observed a free ion yield ${ }^{\dagger}$ and an average electron-ion separation that decreased with $\mathrm{O}_{2}$ concentration, as shown in Table 3.1. These results also lead to the conclusion that the thermalization effects of the $\mathrm{O}_{2}$ impurity was more important than spur electron attachment to $\mathrm{O}_{2}$ molecules.

In this work, small quantities of $\mathrm{O}_{2}$ impurity were deliberately added to liquid Ar and $\mathrm{N}_{2}$ samples to study its effects on Ps. Assuming that the effects of changing Ps yield can be separated from spin-exchange conversion, the model developed above for spur species can be used with $\mathrm{O}_{2}$. However, unlike the spur species, the $\mathrm{O}_{2}$ concentration remains constant both in time and spatially, which leads to considerable simplification. In this case, (3.71) can be solved with time-independent conversion rates $\left(i . e . \gamma_{i j}(t) \rightarrow \gamma_{i j}\right)$. The resulting equations

\footnotetext{
$t_{T h e}$ free ion yield $G_{\mathrm{f}}^{\circ}$ is usually defined to be the number of pairs of free ions formed per $100 \mathrm{eV}$ of energy absorbed by the system in zero electric field. $G_{t o r}^{o}$ is the total yield of (3.20) per $100 \mathrm{eV}$. The two quantities are related by $G_{\mathrm{f}}^{o}=\phi_{\mathrm{f}}^{\circ} \mathrm{G}_{\mathrm{ios}}^{\circ}$ where $\phi_{\mathrm{f}}^{\circ}$ is the fraction of ions that escape recombination at zero electric field. In the usual diffusion theory it is estimated by

$$
\phi_{\mathrm{f}}^{\circ}=\int_{0}^{\infty} \dot{\phi}_{\mathrm{h}}^{\circ}(r) F(r) d r
$$

where $F(r)$ is the initial spur electron distribution and is usually approximated by a three-dimensional Gaussian and

$$
\phi_{\AA}^{\circ}(r)=\exp \left(-\frac{r_{c}}{r}\right), \quad r_{c}=\frac{\mathrm{e}^{2}}{4 \pi \epsilon k_{B} \bar{T}}
$$

where $r_{c}$ is the Onsager radius corresponding to the distance at which the Coulomb field between the ion and spur electron equals the average thermal energy $k_{B} T$. Tachiya and Schmidt (1989) have shown that this estimate for $\phi_{\mathrm{i}}^{\circ}$ underestimates $G_{\mathfrak{f}}^{\circ}$ in weakly scattering liquids.
} 
Table 3.1: The free ion yields $G_{\mathrm{f}}^{o}$, total ion yields $G_{\text {tot }}^{o}$, and average initial electron-ion separations $b$ taken from Fuochi and Freeman (1972). The results in brackets are more current estimates for pure argon and $\mathrm{O}_{2}$ from Freeman (1987).

\begin{tabular}{lccc}
\hline Liquid & $\mathrm{G}_{\mathfrak{f}}^{\circ}$ & $\mathrm{G}_{\text {tot }}^{o}$ & $b(\AA)$ \\
\hline Ar (pure) & $2.0(2.7)$ & $5.4(4.5)$ & $1300(2470)$ \\
+1.5 mole\% $\mathrm{O}_{2}$ & 0.7 & $\sim 5$ & 570 \\
+13 mole\% $\mathrm{O}_{2}$ & 0.3 & $\sim 5$ & 380 \\
+28 mole\% $\mathrm{O}_{2}$ & 0.2 & $\sim 5$ & 320 \\
$\mathrm{O}_{2}$ (pure) & 0.013 & $\sim 4$ & $158(110)$ \\
\hline
\end{tabular}

are precisely those used by Bisi et al.(1981,1987) to study the quenching effects of various paramagnetic ions on $\mathrm{Ps}$ in a magnetic field. For $\mathrm{O}_{2}$, the magnetic field dependence of the $\gamma_{i j}$ will be different from (3.67) but can be easily derived by following the ideas developed in Appendix E. Unfortunately, the angular correlation experiments used in this work are unable to resolve any differences in momentum between the elastic and inelastic quenching processes and so only an effective spin-exchange rate can be obtained. If the Ps spinconversion rates are assumed to have no magnetic-field dependence then (3.58) can also be used to find $\gamma_{o}$. In zero magnetic feld, our spin-conversion model simplifies enough that it can be solved analytically and becomes equivalent to an often used model originally derived by Dixon and Trainor (1955). 


\section{Chapter 4 \\ The 1D-ACAR Experiments and Results}

There are three common experimental techniques used to study positron annihilation: measurements of the angular correlation of the annihilation radiation (ACAR); positron annihilation lifetime (PAL) spectroscopy and Doppler-broadened annihilation radiation lineshape (DBARL) measurements. The more recent use of slow positron beams is also beginning to provide a wealth of interesting and useful information. All of the experimental results obtained in this thesis were measured using a high resolution long-slit ACAR apparatus. The apparatus is described in some detail in $\$ 4.1$. The more modern $2 \mathrm{D}$-ACAR apparatus is briefly mentioned in \$4.1.3. All of the relevant, previous experimental ACAR data is summarized in $\$ 4.2$. The experimental results obtained in this thesis are discussed in §4.3. This includes a description of the data corrections and some simple preliminary analysis. The results will be analyzed in more detail in the chapters 5 and 6 . Sections 4.4 , 4.5 and 4.6 briefly describe the positron lifetime, Doppler-broadening, and slow-positron beam techniques and summarizes all the known relevant experimental results from each of them.

\subsection{The ACAR Experiments}

The motion of an annihilating electron-positron pair results in an angular deviation of the $\gamma$-rays from $180^{\circ}$, as described in $\$ 2.5 .1$. In angular-correlation studies one or more components of the annihilating pair's $2 \gamma$ momentum density distribution is obtained by measuring these angular deviations. The apparatus consists of a positron emitting radioisotope placed close to a sample and positioned precisely midway between two $\gamma$-ray detectors. Some fraction of positrons from the source penetrate into the sample, thermalize and eventually annihilate there. The resulting $0.511 \mathrm{MeV} \gamma$-rays are then detected in coincidence with the angular deviations being measured using one of a variety of different detector setups. 


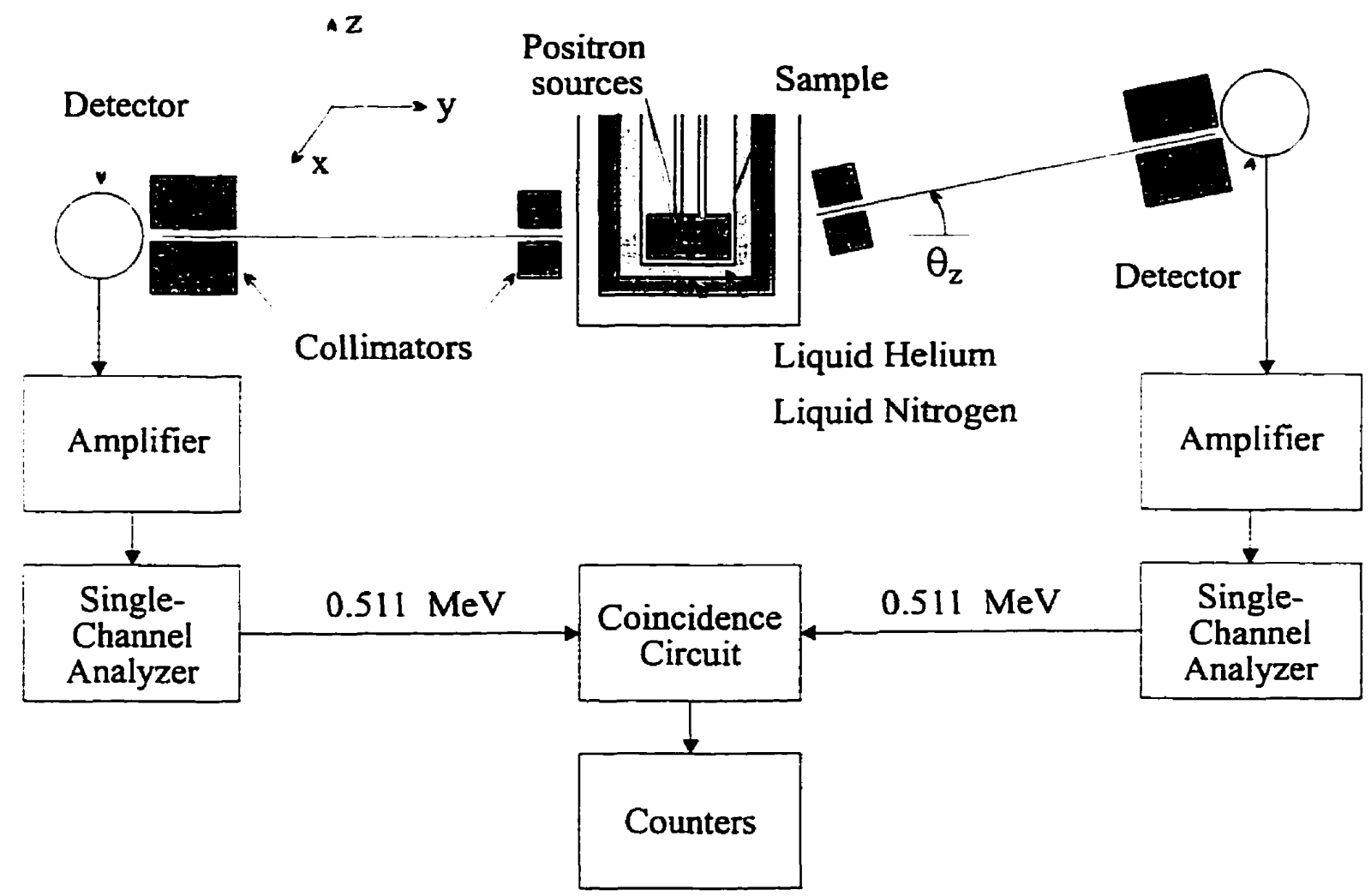

Figure 4.1: A conventional long-slit ACAR apparatus used to measure the angular correlation of the $2 \gamma$-annihilation photons.

\subsubsection{The 1D-ACAR Apparatus}

The most common detector setup, and the one used in this thesis, for achieving a good resolution with adequate counting rates is the long-slit geometry. The apparatus is shown schematically in Figure 4.1. Each $\gamma$-ray detector consists of two cylindrical NaI scintillation crystals $(5 \mathrm{~cm}$ dia. $\times 60 \mathrm{~cm}$ ) each coupled to a photomultiplier (PMT). High resolution in the $z$ direction is achieved by placing the detectors behind long narrow collimating slits and by using a large sample-to-detector distance. The current apparatus used a sample-to-detector distance of 500 inches $(12.70 \mathrm{~m})$ allowing it to achieve the highest resolution of any known ACAR apparatus, and limited only by the positron's thermal motion. Other horizontal collimating slits on either side of the cryostat shielded the detectors from direct view of the positron sources and defined the sample volume seen by the detectors. Each detector subtends a large angle in the $x$ direction ( $\sim 96$ mrads), as compared to the total width of a momentum distributions ( $\sim 30 \mathrm{mrads}$ ), in order to achieve both adequate counting rates and a true one-dimensional projection. Complicated corrections must be 
applied if the detector length cannot be considered as infinite to a good approximation (Mijnarends 1969; Mogensen 1970). One consequence of the large subtending angle in the $x$ direction is that any information concerning the $p_{x}=\theta_{x} m_{o} c$ momentum component is lost. Similarly, the Doppler shift in the $\boldsymbol{p}_{\boldsymbol{y}}$-direction is small compared to the energy resolution of the detectors ( $\sim 50 \mathrm{keV}$ for $\mathrm{NaI}$ ) so that any $p_{y}$ information is also lost. The 1D-ACAR apparatus therefore simply counts the number of coincident $2 \gamma$ annihilations as a function of the $\theta_{z}$ position. The $2 \gamma$ coincidence counting rate (assuming perfect resolution) is given by

$$
N_{\mathrm{ex}}\left(p_{z}\right)=C \int_{-\infty}^{+\infty} \int_{-\infty}^{+\infty} \rho_{\mathrm{ex}}\left(p_{x}, p_{y}, p_{z}\right) d p_{x} d p_{y}
$$

where $C$ is an arbitrary normalization constant, $\rho_{\text {ex }}(\mathbf{p})$ is the annihilating electron-positron pair momentum density and $p_{z}=\theta_{z} m_{0} c . N_{\text {ex }}\left(p_{z}\right)$ is simply the probability that the annihilating pair has a $z$-momentum component between $p_{z}$ and $p_{z}+d p_{z}$ and is known as the 1D-ACAR curve. $N_{\text {ex }}\left(p_{z}\right)$ may also be written in as

$$
N_{\mathrm{ex}}\left(p_{z}\right)=C \int_{0}^{2 \pi} \int_{p_{z}}^{\infty} \rho_{\mathrm{ex}}\left(p, p_{\phi}, p_{z}\right) p d p d \phi
$$

where (4.1) was first transformed to cylindrical coordinates with $p_{x y}^{2}=p_{x}^{2}+p_{y}^{2}$ and $p^{2}=$ $p_{x y}^{2}+p_{z}^{2}$ and then transformed again using $p_{x y} d p_{x y}=p d p$. The actual counting rate is obtained by deconvoluting $N_{\text {ex }}\left(p_{z}\right)$ with the instrumental resolution function (Appendix F).

To measure the angular deviations one detector remained fixed at the height of the sample while the other detector, and its collimating slits, was able to move in the $z$ direction. The movable detector was mounted so that it traveled along two vertical threaded shafts driven by a stepping motor in increments of $0.100 \pm 0.005$ mrads with a maximum range of about \pm 20 mrads. The detector mounting ensured that it always looked radially at the sample as it moved away from the central position and that the sample-to-detector distance remained constant. A second stepping motor kept the collimating slits on the movable detector side of the cryostat aligned with the detector slit. A microcomputer controlled both stepping motors and monitored the movable detector's position using an encoder attached to the top of one of the threaded shafts.

The electronics used in the apparatus are relatively simple. $\gamma$-rays entering the scintillators result in visible light being emitted. The light output of each scintillator, which is proportional to the energy lost as the $\gamma$-ray crossed through it, was collected by the PMTs. The output from a pair of PMTs making up a detector was fed into a common 
charge-sensitive preamplifier which in turn was connected to an amplifier and a timing single channel analyzer (TSCA). The TSCAs' discriminator windows were set to accept pulses in the 100 to $600 \mathrm{keV}$ range, thus selecting out the pulses corresponding to $511 \mathrm{keV}$ annihilation $\gamma$-rays. The output from each TSCA, which occurred at a precise time with respect to the input pulse, was then fed into a fast coincidence circuit. The coincidence circuit simply provided an output logic pulse whenever signals appeared on its inputs within a preset resolving time of $2 \tau=110 \mathrm{~ns}$. The singles and coincidence counting rates were monitored on nuclear counters. In a typical experiment, the collimating slits and sample would be aligned first, with care taken to ensure that the detectors did not see the positron sources directly. The microcomputer would then be programmed to stop at some 20-30 positions between \pm 15 mrads, with a higher density of stops in the regions of interest around $\theta_{z}=0$. An interface module allowed the microcomputer to start, stop and read each of the counters thereby making the data collection process completely automatic. Each experiment was typically the sum of 3-10 similar scans of the movable detector through all the preset stop positions in order to minimize errors due to electronic drift and source decay.

\subsubsection{The Sample and Source Arrangement}

The condensed gas samples and positron sources were contained inside a standard double dewar cryostat placed precisely between the two detectors. Down the central axis of the inner chamber was a cylindrical fiberglass thimble $(5 \mathrm{~cm}$ dia. $\times 115 \mathrm{~cm})$ which isolated the sample chamber from the cryogenic fluid. External magnetic fields could be applied to the samples using a niobium titanium superconducting solenoid hung from a support system inside the inner dewar. The solenoid, which required cooling to liquid helium temperature, was capable of generating flux densities of up to $7.00 \pm 0.01$ Tesla. Beam ports inserted into the magnet and cryostat walls served to minimize the $\gamma$-ray attenuation. A conventional roughing and diffusion pumping system allowed the thimble, sample chamber and gastransfer systems to be pumped down to $10^{-6}$ torr.

To form the condensed gas samples it was necessary to construct a sample chamber and support structure that could be inserted into the cryostat and a gas-transfer system. The cylindrical sample chamber $(3 \mathrm{~cm}$ dia. $\times 2 \mathrm{~cm}$ ) was originally made from stainless steel but was later replaced by one made of copper, which has a significantly higher thermal conductivity, to ensure a more uniform temperature throughout the sample. The chamber 
was supported from the top of the cryostat by a thin-walled stainless steel tube connected to a gas-exchange system outside the cryostat. Using a special insertion tool, a positron source soldered onto a screw could be inserted into a threaded hole centred on the bottom of the chamber. A second positron source was supported in the sample chamber by a thin-walled stainless steel tube running down inside the sample chamber support tube. Electric fields could be generated in the sample between the positron sources by applying a high voltage to the inner tube, which was electrically insulated from the grounded outer tube and sample chamber. Calibrated threaded posts at the top of the cryostat allowed the height of the sample chamber and the source separation to be varied independently to within $\pm 0.1 \mathrm{~mm}$. The source separation was typically $2.5-3.0 \mathrm{~mm}$. The sample chamber was positioned to ensure that the collimating slits always hid the positron sources from a direct view of the detectors. O-ring seals at the top of the cryostat prevented atmospheric leaks into either the thimble or the sample chamber.

The sample temperature was maintained using a commercial temperature controller connected to a silicon diode temperature sensor mounted on the bottom of the sample chamber. The temperature calibration of the diode was accurate to about $\pm 0.1 \mathrm{~K}$ in zero magnetic field and $\pm 0.2 \mathrm{~K}$ in large fields. The controller heated the sample through a nichrome wire wrapped around the sample chamber. Small amounts of helium ( $50 \mathrm{mtorr}$ ) in the thimble acted as a conduction gas to cool the sample. Once a steady state was reached the controller was able to maintain the sample temperature to within $\pm 0.1 \mathrm{~K}$ over the course of an experiment.

The purpose of the gas-transfer system was to deliver a known quantity of gas to the sample chamber where it would be condensed for the experiment. The transfer system consisted of a cylinder of sample gas connected in series to a large volume pressure tank, a gas purifier, a small volume pressure tank and then to the sample chamber support tube. High vacuum valves allowed each component of the transfer system to be isolated from the others. All volumes within the system were accurately measured. The gas pressure was measured using a Bourdon gauge accurate to \pm 5 torr. The procedure for transferring gas to the sample chamber consisted of first pumping the entire system down to $\sim 10^{-7}$ torr for several days prior to an experiment. The cryostat insert was then isolated from the gasexchange system and the two positron sources inserted into sample chamber. During this transfer procedure, a constant stream of helium gas was blown out of the sample chamber and support tubes to minimize the impurities that could get in. Once the transfer was 
complete, the sample chamber was again pumped down to $10^{-7}$ torr and left pumping for another hour in an effort to remove any impurities. To ensure that enough condensed sample formed it was necessary to calculate the quantity of room temperature gas required to completely fill the sample chamber with condensed sample at the desired temperature and maintain a vapour pressure above it. Sample gas from the cylinder was then allowed to pressurize the large volume pressure tank with the required quantity of gas, which was then allowed to flow into the sample chamber and condense. It typically took about an hour for enough liquid sample to form and for its temperature and vapour pressure to stabilize. The solid Ne and Ar samples were formed by slowly (i.e. 1-2 hours) cooling from the liquid to within several degrees below the melting point. Under these growing conditions, the samples would be polycrystalline with a grain size of $\sim 0.1 \mathrm{~mm}$ and likely contain a large number of defects (Venables and Smith 1977).

One of the important experimental concerns was the purity of the condensed gas samples. The most likely impurities in both the gas-exchange system and the gases themselves were $\mathrm{V}_{2}, \mathrm{O}_{2}$ and $\mathrm{H}_{2} \mathrm{O} . \mathrm{O}_{2}$ impurity is known to have a significant effect on Ps lifetimes and yields as described above. The effects of $\mathrm{N}_{2}$ impurity are significantly less severe (Swan 1963). For the liquid He samples, impurities were not expected to be a problem since the solubility of all substances in liquid He is very small. Furthermore, for both the He and Ne samples, most impurities are likely to have been frozen out onto the walls of the $1.3 \mathrm{~m}$ long support tubing. To minimize atmospheric leaks, the entire system was carefully checked with a helium leak tester and was capable of maintaining pressures of $10^{-6}$ torr for several weeks. To reduce the impurities an inert gas purifier (Matheson Gas model 6406) was installed into the gas-exchange system. It used a copper reactant imbedded on high surface area alumina as the $\mathrm{O}_{2}$ and water getters. The purifier was capable of removing $\mathrm{O}_{2}$ and $\mathrm{H}_{2} \mathrm{O}$ at levels of up to $50 \mathrm{ppm}$ in inert gases and maintaining less than $1 \mathrm{ppm}$ at the outlet. The helium gas used in the experiments was the Canox high purity grade (minimum purity $99.995 \%$ ) containing $\mathrm{O}_{2}<3 \mathrm{ppm}, \mathrm{H}_{2} \mathrm{O}<5 \mathrm{ppm}$ and less than $50 \mathrm{ppm}$ total impurity. Ultra high purity (minimum purity $99.996 \%$ ) neon gas was used with $\mathrm{O}_{2}<1$ pp$\mathrm{m}, \mathrm{H}_{2} \mathrm{O}<5 \mathrm{ppm}$ and less than $40 \mathrm{ppm}$ total impurity. The high purity argon gas had $\mathrm{O}_{2}<10 \mathrm{ppm}, \mathrm{H}_{2} \mathrm{O}<10 \mathrm{ppm}$, and less than $50 \mathrm{ppm}$ total impurity. Finally, the nitrogen gas that was used was an oxygen-free grade (minimum purity $99.99 \%$ ) with $\mathrm{O}_{2}<3 \mathrm{ppm}$ and total impurities less than $100 \mathrm{ppm}$.

Several experiments involved deliberately mixing a known quantity of $\mathrm{O}_{2}$ impurity 
with the liquid $\mathrm{Ar}$ and $\mathrm{N}_{2}$ samples. For these experiments the gas purifier was bypassed and the liquid samples formed as described above. A known quantity of $\mathrm{O}_{2}$ gas was then added to a small calibrated volume that could be isolated from the rest of the gas-exchange system. The pressure in this volume was measured using a calibrated strain gauge transducer accurate to \pm 2 torr. To ensure complete mixing, the liquid sample was heated back to gas and allowed to mix with the $\mathrm{O}_{2}$ before being recondensed. Unfortunately, the solubility of $\mathrm{O}_{2}$ in the rare-gas liquids and in liquid $\mathrm{N}_{2}$ is not known. Thus, while the mole fraction of $\mathrm{O}_{2}$ in the gaseous mixture is well-known, the relative fractions of $\mathrm{O}_{2}$ impurity dissolved in the liquid and remaining in the vapour are unknown. However for liquid $\operatorname{Ar}$ and $\mathrm{N}_{2}$, the relatively small $\mathrm{O}_{2}$ concentrations make it reasonable to assume that all of the impurity dissolves into the liquid.

The positron sources typically used in ACAR experiments are ${ }^{22} \mathrm{Na},{ }^{58} \mathrm{Co}$, or ${ }^{64} \mathrm{Cu}$ with activities ranging from $\sim 0.01-1 \mathrm{Ci}$. These experiments used ${ }^{64} \mathrm{Cu}$. Each source was cut from $0.025 \mathrm{~mm}$ thick $99.999 \%$ pure ${ }^{63} \mathrm{Cu}$ foil and irradiated for a 24 hour period in the NRU reactor at Chalk River Laboratories. ${ }^{63} \mathrm{Cu}$ has a high thermal neutron capture crosssection resulting in the production of the radioisotope ${ }^{64} \mathrm{Cu}$. About $18 \%$ of this isotope undergoes $\beta^{+}$decay with a 12.70 hour half-life (see Table B.2 and Figure B.1). The initial source activity was about $1 \mathrm{Ci}$, which gave reasonable counting statistics for about two days worth of experiments. This typically allowed 3-5 different experiments to be performed, each having an ACAR curve with 3000-5000 coincidence counts at $\theta_{z}=0$.

The two source arrangement pictured in Figure 4.1 was used for several reasons. A single source would have resulted in the detectors seeing an exponential implantation profile, as described by (2.1). Small changes in the source position, due to thermal expansion or contraction in the support tubes, could have significantly affected on the counting rates during an experiment. However with two sources, the positron intensity in the defined sample volume between the sources was always reasonably constant. The two source arrangement was also useful in the magnetic field experiments. Positrons emitted from a $\beta^{+}$source are spin polarized along their momenta and are only partially depolarized during the slowing down process (Bisi et al. 1962). As can be seen from (3.56) Ps formation in a magnetic field depends on the residual spin polarization of the positron. However by using two sources, the net spin polarization reduces to approximately zero, and the Ps substates form initially in the expected fractions. 


\subsubsection{D-ACAR Experiments}

More recently, two-dimensional angular-correlation machines have been developed to overcome the considerable loss of information that results from the double integration in (4.1) or (4.2). These detection systems are based on either arrays of scintillation counters (Berko 1983) or multi-wire proportional chambers (Manuel 1983) and are able to resolve two of the three momentum components. The coincidence counting rate for these $2 \mathrm{D}$-ACAR systems is given by

$$
N_{\mathrm{ex}}\left(p_{x}, p_{z}\right)=C \int_{-\infty}^{+\infty} \rho_{\mathrm{ex}}\left(p_{x}, p_{y}, p_{z}\right) d p_{y}
$$

where the $p_{y}$ integral again results from the detector's insensitivity to the small Doppler shift of the $\gamma$-rays. As before, the actual $2 \mathrm{D}$-ACAR surface is obtained from a $2 \mathrm{D}$ convolution of $N_{\text {ex }}\left(p_{x}, p_{z}\right)$ with the instrumental resolution function. To date, no 2D-ACAR measurements have been taken in the condensed rare or diatomic gases and so this experimental setup will not be discussed in any further detail.

The effect of the different detector geometries can be seen by applying (4.2) and (4.3) to a spherical momentum distribution

$$
\rho_{2 \gamma}(\mathbf{p})= \begin{cases}\text { const } & p \leq p_{F} \\ 0 & p>p_{F}\end{cases}
$$

where $p_{p}$ is the Fermi momentum. This particularly simple model corresponds to a positron in an electron gas with all Coulomb interactions turned off. For the long-slit geometry the $1 D$-ACAR curve gives an inverted parabola with a cutoff at $p_{z}= \pm p_{F}$. The 2D-ACAR surface predicts an ellipsoid, symmetric about the $p_{x}$ axis, bounded in the $\left(p_{x}, p_{z}\right)$-plane by $p_{x}^{2}+p_{z}^{2}=p_{F}^{2}$.

\subsection{Previous ACAR Results}

A typical 1D-ACAR curve consists of several momentum distributions, one for each 'type' of electron in the sample and each of the form given by (4.1). A free positron in thermal equilibrium with the sample has negligible momentum so the measured momentum is largely due to the annihilating electron. Positron annihilation with valence electrons results in a Gaussian shaped momentum distribution with a FWHM of 5-10 mrads. Annihilation with conduction electrons gives a somewhat narrower, inverted parabolic momentum 


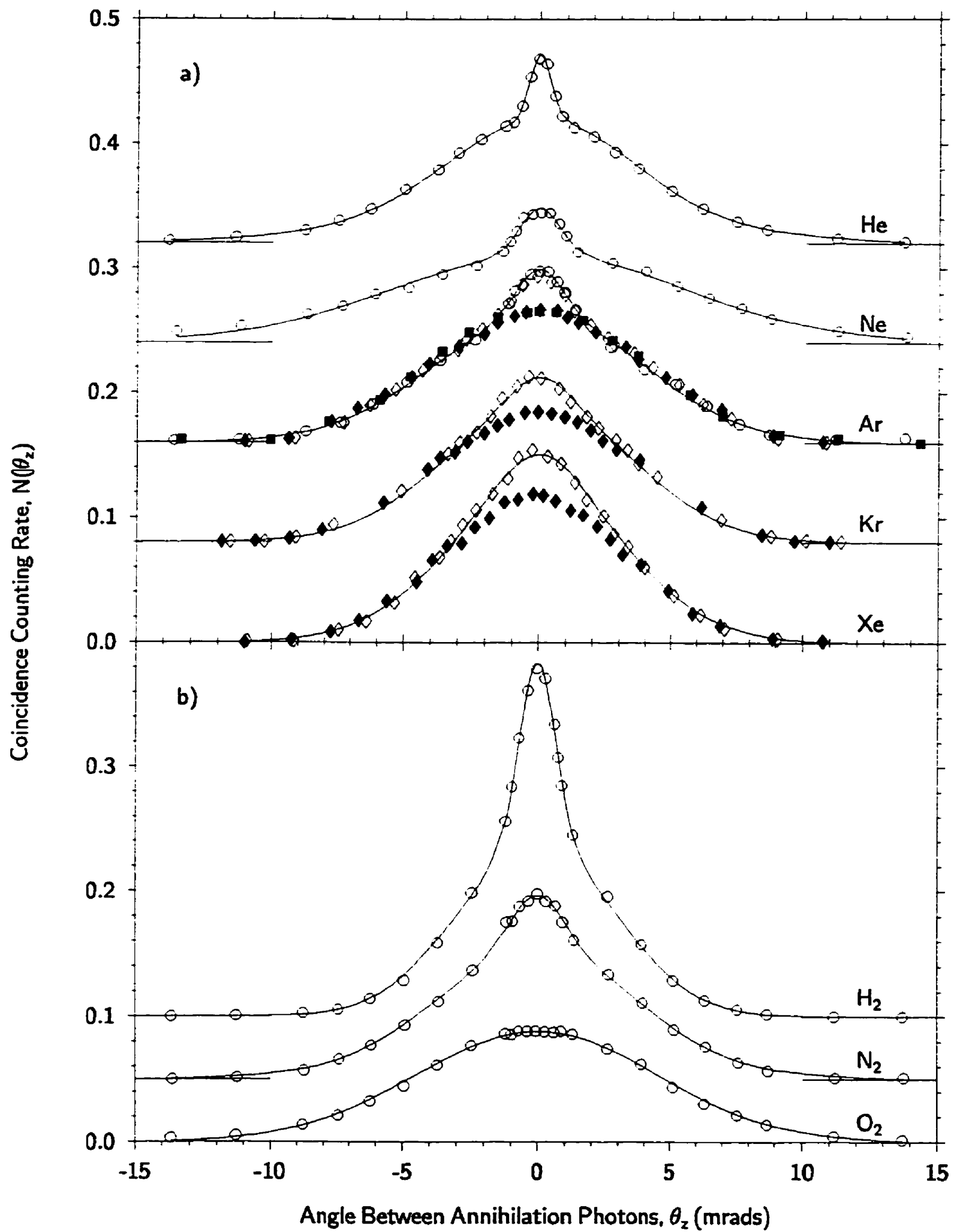

Figure 4.2: 1D-ACAR spectra in: a) the condensed rare gases; and b) liquid $\mathrm{H}_{2}$, $\mathrm{N}_{2}$, and $\mathrm{O}_{2}$. The circles are data taken from Briscoe and Stewart (1967) and Briscoe et al. (1968). Varlaskin's (1971) results in solids and liquids are given by the triangles and diamonds, respectively. The squares from Rose and Debenedetti's (1965) measurement in solid argon. The solid lines are obtained from a two or three Gaussian fit to the data and are intended to simply aid the eye. 
Table 4.1: A summary of the experimental $2 \gamma$ 1D-ACAR results for the condensed media of interest to this thesis. These results have typically been analyzed assuming only two momentum components. The narrow component, resulting from $p$-Ps self-annihilations from a localized bubble state in liquids or defects in solids, has the FWHM $\theta_{n}$ and an intensity $I_{n}$. The broad component, which is due to positron and Ps pick-off annihilations, is approximately Gaussian with a FWHM characterized by $\theta_{b}$ and accounts for the rest of the intensity.

\begin{tabular}{|c|c|c|c|c|c|}
\hline & $\begin{array}{c}\mathrm{T} \\
(\mathrm{K})\end{array}$ & $\stackrel{\theta_{n}}{(\operatorname{mrads})}$ & $\begin{array}{c}I_{n} \\
(\%)\end{array}$ & $\begin{array}{c}\theta_{b} \\
\text { (mrads) }\end{array}$ & Reference \\
\hline $\begin{array}{l}{ }^{4} \mathrm{He}(\mathrm{l}) \\
{ }^{4} \mathrm{He}(\mathrm{s})\end{array}$ & $\begin{array}{c}4.2 \\
1.7(1 \rightarrow 14 \mathrm{~atm}) \\
4.2(1 \rightarrow 100 \mathrm{~atm}) \\
1.7(50 \rightarrow 168 \mathrm{~atm}) \\
4.2(168 \mathrm{~atm})\end{array}$ & $\begin{array}{c}0.79 \pm 0.05 \\
1.00 \rightarrow 1.29 \\
0.89 \rightarrow 1.90 \\
1.52 \rightarrow 1.84 \\
2.13\end{array}$ & $5.0 \pm 0.5$ & 8.8 & $\begin{array}{c}{[43,276,44]} \\
{[291,130]} \\
{[291,130]} \\
{[291,130]} \\
{[291,130]}\end{array}$ \\
\hline $\mathrm{Ne}(\mathrm{l})$ & 27.1 & $1.7_{-0.1}^{+0.2}$ & $5.3 \pm 0.5$ & 12.6 & {$[43,44]$} \\
\hline $\begin{array}{l}\operatorname{Ar}(1) \\
\operatorname{Ar}(s)\end{array}$ & $\begin{array}{c}87.3 \\
85 \pm 1 \\
77 \\
83 \pm 1\end{array}$ & $\begin{array}{c}2.1_{-0.1}^{+0.2} \\
2.3\end{array}$ & $\begin{array}{c}7.4_{-0.7}^{+1.5} \\
6.8\end{array}$ & $\begin{array}{l}9.4 \\
9.4 \\
9.4 \\
9.4\end{array}$ & $\begin{array}{c}{[43,44]} \\
{[298]} \\
{[234]} \\
{[298]}\end{array}$ \\
\hline $\begin{array}{l}\mathrm{Kr}(\mathrm{l}) \\
\mathrm{Kr}(\mathrm{s})\end{array}$ & $\begin{array}{l}117 \pm 1 \\
115 \pm 1\end{array}$ & 2.6 & 8.2 & $\begin{array}{l}9.0 \\
9.0\end{array}$ & $\begin{array}{l}{[298]} \\
{[298]}\end{array}$ \\
\hline $\begin{array}{l}\mathrm{Xe}(1) \\
\mathrm{Xe}(\mathrm{s})\end{array}$ & $\begin{array}{l}162 \pm 1 \\
160 \pm 1\end{array}$ & 2.8 & 11.8 & $\begin{array}{l}8.0 \\
8.2\end{array}$ & $\begin{array}{l}{[298]} \\
{[298]}\end{array}$ \\
\hline $\mathrm{H}_{2}(\mathrm{l})$ & 20.4 & $1.3_{-0.1}^{+0.2}$ & $12 \pm 3$ & $6.6 \pm 0.2$ & {$[43,44]$} \\
\hline$N_{2}(l)$ & 77.3 & $1.8_{-0.1}^{+0.2}$ & $7.7_{-0.8}^{+1.5}$ & 8.8 & {$[43]$} \\
\hline $\mathrm{O}_{2}(\mathrm{l})$ & 90.2 & & & 10.1 & [43] \\
\hline
\end{tabular}


distribution similar to that described by (4.4). A ID-ACAR curve in metals therefore consists of an inverted parabola superimposed on top of a broad Gaussian component. When an electron-positron pair bind together as Ps the momentum of each particle is almost entirely canceled by that of the other. The resulting momentum distribution corresponds to the centre-of-mass motion of Ps and has a FWHM that ranges from a few tenths of a mrad for delocalized Ps to several mrads for Ps trapped in a cavity. In insulating media, the 1D-ACAR consists of a broad momentum component, due to free positron and Ps pick-off annihilations with the valence electrons, and a narrower component due to $p$-Ps self-annihilations (the $3 \gamma$ o-Ps self-annihilations cannot be observed). The relative area under each component $I_{i}$ gives a measure of the fraction of positrons that annihilate with that 'type' of electron.

Previous 1D-ACAR curves for the rare-gas liquids and solid $\mathrm{Ar}, \mathrm{Kr}$ and $\mathrm{Xe}$ are shown in Figure 4.2a. Figure 4.2b shows similar results for liquid $\mathrm{H}_{2}, \mathrm{~N}_{2}$, and $\mathrm{O}_{2}$ (Briscoe and Stewart 1967; Briscoe et al. 1968; Varlashkin 1971; and Stewart et al. 1990). As expected, each of the liquids consists of a broad momentum component and, except for $\mathrm{O}_{2}$, a narrow momentum component that is well described by the self-annihilation of $p$-Ps from inside a self-trapped 'bubble' state. The possible existence of other momentum components will be discussed later. Table 4.1 summarizes all the known experimental ID-ACAR results for these media previous to this work. More detailed descriptions of these results are given throughout the text.

\subsection{D-ACAR Results and Analysis}

In this thesis, 1D-ACAR experiments were performed in liquid $\mathrm{He}, \mathrm{Ne}, \mathrm{Ar}$ and $\mathrm{N}_{2}$ and in solid $\mathrm{Ne}$ and Ar. The magnetic quenching of Ps was studied in each of these condensed samples for the first time. External electric fields were also applied to liquid Ar and $\mathrm{N}_{2}$ samples in an effort to study the processes occurring in the positron spur. In another series of experiments, a known quantity of $\mathrm{O}_{2}$ impurity was deliberately mixed with liquid $\mathrm{Ar}$ and $\mathrm{N}_{2}$ samples to study its effects on Ps formation and conversion. Liquid Ar and $\mathrm{N}_{2}$ were chosen for these experiments so that the differences between mono- and diatomic liquids could be observed. 


\subsubsection{Data Corrections and Initial Analysis}

Data analysis began by applying several corrections to the experimental results. The first correction was the subtraction of random coincidence counts. True coincidences occur whenever both detectors are triggered by $\gamma$-rays from the same annihilation event. In addition to these true coincidences there are always false or random coincidences resulting from two completely unrelated $\gamma$-rays accidentally overlapping within the resolving time of the apparatus. Let $\tau_{1}$ and $\tau_{2}$ be the resolving time of the detectors and $N_{1}$ and $N_{2}$ the corresponding number of singles counts. The number of true coincidences is then $N^{\prime \prime}\left(\theta_{2}\right)=$ $N_{\text {ex }}\left(\theta_{z}\right)-N_{r}\left(\theta_{z}\right)$, where the number $N_{r}$ of random coincidence counts given by (Evans 1955)

$$
\begin{aligned}
N_{r}\left(\theta_{z}\right) & =\frac{\left(N_{1}\left(\theta_{z}\right)-N^{\prime \prime}\left(\theta_{z}\right)\right)\left(N_{2}\left(\theta_{z}\right)-N^{\prime \prime}\left(\theta_{z}\right)\right)\left(\tau_{1}+\tau_{2}\right)}{\Delta t} \\
& \approx \frac{2 \tau N_{1}\left(\theta_{z}\right) N_{2}\left(\theta_{z}\right)}{\Delta t}
\end{aligned}
$$

where the counting time $\Delta t$ is the same for each $\theta_{z}, \tau=\tau_{1}=\tau_{2}$ and $N^{\prime \prime} \ll N_{1}, N_{2} . N_{r}\left(\theta_{z}\right)$ was generally quite small - amounting to 1 to $2 \%$ of the counts at the ACAR peak.

A second correction was required to account for the relatively rapid decay of the ${ }^{64} \mathrm{Cu}$ positron sources. Each ACAR curve typically consisted of 3-10 scans over the set angular positions, with each scan taking from one to two hours to complete. The short 12.70 hour half life means that the source activity at the end of each scan was about 5-10\% lower than at the start. Both the singles and the coincidence counting rates are directly proportional to the source activity, which is $A \lambda=A_{0} \lambda e^{-\lambda t}$ at time $t$ with $A_{0} \lambda$ being the $t=0$ activity where $\lambda=\ln 2 /(12.70 \mathrm{hr})$ is the total radioactive decay constant. The ratio of activities at $t$ and $t=0$ is simply $e^{-\lambda t}$. To apply the correction a convenient $t=0$ reference point within each scan was chosen, usually close to when $\theta_{z}=0$. Since the counting times at each $\theta_{z}$ position are equal, the corrected number of coincidence counts is simply

$$
N^{\prime}\left(\theta_{z}\right)=N^{\prime \prime}\left(\theta_{2}\right) e^{\lambda t_{2}}
$$

where $t_{z}$ is the start of the counting interval at $\theta_{z}$ relative to $t=0$. Data from each scan of an experiment was corrected individually and then summed to give the corrected ACAR curve.

The initial analysis of the corrected ACAR data followed standard techniques. The functional form for each of the components in an ACAR curve is generally not known à 
Table 4.2: A summary of the parameters used to fit the broad momentum component of the experimental 1D-ACAR data. The broad component, which is due to Ps pick-off and positron annihilations, is largely independent of temperature, electric and magnetic fields and $\mathrm{O}_{2}$ concentration. It has been fit with using either one or two Gaussians with areas $A_{b 1}$ and $A_{b 2}$ and FWHM of $\theta_{b 1}^{\prime}$ and $\theta_{b 2}^{\prime}$. The area under the Gaussians has been normalized to 1 and has a FWHM given by $\theta_{b}^{\prime}$. Deconvolution with the resolution function gives a $\theta_{b}$ that is $0.01-0.02 \mathrm{mrads}$ narrower than $\theta_{b}^{\prime}$. Care should be taken in attaching any physical significance to the individual parameters $\theta_{b 1}^{\prime}, A_{b 1}, \theta_{b 2}^{\prime}$ and $A_{b 2}$ as many different combinations of these values result in a similar fit. However, it is expected that the sum of the Gaussians should give a reasonable first approximation to the broad component, thereby allowing a proper separation of the narrow p-Ps component. The second broad component estimate for liquid $\mathrm{N}_{2}$ was obtained in a completely arbitrary fashion. It has been normalized to an area of 1.0386 and results in narrow peak intensities that are about $3.5 \%$ lower than the best two Gaussian fit to the broad component.

\begin{tabular}{lccccc}
\hline & $\begin{array}{c}\theta_{b 1}^{\prime} \\
\text { (mrads) }\end{array}$ & $A_{b 1}$ & $\begin{array}{c}\theta_{b 2}^{\prime} \\
\text { (mrads) }\end{array}$ & $A_{b 2}$ & $\begin{array}{c}\theta_{b}^{\prime} \\
\text { (mrads) }\end{array}$ \\
\hline${ }^{4} \mathrm{He}(\mathrm{l})$ & 13.89 & 0.379 & 7.82 & 0.621 & 8.84 \\
$\mathrm{Ne}$ & 27.18 & 0.069 & 12.72 & 0.931 & 12.94 \\
$\mathrm{Ar}$ & 9.24 & 1.000 & & & 9.24 \\
$\mathrm{~N}_{2}(\mathrm{l})$ & 14.10 & 0.151 & 8.51 & 0.849 & 8.85 \\
& 9.77 & 0.874 & 4.79 & 0.165 & 7.92 \\
\hline
\end{tabular}

priori. It is therefore customary to model the data using reasonably simple functions with the aim of extracting physically meaningful parameters and allowing the various momentum components to be separated. In many non-metallic materials, each component in the experimental 1D-ACAR curve is often adequately fit by one or more Gaussians. The entire ACAR curve is usually then fit surprisingly well by the sum of two or three Gaussians

$$
N^{\prime}\left(\theta_{z}\right) \approx 2 \sqrt{\frac{\ln 2}{\pi}} \sum_{i} \frac{A_{i}}{\theta_{i}^{\prime}} \exp \left\{-4 \ln 2\left(\frac{\theta_{z}-\theta_{o}}{\theta_{i}^{\prime}}\right)^{2}\right\} .
$$

The fitting parameters are the FWHM of the Gaussians $\theta_{i}=2 \sqrt{2 \ln 2} \sigma_{i}$ with $\sigma_{i}$ being the standard deviation, the area $A_{i}$ under each component, and the centroid $\theta_{0}$. The relative intensity $I_{i}$ of a component is simply $A_{i}$ divided by the total area. The curve fitting was done using the nonlinear least-squares Levenberg-Marquardt technique (Press et al. 1992).

In most analysis, individual ACAR curves are fit separately with the parameters allowed to vary freely (Kirkegaard et al. 1989). The fitting procedure used here was somewhat different. As will be seen, changes in temperature, magnetic and electric fields, and $\mathrm{O}_{2}$ 
concentration have no measurable effect, on the shape of the broad momentum component. The shape of this component was therefore defined by a one or two Gaussian fit to the sum of many individual ACAR curves. The individual ACAR curves were then fit using the constraint that their broad components have this same shape. This process resulted in the best overall fit to the broad component and ensured that changes in the narrow component were always measured with respect to the same broad component. The relative areas and FWHM for the two Gaussian fits to the broad component for each sample are given in Table 4.2. Due to symmetry, the angular correlation curves that follow will be folded about the fitted centroid $\theta_{0}$ and so only positive angles will be shown. Furthermore, since the counting rate is uncalibrated the normalization of ACAR data is arbitrary. In these experiments, the total area under the broad component, as described by the Gaussian fits, was normalized to 1 .

The ACAR curves are smeared to some extent by the finite resolution of the apparatus and the by positron's motion. The measured ACAR curve $N^{\prime}\left(\theta_{z}\right)$ is, in general, a convolution of the unsmeared ACAR curve $N\left(\theta_{z}\right)$ and a resolution function $R\left(\theta_{z}\right)$ for the apparatus

$$
N^{\prime}\left(\theta_{z}\right)=\int_{-\infty}^{+\infty} N(x) R\left(\theta_{z}-x\right) d x
$$

Appendix $\mathrm{F}$ shows that the resolution function is well approximated by a unit area Gaussian function with a FWHM $\theta_{R}$ given by (F.2). In these experiments the apparatus was not used at its highest resolution ( $\sim 0.1 \mathrm{mrads}$ ). The slit widths were a compromise between counting rate and resolution. The detector slit widths $\theta_{w}$ were chosen so that $\theta_{R}$ would be small compared to the width of the features in the ACAR curve. For the $\mathrm{Ne}, \mathrm{Ar}$ and $\mathrm{N}_{2}$ samples the detector slit width was set to $6.35 \mathrm{~mm}$ so that $\theta_{w}=0.50 \mathrm{mrads}$. For He, which had narrower features, the slit widths were reduced to $3.18 \mathrm{~mm}$ which gave $\theta_{w}=0.25$ mrads. The collimating slit nearest the cryostat on the fixed detector side was set to $1.65 \mathrm{~mm}$ $\left(\theta_{t}=0.13 \mathrm{mrads}\right)$ and defined the sample thickness seen by the detectors. The collimating slit on the moving detector side was set a little larger, to $1.90 \mathrm{~mm}$, to minimize changes in the defined sample volume as the detector angle was varied. These slit widths resulted in unit area Gaussian resolution functions with FWHM's of 0.37 mrads for He, 0.58 mrads for $\mathrm{Ne}$, and 0.63 mrads for $\mathrm{Ar}$ and $\mathrm{N}_{2}$.

Given $R\left(\theta_{z}\right)$ it is possible, in principle, to deconvolute the experimental data to extract $N\left(\theta_{z}\right)$. However, the usual Fourier deconvolution techniques (Rotondi 1977; Press 
et al. 1992) are difficult to apply due to their sensitivity to noise in the data and the relatively small number of data points defining an ACAR curve. A simpler approach was therefore used. From the convolution theorem $\mathcal{F}\left[N^{\prime}\left(\theta_{z}\right)\right]=\mathcal{F}\left[N\left(\theta_{z}\right)\right] \mathcal{F}\left[R\left(\theta_{z}\right)\right]$ where $\mathcal{F}$ denotes the Fourier transform. $N\left(\theta_{z}\right)$ is therefore obtained by Fourier transforming (4.7), dividing by $\mathcal{F}\left[R\left(\theta_{z}\right)\right]$ and applying an inverse Fourier transform to the result. Since all the functions involved are Gaussians the process becomes trivial and can be done analytically. The result for $N\left(\theta_{z}\right)$ is similar to (4.7) except that now the FWHM for each Gaussian is $\theta_{i}=\sqrt{\left(\theta_{i}^{\prime}\right)^{2}-\theta_{R}^{2}}$. The only noticeable effect of the deconvolution is on the narrow component which increases in height by $5-10 \%$ and undergoes a corresponding reduction in FWHM.

\subsubsection{Experimental Results}

The data for liquid ${ }^{4} \mathrm{He}$ was collected using a single set of positron sources. During the useful lifetime of the sources it was possible to obtain four ACAR curves in the magnetic fields $B=0.0,0.20,0.40$, and $2.00 \pm 0.01 \mathrm{~T}$. The experiments were performed at $4.6 \pm 0.2 \mathrm{~K}$ and at vapour pressures of about 1100 torr. Lower temperatures could not be easily obtained due to thermal conduction down the support structure of the cryostat insert. The corrected (but not deconvoluted) data for each of the magnetic field values are shown in Figure 4.3. The small width of the narrow peak makes fitting the broad component relatively simple and accurate. Its shape is well described over the entire range of angles using the Gaussian parameters in Table 4.2 and shows no measurable dependence with magnetic field. The helium broad component measured by Briscoe et al. (1968), shown in Figure 4.2, was also well fit using these parameters. In both cases, the experimental broad component has a FWHM of $8.8 \pm 0.2$ mrads. One Gaussian fits to the broad component are significantly poorer - typically too low in the 0 to 2.0 and 9.5 to $15 \mathrm{mrad}$ region and too high in the 2.5 to $8.0 \mathrm{mrad}$ region.

Using the fitted broad component it is possible to separate out the narrow component. The intensity $I_{n}$ and FWHM $\theta_{n}$ of this component was determined by a single Gaussian fit superimposed on top of the broad component. The average $\theta_{n}$ is $0.88 \pm 0.05$ mrads which is reduced to $0.79 \pm 0.05$ mrads after deconvolution with the experimental resolution function. This result is identical to that measured by Briscoe et al. (1968). The narrow peak FWHM showed no measurable dependence on magnetic field. The change in $I_{n}$ as a function of 


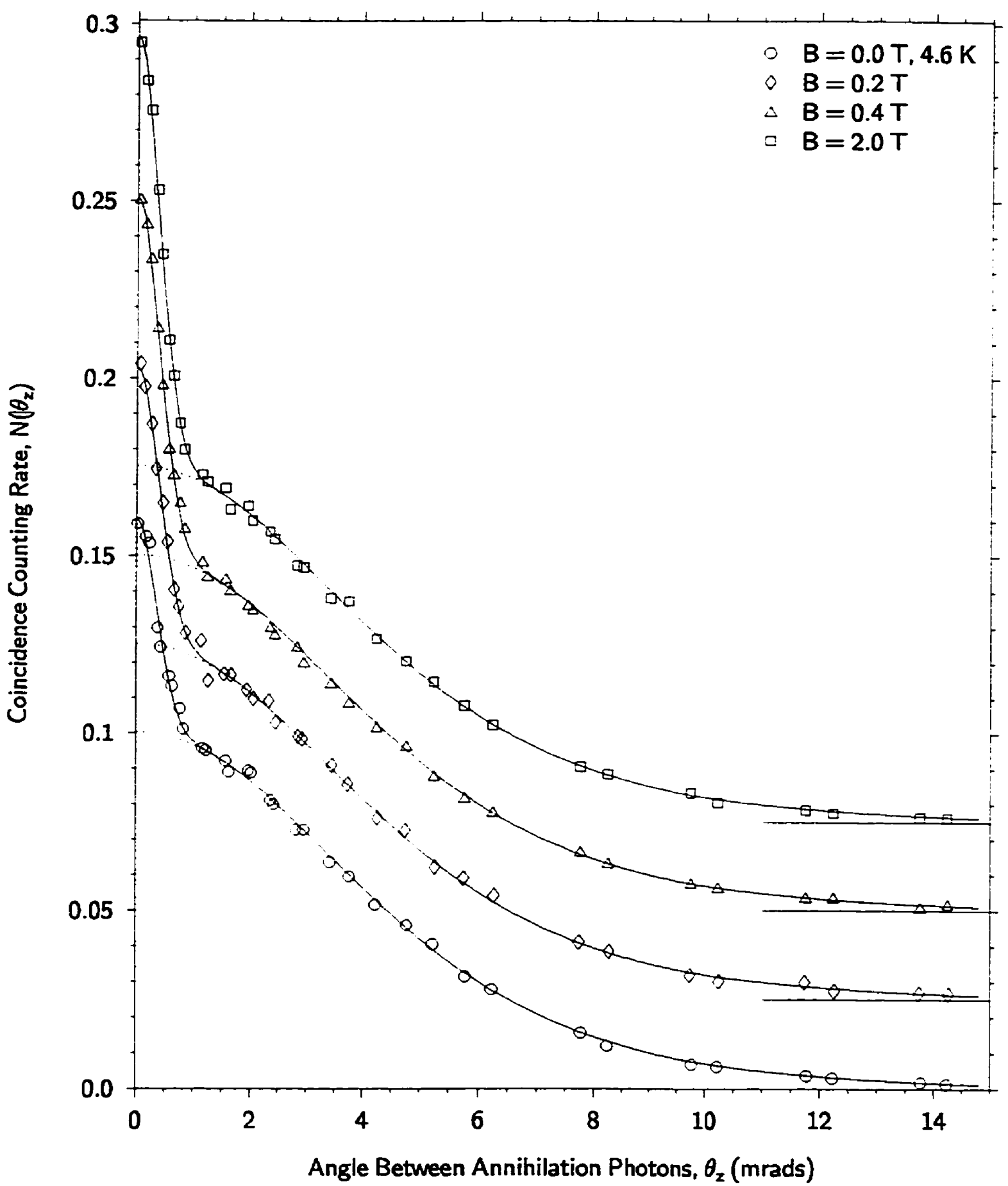

Figure 4.3: Experimental ACAR data for positrons annihilating in liquid helium at $4.6 \pm 0.2 \mathrm{~K}$ and in magnetic fields of $0.0,0.20,0.40$ and $2.00 \pm 0.01 \mathrm{~T}$. The solid lines correspond to the three Gaussian fits to the data. The dotted line shows the position of the broad momentum component underneath the narrow component. The parameters for the two Gaussians fit to the broad component of each ACAR curve are given in Table 4.2. 


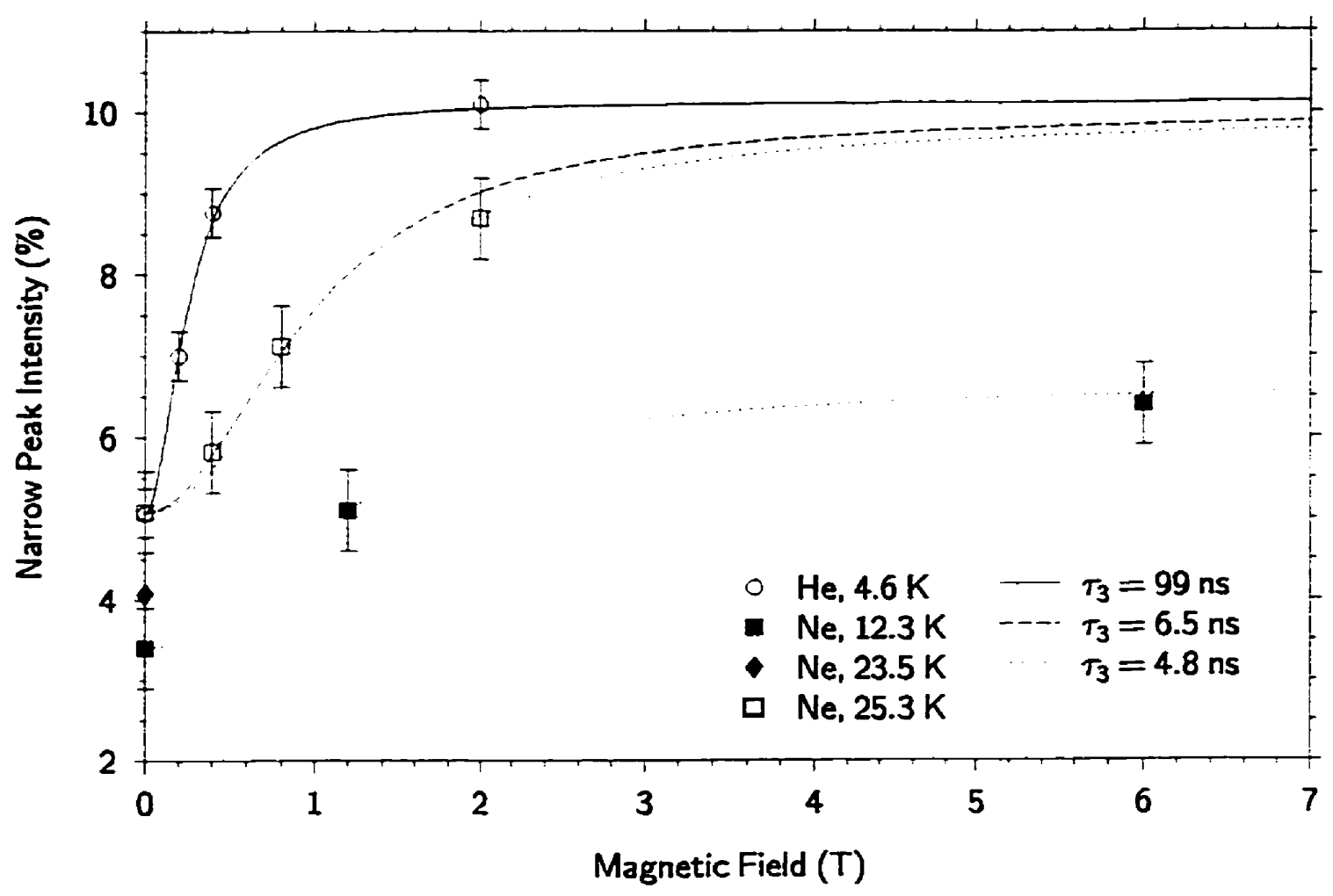

Figure 4.4: The intensity of the narrow peak as a function of magnetic field in liquid helium and in liquid and solid neon. The lines correspond to the theoretical predictions of (3.56) which describe the increase in the narrow peak intensity with magnetic field assuming no other Ps quenching processes are present. Equation 3.60 gives identical results. The required pick-off annihilation rate is obtained from the experimental o-Ps lifetimes, $\tau_{3}$. For liquid neon, where no lifetime experiments have been performed, the best fit to the data predicts a lifetime of $\tau_{3}=6.5 \pm 2 \mathrm{~ns}$.

magnetic field is shown in Figure 4.4. The zero-field narrow peak intensity compares well with the Briscoe et al. (1968) result (see Table 4.1) and with lifetime experiments which have consistently measured an $o$-Ps intensity of about $15 \%$ (at $B=0 I_{n}$ should be one-third of $I_{3}$ assuming no Ps spin-conversion mechanisms are present). The number of coincidence counts at the peak ranged from 3000-8000 giving a statistical accuracy of about $1-2 \%$. The uncertainty in the intensities and widths reflect the rather mediocre counting statistics, the small number of points defining the narrow component and the uncertainty in the quality of the broad component fit. The solid line in Figure 4.4 is the theoretical prediction of (3.56) obtained using the experimental $o$-Ps lifetime of $\tau_{3}=99$ ns to deduce the pick-off annihilation rate. Equation (3.60) gives a nearly identical prediction. The magnetic field results are discussed in detail in $§ 6.4$. 


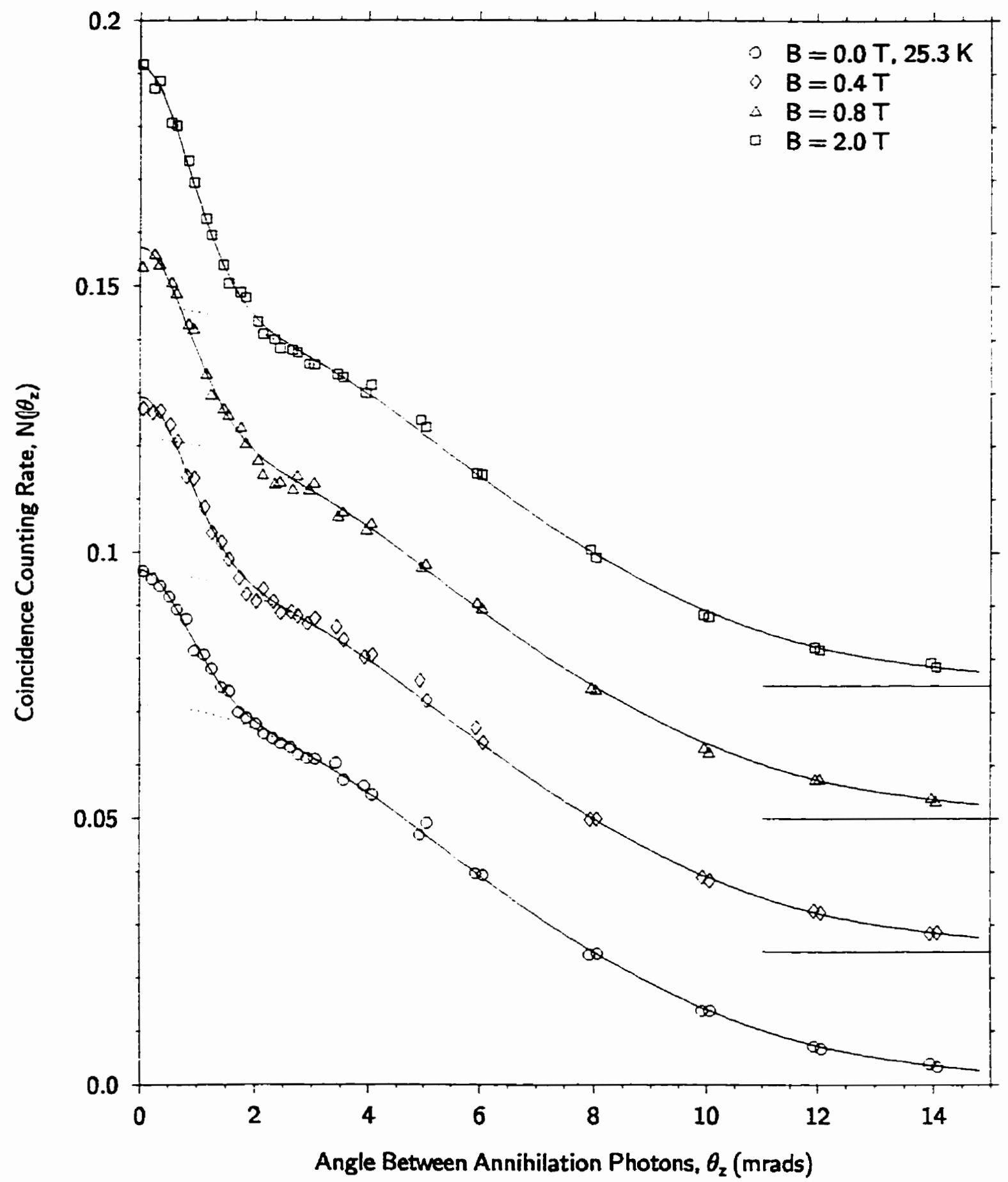

Figure 4.5: The corrected but not deconvoluted 1D-ACAR data for positrons annihilating in liquid neon at $25.3 \pm 0.1 \mathrm{~K}$ and in magnetic fields of $0.0,0.40,0.80$ and $2.00 \pm 0.01 \mathrm{~T}$. The solid lines correspond to the three Gaussian fits to the data. The dotted line shows the position of the best average Gaussian fit to the broad momentum component underneath the narrow component. The Gaussian parameters are given in Table 4.2. 


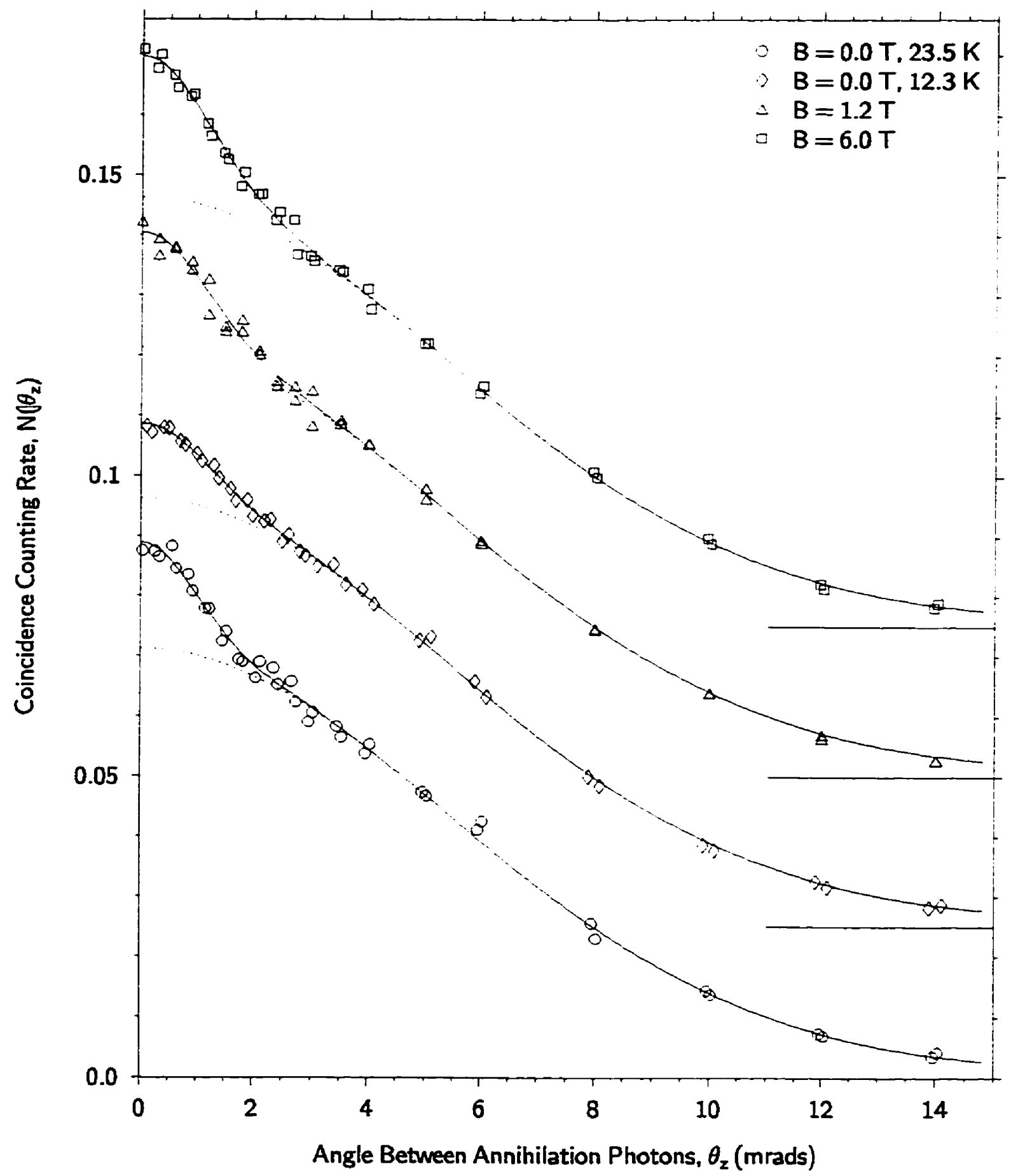

Figure 4.6: The corrected but not deconvoluted 1D-ACAR data for positrons annihilating in solid neon at $12.3 \pm 0.1 \mathrm{~K}$ and in magnetic fields of $0.0,1.20$ and $6.00 \pm 0.01 \mathrm{~T}$. A second $B=0 \mathrm{~T}$ ACAR curve in a sample at one degree lower than the melting temperature is also shown. The solid lines once again correspond to three Gaussian fits to the data which use the same broad component as was used for the liquid data. 
The data in condensed neon was collected using three sets of ${ }^{64} \mathrm{Cu}$ sources which provided four ACAR curves in both solid and liquid neon at various magnetic field values. The results are shown in Figures 4.5 and 4.6. The data typically had 3000-9000 counts at the peak which again implies a statistical accuracy of $1-2 \%$. The large difference in the broad and narrow peak widths allows the broad component to be separated with some confidence, though the process is less certain than in liquid helium. The broad component shape in both liquid and solid is again well described by a two Gaussian fit using the parameters in Table 4.2. The FWHM of $12.9 \pm 0.2$ mrads again shows no measurable dependence with magnetic field. These same parameters also provide a good fit to the liquid neon broad component of Briscoe et al. (1968).

Using this fit to represent the broad component gives narrow peaks in liquid neon with a FWHM of $1.95 \pm 0.07$ mrads, which is reduced to $1.86 \pm 0.07$ mrads when deconvoluted with the $0.58 \mathrm{mrad}$ wide resolution function. To date, there has been no lifetime measurements in liquid neon. The best fit of (3.56) to the data predicts an o-Ps lifetime of $\tau_{3}=6.5 \pm 2 \mathrm{~ns}$. Obviously, several more experiments would have been useful to define better the $I_{n}$ versus $B$ curves.

The narrow peaks in solid neon are significantly wider than in the liquid. The zero magnetic field experiment at $23.5 \mathrm{~K}$ had a deconvoluted narrow peak FWHM of $2.18 \pm$ 0.10 mrads and an intensity of $4.1 \pm 0.5 \%$. The three experiments at $12.3 \mathrm{~K}$ had an average deconvoluted narrow peak FWHM of $2.60 \pm 0.07$ mrads. The narrow peak intensities as a function of magnetic field are shown in Figure 4.4. The increase in $I_{n}$ with field in solid neon is again well described using (3.56) with an $o$-Ps lifetime of $\tau_{3}=4.8 \mathrm{~ns}$ which is consistent with the lifetime results in Table 4.3. The $B=0$ intensities are somewhat larger than expected from the measured lifetime intensities. This is most likely a consequence of differences in the solid growing conditions resulting in different defect concentrations and sizes.

The ACAR data in condensed argon was collected using seven sets of positron sources. Typical results for positrons annihilating in a magnetic field in liquid and solid argon are shown in Figures 4.7 and 4.8, respectively. As before, the statistical accuracy of this data is in the $1-2 \%$ range. Unlike helium and neon, the width of the broad and narrow components in argon makes it difficult to accurately separate these peaks. The dotted line in Figures 4.7 and 4.8 is the best one Gaussian fit to the broad component (two Gaussian fits 


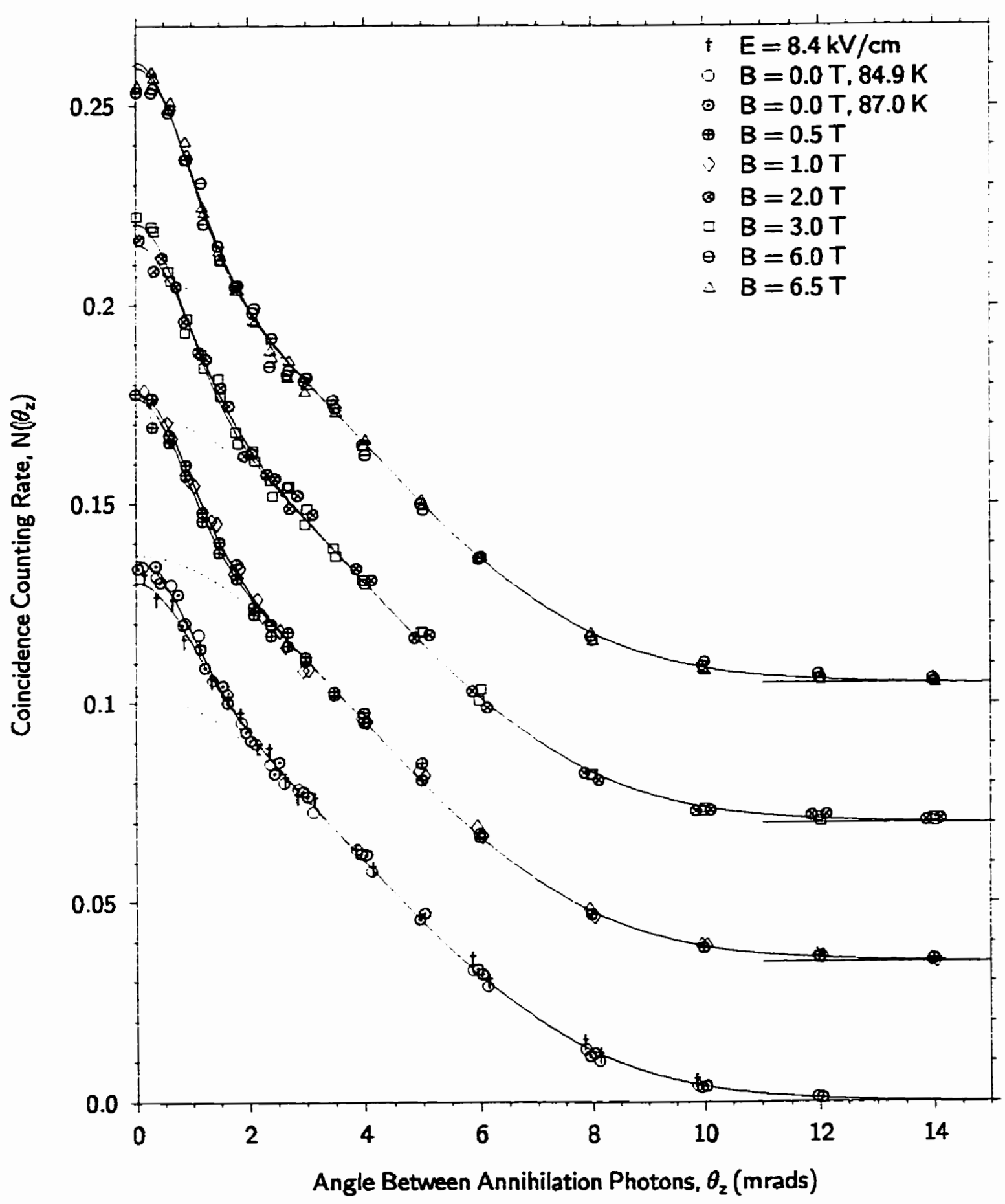

Figure 4.7: The corrected but not deconvoluted 1D-ACAR data for positrons annihilating in liquid argon at temperatures between 84.2 and $87.0 \mathrm{~K}$ and in various static electric and magnetic fields. The lines correspond to the two Gaussian fits to the data. 


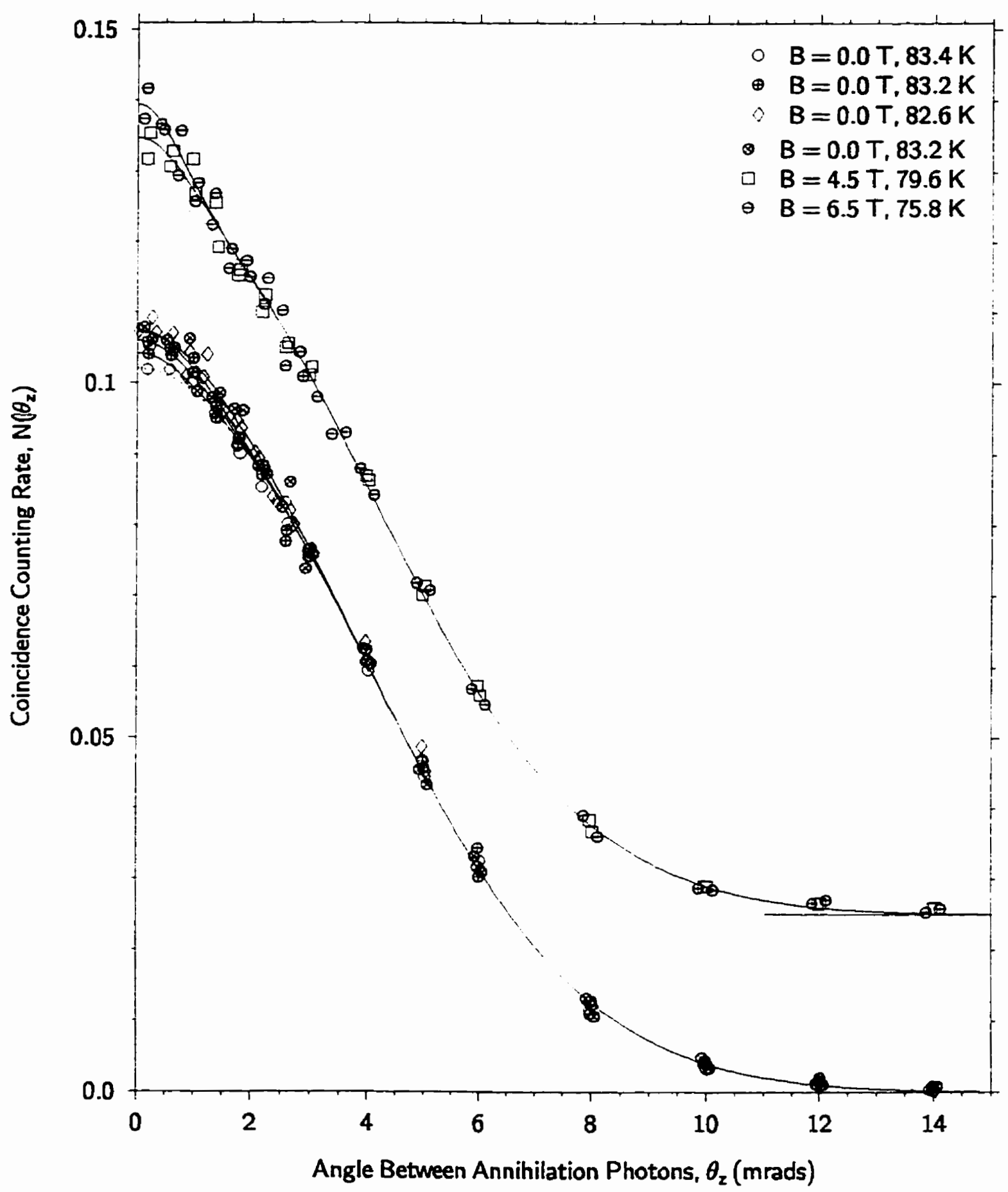

Figure 4.8: Typical 1D-ACAR data for positrons annihilating in solid argon at zero and high magnetic fields. The one Gaussian fit to the broad component is the same as was used to analyze the liquid argon data. 


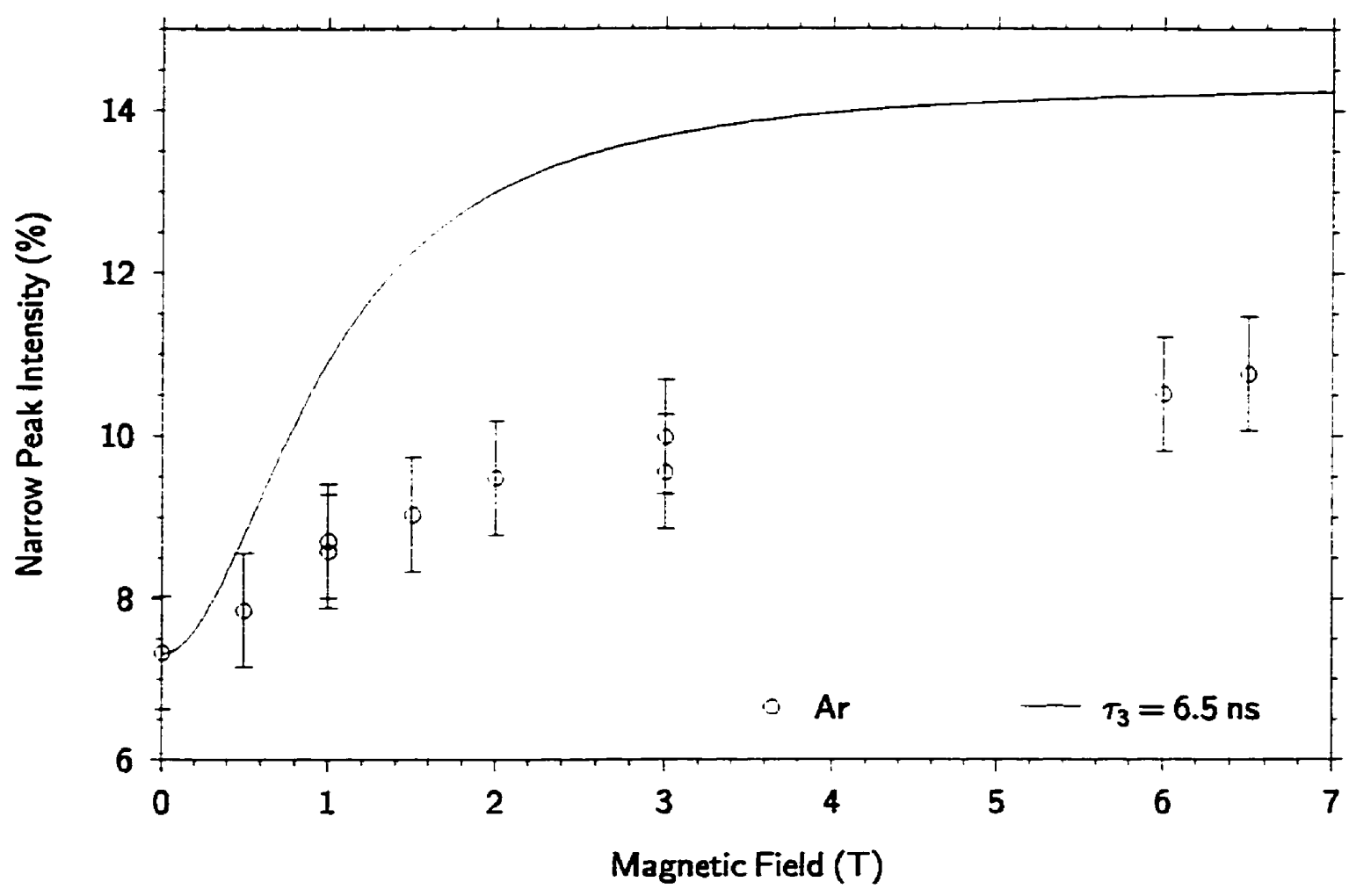

Figure 4.9: The intensity of the narrow peak as a function of magnetic field in liquid argon. The solid line correspords to the theoretical predictions of (3.56) or (3.60) using a pick-off annihilation rate obtained from the experimental o-Ps lifetime of $\tau_{3}=$ $6.50 \pm 0.06$. These predictions assume that no other quenching mechanisms are present, which is obviously incorrect.

give nearly identical results) and predicts a FWHM of $9.2_{-0.1}^{+0.3}$ mrads. This result is in good agreement with previous estimates by Rose and DeBenedetti (1965), Briscoe et al. (1968) and Varlashkin (1971). However, it is difficult to know how well this fit actually represents the broad component. Some guidance is provided by the solid argon data which gives an upper limit to the height of the broad component. Both Rose and DeBenedetti (1965) and Varlashkin (1971) assumed that no Ps was formed in their solid argon samples. If this is correct, then their ACAR curves correspond to the broad component. The shape of these curves are well described by our one Gaussian fit over the entire range of angles. The solid data in Figure 4.8 typically has a small component extending above the assumed broad component which is attributed to Ps annihilating from inside defects. As with neon, the solid growing conditions likely introduced numerous defects with sufficient free volume for Ps to form at or trap into and eventually annihilate from. 
This estimate of the broad component results in a narrow peak FWHM in liquid argon of $2.2_{-0.1}^{+0.2} \mathrm{mrads}$, which becomes $2.1_{-0.1}^{+0.2}$ mrads when deconvoluted with the $0.63 \mathrm{mrad}$ Gaussian resolution function. The zero magnetic field narrow peak intensity of $7.3 \pm 0.5 \%$ compares well with the result of Briscoe et al. (1968) but is somewhat larger than the $6.8 \%$ measured by Varlashkin (1971). This result is in serious disagreement with lifetime measurements which measure a long-lived $o$-Ps intensity $I_{3}$ of only $7.0 \pm 0.2 \%$. The obvious implication is that other quenching mechanisms are present in liquid argon that were not present in liquid helium or neon. This conclusion is strengthened even further from the plot of narrow peak intensity as a function of magnetic field shown in Figure 4.9. In this case, the narrow peak intensities fall well short of the prediction given by (3.56), which assumes that the magnetic field is the only available quenching mechanism. The other likely quenching mechanisms that could be present are spin-exchange conversions on small concentrations of impurities, such as $\mathrm{O}_{2}$, or on various spur species, such as the secondary electrons.

To study the $\mathrm{O}_{2}$ spin-exchange process further, a series of experiments were performed in which small quantites of $\mathrm{O}_{2}$ impurity were deliberately added to liquid argon. Typical ACAR curves are shown in Figure 4.10. The broad component has been fit using the same Gaussian curve as before. The addition of $\mathrm{O}_{2}$ had no measurable effect the on FWHM of the narrow peak. However, it does have a dramatic effect on the narrow peak intensity, as shown in Figure 4.11. Several more experiments between 0.2 and $0.8 \% \mathrm{O}_{2}$ impurity should have been done to better define this curve's peak.

An external electric field was applied to the liquid argon sample in two experiments. The aim of these experiments was to separate the positron from the spur electrons to study the effects on Ps spur formation and the possibility of Ps spin-exchange conversions with spur electrons. Figure 4.7 a shows an ACAR curve for liquid argon in an $8.4 \pm 0.4 \mathrm{kV} / \mathrm{cm}$ electric field. Higher electric fields resulted in dielectric breakdown. The narrow peak intensity, given in Figure 4.16, shows a slight decrease in intensity with electric field due to either reduced spur Ps formation or reduced spin-exchange conversions with spur electrons.

The narrow peaks measured in solid argon are difficult to analyze in a meaningful way. The FWHM of the narrow peak over many experiments ranges from 1.6 to 3.5 mrads while the zero magnetic field intensity typically varies from 0.3 to about $2 \%$. In large magnetic fields, the narrow peak intensity is usually somewhat higher, typically $1.5-2.5 \%$. This increase shows that small amounts of Ps are present in these samples. The large variation in 


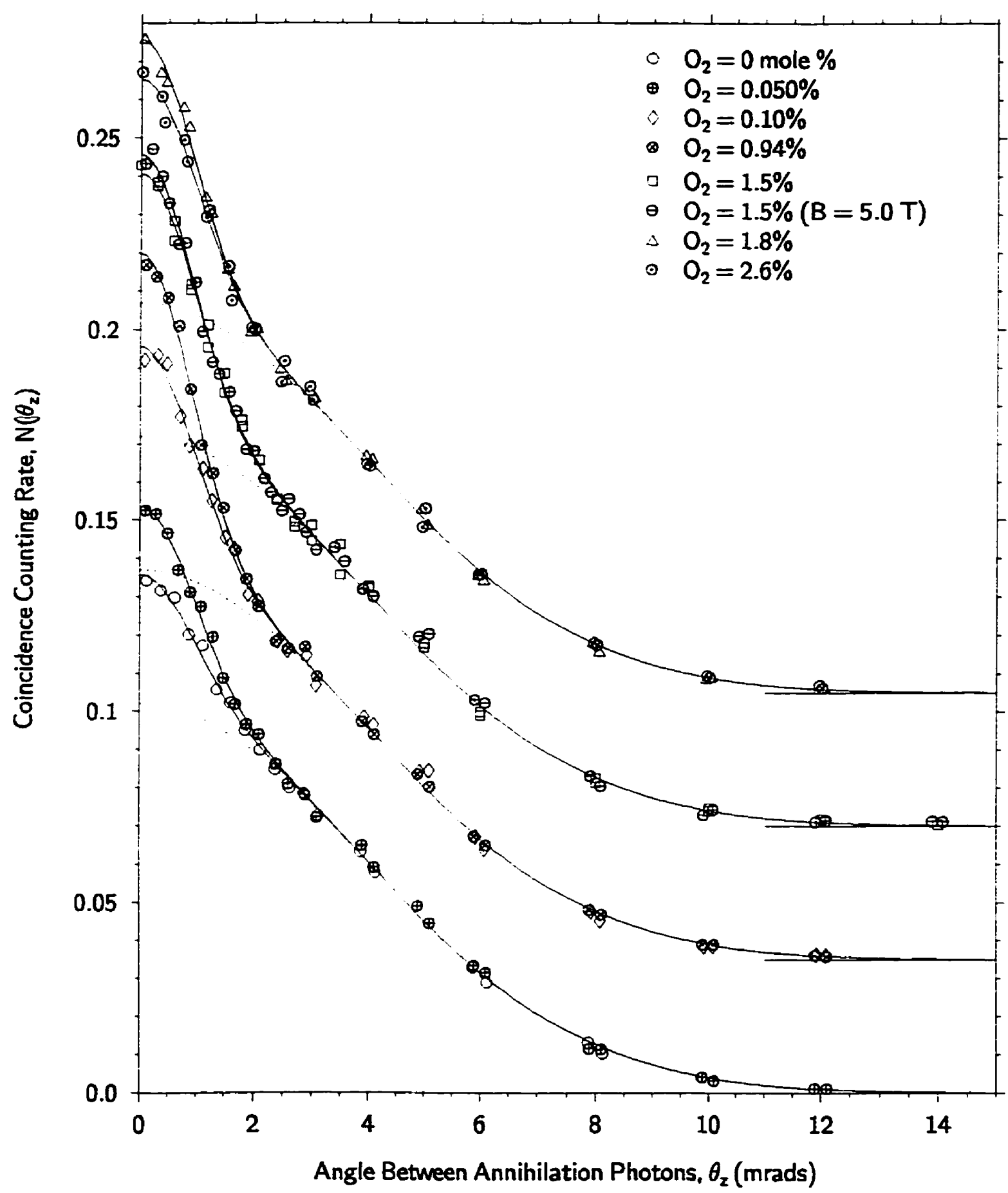

Figure 4.10: Typical 1D-ACAR data for positrons annihilating in liquid argon with small quantities of $\mathrm{O}_{2}$ impurities deliberately added. The $\mathrm{O}_{2}$ concentration was calculated assuming all of the $\mathrm{O}_{2}$ dissolved in the liquid argon sample. The fitted Gaussian broad component is the same as was used to analyze all the other argon data. 


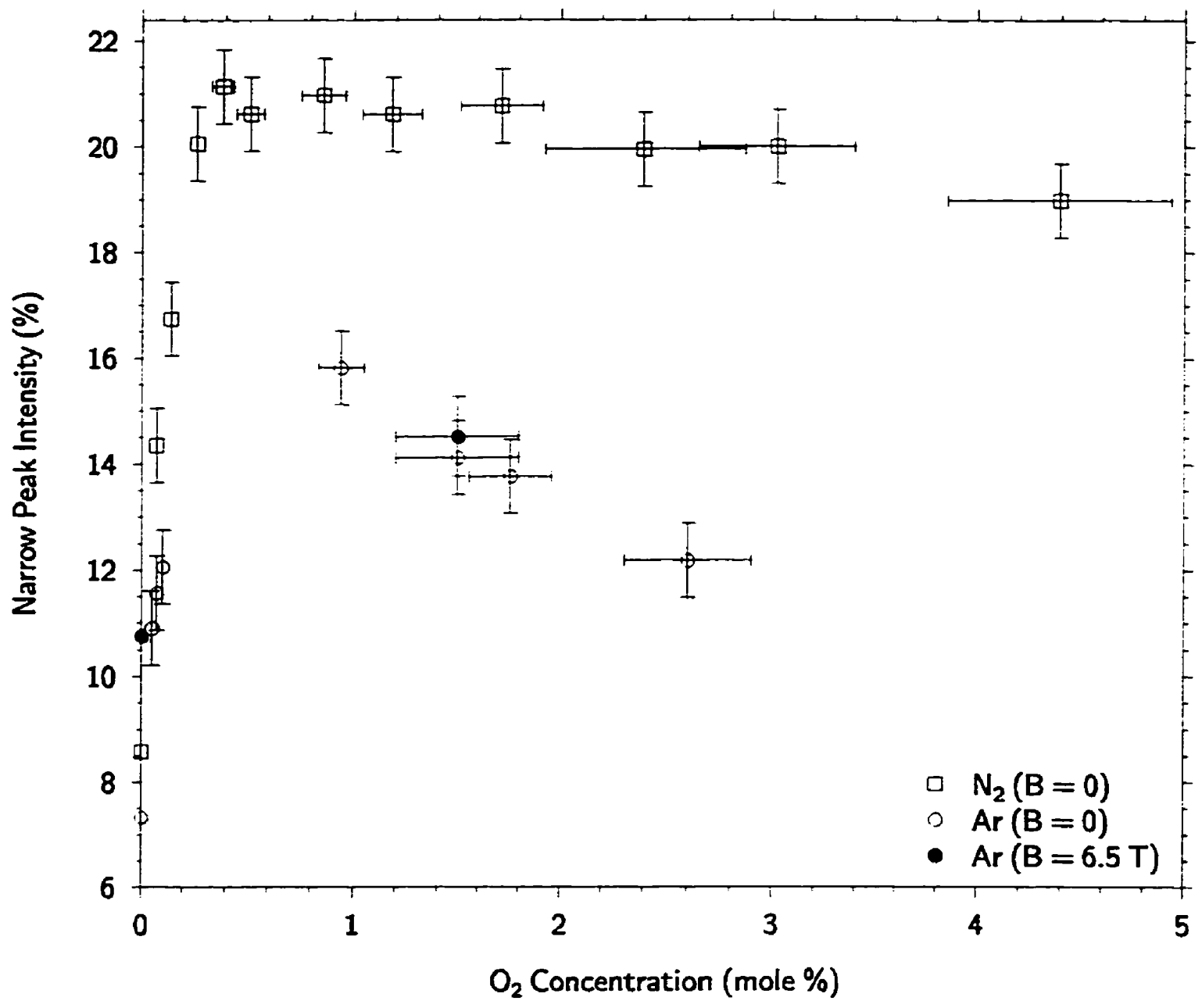

Figure 4.11: The narrow peak intensity as a function of $\mathrm{O}_{2}$ impurity concentration in liquid argon (circles) and nitrogen (squares). The two open circles correspond to measurements in argon at 0 and $1.5 \% \mathrm{O}_{2}$ in a magnetic field of $6.5 \mathrm{~T}$.

narrow peak FWHM and intensity implies that the Ps formation fraction and annihilation characteristics are very sensitive to growing conditions of the solid sample, which affects both the concentration and size of the free volume defects. Unlike the solid neon data, these ACAR curves were obtained in different solid samples, each having somewhat different defect concentrations and sizes. Furthermore, the narrow peak intensities are small compared to the uncertainties in the data making it difficult to extract useful information.

Seven sets of positron sources were used to measure the ACAR curves in liquid nitrogen as a function of electric and magnetic fields and $\mathrm{O}_{2}$ concentration. Typical results for positrons annihilating in various magnetic fields are shown in Figure 4.12. As with argon, it is difficult to accurately separate the broad and narrow components. The dotted line again corresponds to the best two Gaussian fit to the broad component shape. The 


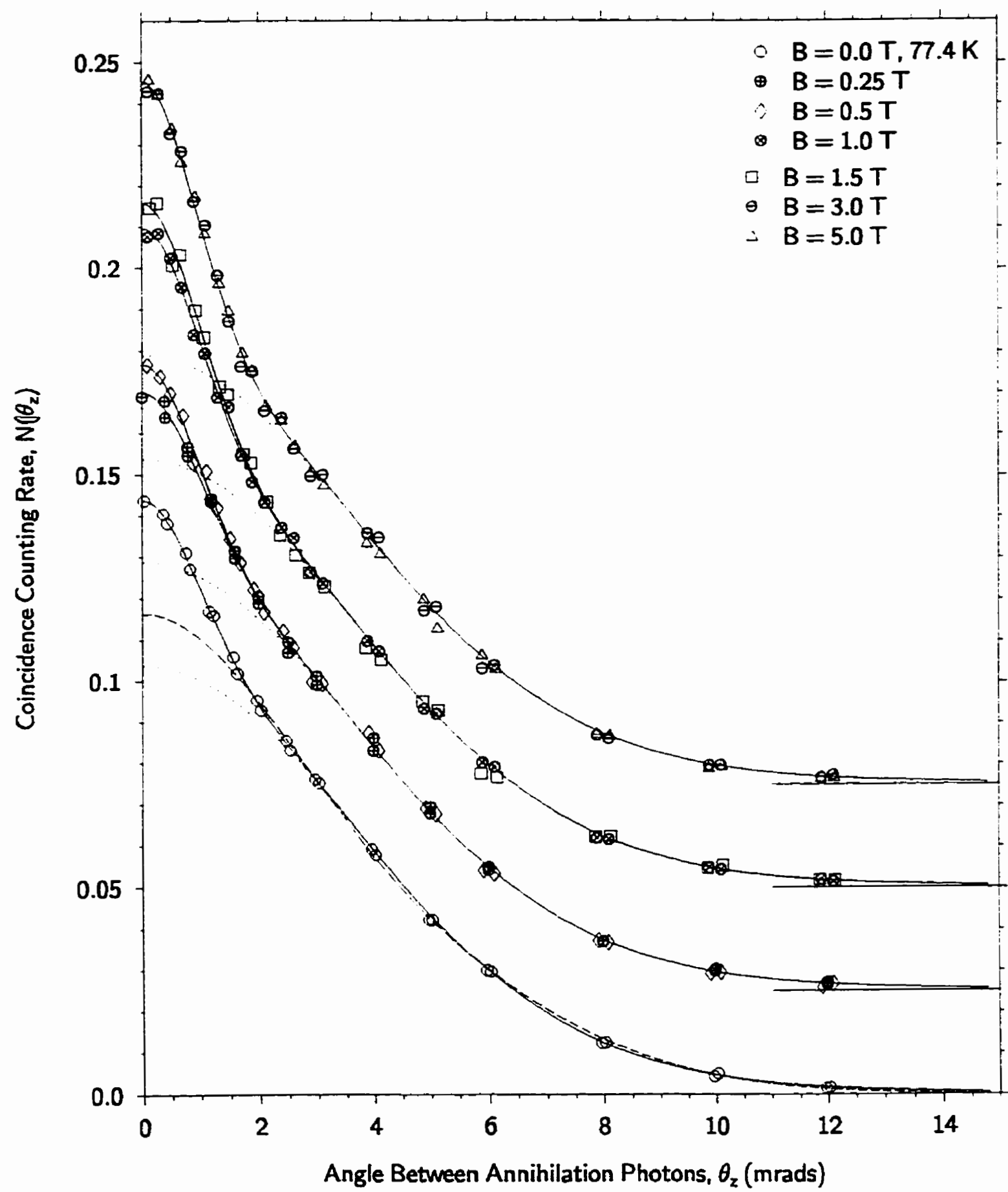

Figure 4.12: The corrected but not deconvoluted 1D-ACAR data for positrons annihilating in liquid nitrogen at $77.4 \mathrm{~K}$ and at various magnetic field values. The dotted line corresponds to the best two Gaussian fit to the broad component. A second estimate to the broad component is given by the dashed line. The area under this curve has been normalized to 1.0386 , which corresponds to a reduction of about $3.5 \%$ in the narrow peak intensity. 
fitting parameters are given in Table 4.2. This curve predicts a broad component FWHM of 8.85 mrads. As with the other samples, this component shows no measurable dependence on the magnetic field. The broad component shape is in good agreement with the only known measurement by Briscoe et al. (1968). However, it is again difficult to know if this fit is an accurate representation of the broad component. Furthermore, there have been no measurements in solid nitrogen which would provide an upper limit on the peakedness of the broad component. Briscoe et al. (1968) performed one experiment in impure liquid nitrogen which appears to have no narrow component and which is described very nicely by the two Gaussian broad component fit. However, it is unclear what was actually measured in their experiment, making it difficult to attach much importance to this result. To demonstrate the sensitivity of the results and their interpretation to the choice of broad component, a second more peaked broad component has also been fit to the data and is shown as the dashed line in Figure 4.12. The parameters for this curve were obtained in a completely arbitrary fashion. This choice of broad component, however, has the virtue that the resulting narrow peak intensities follow the behaviour expected in both electric and magnetic fields when no other spin-exchange conversion mechanisms are present. Angular correlation experiments in solid $\mathrm{N}_{2}$ experiments would be useful to obtain an upper limit on the broad component.

Using the best two Gaussian fit to the broad component results in a deconvoluted narrow peak FWHM of $2.15 \pm 0.20$ mrads. The zero-field narrow peak intensity is $8.6 \pm 0.7 \%$, which is in good agreement with the Briscoe et al. (1968) result when it is fit using the same broad component. Positron lifetime experiments measure an 0 -Ps intensity of about $15 \%$ which once again suggests that $\mathrm{Ps}$ spin-exchange conversion processes are present in liquid nitrogen. Figure $4.13 \mathrm{a}$ shows the narrow peak intensity as function of magnetic field again falling short of what would be predicted assuming the magnetic field was the only quenching mechanism present. The second, more peaked estimate for the broad component gives a deconvoluted narrow peak FWHM of $1.9_{-0.1}^{+0.20}$ mrads. The zero-field narrow peak intensity drops to $5.1 \%$, which is close to the prediction of lifetime experiments. Furthermore, the narrow peak intensity now follows the predicted dependence with magnetic field, as shown in Figure 4.13b. These results imply that no other Ps spin-conversion processes are present - a very different conclusion than was obtained using the best two Gaussian fit to the broad component.

A series of experiments were performed in which small amounts of $\mathrm{O}_{2}$ impurity were deliberately added to liquid nitrogen. Typical ACAR curves are shown in Figure 4.14. 


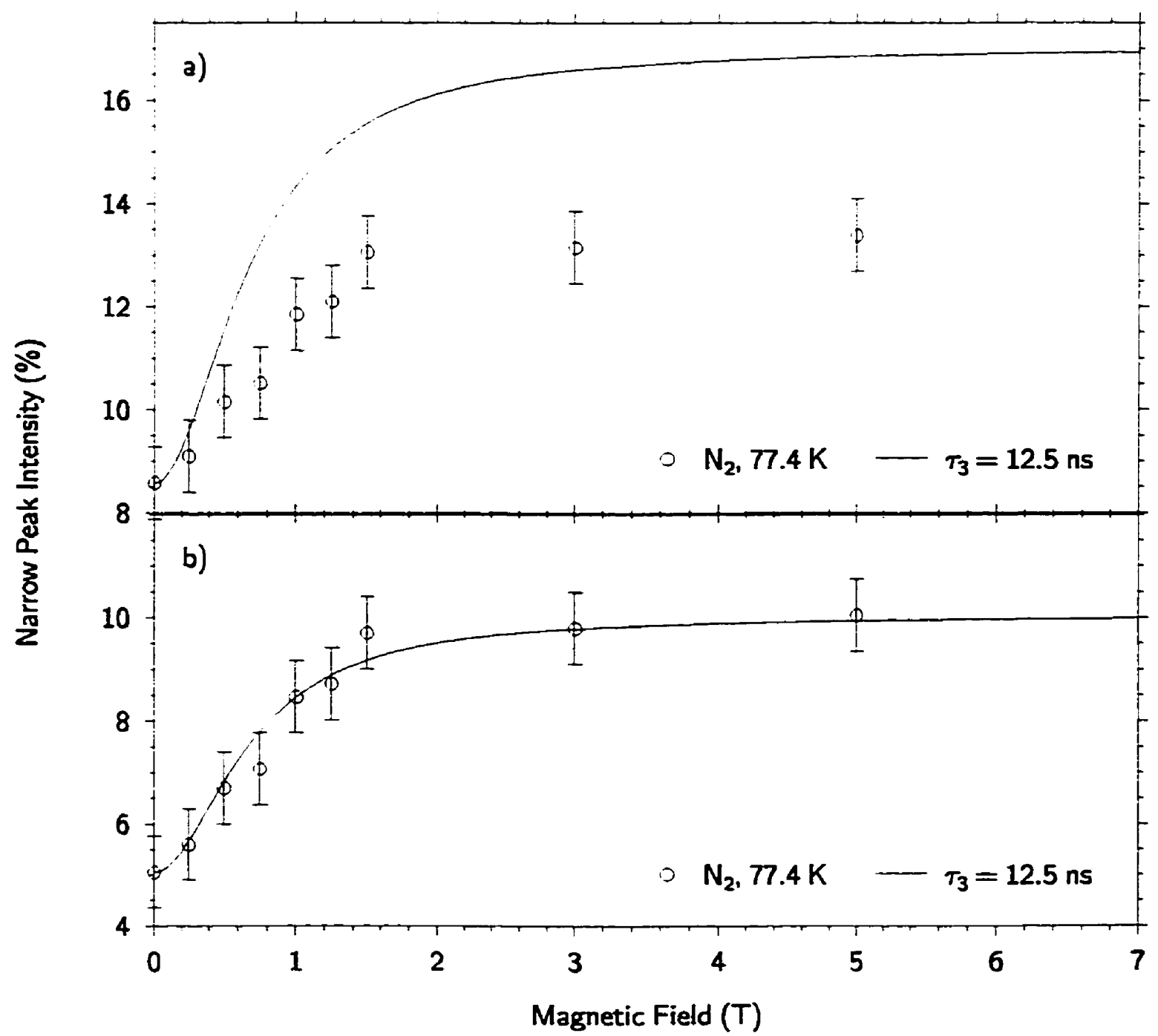

Figure 4.13: The intensity of the narrow peak as a function of magnetic field in liquid nitrogen assuming a) that the best two Gaussian fit describes the broad component or $b$ ) that the more peaked curve is a better estimate of the broad component. The data is the same in both cases. The solid lines correspond to the predictions of (3.56) and (3.60) using a pick-off annihilation rate obtained from the experimental $o$-Ps lifetime of $\tau_{3}=12.5 \pm 0.2$. These predictions assume no other quenching mechanisms are present. 


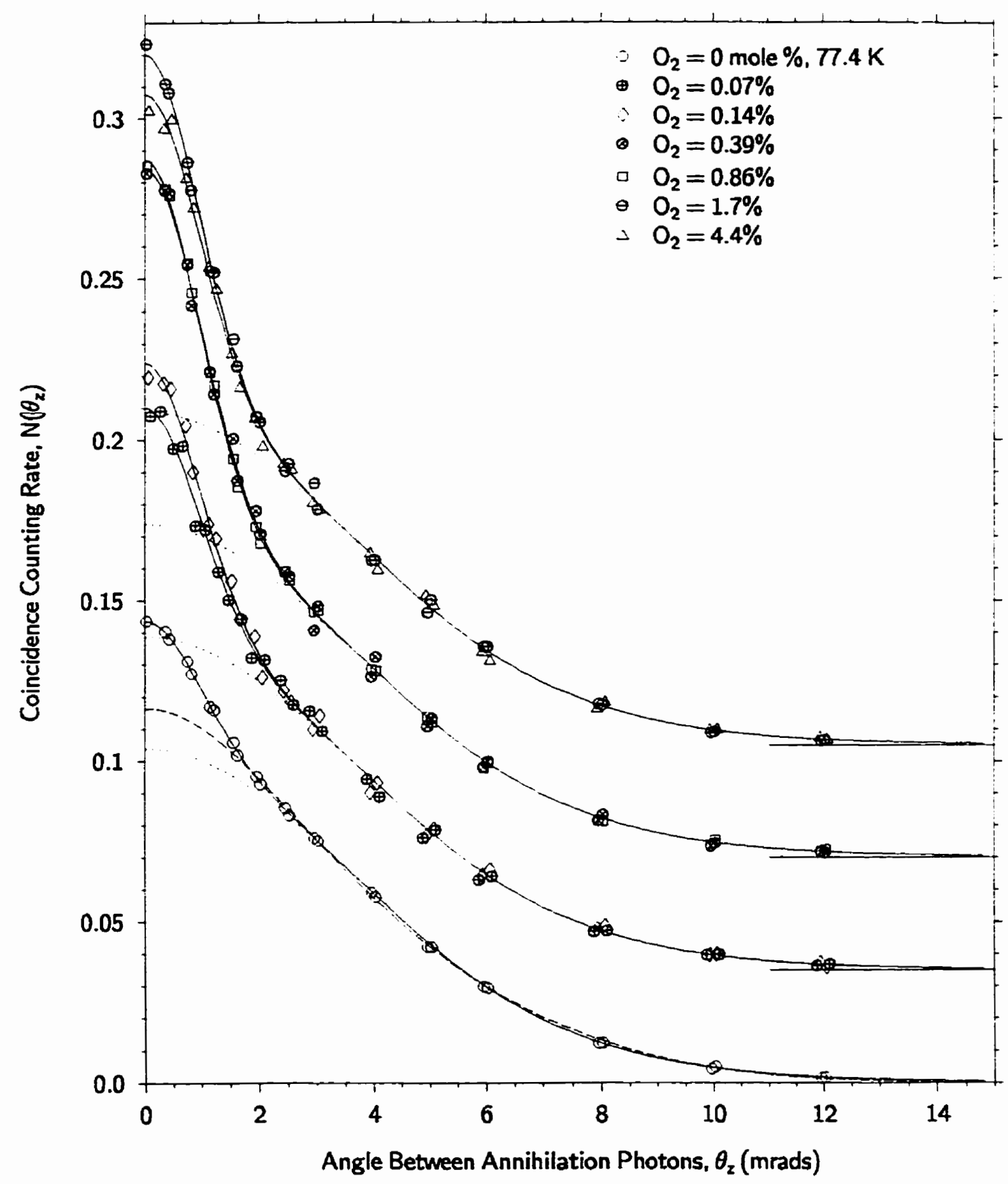

Figure 4.14: Typical 1D-ACAR data for positrons annihilating in liquid nitrogen with small quantities of $\mathrm{O}_{2}$ impurities deliberately added. The $\mathrm{O}_{2}$ concentration was calculated assuming all of the $\mathrm{O}_{2}$ dissolved in the liquid nitrogen sample. The dotted line corresponds to the best two Gaussian broad component. The dashed line is an arbitrary Gaussian fit that gives the expected electric and magnetic field dependence of $I_{n}$ assuming no Ps spin-conversion processes are present. 
The $\mathrm{O}_{2}$ impurity had no measurable effect on the FWHM of either the broad or narrow component. Figure 4.11 gives the narrow peak intensity as a function of $\mathrm{O}_{2}$ concentration using the best two Gaussian fit to estimate the broad component. The more peaked broad component estimate results in narrow peak intensities that are about $3.5 \%$ lower. In either case, $I_{n}$ shows a dramatic increase at small $\mathrm{O}_{2}$ impurity concentrations, peaking at around $21 \%$ at 0.4 mole $\% \mathrm{O}_{2}$, and then has a linear slowly decreasing behaviour.

In a final series of experiments various electric field strengths were applied to the liquid nitrogen samples. Typical ACAR data is shown in Figure 4.15. Once again the width of the various components is unaffected by the field. However, as with argon, $I_{n}$ decreases with increasing field strength. The results using the best Gaussian fit are summarized in Figure $4.16 \mathrm{a}$. Figure $4.16 \mathrm{~b}$ shows the same data with the only difference being that the more peaked broad component was used to determine the narrow peak intensity. Pepe et al. (1993) performed lifetime measurements in liquid nitrogen with an electric field applied. Using their measured o-Ps intensities, and assuming no spin-conversion mechanisms are present, gives the narrow peak intensities shown in Figure 4.16. The excellent agreement suggests that the more peaked curve may be a better estimate of the actual broad component.

\subsection{Positron Annihilation Lifetime Spectroscopy}

PAL spectroscopy measures the time interval between a prompt $\gamma$-ray, which signals the birth of a positron, and one of the $0.511 \mathrm{MeV}$ annihilation $\gamma$-rays. Lifetime experiments commonly use ${ }^{22} \mathrm{Na}$ as their positron source. As shown in Figure B.la, the emission of a positron transforms ${ }^{22} \mathrm{Na}$ into an excited ${ }^{22} \mathrm{Ne}$ which rapidly ( $<5 \mathrm{ps}$ ) decays to the ground state by emitting a $1.28 \mathrm{MeV} \gamma$-ray. It is this $\gamma$-ray that is used to signal the positron's birth. ${ }^{22} \mathrm{Na}$ is also convenient since a high fraction of the decays give positrons, reducing the background counts, and because of its long half-life (see Table B.2). Once in the sample, the positron's lifetime depends on the particular state that it annihilates from. Typical measurable lifetimes are $\sim 0.12$ ns for $p$-Ps self annihilation, $0.2-0.5$ ns for free positron annihilation and 1-10 ns for o-Ps annihilating by pick-off. The main limitation of these measurements is the width of the instrumental resolution function, which is typically 250-300 ps. Lifetimes shorter than about one-third of this cannot be measured accurately. Despite the very narrow range of times that can be directly measured, PAL spectroscopy 


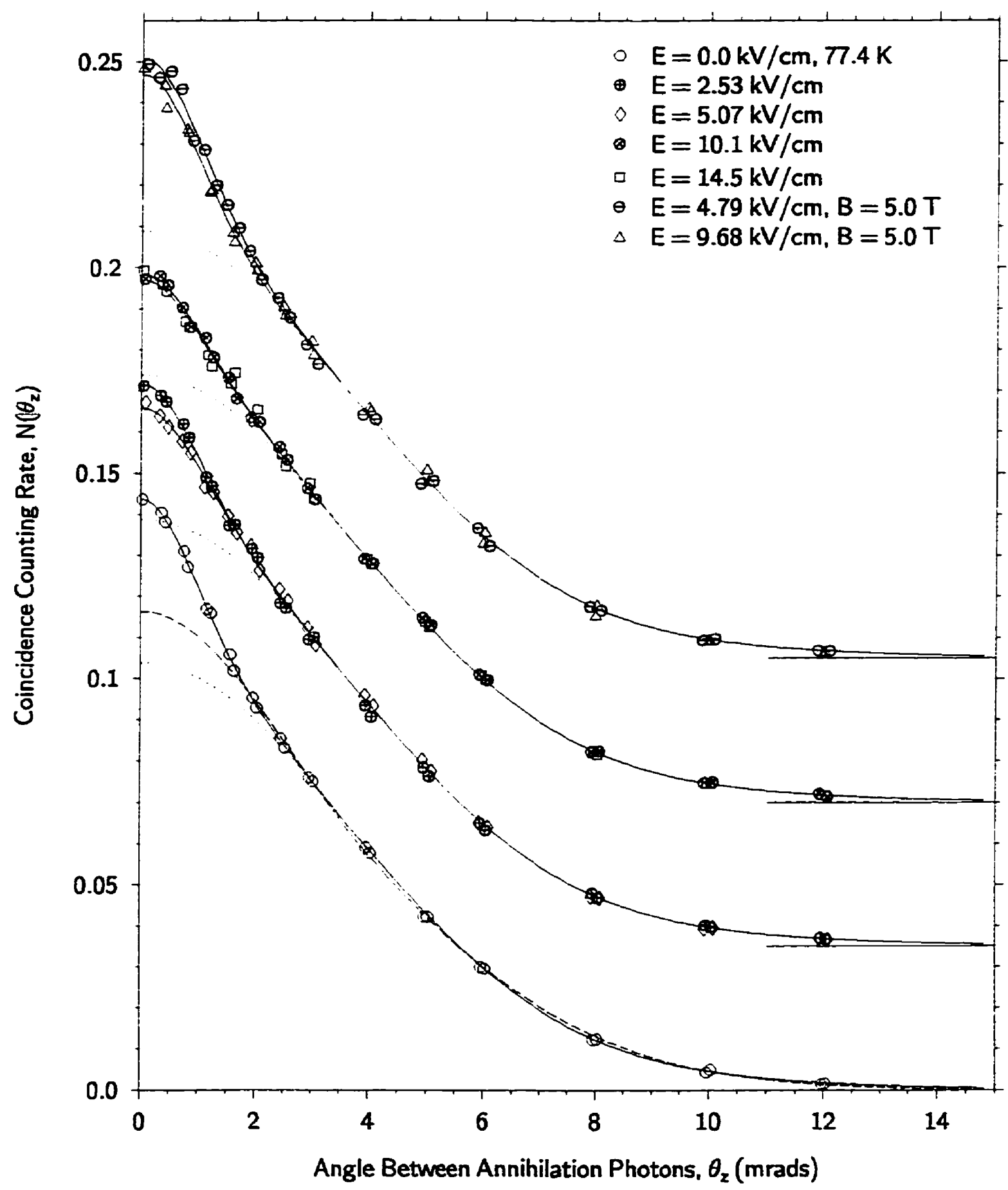

Figure 4.15: Typical ID-ACAR data for positrons annihilating in liquid nitrogen with an applied static electric field. The dotted line corresponds to the best two Gaussian fit to the broad component. The dashed line is an arbitrary Gaussian fit that gives the expected electric and magnetic field dependence of $I_{\mathfrak{n}}$ assuming no Ps spin-conversion processes are present. 


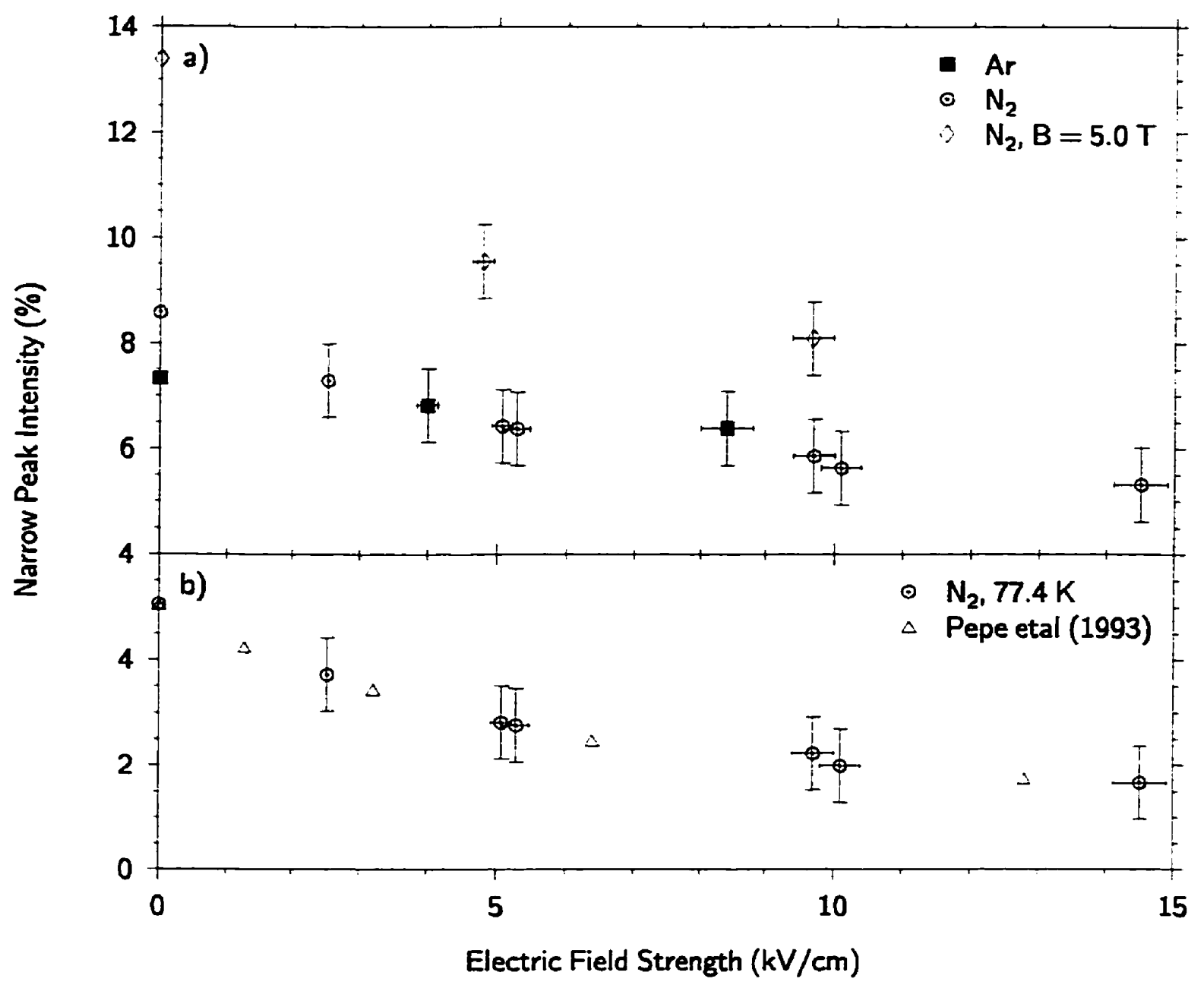

Figure 4.16: The narrow peak intensity as a function of static electric field strength in a) liquid argon and nitrogen using the best fit Gaussians to describe the broad component, and b) in liquid nitrogen using a more peaked two Gaussian fit that reduces all the narrow peak intensities by about $3.5 \%$. The diamonds in a) correspond to measurements in liquid $\mathrm{N}_{2}$ in which both an electric field and a 5.0 T magnetic field were applied. The triangles in b) are the narrow peak intensities implied by the lifetime measurements of Pepe et al. (1993) assuming that the p-Ps:o-Ps ratio is $1: 3$. 


\section{Source}

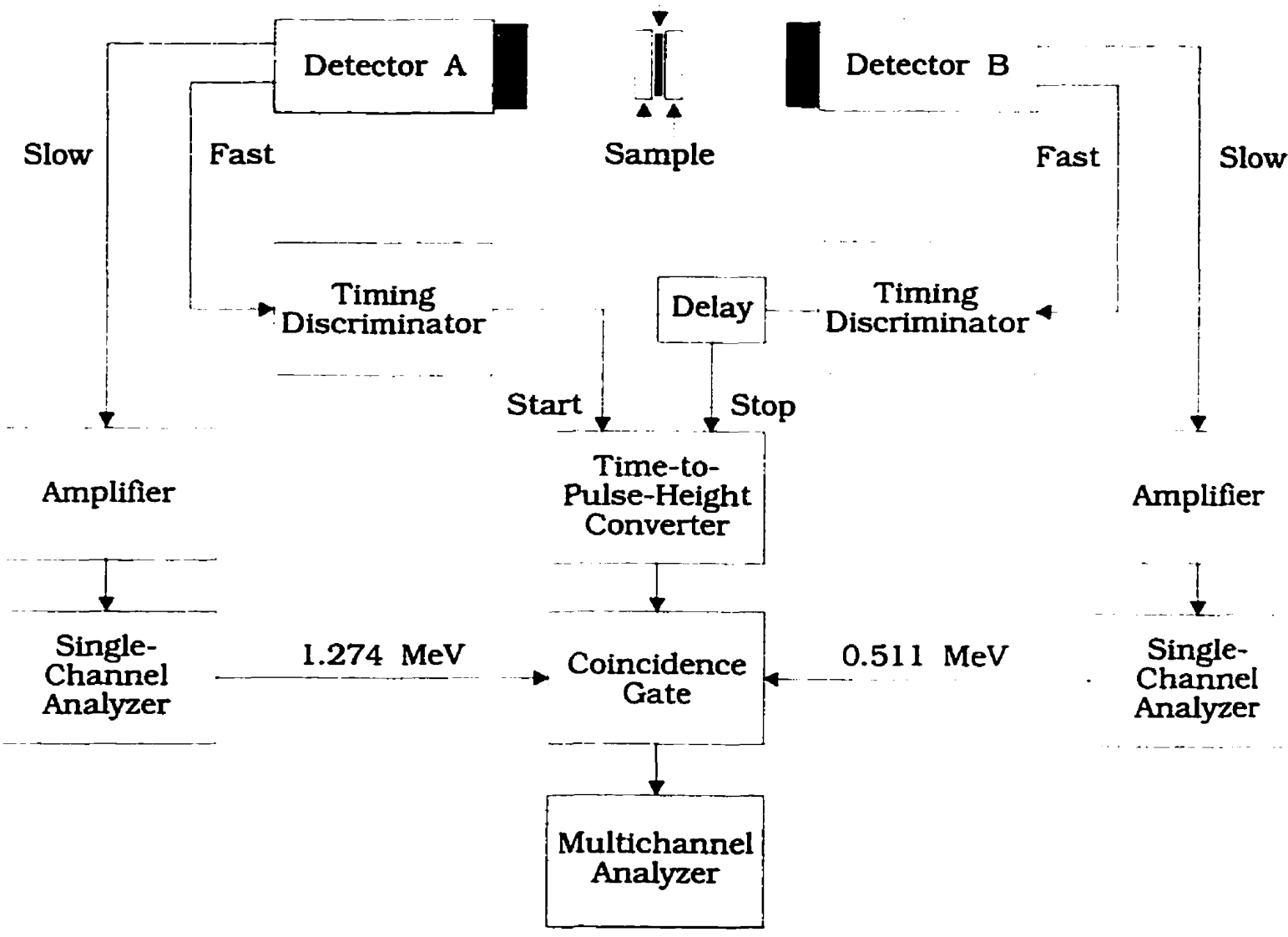

Figure 4.17: A schematic diagram of a conventional fast-slow coincidence positron lifetime spectrometer.

still provides valuable insight on much shorter times because the observed lifetimes and intensities are often significantly affected by earlier events.

The time delay between the birth and annihilation $\gamma$-rays is typically measured using either fast-slow or fast-fast coincidence systems. A conventional fast-slow system is shown in Figure 4.17. The spectrometer consists of two $\gamma$-ray detectors, one for the birth $\gamma$-ray and one for the annihilation $\gamma$-ray, placed on opposite sides of the positron source and sample arrangement. The source is usually prepared by evaporating aqueous a few microcuries $(1-20 \mu \mathrm{Ci})$ of ${ }^{22} \mathrm{NaCl}$ onto a thin metal or plastic foil and then covering it with the same foil. This source is then sandwiched between two identical layers of the sample material, each thick enough to absorb all the positrons. Weak source strengths are used to ensure that only one positron is present in the sample at a time ${ }^{\dagger}$. The detectors consist

$\dagger_{A} 20 \mu \mathrm{Ci}$ source produces $\sim 10^{6}$ positrons per second. Annihilation rates of $\sim 10^{9} \mathrm{~s}^{-1}$ imply that the sample is usually empty positrons and contains one positron $10^{-3}$ of the time, two positrons $10^{-6}$ of the 
of fast plastic or inorganic $\left(\mathrm{BaF}_{2}\right)$ scintillators coupled to fast PMTs. The fast channels measure the time interval between the $\gamma$-rays detected in detectors $A$ and $B$ while the slow channels determine the $\gamma$-ray energies. The energy windows for the single-channel analyzers (SCA) are set so that the slow channel gives a coincidence only when detectors $A$ and $B$ register a $1.28 \mathrm{MeV}$ and a $0.511 \mathrm{MeV} \gamma$-ray, respectively. Signals from the PMT anodes are fed into constant-fraction-timing discriminators which accurately define the arrival time of each $\gamma$-ray. The time to pulse-height converter (TPHC) then produces a pulse whose amplitude is proportional to the time interval between the start signal from detector $\mathrm{A}$ and the stop signal from detector B. The TPHC output pulse is transferred to a multichannel analyzer (MCA) only if there is a coincidence in the slow channel, i.e. only if the start and stop signals have the proper energy values. Fast-fast coincidence systeins use more recent electronics in which the energy analysis is performed in the timing discriminators. This simplifies the system considerably (the slow channel is no longer necessary) and allows for larger counting rates because of practically no pulse pile-up. The instrumental resolution of these spectrometers (250-300 ps FWHM) is limited mainly by the decay of the light centres in the scintillators, the transit time spread in the PMTs and the performance of the timing discriminators.

Typical PAL spectra consist of a million or more counts collected over several hours. Positrons annihilating from a state with a well-defined time-independent annihilation rate $\lambda_{i}$ give rise to an exponential component in the lifetime spectrum of the form $I_{i} \lambda_{i} e^{-\lambda_{i} t} . I_{i}$ measures the relative number of positrons that annihilate at a rate $\lambda_{i}$, while $\lambda_{i}$ gives a measure of the effective electron density at the positron site. Lifetime spectra typically consist of several exponential components, a constant background $B$ due to random coincidences and some scatter $\delta N$

$$
I(t)=\sum_{i=1}^{n} I_{i} \lambda_{i} e^{-\lambda_{i} t}+B+\delta N
$$

where $n$ is the number of distinguishable lifetimes in the data. For a real lifetime spectrum the exponential term in (4.9) should be written as an exponential convoluted with the instrumental resolution function. Real spectra also generally contain a very fast 'prompt' peak due to positron annihilations from inside the positron source material, walls of the sample chamber and/or p-Ps annihilations. Computer fitting procedures (Kirkegaard et al. 1989) are used to remove the source and background contributions and then fit a convoluted sum time, etc. 
Table 4.3: A summary of the known positron lifetime measurements in the condensed media of interest to this thesis. The lifetime spectra for these media consist of at least three components: a $p$-Ps component whose lifetime, $\tau_{1}$, is typically too short to be resolved; a free positron lifetime, $\tau_{2}$; and a longer $o$-Ps lifetime, $\tau_{3}$ which is easily resolved. The area under the $o$-Ps component determines the relative intensity, $I_{3}$.

\begin{tabular}{|c|c|c|c|c|c|}
\hline & $\mathrm{T}(\mathrm{K})$ & $\tau_{2}(\mathrm{~ns})$ & $\tau_{3}(\mathrm{~ns})$ & $I_{3}(\%)$ & Reference \\
\hline${ }^{3} \mathrm{He}(1)$ & 3.05 & & 110 & & [239] \\
\hline \multirow[t]{9}{*}{${ }^{4} \mathrm{He}(1)$} & 4.2 & $1.83 \pm 0.15$ & $91 \pm 5$ & $15.5 \pm 2.5$ & [215] \\
\hline & 4.2 & $2.6 \pm 0.25$ & $120 \pm 20$ & $16 \pm 2$ & [300] \\
\hline & 4.2 & & $112 \pm 6$ & & [73] \\
\hline & $1.5 \rightarrow 4.2$ & $1.67 \rightarrow 1.90 \pm 0.06$ & $88 \pm 8(4.2 \mathrm{~K})$ & & {$[174]$} \\
\hline & $1.7 \rightarrow 4.2$ & & $73 \rightarrow 87 \pm 4$ & $14 \pm 2$ & {$[181]$} \\
\hline & $1.5 \rightarrow 4.2$ & $1.75 \rightarrow 2.01 \pm 0.16$ & $91.4 \rightarrow 99.1 \pm 0.5$ & 15 & {$[121]$} \\
\hline & 4.2 & & $105 \pm 2$ & & [264] \\
\hline & $1.4,4.2$ & & 90,100 & & {$[239]$} \\
\hline & 4.19 & $2.01 \pm 0.002$ & $98.1 \pm 0.2$ & $11.5 \pm 0.7$ & {$[220]$} \\
\hline${ }^{4} \mathrm{He}(\mathrm{s})$ & 1.4 & & $51(26 \mathrm{~atm})$ & & [239] \\
\hline \multirow[t]{3}{*}{$\mathrm{Ne}(\mathrm{s})$} & 20.4 & $0.43 \pm 0.03$ & & & [175] \\
\hline & $5 \rightarrow 22.3$ & $0.336 \rightarrow 0.345 \pm 0.001$ & $4.2 \rightarrow 5.2 \pm 0.1$ & 8 & {$[116]$} \\
\hline & $4.2 \rightarrow 22.0$ & $0.330 \rightarrow 0.340 \pm 0.002$ & $4.43 \rightarrow 4.86 \pm 0.02$ & $7.7 \rightarrow 7.2$ & [117] \\
\hline \multirow[t]{3}{*}{ Ar (l) } & $\begin{array}{c}87.55 \pm 0.15 \\
86\end{array}$ & $0.50+0.02$ & $6.9 \pm 0.7$ & $7 \pm 1$ & $\begin{array}{l}{[216]} \\
{[175]}\end{array}$ \\
\hline & 86.4 & $\begin{array}{c}0.50 \pm 0.02 \\
0.556 \pm 0.005\end{array}$ & $6.50 \pm 0.06$ & $7.0 \pm 0.2$ & [270] \\
\hline & & & 5.8 & 5.5 & {$[277]$} \\
\hline \multirow[t]{4}{*}{ Ar (s) } & 77.3 & $0.43 \pm 0.03$ & & & {$[175]$} \\
\hline & $5 \rightarrow 80$ & $0.391 \rightarrow 0.422 \pm 0.001$ & $2.15 \rightarrow 2.52 \pm 0.05$ & 8.0 & {$[116]$} \\
\hline & $4.2 \rightarrow 80$ & $0.398 \rightarrow 0.441 \pm 0.003$ & $2.19 \rightarrow 2.71 \pm 0.03$ & $7.4 \rightarrow 6.4$ & {$[117]$} \\
\hline & $10 \rightarrow 50$ & $0.22 \rightarrow 0.38$ & $3.25 \rightarrow 2.50$ & $46 \rightarrow 20$ & [246] \\
\hline \multirow{3}{*}{$\begin{array}{l}\mathrm{Xe}(1) \\
\mathrm{Xe}(\mathrm{s})\end{array}$} & 200 & $0.53 \pm 0.04$ & 4.4 & & [294] \\
\hline & 77.3 & $0.40 \pm 0.03$ & & & {$[175]$} \\
\hline & 155 & $0.46 \pm 0.03$ & 2.5 & & {$[294]$} \\
\hline \multirow[t]{2}{*}{$\mathrm{H}_{2}$ (l) } & 20.4 & & $31.2 \pm 1.5$ & $20 \pm 2$ & {$[173]$} \\
\hline & 20.4 & $0.92 \pm 0.04$ & $28.6 \pm 2.3$ & & [174] \\
\hline \multirow[t]{2}{*}{$\mathrm{H}_{2}(\mathrm{~s})$} & & & $16.3 \pm 0.8$ & $20 \pm 2$ & {$[173]$} \\
\hline & 13.0 & $0.80 \pm 0.03$ & $14.6 \pm 1.2$ & & [174] \\
\hline \multirow[t]{4}{*}{$\mathrm{N}_{2}(\mathrm{l})$} & $77.5 \pm 0.1$ & & $11.3 \pm 0.5$ & $15 \pm 2$ & [216] \\
\hline & 77.3 & $0.56 \pm 0.02$ & $10.8 \pm 0.9$ & & [174] \\
\hline & & & 12.2 & 13.9 & [269] \\
\hline & & & 12.5 & $15.2 \pm 0.3$ & [219] \\
\hline $\mathrm{N}_{2}(\mathrm{~s})$ & 62 & $\begin{array}{c}0.48 \pm 0.02 \\
0.51\end{array}$ & $4.9 \pm 0.4$ & & $\begin{array}{l}{[174]} \\
{[238]}\end{array}$ \\
\hline \multirow[t]{2}{*}{$\mathrm{O}_{2}(\mathrm{l})$} & 90.1 & $0.45 \pm 0.02$ & & & {$[174]$} \\
\hline & & $0.495(64 \%)$ & & & {$[277]$} \\
\hline $\mathrm{O}_{2}(\mathrm{~s})$ & 4.2 & $0.38 \pm 0.02$ & & & {$[175]$} \\
\hline
\end{tabular}


of exponentials to extract the relative intensities and lifetimes for an assumed number of components. This analysis contains several sources of uncertainty. Errors in the resolution function and/or the source-background subtraction can significantly affect the magnitude of the intensities and lifetimes, as can the choice for the value of $n$ and the assumptions that go into the analysis. Components with lifetimes within a factor of about two are not resolvable and can result in a curve fitting analysis that can be quite ambiguous.

The results of all known lifetime measurements in the condensed rare and diatomic gases are summarized in Table 4.3. In most of these experiments the exponential fitting is relatively simple since the component corresponding to free positrons annihilating with core electrons is quite distinct from the much longer lifetime resulting from o-Ps pickoff annihilations from inside a localized bubble state. The $p$-Ps are typically too fast to be properly resolved from the other fast annihilations in the prompt peak - the p-Ps component is usually assumed to have a lifetime of $0.12 \mathrm{~ns}$ with an intensity that is onethird of the measured $o$-Ps intensity. Details of these measurements and results are discussed throughout the text as required.

\subsection{Doppler-Broadening Experiments}

In addition to the angular deviations from $180^{\circ}$, the motion of an $\mathrm{e}^{-}-\mathrm{e}^{+}$pair results in a Doppler shift in the energy of the annihilation radiation proportional to $p_{\llcorner}$, as described by (2.39). The subsequent broadening of the $0.511 \mathrm{MeV}$ line shape can be used to obtain information equivalent to that of the ID-ACAR measurements described above. The advantages of these measurements are that they are easy to perform, data acquisition rates are high and only a small source strength ( $\leq 10 \mu \mathrm{Ci}$ ) is required. The positron source-sample arrangement is similar to that used in the lifetime measurements with the annihilation radiation line shape being measured using a high-resolution lithium-drifted or intrinsic Ge crystal. The detection efficiency is an order of magnitude greater than that for 1D-ACAR since the sample can be placed close to the detector and coincidences are not required. Good statistical accuracy can usually be achieved with only a few hours of counting. The major drawback of these lineshape measurements is the relatively poor resolution. The best Ge detectors have an energy resolution of $1.0-1.5 \mathrm{keV}$ at $511 \mathrm{keV}$. This is equivalent to an angular resolution of only 4-6 mrads which is an order of magnitude worse than that of ACAR measurements. This poor resolution makes Doppler broadening 
measurements inappropriate for careful momentum density studies. To date, no Dopplerbroadening experiments of relevance to this work have been performed.

\subsection{Slow Positron Beams}

The experimental techniques described so far all use radioisotopes as their positron source which give an initial positron energy distribution ranging from zero to hundreds of $\mathrm{keV}$. In the more recent slow-positron beam techniques, the positrons are first thermalized at one place and then transported as a monochromatic beam to a sample with which it interacts in a controlled manner. Fast positrons are first obtained either from radioisotopes (typically $\sim 100 \mathrm{mCi}$ of ${ }^{22} \mathrm{Na}$ or ${ }^{58} \mathrm{Co}$ ) or from pair production from bremsstrahlung radiation produced in a LINAC accelerator. The fast positrons are then implanted into a solid (such as $\mathrm{MgO}, \mathrm{Cu}, \mathrm{W}$ ) and thermalized down to energies on the order of $\mathrm{eV}$. These moderators have negative positron work functions so the positrons that reach a surface can escape the bulk back into the vacuum. The thermalized positrons emitted from the solid surface are then focused and transported either magnetically or electrostatically to the sample. Their energies are controlled by potential grids along the beam line. Slow positron beams with energy resolutions of about $0.1 \mathrm{eV}$ and cross sections of $0.1 \mathrm{~cm}^{2}$ have been achieved and used to study a wide variety of phenomena in both atomic and surface physics. Excellent reviews of the techniques and experimental studies can be found in Mills (1983b), Humberston and McDowell (1984), Jean (1988) and Schultz and Lynn (1988).

As previously mentioned in $§ 3.3$, there have been several important slow positron experiments in the rare-gas solids. In one series of experiments, Mills and Gullikson (1986) and Gullikson and Mills $(1986,1989,1990)$ measured the positron and Ps reemission yields from solid $\mathrm{Ne}, \mathrm{Ar}, \mathrm{Kr}, \mathrm{Xe}$ and $\mathrm{N}_{2}$ surfaces as a function of the slow-positron implantation energy. These experiments provided a wealth of useful information. They showed that the Ore gap was effective at producing $P s$ in the rare-gas solids and provided measurements of the positron work function, the Ps binding energy, the Ps formation threshold as well as the exciton and upper Ore gap thresholds. Estimates were also given for the positron mean free path and its average energy loss to phonons below the Ps formation threshold. In a further experiment, Gullikson et al. (1988) measured the energy dependence of the elastic specular reflection of positrons from the (111) surfaces of the rare-gas solids. From the intense first-order 'Darwin top-hat shaped' Bragg peaks, the centre and the width of 
the lowest positron band gap at the $L$ point of the Brillouin-zone boundary were measured (see $§ 5.3 .2)$.

A final set of relevant experiments are those of Tuomisaari et al. (1991). They measured the energy distributions of Ps emitted from the heavier rare-gas solids following positron implantation. Their results again lead to the conclusion that $\mathrm{Ps}$ is formed primarily in the Ore gap in these solids and were able to measure the Ps work function and binding energy. Using a model of energetic Ps diffusion, estimates for the Ps deformation potential (see Appendix C), diffusion constant, energy loss rate and thermalization time were also given. These results indicate that Ps does thermalize rapidly as compared to its lifetime. All of these results are summarized in Table A.5 of Appendix A. 


\section{Chapter 5 \\ The Broad Momentum Component}

The photon momentum distribution arising from positron annihilation in simple condensed gases results in data that is the sum of at least two momentum components. In solid helium and the liquid rare gases, the self-annihilation of $p$-Ps from a self-trapped bubble state results in a narrow momentum component. A narrow peak is also seen in the heavier rare-gas solids, though in this case it is more likely the result of $p$-Ps annihilations from inside various defects and voids. The narrow component is superimposed on top of a much broader momentum component due to positron-core electron annihilations and Ps pick-off annihilations. The focus of this chapter is to study the broad component. The main aim is to obtain a theoretical description for the broad components and determine whether or not the Gaussian fits used in Table 4.2 are reasonable. With this, the broad component can be separated from the narrow one with some confidence thereby allowing a quantitative description of the changes seen in the narrow component.

The chapter starts with a summary of the previous theoretical estimates for the broad component in the rare gases in $§ 5.1$. This includes a description of the positron-gas scattering results, which provide a good first approximation, and the results from previous relevant solid-state calculations. This discussion will show that the existing theoretical calculations do not fit the experimental data very well. If the calculations are reasonable then there is likely one or more 'intermediate' components having a FWHM between that of the broad and narrow peaks. This possibility is discussed in $\S 5.2$. In $§ 5.3$ an attempt is made to improve on the existing broad component calculations for the condensed rare gases. This requires that realistic positron potentials be generated, which is done in $\S 5.3 .1$ and Appendix H. These potentials can then be used to calculate the positron band structure and wave functions, which is done in $\$ 5.3 .2$ and $§ 5.3 .3$, respectively. The positron wave functions are then used in $§ 5.3 .4$ to calculate IPM model estimates for the broad component and positron lifetime. The IPM results are improved upon significantly in $§ 5.3 .5$ where various positroncore electron enhancements are applied. These calculations give theoretical estimates for the broad components that are close to the Gaussian fits used in Table 4.2 and in good agreement with the measured positron lifetimes. 


\subsection{Previous Theoretical Estimates}

\subsubsection{Positron-Gas Scattering Results}

The elastic scattering of low-energy positrons on rare-gas atoms and simple molecules has been the subject of considerable experimental and theoretical interest. The theoretical calculations generally result in a total wave function for the system consisting of a positron scattering from a single atom or molecule. These wave functions may then be used in (2.41), (2.42) and (4.1) to obtain theoretical estimates of the lifetime and momentum distributions for a positron annihilating with an electron from the atom or molecule. The ability of these calculations to describe results for a positron immersed in condensed matter depends on the extent to which the total scattering wave function mimics the total solid-state wave function inside a Wigner-Seitz cell. As will be seen, the scattering results provide a reasonable first approximation to the momentum distribution arising from positron-core electron annihilation.

Much of the theoretical effort has concentrated on positron scattering on helium due to the computational difficulties involved with the heavier rare gases. The first calculation of the electron-positron momentum distribution in helium gas was done by Drachman $(1968,1969)$. He used a trial scattering wave function having a form similar to (2.54)

$$
\Psi_{k}\left(r_{1}, r_{2}, r_{p}\right)=\Psi_{k}\left(r_{p}\right) \Psi_{H e}\left(r_{1}, r_{2}\right)+\phi_{e p}\left(r_{1}, r_{2}, r_{p}\right)
$$

where $\Psi_{H e}\left(r_{1}, r_{2}\right)$ is the ground-state wave function of the target helium atom and $\Psi_{k}\left(r_{p}\right)$ is the scattering function for a positron with momentum $k$. The polarization and distortion of the helium atom by the incoming positron was approximated by the correlation function $\phi_{\text {ep }}\left(\mathbf{r}_{1}, \mathbf{r}_{2}, \mathbf{r}_{p}\right)$ which was written as

$$
\phi_{e p}\left(\mathbf{r}_{1}, \mathbf{r}_{2}, \mathbf{r}_{p}\right)=F\left(\mathbf{r}_{p}\right) G\left(\mathbf{r}_{1}, \mathbf{r}_{2}, \mathbf{r}_{p}\right) \Psi_{\mathrm{He}}\left(\mathbf{r}_{1}, \mathbf{r}_{2}\right)
$$

where $F$ contains the short-range nonadiabatic modifications to the adiabatic correlation function $G$. To make the computations tractable, a simple approximation was used for $\Psi_{\mathrm{He}}\left(\mathbf{r}_{1}, \mathbf{r}_{2}\right)$ which is not readily extendible to the other rare gases. Given this approximation, both $\Psi_{k}\left(\mathbf{r}_{p}\right)$ and $\phi_{e p}\left(\mathbf{r}_{1}, \mathbf{r}_{2}, \mathbf{r}_{p}\right)$ were determined variationally. The resulting total wave function was then inserted into (2.41) and (4.1) to give the momentum distribution shown in Figure 5.1b. The curve has a FWHM of 9.47 mrads, which is somewhat wider than 


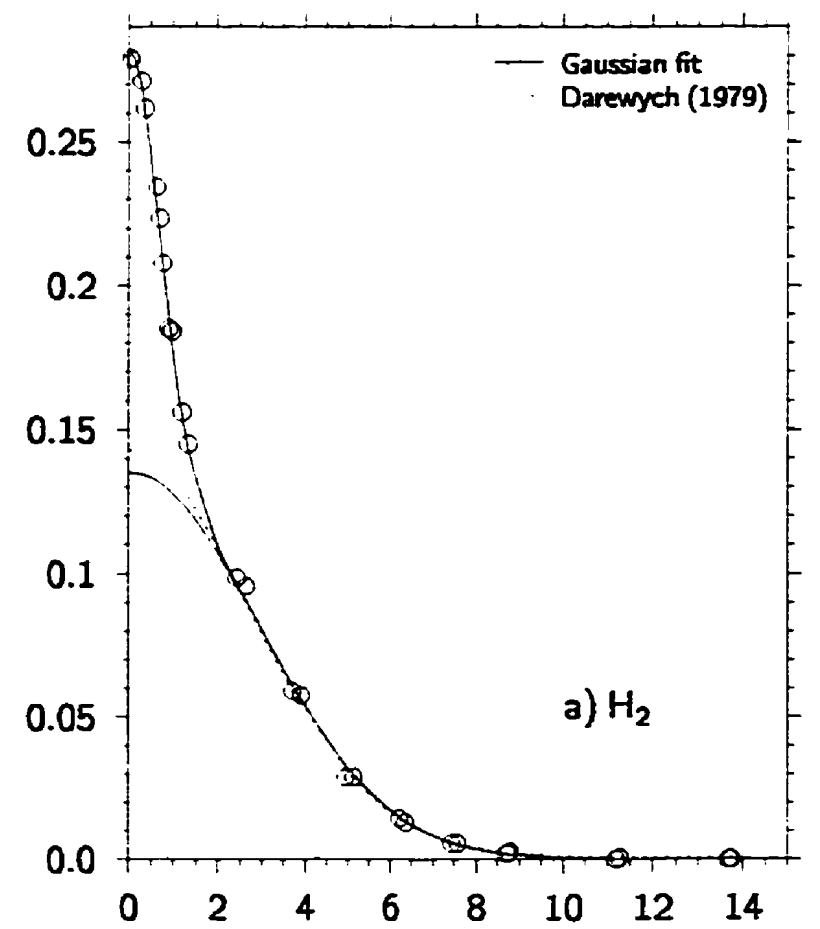

Angle Between Annihilation Photons, $\theta_{2}$ (mrads)

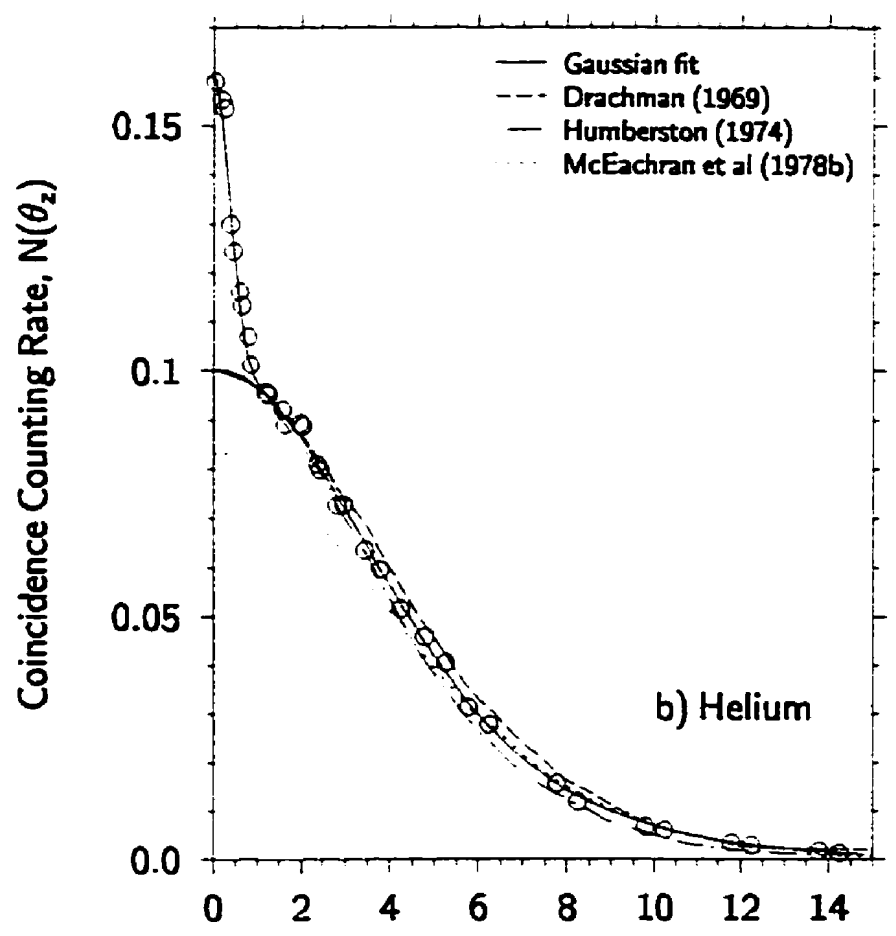

Angle Between Annihilation Photons, $\theta_{\mathbf{z}}$ (mrads)

Figure 5.1: The angular distribution of photons from the positrons annihilating in a) liquid $\mathrm{H}_{2}$ (Briscoe et al.1968) and b) liquid helium. The data has been normalized so that the area under the gaussian fit to the broad component is 1 . The theoretical calculation of Darewych (1979) provides a good fit to the liquid $\mathrm{H}_{2}$ broad component. The broad component in helium is well described by the calculations of Drachman (1969) and Humberston (1974). The McEachran et al.(1978b) calculation predicts a broad component in helium that is significantly wider than is suggested by experiment. 
the experimental estimate of $8.8 \pm 0.2$ mrads and results in a underestimate of the broad component at low angles.

Humberston $(1973,1974)$ formulated a more realistic model for the low-energy positronhelium scattering problem. Using variational methods he calculated a total wave function similar to (5.1) except the helium ground-state wave function used by Drachman was replaced by a more realistic five-term Hylleraas-type function. Humberston's results, as extended by Campeanu and Humberston (1975), for the positron-helium scattering length, the low-energy s-wave phase shifts, the elastic scattering cross-section and the annihilation rate parameter $Z_{\text {eft }}$ were significantly different from those of Drachman and were in better agreement with the experimental data. The calculated momentum distribution, shown as the dash-dot line in Figure $5.1 \mathrm{~b}$, has a FWHM of 8.6 mrads and gives somewhat better agreement with experiment. Humberston also performed the same calculations using Drachman's simpler helium wave function. These results were similar to those of Drachman (1968) - the calculated broad component had a FWHM of 9.6 mrads.

McEachran et al.(1977) have formulated the elastic scattering of positrons from raregas atoms in terms of a nonvariational polarized orbital approximation. In this case, the total wave function is again similar to (5.1) and (5.2) except that now there is the further restriction that $F\left(r_{p}\right)=\Psi_{k}\left(r_{p}\right)$. Furthermore, both the unperturbed ground-state target wave function $\Psi\left(r_{1}, r_{2}, \ldots\right)$ and the correlation term $G\left(r_{1}, r_{2}, \ldots, r_{p}\right) \Psi\left(r_{1}, r_{2}, \ldots\right)$ are written as determinants to ensure proper antisymmetrization. $\Psi_{\mathbf{k}}\left(r_{p}\right)$ is determined by projecting the full Schrödinger equation onto the ground state of the atom - i.e. the equation $\left\langle\Psi\left(\mathbf{r}_{1}, \mathbf{r}_{2}, \ldots\right)|H-E| \Psi_{k}\left(\mathbf{r}_{1}, \mathbf{r}_{2}, \ldots, \mathbf{r}_{p}\right)\right\rangle$ is solved, where $H$ is the Hamiltonian for the scattering problem. The main virtue of these calculations is that while they are sophisticated enough to give a reasonable description of the scattering problem, they are also computationally simple enough to be extended to the heavier rare gases. The electron-positron momentum distributions calculated by McEachran et al. $(1977,1978 \mathrm{a}, 1978 \mathrm{~b}, 1979,1980)$ are shown by the dotted lines in Figures 5.1b and 5.2. These curves have FWHM of about $9.6,14.8,10.9,9.8$ and 8.7 mrads in helium through to xenon, respectively. The best fit Gaussians to the Varlashkin (1971) results give a broad component FWHM of $9.0 \pm 0.2$ and $8.2 \pm 0.2$ mrads for krypton and xenon, respectively. In each case, the calculated momentum distribution appears to be wider than is suggested by the experimental data and as a result the theory tends to underestimate the fitted broad component at low angles and overestimate it at large angles. 

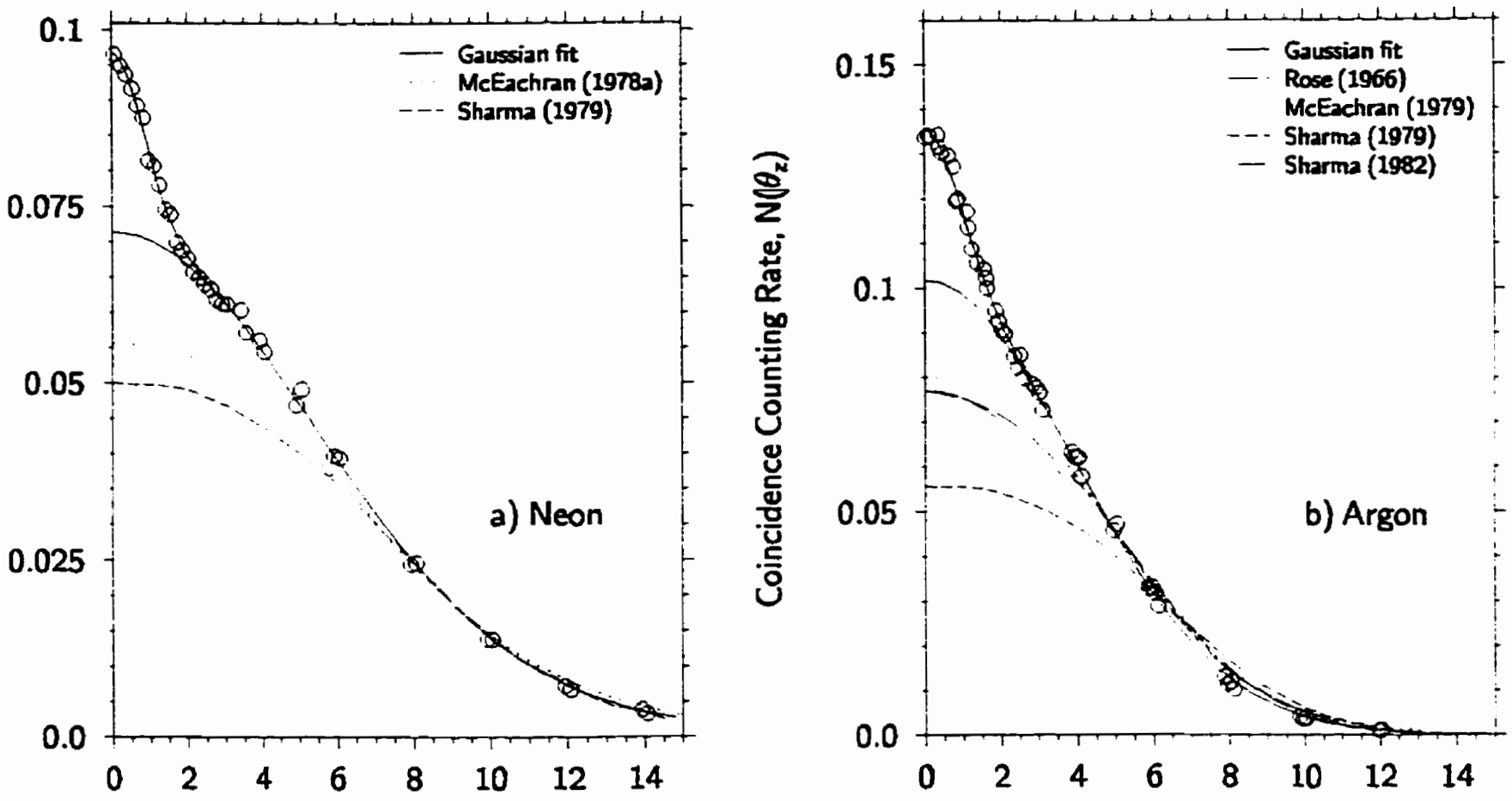

Angle Between Annihilation Photons, $\theta_{2}$ (mrads)

Angle Between Annihilation Photons, $\theta_{z}$ (mrads)
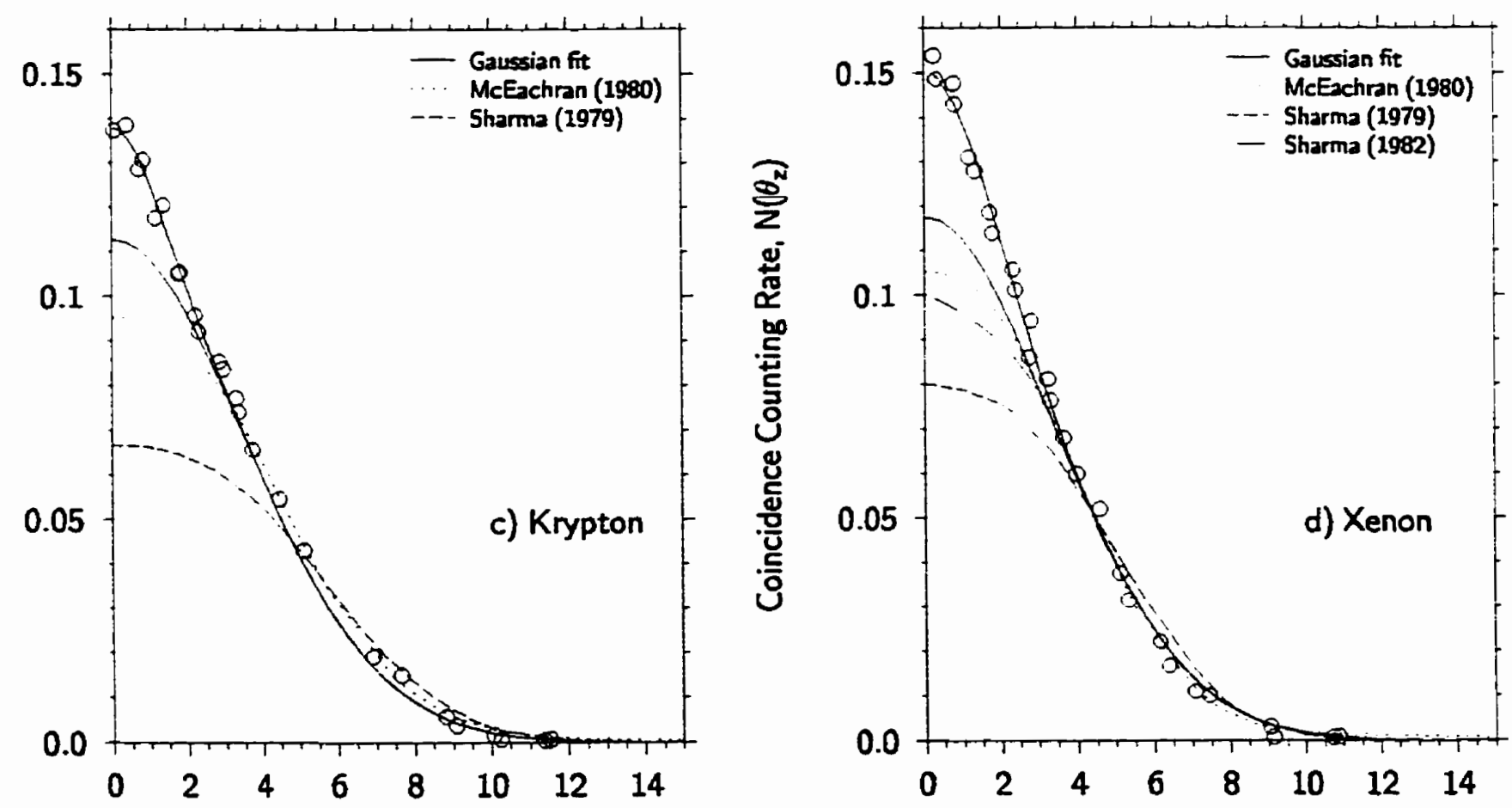

Angle Between Annihilation Photons, $\theta_{z}$ (mrads)

Angle Between Annihilation Photons, $\theta_{2}$ (mrads)

Figure 5.2: The angular distribution of photons from the positrons annihilating in liquid a) neon, b) argon, c) krypton and d) xenon. The data for krypton and xenon has been taken from Varlashkin (1971). The data has been normalized so that the area under the Gaussian fit to the broad component is 1. For each liquid the existing theoretical calculations for the broad component have also been plotted. In general these estimates are significantly wider than what is suggested by experiment and do not provide much help in separating the broad and narrow components. 
Figure 5.1a compares the experimental data in liquid $\mathrm{H}_{2}$ from Briscoe et al. (1968) to the theoretical calculation of Darewych (1979). The theoretical curve was normalized to give the best fit to the data at larger angles. Darewych described the positron- $\mathrm{H}_{2}$ system by using the wave function (5.1) without the correlation term and by assuming a simple one-centre expansion for the $\mathrm{H}_{2}$ wave function. This calculation gives a theoretical estimate that has a FWHM of about $6.6 \mathrm{mrads}$ and appears to fit the data quite nicely. However, it remains uncertain how accurate this relatively simple estimate actually is. To date, no similar calculations for positrons scattering from either $\mathrm{N}_{2}$ or $\mathrm{O}_{2}$ molecules exist.

\subsubsection{Solid-State Calculations}

Despite a large body of positron research in condensed matter, there have been few studies specifically concerned with positrons annihilating with core electrons. The few that have been attempted and applied to the condensed rare gases were mentioned in $\$ 2.5 .4$. However, there still remains a lack of information and understanding about positron-core electron annihilation and the associated positron-electron enhancement factors and their momentum dependence.

One of the first attempts to calculate the momentum distribution arising from positron core-electron annihilations was that of Rose and DeBenedetti (1965). They extended the Wigner-Seitz calculation, first illustrated by Berko and Plaskett (1958), to calculate the 1D-ACAR in solid argon. Their results, as corrected by Rose and Woll (1966), are very similar to those of McEachran et al. (1979), as shown by the large dashed line in Figure 5.2. The calculated curve has a FWHM of 11.2 mrads which is significantly larger than the Gaussian fit FWHM of 9.2 mrads. When fit to the larger angles the Rose and DeBenedetti curve falls well short of the Gaussian fit at smaller angles. The calculation gives a positron lifetime of $1.25 \mathrm{~ns}$. This is significantly longer than the measured value of $0.43 \pm 0.03 \mathrm{~ns}$ (Lui and Roberts 1962, 1963a, 1963b) in solid argon and $0.556 \pm 0.005 \mathrm{n}$ s (Spektor et al. 1971) in liquid argon. Woll and Rose (1966) used a more sophisticated approach in which the positron-core electron correlations were included directly in the twoparticle positron-electron wave function. This calculation gave an improved lifetime result of 0.53 ns. However, the calculated $1 D$-ACAR was significantly wider (FWHM $=13.3 \mathrm{~m}$ rads) and in very poor agreement with experiment, making this approach of little practical use. 
Salvadori and Carbotte (1969) used a method first developed by Kahana (1960, 1963) for metals, and then extended by Carbotte $(1966,1967)$, to calculate the momentum distribution and lifetime in solid argon. Their original calculations gave a good fit to the IDACAR as well as a reasonable lifetime result. However, Sharma and Majumdar (1979) and Bonderup et al. (1979) discovered errors in this work which, when corrected, gave a similar positron lifetime $(0.52 \mathrm{~ns})$ but a much poorer momentum distribution. The dashed lines in Figure 5.2 show the Sharma and Majumdar (1979) for the heavier rare-gas solids. The FWHM of the calculated curves are 15.9, 12.6, 11.6, and 9.9 mrads for solid neon, argon, krypton, and xenon, respectively. For each rare gas, the broad component calculated using the Salvadori and Carbotte theory is virtually identical to Sharma and Majumdar (1979) simple independent particle model calculation shown in Figure 5.8. Despite the rather poor fit to the momentum distribution, the theory does predict positron lifetimes of $0.53 \mathrm{~ns}$ for solid neon and krypton and 0.57 ns for xenon. This compares reasonably well to measured values of $0.43 \pm 0.03 \mathrm{~ns}$ for neon and $0.40 \pm 0.03 \mathrm{~ns}$ for solid xenon (Lui and Roberts 1963b). By adding several simplifying assumptions to the Salvadori and Carbotte (1969) theory, Chiba et al. (1977) obtained a simple result describing the momentum dependent positronbound electron correlation enhancement. Using this theory, Sharma and Singh (1982) recalculated the momentum distribution in solid argon and xenon. Their results, shown by the dash-dot lines in Figure 5.2, gave a somewhat narrower angular correlation curves the FWHM was 11.2 and 8.8 mrads in solid argon and xenon, respectively.

In helium and $\mathrm{H}_{2}$, the FWHM of the narrow peaks is sufficiently different from their broad component that the peak separation is relatively easy. The theoretical calculations of the helium and $\mathrm{H}_{2}$ broad components are also of high enough quality to be useful in the separation of the components. However, this is not the case for the heavier rare gases where the wider narrow peaks make the separation from the broad component far more difficult. If it is assumed that the estimates of the broad and narrow components obtained from the simple-minded Gaussian fits are reasonable, then it appears that all the theoretical estimates of the broad component are consistently too wide. These estimates should provide reasonable fits to the data at large angles (momenta). The reason is that for large momenta, the momentum dependent enhancement factors due to positron-electron correlation should approach a constant value of about 1 to 1.5 (Bonderup et al. 1979). When the theoretical calculations are scaled to fit the experimental results at higher momenta, away from the narrow peak, they fall well short of the Gaussian fit to the broad component at low momenta. 
Typically the difference between the two estimates is a component with a FWHM of about 4 mrads and an intensity of less than $10 \%$. If the theoretical calculations are thought to be reasonable, then one must consider the possible existence of one or more 'intermediate' components having a FWHM between that of the broad and narrow peaks. However, it may also be that the current theory describing the broad component is simply not yet adequate. More specifically, the momentum-dependent enhancement factors calculated at low momenta may not be correct. Both of these possibilities are discussed below.

\subsection{Possibility of Intermediate Momentum Components}

Even in relatively simple condensed media there exists a number of possible interactions and/or chemical reactions that could result in an intermediate momentum component in the angular correlation curves. The first possibility are Ps pick-off annihilations. The assumption that pick-off annihilations fall under the broad component is based on the idea that the momentum distribution from either a free positron or Ps undergoing pick-off annihilation from the bubble state should be approximately the same. This assumption can be checked by changing the relative fraction of Ps pick-off annihilations, either by applying electric or magnetic fields, by adding $\mathrm{O}_{2}$ impurity, or by undergoing a phase change between liquid and solid, and then looking for changes in the 1D-ACAR spectra. In all these cases, the momentum component under the narrow peak, as defined by the Gaussian fits in $§ 4.3 .1$, remained unchanged to within experimental uncertainty. This implies that the shape of the Ps pick-off momentum component is essentially the same as the positron component. The fact that the Gaussian fit to the 'broad' component is virtually identical in both liquid and solid also shows that positron trapping in defects in solids cannot be used to explain a middle component.

The possibility of positron and/or Ps pick-off annihilations with an unattached electron from the positron spur might also give rise to an intermediate momentum component. In helium and liquid neon, excess electrons are known to self-trap into bubble states very quickly, thereby preventing their interaction with Ps trapped in a bubble. It is possible for free positrons to interact with the electron bubble to either form Ps or to simply annihilate with some intermediate momentum distribution. This, however, is unlikely. To understand why, it is useful to compare helium and neon. Helium has a low polarizability which implies that a positron could easily find an electron bubble. However, the Gaussian fits and theo- 
retical estimates of the broad component imply that no intermediate component is present. On the other hand, liquid neon, which has a possible intermediate component, has a higher polarizability making it less likely that a positron could find an electron bubble. Furthermore, solid neon, which has a similarly shaped 'broad' component to liquid neon, has a high excess electron mobility (i.e. the excess electrons are not trapped in bubbles). This implies that the positron finding an electron bubble mechanism could not be used to account for the middle component in solid neon or in the other heavier condensed rare gases which also have a high electron mobility. Similarly, the possibility of free positrons annihilating with an excess free electron giving rise to an intermediate component cannot account for the intermediate component in liquid neon. Finally, the relatively low spur electron density makes it unlikely that the above processes could account for the observed intensities of the apparent intermediate components.

Another possible interaction with excess spur electrons is the formation of positronium ion $\left(\mathrm{Ps}^{-}\right)$-like states. It is most likely that $\mathrm{Ps}^{-}$would exist in a bubble state in liquids or trapped at a defect in solids and that it would be formed by an electron being captured by a Ps that is already localized. Even in the heavier rare gases with their high electron affinity, it is likely that $\mathrm{Ps}^{-}$bubble is more stable energetically than a Ps bubble. Furthermore, the higher zero-point momentum of the $\mathrm{Ps}^{-}$bubble would result in an intermediate momentum component. However, the formation of electron bubbles make it unlikely that $\mathrm{Ps}^{-}$bubbles could form in liquid neon and therefore explain its apparent intermediate component. It is also unclear if $\mathrm{Ps}^{-}$could form on time scales and in sufficient quantities to be observed experimentally. To date, no lifetime or ACAR studies have attributed a component to annihilations from a $\mathrm{Ps}^{-}$state.

A final possibility that is considered here is the formation of various positron and Ps bound states. Helium is definitely unable to bind a positron (Golden and Epstein 1974; Schrader 1979; Karl et al.1984). No experimental evidence exists to support a positron-Ne bound state and there is good theoretical evidence against it (Golden and Epstein 1974). The other rare gases have negative positron affinities, implying no bound state, however the affinities are small enough that bound states cannot be definitely ruled out (Schrader 1979). Karl et al.(1984) used a simple model to calculate possible Ps-atom bound states and were unable to find any for the rare gas atoms. However, even if bound positron or Ps states did form it is unlikely that they would result in a momentum distribution significantly different from the free positron or Ps pick-off annihilations since in all cases the positrons 
are annihilating with core electrons. Interactions between positive ions in the spur and Ps would be unlikely. If they did occur they would likely result in the oxidation of the Ps and would therefore contribute to the broad momentum component.

\subsection{Calculation of the Broad Momentum Component}

From the discussion in $\$ 5.2$ it appears that there is no single mechanism that would convincingly account for a middle component in each of the heavier condensed rare gases. This implies that either the Gaussian fits and/or the current theoretical estimates are poor descriptions of the actual shape of the broad component. While it is difficult to know how well the Gaussian fits actually represent the various momentum components, there are several good reasons to expect that they are reasonable first approximations. For each rare gas, the same Gaussian fit provides a good fit to the large angle data (i.e. $\theta>4$ mrads) in both the liquid and solid state, in various electric and magnetic fields and $\mathrm{O}_{2}$ concentrations. In helium, the broad component is relatively well defined and is described quite nicely by the Gaussian fit.

If the Gaussian fits are reasonable, then the current theoretical calculations for positrons annihilating with core electrons are not yet satisfactory. The aim of this section is to use the solid-state techniques described in $\$ 2.4 .1$ and $\$ 2.5 .2$ to first calculate the IPM positron momentum distribution and lifetime in the condensed rare gases. An attempt will then be made to use the core enhancements described in $\$ 2.5 .4$ to obtain more reasonable estimates for the shape of the broad component and the positron lifetime.

\subsubsection{Positron Potentials}

To calculate estimates for the broad momentum component and the positron lifetime it is necessary to first calculate the electron-positron momentum density. This, in turn, requires estimates of the electron and positron wave functions and some description of the correlations between them. The solid-state electron wave functions are described using the tight binding approximation (see §2.5.3). In this approximation, the Bloch core-electron wave functions are written in terms of the corresponding atomic orbitals, for which relatively simple analytic forms already exist, localized about each lattice site. More sophisticated 
self-consistent band structure calculations could have been used to calculate the solid-state electron wave functions. However, it is unlikely that this complication would produce significantly improved results given the crudeness of other parts of these calculations. The positron solid-state wave functions will be calculated using the techniques of $\$ 2.4 .1$. These calculations require a reasonable approximation of the crystal potential seen by a positron in the condensed material. This section summarizes the calculation of the positron potentials for the rare gases. The details are left to Appendix $\mathrm{H}$.

The total potential seen by a positron may be written as

$$
V(\mathbf{r})=\sum_{\nu} v\left(\mathbf{r}-\mathbf{R}_{\nu}\right)
$$

where the sum goes over the atomic positions in the lattice $\mathbf{R}_{\nu}$. The potential arising from a single atom is

$$
v(\mathbf{r})=-v_{\text {Coul }}(\mathbf{r})+v_{\text {corr }}(\mathbf{r})
$$

where $v_{\text {Coul }}(\mathbf{r})$ is the Hartree part of the electron crystal potential due to the nuclei and the crystalline charge density $n(\mathbf{r})$. The calculation of $v_{\text {Coul }}$ is described in detail in Appendix H. $v_{\text {corr }}$ is the electron-positron correlation potential describing the energy lowering due to the short-range pileup of electrons around the positron. It tries to account for the distortion of the positron wave function from its IPM value resulting from the electron pileup. This pileup also enhances the positron annihilation rate, increases the positron's effective mass and reduces the positron interaction beyond the screening cloud. The response of the electrons to the positron however depends on their 'character' - it is largest for nearly free conduction electrons and is smallest for localized core electrons. In metals, $v_{\text {corr }}$ is generally calculated using the local density approximation (LDA) and is determined from many-body calculations for a free positron in a homogenous electron gas (Arponen and Pajanne 1979a, $1979 \mathrm{~b})$. In semiconductors, the positron-electron gas correlation requires a reduction factor to account for the imperfect screening of the positron (Puska et al. 1989). Unfortunately, these many-body calculations for the correlation potential break down in the condensed rare gases since the screening cloud around the positron is not isotropic.

In insulators, such as the rare-gas solids, the pileup of electrons around the positron is a result of the polarization of the atomic orbitals. The long-range behaviour of the attractive polarization interaction is proportional to $-1 / r^{4}$. At small distances the correlation potential remains finite but its actual form is unknown. Several different models were tried 
to describe the polarization effects in the rare-gas solids. The first one was successfully used by Schrader (1979) in positron-rare-gas atom scattering calculations. In this simple semiempirical model the positron-atom correlation potential had the required long-range behaviour beyond a fitted cutoff parameter $r_{0}$

$$
V_{\text {corr }, S}(r)=-\frac{e^{2}}{4 \pi \epsilon_{o}} \frac{\alpha}{2 r^{4}}
$$

for $r \geq r_{o}$ and

$$
V_{\mathrm{corr}, \mathrm{S}}(r)=-\frac{e^{2}}{4 \pi \epsilon_{o}} \frac{\alpha}{2 r_{o}^{4}}
$$

for $r<r_{0} . \alpha$ is the atomic polarizability of the atom. Below $r_{0}$ the potential is simply equal to the value at $r_{o}$, giving rise to a discontinuous derivative at $r_{o}$. The results of positron band structure calculations below in $\$ 5.3 .2$ suggest that this model overestimates the correlation potential. To overcome this, a second smooth correlation potential, due to Abarenkov and Antonova (1974) was also tried

$$
v_{\text {corr }, \mathrm{AA}}(r)=-\frac{e^{2}}{4 \pi \epsilon_{o}} \frac{\alpha}{2\left(r^{2}+r_{1}^{2}\right)^{2}}
$$

This model also contains a parameter, $r_{1}$, that was arbitrarily varied to obtain potentials that gave the best fit to experimental results. The larger the $r_{\mathfrak{l}}$ parameter, the more the total potential becomes like the bare Coulomb potential. Puska and Nieminen (1992) have already used this model with some success in their band structure calculations for the rare-gas solids.

For the APW calculations, the required muffin-tin potentials were constructed using a technique first suggested by Mattheiss (1964). The potential from a single atom, $v(r)$, was calculated first. The heavier rare-gas solids are all face-centered cubic. The sphericallysymmetric contributions from neighbouring atoms in the lattice were then included by using the Löwdin alpha-function expansion, described in Appendix G. This resulted in a total potential $V(\mathbf{r})$ that was spherically symmetric inside the muffin-tin spheres inscribed inside each Wigner-Seitz cell. Outside the muffin-tin spheres, $V(\mathbf{r})$ was simply set to its average value. These potentials were also used for the Wigner-Seitz calculations. The pseudopotential calculations do not require the total potential $V(\mathbf{r})$. Instead the single atom potential $v(r)$ is used since the summation in (5.3) is built into the calculation.

The total positron potentials, $V(r)$, for each of the model correlation potentials are shown in Figure 5.3. The parameters used to calculate these potentials are summarized in 

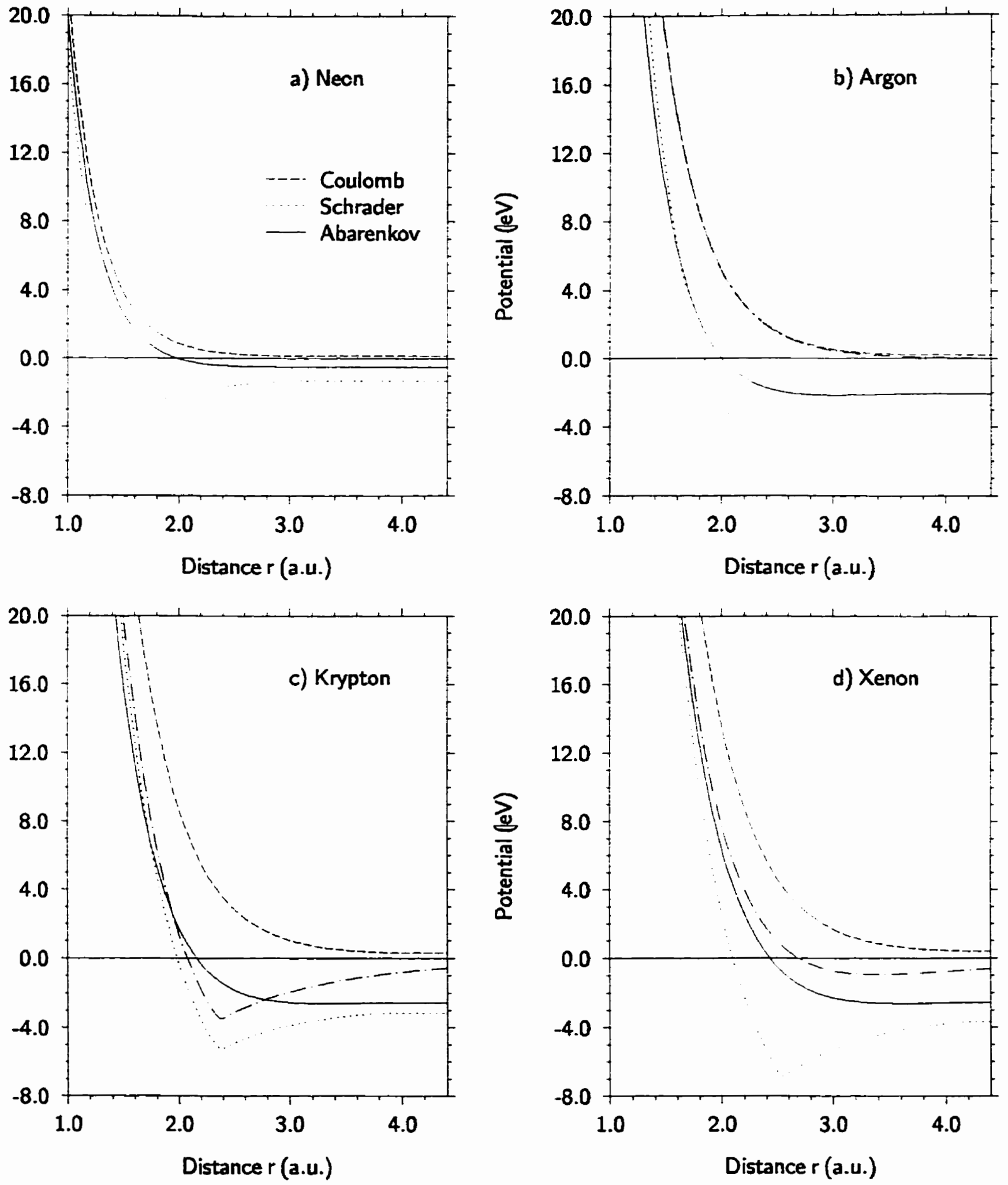

Figure 5.3: The total positron potential, $V(r)$, around an atom in the rare-gas solids using different models for the positron-core electron correlation potential. These potentials were used in the APW and Wigner-Seitz calculations. The dashed line shows the bare Coulomb potential seen by the positron. The dotted line includes the positron correlation potential first suggested by Schrader (1979). The solid line corresponds to the positron potential that includes the correlation potential of Abarenkov and Antonova (1974). Each of these potentials includes the contributions from the surrounding atoms. The dash-dot line in Figures $\mathrm{b}, \mathrm{c}$, and $\mathrm{d}$ show the Coulomb, Schrader, and Abarenkov potentials, $v(r)$, respectively, around a single atom. 
Table H.1. The bare Coulomb potentials, shown by the dashed lines, provide an upper limit on the positron potential. The Schrader potentials, given by the dotted lines, were calculated using the parameter values from Schrader (1979). The results from band-structure calculations below indicate that these potentials provide a lower limit for the positron potentials. The solid lines in Figure 5.3 include the correlation potential suggested by (5.6). Puska and Nieminen (1992) used these potentials with a parameter value of $r_{1}=1.70$ a.u. for all the rare-gas solids in their positron band structure calculations. They found that the first positron band gap at the $L$ point, $E_{g, L}$, was relatively insensitive to the value of $r_{1}$. Their calculated values of $E_{g, L}$ were in excellent agreement with experiment. In the present calculations the parameter $r_{1}$ was chosen to be 2.00, 1.50, 1.60, and 2.05 a.u. for $\mathrm{Ne}, \mathrm{Ar}$, $\mathrm{Kr}$, and $\mathrm{Xe}$, respectively. These values gave potentials that fell in between the Coulomb and Schrader potentials and resulted in positron band structures that best fit the available experimental data. The plots for $\mathrm{Ar}, \mathrm{Kr}$, and $\mathrm{Xe}$ also include a dash-dot line showing the Coulomb, Schrader, and Abarenkov potentials around a single atom, respectively. These are samples of the $v(r)$ potentials used in the pseudopotential calculations. The difference between the $V(r)$ and $v(r)$ clearly demonstrates the that the surrounding atoms add a significant contribution to the correlation potential about any given atom in the solid.

\subsubsection{Positron Energy Levels}

Using the positron potentials it is possible to calculate both the positron band structure and solid-state wave functions. The band structure was calculated using the APW method described in $\$ 2.4 .1 .2$. The quality of these calculations was checked by first calculating the electron band structure for solid argon using an electron potential described in Appendix H. The agreement with previous calculations (Mattheiss 1964) was excellent.

Figure 5.4 shows the positron band structure for the rare-gas solids. These were calculated using the Abarenkov potentials shown in Figure 5.3. The positron energies are measured with respect to the crystal zero level, which is defined as the Coulomb potential far away from a single atomic sphere. This choice gives a crystal zero that is common for both electrons and positrons. Thermalized positrons are at the bottom of the lowest energy band at $k=0$. The positron chemical potential $\mu_{+}$is defined as the difference between the bottom of the lowest band and the crystal zero. For neon $\mu_{+}$is positive, while for the other rare-gases it is negative. The electron chemical potential, $\mu_{-}$, is similarly defined 

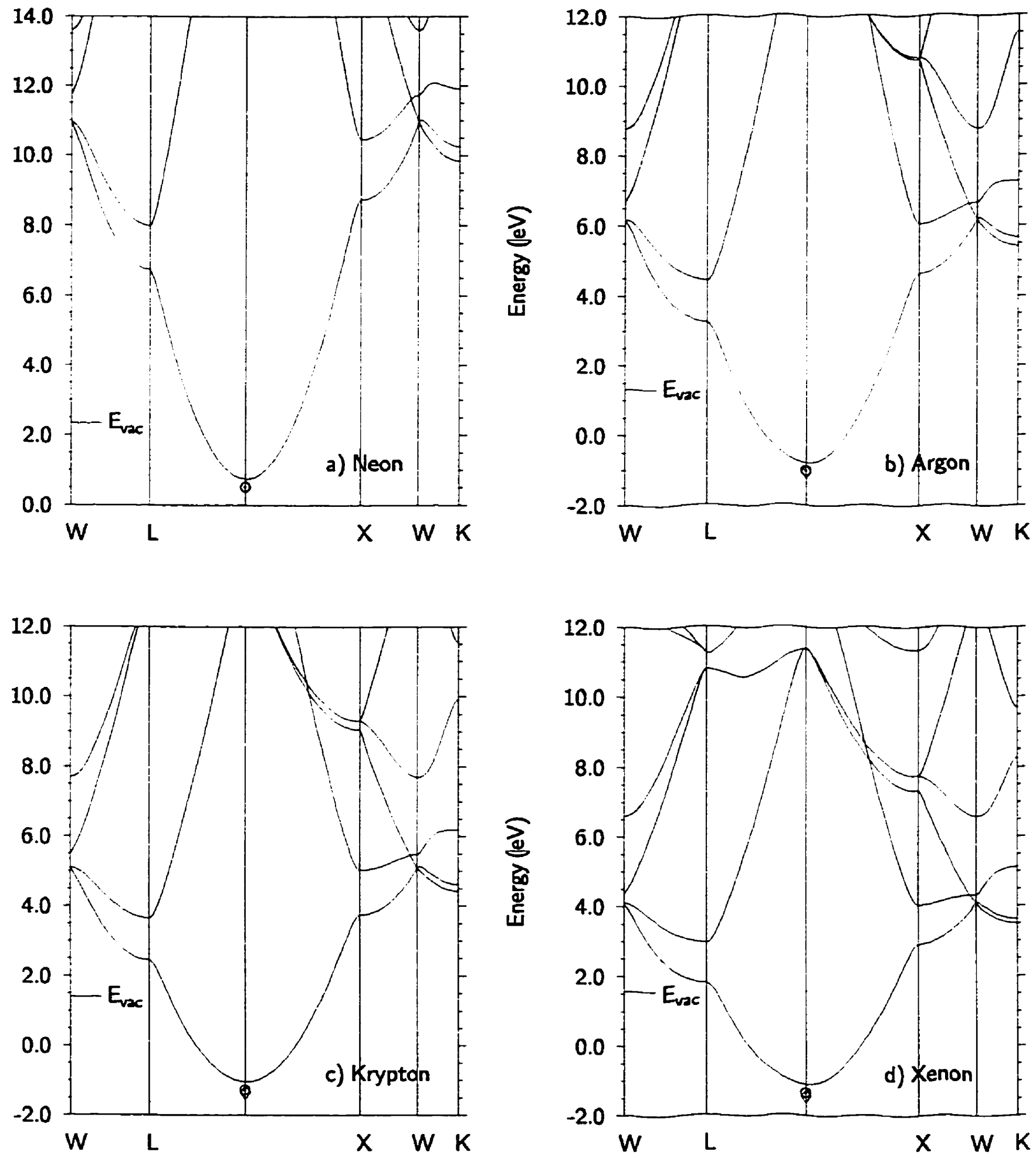

Figure 5.4: The positron band structure for the rare-gas solids. The energies were calculated using the APW method and are plotted along lines in the first Brillouin zone joining the points $\Gamma(k=0), L$, and $W$ and $\Gamma, X, W$, and $K$. The circles and diamonds are the calculated Wigner-Seitz and $k=0$ pseudopotential energies, respectively. The crystal zero used here was defined by the energy zero in Figure 5.3. $E_{\mathrm{vac}}$ denotes the vacuum energy level. 
Table 5.1: Results of the positron band gap calculations in the rare-gas solids. $\mu_{+}$ is the calculated positron energy at the bottom of the band. $E_{g, L}$ is the width of the first band gap at the $L$ point shown in Figure 5.4 while $E_{\text {Bragg }}$ is the energy at the center of these band gaps. Results are given for the band structures derived using the Coulomb, Schrader and Abarenkov potentials described above. The Abarenkov results correspond to those shown in Figure 5.4. The experimental results (expt) obtained by Gullikson et al. (1988) are also given for comparison. These experimental results were measured with respect to the vacuum energy level. The difference between the crystal zero used in these calculations and the vacuum level is the dipole potential step $\Delta$. The value of $\Delta$ given here was taken from the electron band structure calculations of Puska and Nieminen (1992). All energies are in $\mathrm{eV}$.

\begin{tabular}{lcccc}
\hline \hline & $\mathrm{Ne}$ & $\mathrm{Ar}$ & $\mathrm{Kr}$ & $\mathrm{Xe}$ \\
\hline Couloub potential & & & & \\
$\mu_{+}$ & 1.89 & 2.39 & 2.66 & 2.74 \\
$E_{\text {Bragg }}$ & 8.51 & 7.02 & 6.77 & 6.23 \\
$E_{g, L}$ & 1.32 & 1.48 & 1.46 & 1.33 \\
$\phi_{+}$ & 0.45 & -1.08 & -1.26 & -1.77 \\
Schrader potential & & & & \\
$\mu_{+}$ & -0.22 & -1.76 & -2.18 & -2.93 \\
$E_{\text {Bragg }}$ & 6.38 & 2.83 & 1.88 & 0.50 \\
$E_{g, L}$ & 1.09 & 1.05 & 1.09 & 0.92 \\
$\phi_{+}$ & 2.56 & 3.18 & 3.58 & 4.50 \\
Abarenkov potential & & & & \\
$\mu_{+}$ & 0.74 & -0.80 & -1.04 & -1.10 \\
$E_{\text {Bragg }}$ & 7.36 & 3.81 & 3.05 & 2.36 \\
$E_{g, L}$ & 1.26 & 1.21 & 1.20 & 1.16 \\
$\phi_{+}$ & 1.60 & 2.11 & 2.44 & 2.68 \\
Dipole step, $\Delta$ & & & & \\
$E_{\text {Bragg }}(\operatorname{expt})$ & -2.41 & -1.31 & -1.40 & -1.57 \\
$E_{g, L}(\operatorname{expt})$ & $5.0 \pm 0.1$ & $2.45 \pm 0.05$ & $1.60 \pm 0.05$ & $0.75 \pm 0.05$ \\
$\phi_{+}$(expt) & $1.6 \pm 0.1$ & $1.50 \pm 0.05$ & $1.30 \pm 0.05$ & $1.05 \pm 0.05$ \\
\hline \hline
\end{tabular}


as the distance from the crystal zero to the top of the electron valence bands. A second important reference level, shown as $E_{\text {vac }}$ in Figure 5.4, is the vacuum energy level - the energy in a vacuum outside the solid. For both positrons and electrons, the vacuum level is separated from the crystal zero by the dipole potential step energy $\Delta=-E_{\text {vac }}$. The dipole step results from the distortion of the electron charge distribution at the surface. Since this energy is electrostatic in origin, it has the same magnitude for positrons and electrons but is opposite in direction. In the rare-gas solids, the vacuum level is below the crystal zero for electrons $(\Delta>0)$ and above it for positrons. The dipole potential step can be obtained from electron band structure calculations and/or experimental measurement where it is defined as $\Delta=\mu_{-}+E_{v}$, where $E_{v}$ is the distance from the top of the electron valence bands to the electron vacuum level. The $\Delta$ values given in Table 5.1 were taken from the electron band structure calculations of Puska and Nieminen (1992), who used the same crystal zero. They should be regarded as reasonable estimates at best.

Gullikson et al. (1988) have measured the energy dependence of the elastic specular reflection of positrons from the (111) surfaces of the face-centered cubic rare-gas solids. In each case, they observed an intense first-order Bragg peak corresponding to the reflection of positrons from the periodic sample when the positron energy is within one of the forbidden energy gaps of the positron band structure. From these Bragg peaks they measured the centre position $E_{\mathrm{Bragg}}$ and the width $E_{g, L}$ of the lowest positron band gap which occurs at the $L$ point on the Brillouin-zone boundary ${ }^{\dagger}$ (see Ashcroft and Mermin 1976). They also estimated the positron work function $\phi_{+}$, which is defined as the energy necessary to remove a positron from the medium (i.e. the energy from the bottom of the positron band to the vacuum level, $\left.\phi_{+}=-\mu_{+}-\Delta\right)$. Their results, all of which are measured with respect to the vacuum level, are summarized in Table 5.1.

The results of the positron band structure calculations are also summarized in $\mathrm{Ta}$ ble 5.1. The positron band structures calculated from the Coulomb potentials consistently give values for $\mu_{+}$and $E_{\text {Bragg }}$ that are too large (i.e. the bands are shifted too high in energy). The large $\mu_{+}$resulted in estimates of $\phi_{+}$that were too small. Conversely, the Schrader potential gave positron bands that were shifted too low in energy. The calculated $E_{\text {Bragg }}$ values were consistently below the experimental ones, while the calculated $\phi_{+}$values were

\footnotetext{
†The point $\Gamma$ is at the center of the Brillouin zone. The names $K, L, W$ and $X$ are widely used for points of high symmetry on the zone boundary. For an fcc lattice: $\Gamma(0,0,0), K(1,1,0), L(0.5,0.5,0.5), W(1,0.5,0)$, and $X(1,0,0)$ in units of $2 \pi / a$, where $a$ is the lattice constant.
} 
all far too large. The $r_{1}$ parameter in the Abarenkov potentials was chosen so that the calculated $E_{\mathrm{Bragg}}$ corresponded to the experimental values to within $0.05 \mathrm{eV}$. This results in $\phi_{+}$that are all larger than the experimental values. The estimates for the first band gap $E_{g, L}$ are largest for the Coulomb potential, smallest for the Schrader potential, and in between for the Abarenkov potentials. Unfortunately, even the Coulomb potential estimates are too small for neon and argon. This is in contrast to Puska and Nieminen (1992) who had Coulomb band gap estimates that were consistently too large, Schrader estimates that were consistently too low, and band gaps calculated from the Abarenkov potential that were in excellent agreement with experiment. Apart from the poorer values for the band gaps, these results are either similar to those of Puska and Nieminen (1992) or in better agreement with experiment.

The positron band structures in Figure 5.4 are all reasonably close to free-particle bands with band gaps at the Brillouin-zone boundary. The energy difference between the bottom of the bands and the center of the first band gap is

$$
E_{\mathrm{Bragg}}-\mu_{+}=\frac{\hbar^{2} k_{L}^{2}}{2 m_{6}^{*}}
$$

where $k_{L}$ is the $\mathbf{k}$ vector at the $L$ point and $m_{b}^{*}$ is the positron band effective mass ${ }^{\dagger}$ given by

$$
m_{b}^{*}=\hbar^{2}\left(\frac{d^{2} E}{d k^{2}}\right)^{-1}
$$

The band effective mass is easily calculated by measuring the curvature around $k=0$ for the lowest energy band in Figure 5.4. Doing this gives $m_{b}^{*}=0.87 m$ for each of the rare-gas solids, where $m$ is the free particle mass. Inserting $m_{b}^{*}$ into (5.7) gives free-particle like predictions for $E_{\mathrm{Bragg}}-\mu_{+}$that are in excellent agreement with the calculated separation (see Table 5.1).

Also shown in Figure 5.4 are the Wigner-Seitz, $E_{\mathrm{ws}}$, and the $\mathbf{k}=0$ pseudopotential energies. The Wigner-Seitz energies were calculated by simply integrating (2.7) using the same positron potentials, with the boundary condition that the positron wave function be flat on the surface of the Wigner-Seitz sphere. The pseudopotentials energies were calculated using the single atom potentials, $v(r)$, and (2.31). The APW energies at $\mathrm{k}=0$

\footnotetext{
${ }^{\dagger}$ The positron effective mass $m^{*}$ contains the contributions from the periodic lattice (band effective mass $m_{b}^{*}$ ) and from the correlated electrons around the positron (correlation effective mass $m_{c}^{*}$ ). The band effective mass can be calculated from (5.8). Calculating the correlation effective mass is a difficult many-body problem that has not yet been solved adequately.
} 


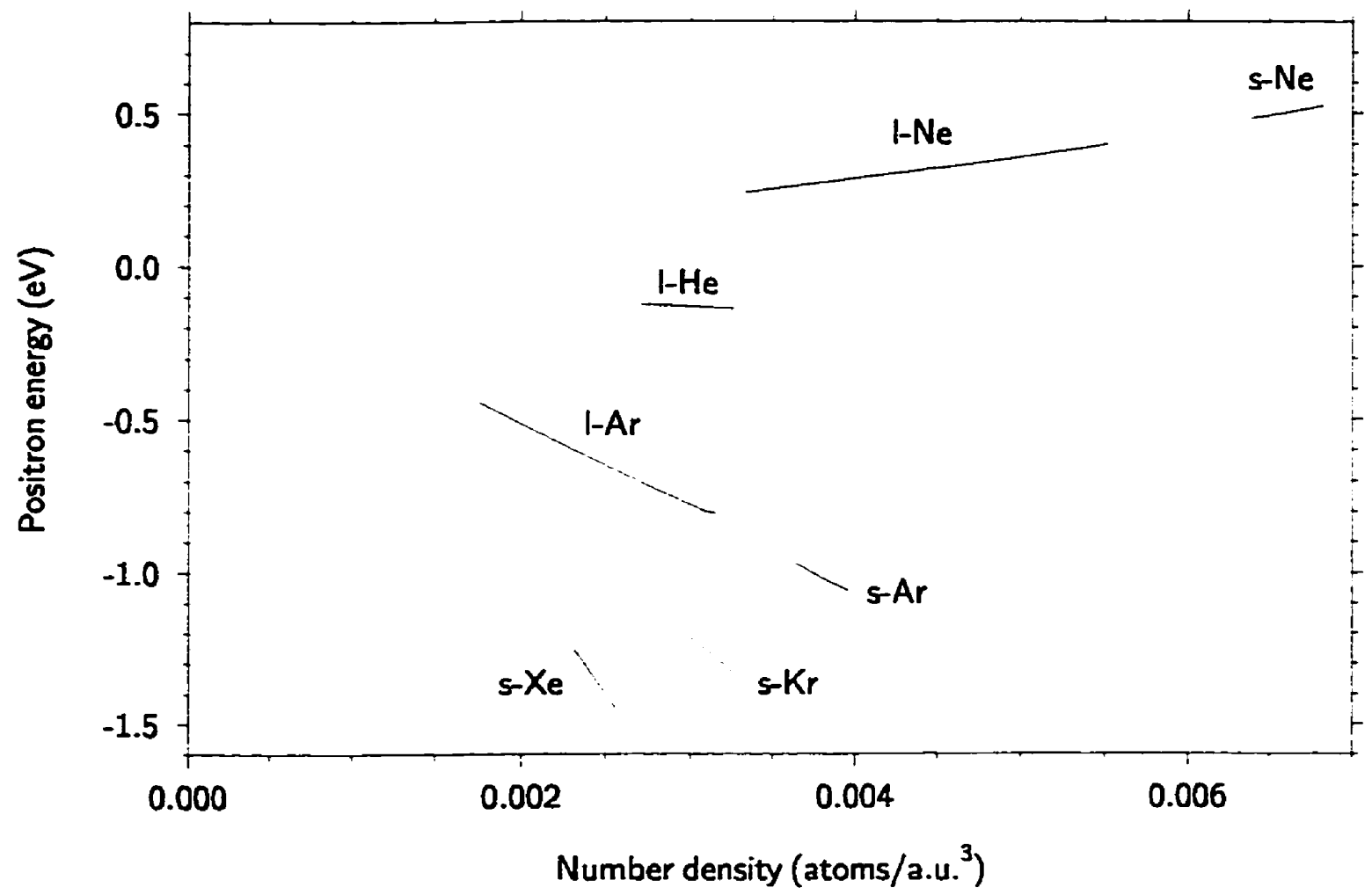

Figure 5.5: The positron ground-state energy in the condensed rare gases as a function of number density.

are about 0.1 to $0.25 \mathrm{eV}$ greater than the Wigner-Seitz and pseudopotential energies. Not shown in Figure 5.4, are the energies of the lowest positron band calculated using the full pseudopotential equation (2.26). The pseudopotential results were very similar to the APW results except that they were consistently lower in energy by an amount equal to the difference at $\mathbf{k}=0$.

Finally, in Figure 5.5 the positron energy at $\mathrm{k}=0$ is plotted as a function of number density $n_{0}$ for the condensed rare gases. The pseudopotential equations (2.31) and (2.32) were used for solids and liquids, respectively. The required lattice constants and liquid densities were taken from Cook (1961). The liquid structure factors were taken from available experimental data (neon: DeGraaf and Mozer (1971); argon: Yarnell et al. (1973)). In all cases the energy is reasonably linear as a function of $n_{0}$ and tends towards zero as $n_{0}$ goes to zero. 


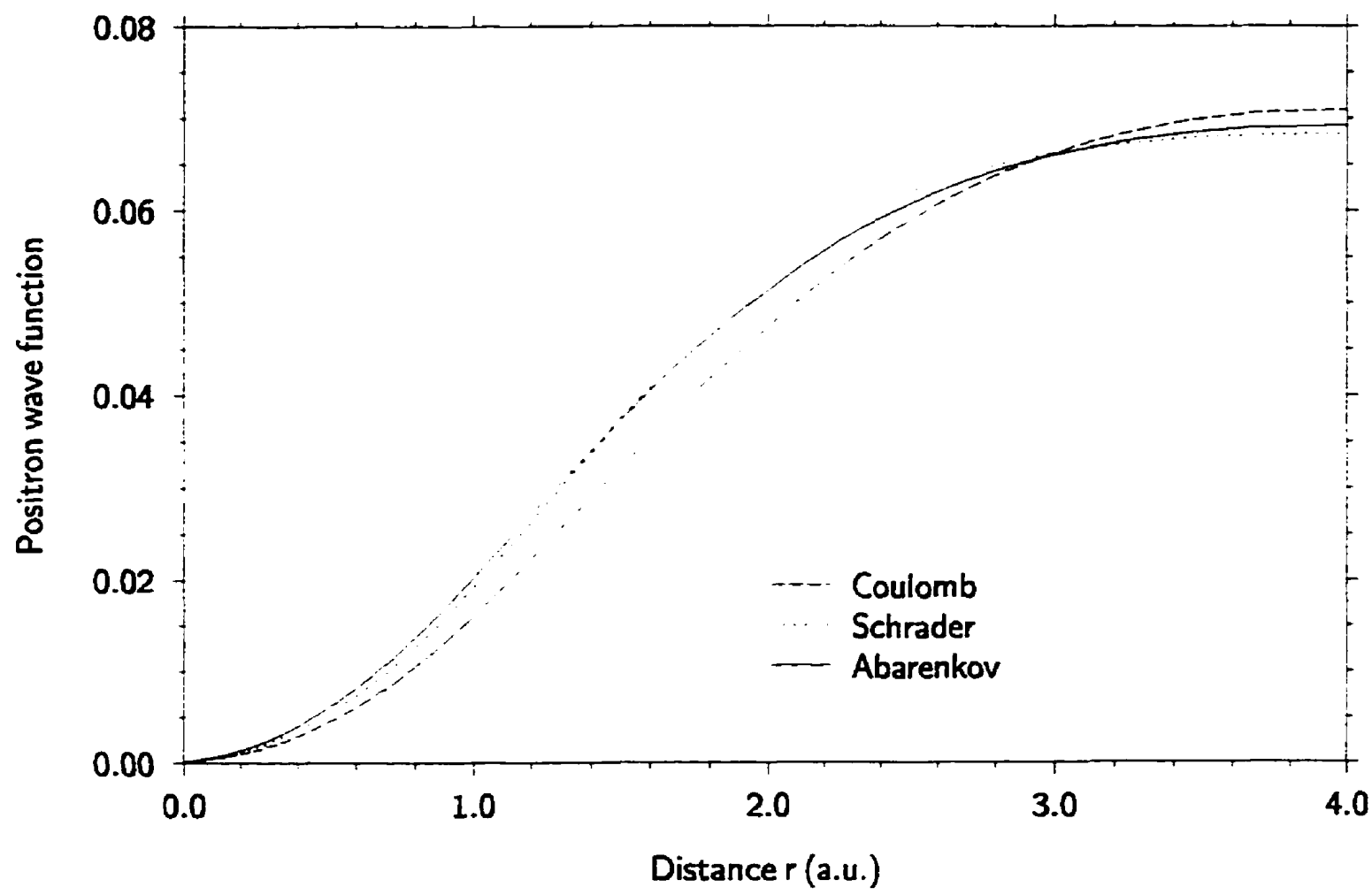

Figure 5.6: Wigner-Seitz positron wave functions for solid argon. The different lines correspond to the potentials shown in Figure 5.3. The Wigner-Seitz radius is at 3.92 a.u.

\subsubsection{Positron Wave Functions}

Using the positron potentials in Figure 5.3 it is also possible to calculate estimates for the solid-state positron wave functions in the rare-gas solids. It is expected that these wave functions will also be reasonable approximations to the positron wave functions around the atoms in the rare-gas liquids as well. Using solid-state wave functions to approximate positron wave functions in the liquid should be valid so long as the long-range disorder in a liquid does not play an important role in determining the positron's annihilation characteristics. Since these characteristics depend mainly on the overlap of the positron and electron wave functions around each atomic site this is expected to be the case. The fact that the broad component in each rare-gas liquid is the same, to within experimental uncertainty, as in the corresponding solid lends support to this approach.

As a first approximation, the Wigner-Seitz method can be used to calculate the $\mathrm{k}=0$ positron wave function around each atom in the lattice. This approach involved integrating the radial Schrödinger equation subject to the boundary condition that the resulting wave 


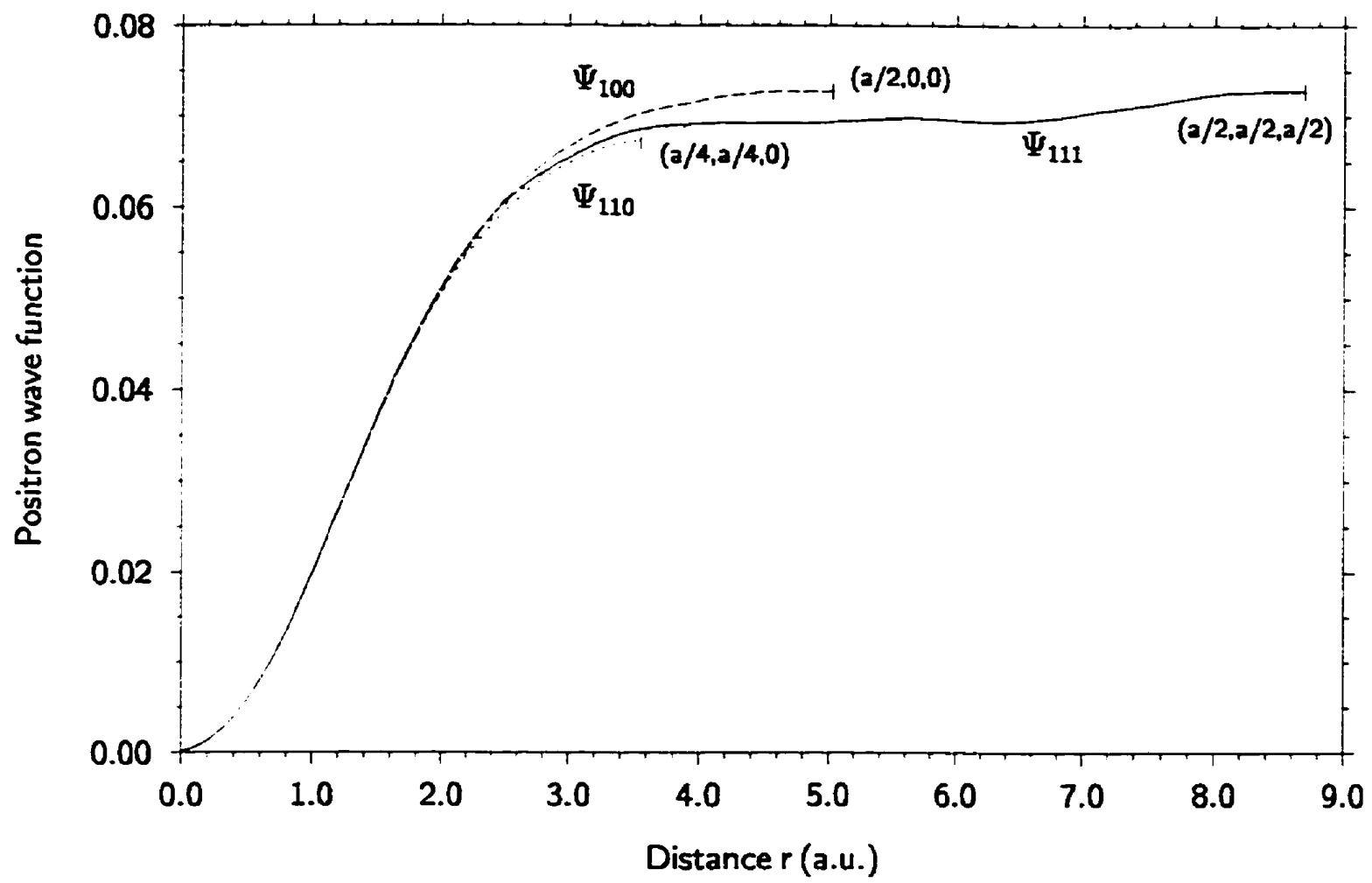

Figure 5.7: Positron wave functions along various directions inside the Wigner-Seitz cell in solid argon.

function be flat at the surface of the Wigner-Seitz sphere. The results for solid argon are shown in Figure 5.7. The dashed, dotted and solid lines correspond to the solution to (2.7) using the Coulomb, Schrader and Abarenkov potentials shown in Figure 5.3, respectively. Each wave function is normalized to one over the unit cell. Notice that the effect of the correlation potentials is to cause the positron wave function to extend slightly more into the centre of the atom as compared to the wave function calculated using the Coulomb potential. The wave function calculated using the Coulomb potential is identical to the one calculated by Woll and Rose (1966). The Wigner-Seitz positron wave functions for the other rare gases are similar.

To gauge the quality of the Wigner-Seitz results, positron wave functions were also calculated using the psendopotential method. This involved first calculating the energy and angle independent positron core factor, $U(r)$ in (2.18), by again integrating the radial Schrödinger equation. This is the very same as the Wigner-Seitz approach except that now the boundary condition must be satisfied at the muffin-tin radius instead of the Wigner-Seitz radius. The resulting core wave functions are similar to the Wigner-Seitz wave functions. 
Now it is possible to use (2.30) to calculate the $k=0$ pseudo-wave functions in various crystal directions. The pseudopotential for the rare gases is very weak, compared to the original potentials. The pseudo-wave functions in each crystal direction were therefore relatively flat and the total positron wave functions each resembled the original core wave function. Total positron wave functions in the [100], [110], and [111] directions in the fcc solid argon, calculated using the Abarenkov potential, are shown in Figure 5.7. The spherical average of the solid-state wave functions in a fcc lattice can be obtained from the averaging formula (Ray and Trickey 1981)

$$
\Psi_{\mathrm{avg}}(r)=\frac{1}{35}\left(10 \Psi_{100}(r)+16 \Psi_{110}(r)+9 \Psi_{11 \mathrm{l}}(r)\right)
$$

The spherically averaged wave function is virtually identical to both $\Psi_{111}$ out to the WignerSeitz radius and to the Wigner-Seitz wave function shown in Figure 5.6. The results for the other rare gases are again similar. Given that the positron wave functions are reasonably similar throughout the Wigner-Seitz cell, the spherically averaged wave function will be used to calculate the positron's annihilation characteristics.

\subsubsection{IPM Results}

Given reasonable positron wave functions, the tight binding model described in $\S 2.5 .3$ can be used to calculate the positron-core electron annihilation characteristics. As a first approximation, the electron-positron correlations will simply be ignored - i.e. $g(r, n l)=$ 1 in (2.45) and (2.47). The rare-gas core electron wave functions were calculated using the same high quality "double-zeta" analytic fits to the Hartree-Fock electron wave functions that were used to calculate the Coulomb potentials (Clementi and Roetti 1974). The positron wave functions were calculated using the Coulomb potentials shown in Figure 5.3. The calculated IPM broad component momentum distributions are shown as dotted lines in Figure 5.8. All of the curves have been arbitrarily normalized to provide a reasonable fit to the data at larger angles (i.e. $\theta_{z}>4.0 \mathrm{mrads}$ ) away from the narrow peak. Only one calculated broad component is shown for each rare gas since the shape of the curve is insensitive to the rare-gas density. In fact, the broad component calculated for the lowestdensity liquid is virtually indistinguishable from that calculated for the solid. The FWHM are 15.3, 10.7, 9.7, and 9.2 mrads for neon, argon, krypton, and xenon, respectively. While these curves remain somewhat wider than the Gaussian estimates to the broad momentum distributions they are narrower that the IPM results of Sharma and Majumdar (1979), 

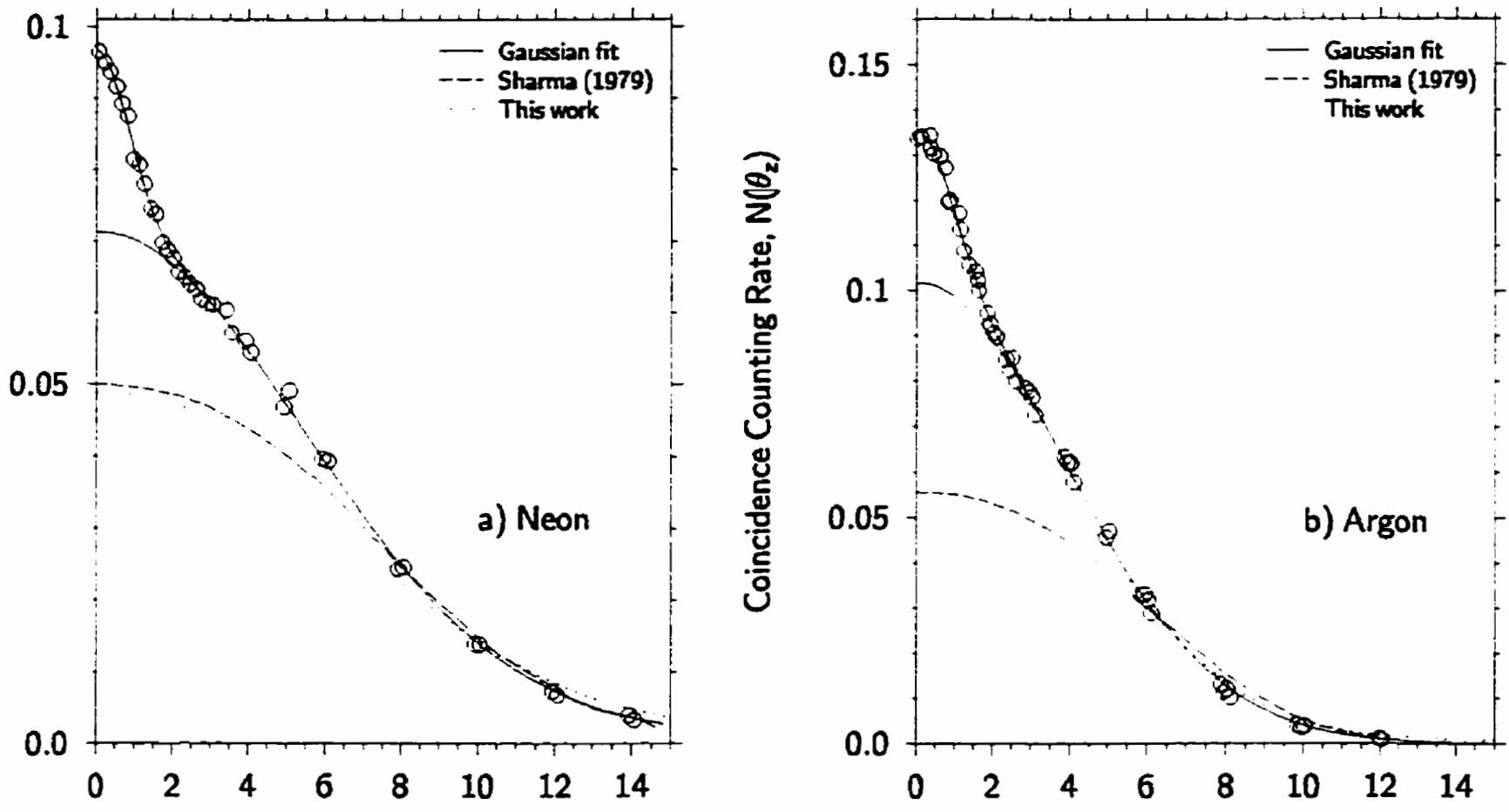

Angle Between Annihilation Photons, $\theta_{2}$ (mrads)
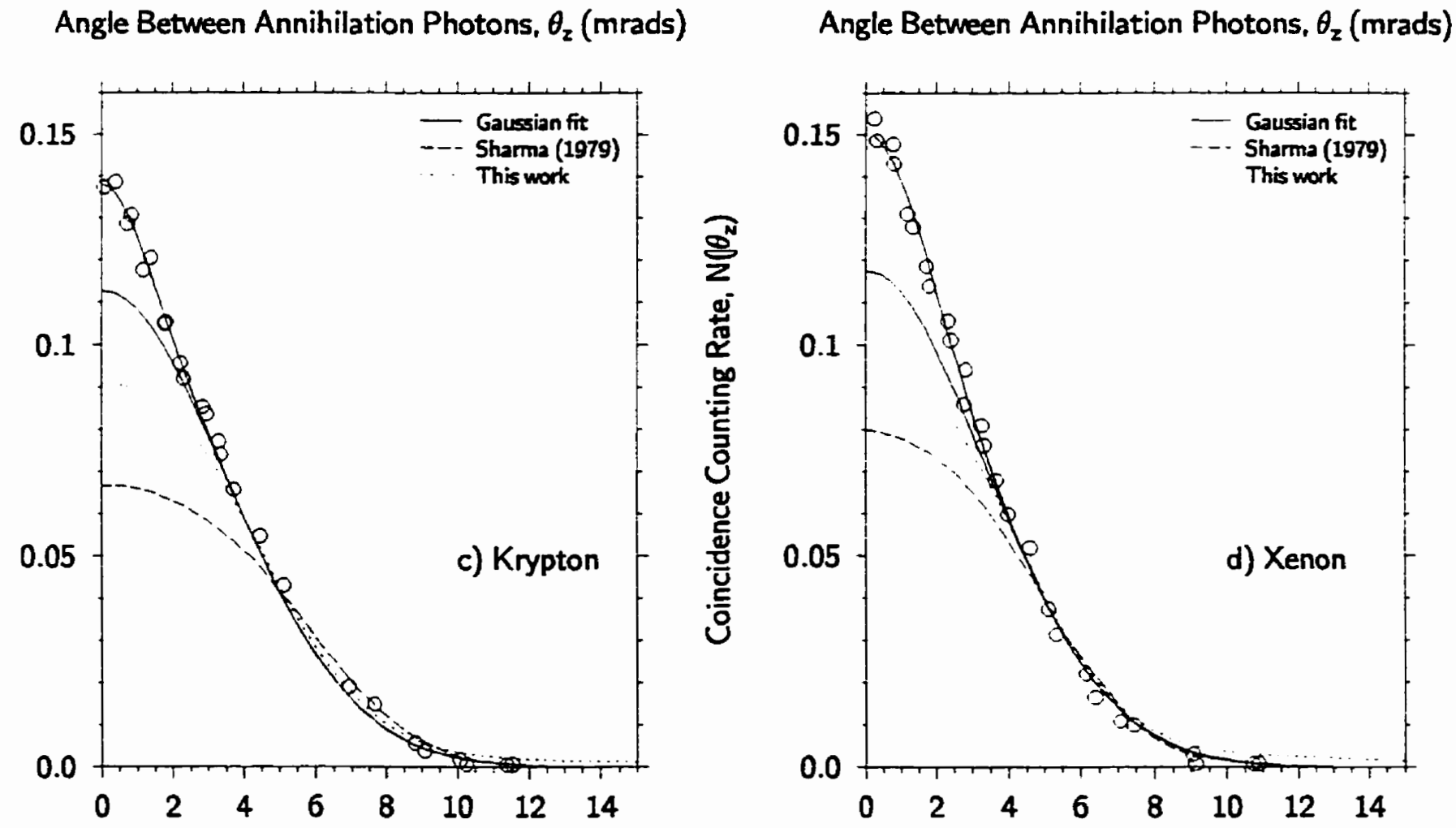

Angle Between Annihilation Photons, $\theta_{2}$ (mrads)

Angle Between Annihilation Photons, $\theta_{\mathbf{z}}$ (mrads)

Figure 5.8: The broad momentum components in the rare-gas solids calculated using the independent-particle model. The dashed lines are the IPM calculation of Sharma and Majumdar (1979). The IPM results of Sharma and Singh (1982) are nearly identical to the dashed lines and are therefore not shown here. The dotted lines are the IPM calculations from this work obtained using the bare Coulomb potential. These lines represent the actual IPM results as no electron-positron correlations are included. 


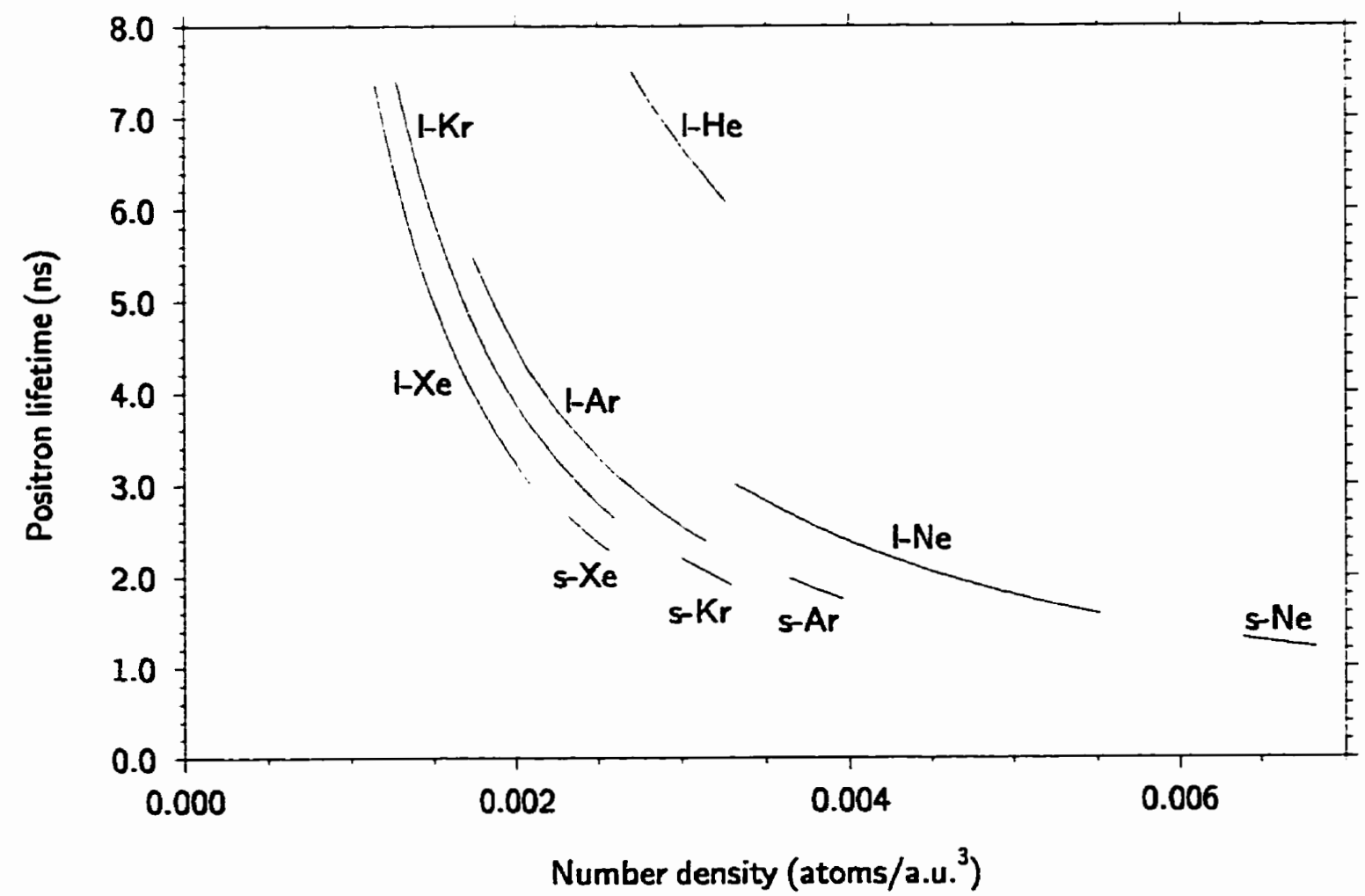

Figure 5.9: The IPM positron lifetimes in the condensed rare gases as a function of number density. 
shown by the dashed lines in Figure 5.8. The shape of the broad component also appears to be reasonably insensitive to the exact shape of the positron potential used to calculate the positron wave functions. Repeating the IPM calculations using the Abarenkov potentials, instead of the Coulomb potentials, to calculate the positron wave functions gives broad components that have nearly the same shape as the dotted lines in Figure 5.8. These are not true IPM calculations since the Abarenkov potentials include some correlation effects - they account for the distortion to the positron wave function caused by the core-electron polarization. However, they do show that the differences between the IPM results of Sharma and Majumdar (1979) and this work cannot be explained by differences in either the rare-gas densities or the positron-rare-gas potentials.

Figure 5.9 summarizes the IPM positron lifetime calculations in the condensed rare gases. The results for the rare-gas solids are slightly less than those of Sharma and Majumdar (1979) who calculated IPM positron lifetimes of $1.51,1.86,2.40$, and 3.15 ns for solid neon, argon, krypton, and xenon, respectively. These results confirm what is already well known - that while the IPM gives a reasonable first approximation to the shape of the broad momentum component, it fails to predict the positron lifetime. In this case, the IPM estimates are 3-6 times the measured positron lifetimes. Also notice that the positron lifetimes, in contrast to the broad momentum component, are very sensitive to changes in the rare-gas density. For the rare-gas solids, a change of $1 \%$ in the lattice constant causes a change of about $5 \%$ in the IPM positron lifetime. This result is consistent with those of Daniuk et al. (1991) who studied the rare-gas core annihilations in a variety of simple and transition metals. The thermal expansion or contraction of a lattice simply changes the overlap between the positron and the core electrons causing a proportional change in the positron lifetime. The effect is even more pronounced in the rare-gas liquids.

\subsubsection{Effects of Positron-Core Electron Enhancements}

To date, most positron studies have simply ignored the positron-core electron correlations, or at best, applied a small, constant, momentum-independent enhancement factor. For the very tightly bound, inner core electrons (ie. those with a momentum $p \geq 15$ mrads) this is probably reasonable. Equation (2.59) estimates an average enhancement factor of $\gamma_{\text {corr }} \sim 1-1.5$ for positron-inner core electron annihilations. However, the IPM calculations above imply average core electron enhancement factors of 3-6 in the condensed rare gases. 
Table 5.2: The average positron-core electron enhancements calculated using (2.53) and the IPM lifetime calculations of this work. These compare quite well with the average enhancements deduced by Sharma and Majumdar (1979). Also shown are the predictions of (5.10). $r_{s}^{o}$ is the value of the arbitrary cutoff parameter introduced into the Jarlborg and Singh enhancement theory. $r_{0}$ is the muffin-tin radius beyond which the electron density was assumed to be constant.

\begin{tabular}{lllll}
\hline \hline & $\mathrm{Ne}$ & $\mathrm{Ar}$ & $\mathrm{Kr}$ & $\mathrm{Xe}$ \\
\hline & 3.1 & 4.6 & - & 5.8 \\
$\gamma_{\text {corr }}(2.53)$ & 3.5 & 4.3 & - & 6.8 \\
$\gamma_{\text {corr }}$ (Sharma and Majumdar 1979) & 3.9 & 6.3 & 7.1 & 8.0 \\
$\gamma_{\text {corr }}(5.10)$ & & & & \\
Cutoff parameter, $r_{s}^{\circ}$ (a.u.) & 2.80 & 3.00 & 3.00 & 3.60 \\
$r_{\text {o }}$ (a.u.) & 1.70 & 2.80 & 3.03 & 3.51 \\
\hline \hline
\end{tabular}

Daniuk et al. $(1989,1991,1992)$ have studied positron annihilations with the ionic rare-gas cores of metals. They observed that at the beginning of each series of metals ( $\mathrm{Na}$ to $\mathrm{Al}, \mathrm{K}$ to $\mathrm{Zn}, \mathrm{Rb}$ to $\mathrm{Cd}$ ) the average core electron enhancement factor for low-momentum outer core electrons (ie. $p<15$ mrads) was significantly larger than for the high-momentum regions. Sob $(1985 \mathrm{a}, \mathrm{b})$ studied the enhancement factors from the ionic rare-gas cores of alkali metals. He deduced a simple relationship to describe the many-body interactions between a positron and core electrons with momenta less than 15 mrads based on the polarizability, $\alpha$, of the rare-gas core:

$$
\gamma_{\text {corr }}=1+1.988 \ln (8.309(\alpha+0.131))
$$

where $\alpha$ is in $10^{-24} \mathrm{~cm}^{3}$. The average positron-core electron enhancement factors calculated using (5.10), and shown in Table 5.2, are all significantly higher than those suggested (2.59) and closer to those deduced from lifetime experiments. While these average enhancement results have no momentum dependence, and therefore no effect on the 1D-ACAR, the fact that they are much higher than the expected high momentum value of 1-1.5 implies that the actual enhancement factors must have a significant momentum dependence for core electrons with momenta below 15 mrads. It is this low-angle momentum dependence of the enhancement factors that is expected to improve the broad momentum component calculations.

Most positron-electron correlation enhancement schemes take into account the effects of the density enhancement of the electrons at the positron site. However, they often 
ignore the distortion of the positron wave function from its IPM value that results from the interaction of the positron with this electron screening cloud. In the condensed rare gases, the electron density enhancement is due to the polarization of core electrons. Its effect on the positron wave function was accounted for by adding a polarization potential to the Coulomb potential in the positron Schrödinger equation, as was done in §5.3.1. Therefore, in the following calculations the more realistic Abarenkov potentials are used to calculate 'correlated' positron wave functions, $\Psi_{p}^{\text {corr }}$. The attractive polarization potential results in positron wave functions that extend slightly further into the center of the atom as compared to the IPM wave functions. This gives a slight increase in the electron-positron overlap, and therefore the momentum density, as compared to the IPM. While inserting the correlated position wave functions into (2.50) made almost no change to the calculated IPM $1 D$-ACAR curves, it did reduce the positron lifetimes estimate by as much as $20 \%$ from the IPM values. However, these lifetime estimates are still far higher than the experimental lifetimes and require that a proper accounting be made of the electron density enhancement at the positron site.

The simplest positron-electron correlation enhancement scheme that can be applied to the rare-gas solids was developed by Chiba et al. (1977). They extended the theory of Salvadori and Carbotte (1969) and obtained two very simple equations, given by (2.57), describing the momentum dependent enhancements - one for localized and another for Bloch electron states. Figure 5.10 shows the effective momentum dependent enhancements, as defined by (2.52), obtained by inserting (2.57) into (2.55). Notice that these enhancements all have a significant momentum dependence. Below $15 \mathrm{mrads}$ the enhancement predicted for Bloch electrons (2.57b), which is always greater than that predicted for localized electrons, is significantly greater than the generally assumed factor of $1-1.5$. The effective enhancement factor is typically about 4 at low angles, declining to about 2-2.5 by $15 \mathrm{~m}$ rads, and falling to below 1.5 at larger angles. Apart from a normalization factor, the two enhancement formulae result in nearly identical estimates for the 1D-ACAR. Furthermore, these enhanced 1D-ACAR curves, which are not shown, provide only a modest improvement over the IPM results shown Figure 5.8. These results are consistent with those observed by Sharma and Singh (1982) whose enhanced 1D-ACAR curves were also nearly identical to their IPM results.

While the Chiba enhancements have little effect on the 1D-ACAR they do have a significant effect on the predicted positron lifetimes. Equation (2.57a) predicts positron 

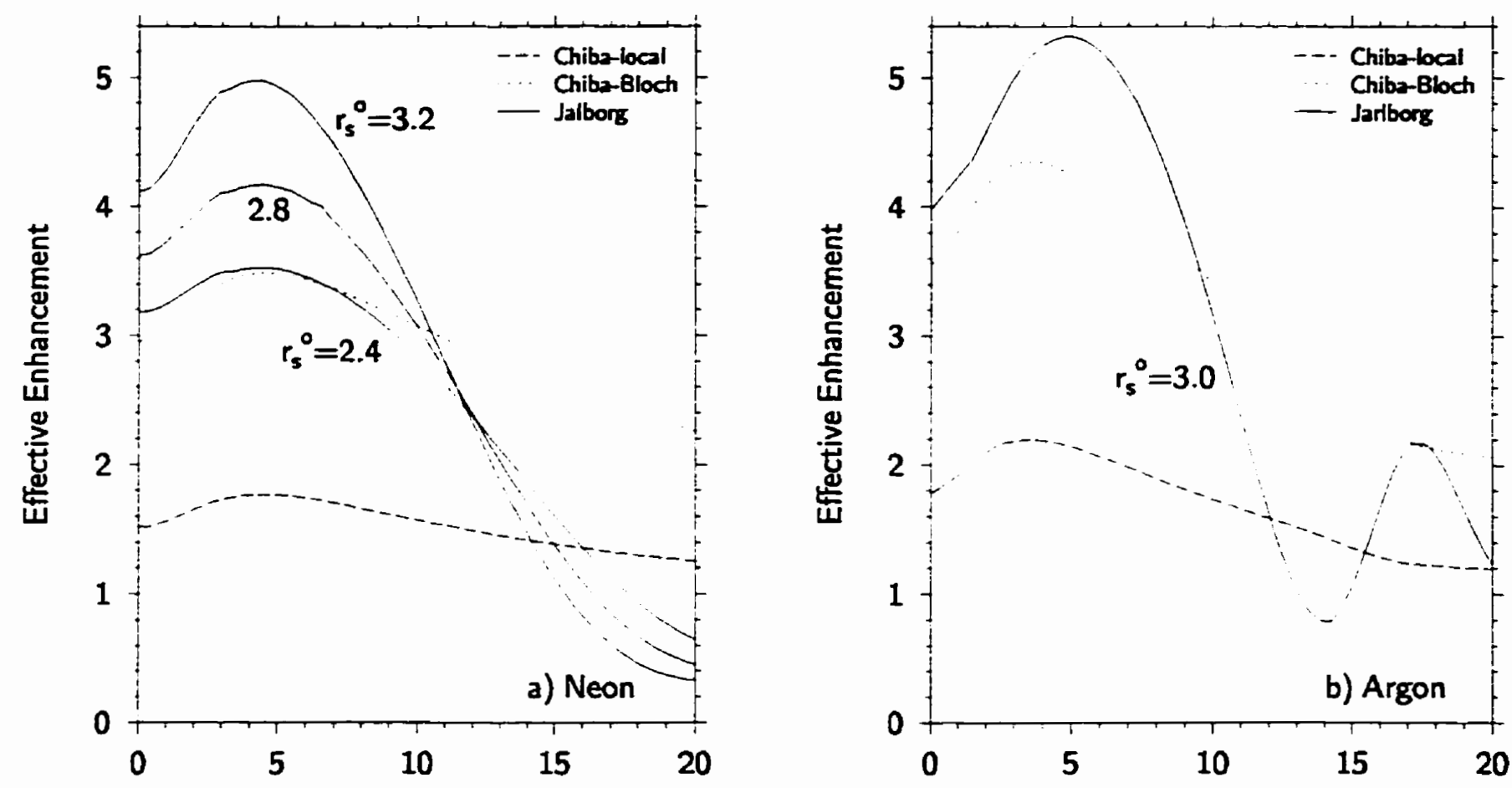

Angle Between Annihilation Photons (mrads)

\section{Angle Between Annihilation Photons (mrads)}
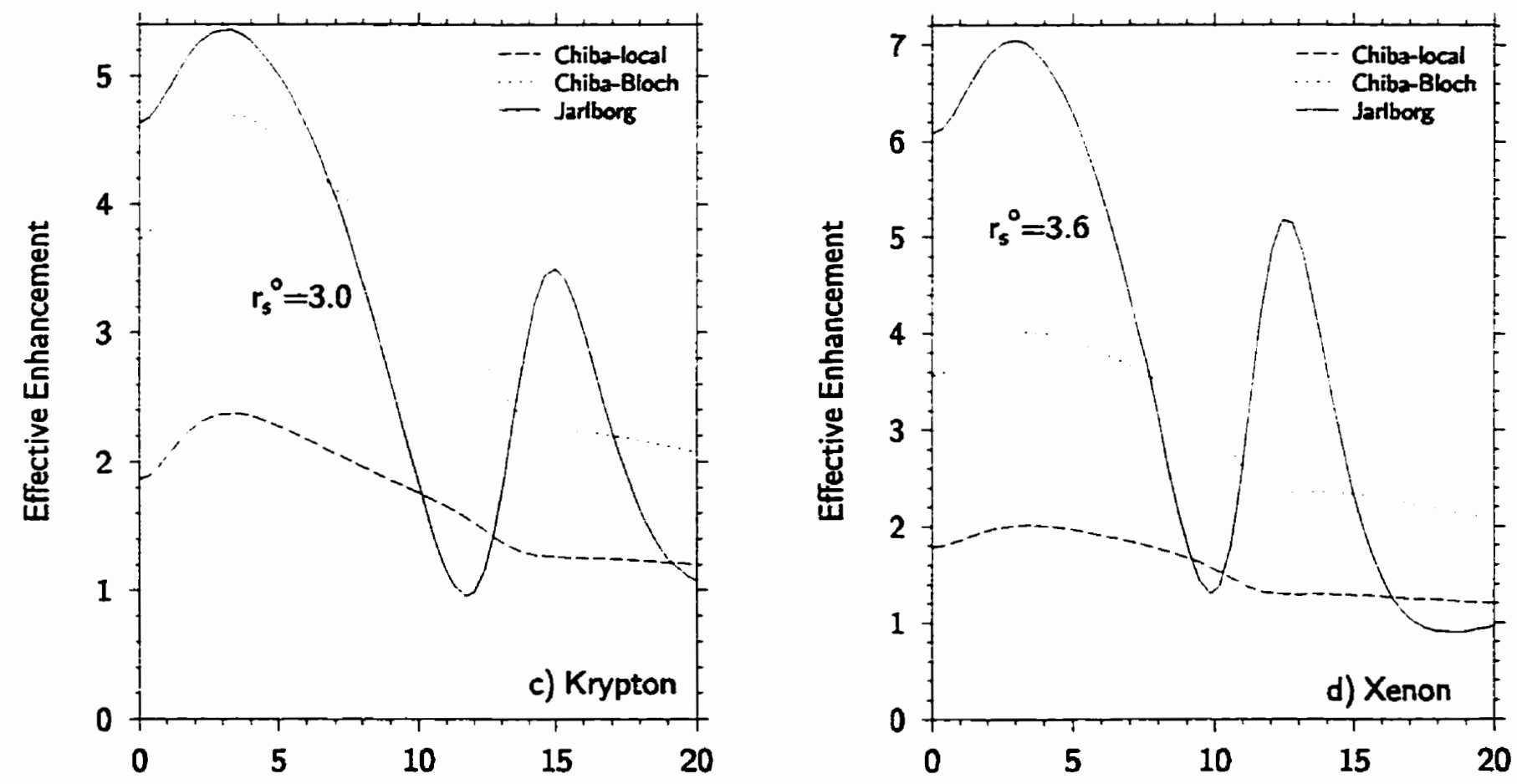

Angle Between Annihilation Photons (mrads)

Angle Between Annihilation Photons (mrads)

Figure 5.10: The momentum dependent positron-core electron correlation enhancements for the rare-gas solids. These enhancements were calculated by simply dividing the enhanced total momentum density by the IPM momentum density as described by (2.52). The dashed and dotted lines were calculated using the enhancement formulae of Chiba et al. (1977) (equations (2.57a) and (2.57b), respectively) to describe the positron-core electron correlations. The solid lines were calculated using the Jarlborg and Singh (1987) enhancement scheme with an additional arbitrary cutoff parameter, $r_{s}^{\circ}$. The effect of the cutoff parameter can be seen in a) where the Jarlborg and Singh enhancement has been calculated using cutoff values of $2.4,2.8$, and 3.2 a.u. 
lifetimes of $0.85,1.02,1.06$, and $1.46 \mathrm{ss}$ for annihilations with localized electron states in solid neon, argon, krypton, and xenon at their normal boiling points, respectively. These predictions are still significantly higher than the experimental results. As expected, the larger enhancement predicted by $(2.57 \mathrm{~b})$ results in shorter positron lifetimes of $0.44,0.51$, 0.54 , and $0.74 \mathrm{~ns}$ for annihilations with Bloch electron states in solid neon, argon, krypton, and xenon, respectively. While these predictions are in much better agreement with those deduced from lifetime experiments, they remain unsatisfactory.

In an attempt to find better agreement with experiment a modified form of the positron-electron correlation enhancements proposed by Jarlborg and Singh (1987) were applied to the rare-gas solids. The enhancements are described by (2.61) in terms of the electron-gas parameter $r_{s}(r)$, described by (2.60). The calculation of the momentum density is straightforward - (2.61) is inserted into (2.43) which is, in turn, inserted into (2.41). The enhancement is calculated at each point in the integration of (2.41) using the same electron densities that were used to calculate the solid-state potentials (see (H.2) in Appendix $H$ ). Unfortunately, doing this calculation results in $1 D$-ACAR spectra that significantly overestimates the broad component at low momenta and the positron annihilation rate. This overestimate is a result of the simplicity of these calculations. The electron densities used to calculate the enhancements are derived from the superposition of atomic potentials. The electron density in the interstitial regions is therefore unrealistically small, giving rise to unrealistically large enhancements. It is expected that proper self-consistent solid-state band structure calculations would give somewhat larger electron densities in the interstitial regions and therefore more realistic enhancements. To cope with this problem, an arbitrary cutoff parameter, $r_{s}^{o}$, was introduced. For $r_{s}<r_{s}^{o}$ the enhancement is simply defined by (2.61). However, for $r_{s}>r_{s}^{o}$ the enhancement was assumed to be constant and equal to the value at $r_{s}^{o}\left(\right.$ i.e. $\left.\epsilon\left(r_{s}^{o}\right)\right)$. This is equivalent to assuming that the electron density has a constant value of

$$
n\left(r_{o}\right)=\frac{3}{4 \pi\left(r_{s}^{o}\right)^{3}} .
$$

outside muffin-tin spheres of radius $r_{0}$ centered around each lattice site.

The cutoff parameter was adjusted to give the best possible fit to both the positron lifetimes and the 1D-ACAR spectra at larger angles away from the narrow peak. Table 5.2 shows the $r_{s}^{o}$ values used in these calculations. Also shown is the actual distance, $r_{o}$, from each lattice site at which the cutoff parameter takes effect. These $r_{0}$ values are about $70-$ 


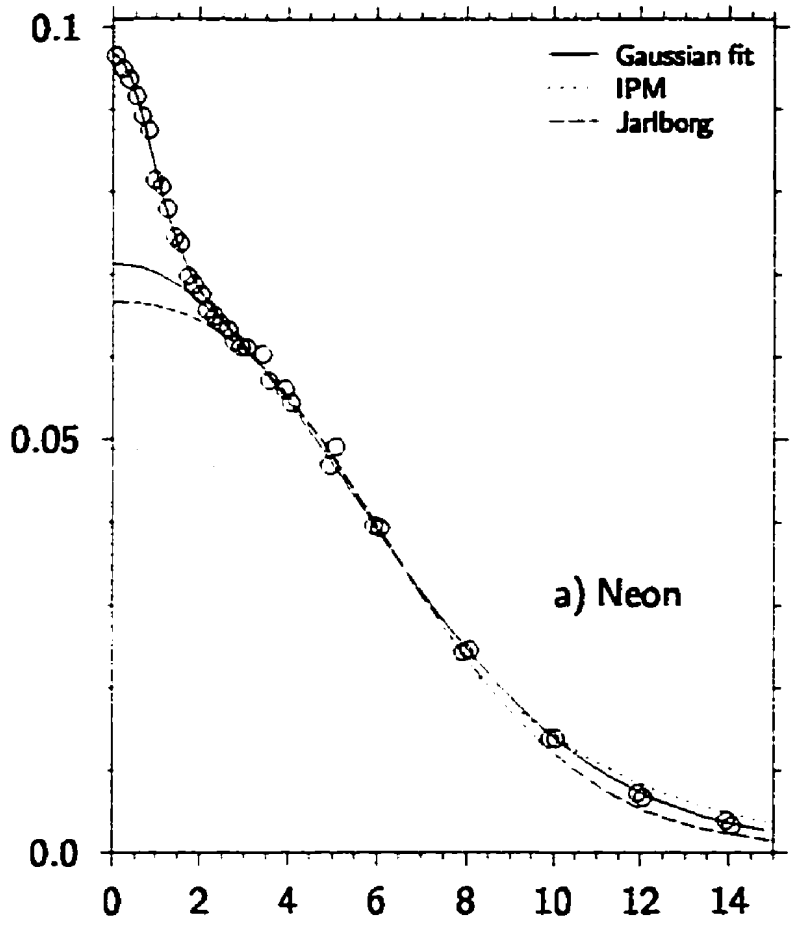

Angle Between Annihilation Photons, $\theta_{2}$ (mrads)

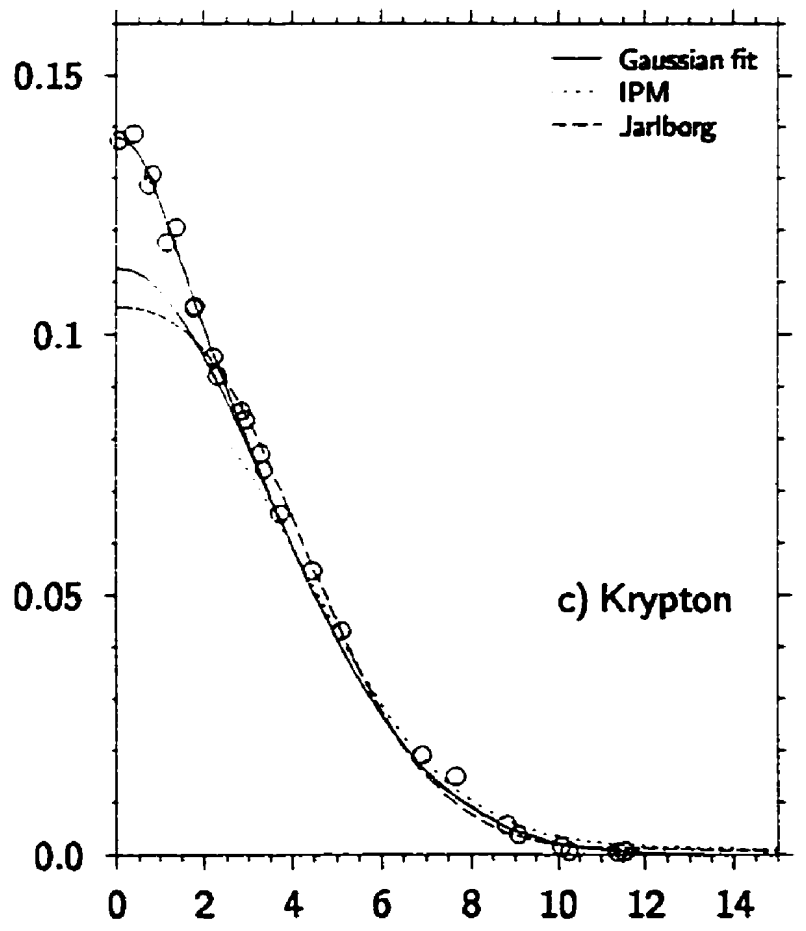

Angle Between Annihilation Photons, $\theta_{2}$ (mrads)

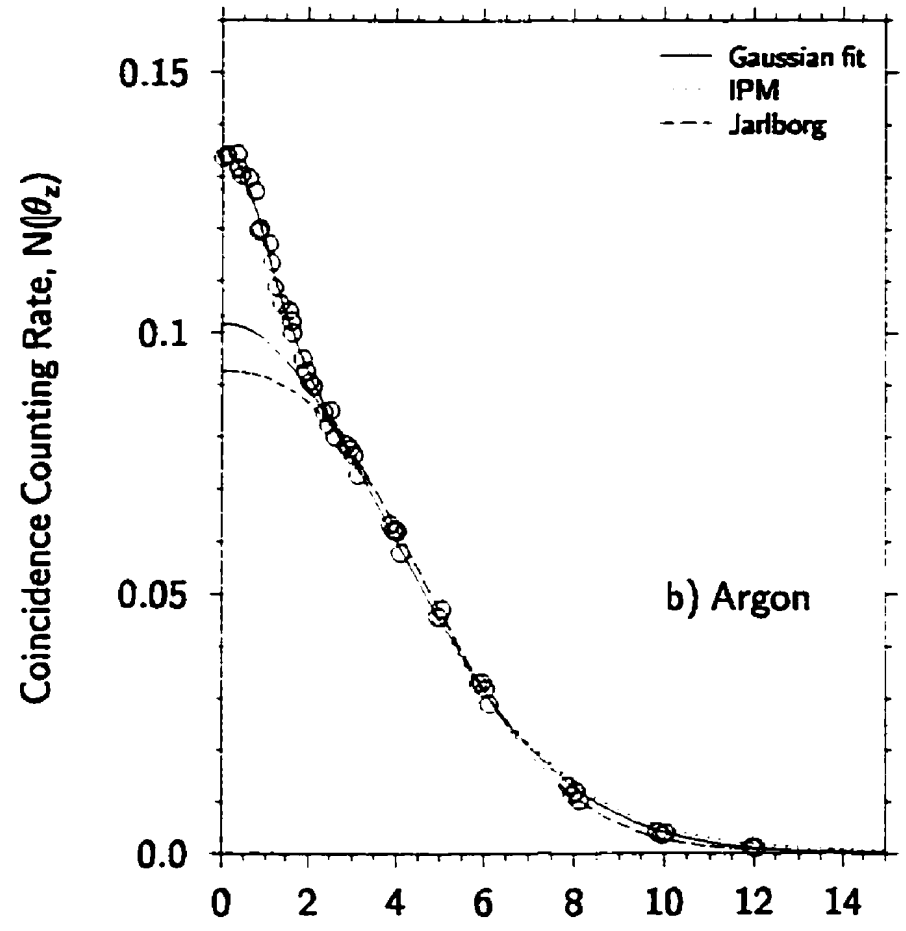

Angle Between Annihilation Photons, $\theta_{z}$ (mrads)

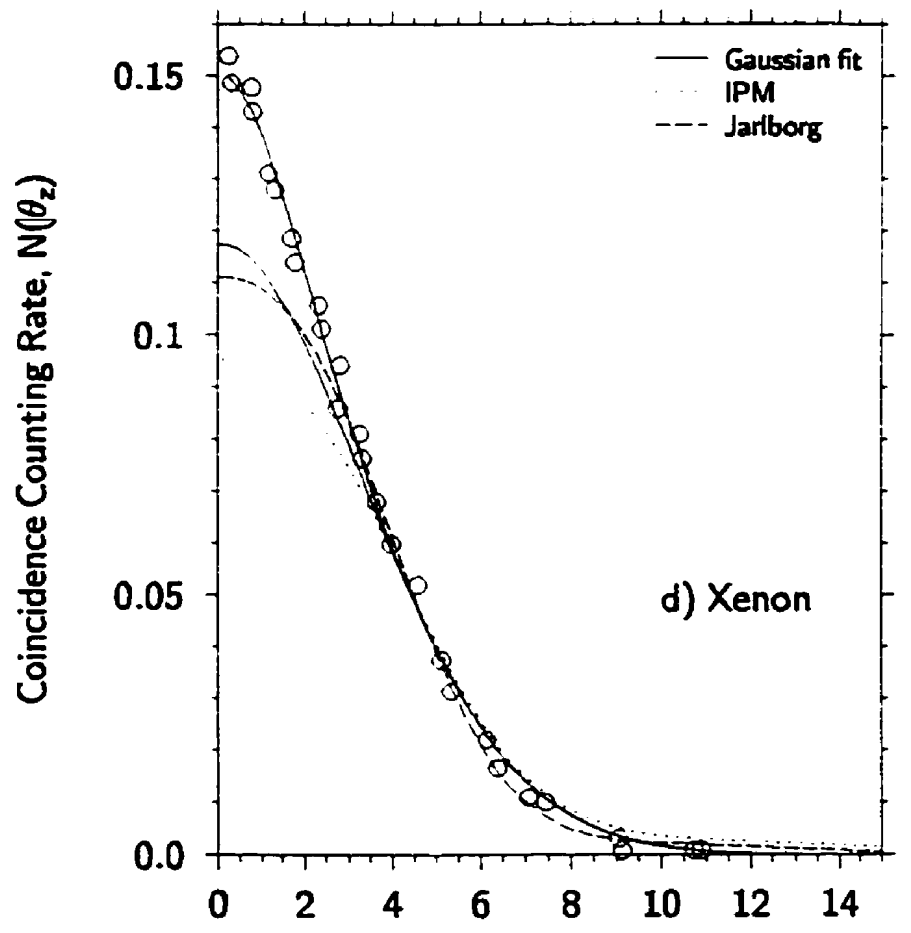

Angle Between Annihilation Photons, $\theta_{\mathbf{z}}$ (mrads)

Figure 5.11: The broad momentum components in the rare-gas solids calculated using the the Jarlborg and Singh (1987) enhancement scheme to model the positron-core electron correlations. The solid lines are the best Gaussian fits to the data while the dotted lines are the previously discussed IPM calculations from this work. 


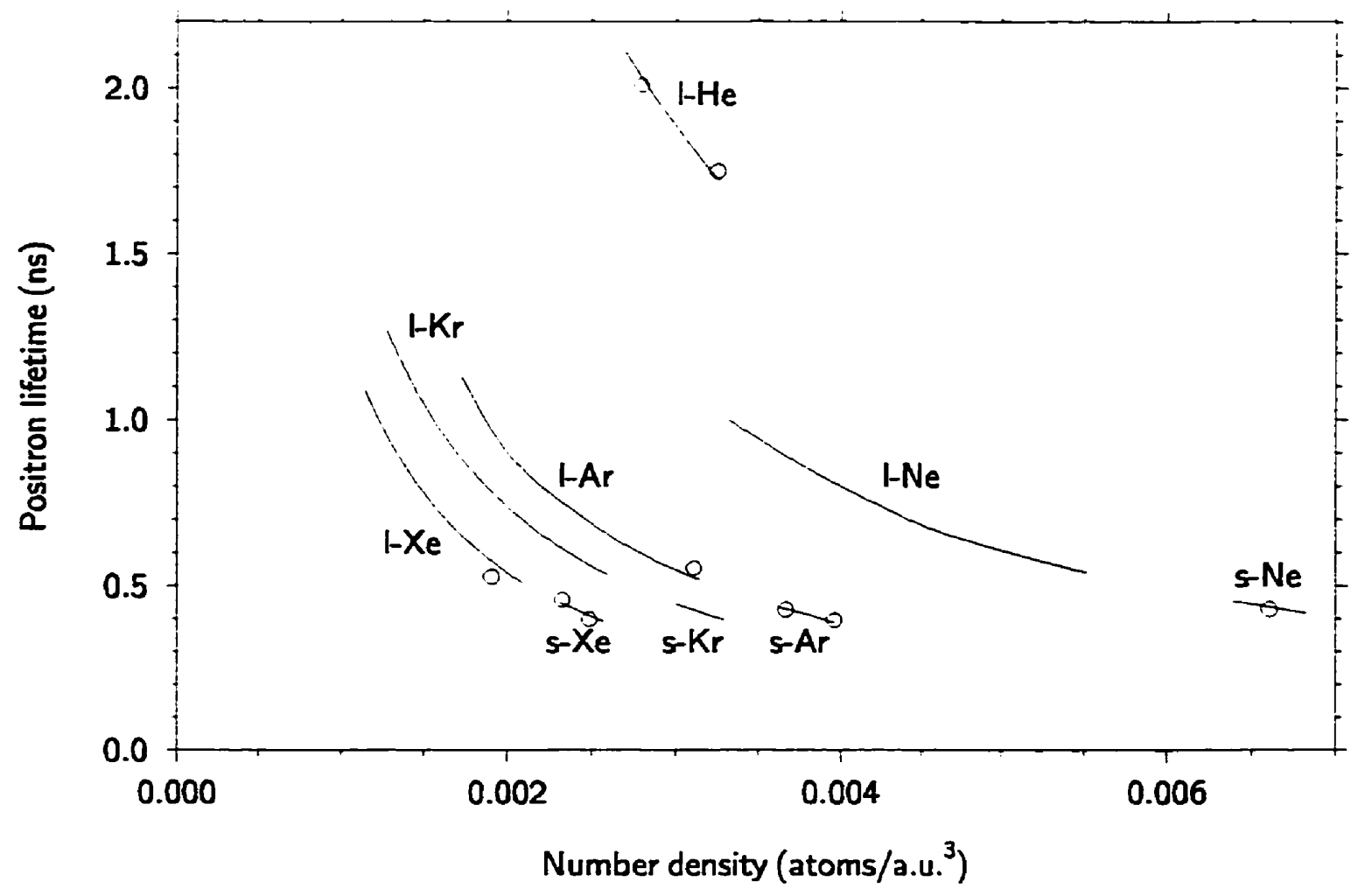

Figure 5.12: The positron lifetimes in the condensed rare gases as a function of number density calculated using the Jarlborg and Singh (1987) enhancement scheme to model the positron-core electron correlations. 
$80 \%$ of the full muffin-tin radii (i.e. half of the nearest neighbour distance in face-centered cubic lattices). The effective enhancement to the calculated momentum density is shown in Figure 5.10. The Jarlborg and Singh enhancements give a momentum density that is significantly narrower and higher than either the IPM or Chiba enhanced results. The behaviour of these enhancements is similar to those calculated by Daniuk et al. (1989), who applied the enhancement scheme of Rubaszek et al. (1984a, 1984b) to the ionic core electrons in the alkali metals. The oscillations in the effective enhancements that occur beyond 10 mrads are unimportant since the momentum density has become negligible at these large angles. Integrating the enhanced momentum density using (4.2) gives the angular correlation spectra shown by the dashed lines in Figure 5.11. In each case, the calculated broad components provide a good fit to the data at larger angles and falls just below the Gaussian estimate of the broad component at smaller angles. Not shown is the ID-ACAR curve calculated for liquid helium. Using a cutoff parameter of $r_{s}^{o}=2.8$ a.u. and treating the liquid as if it were a solid of equal density gives a broad component that is virtually identical to the Gaussian fit defined in Table 4.2 and is as good or better than any of the more sophisticated theoretical calculations. Figure 5.12 shows the positron lifetimes calculated using the Jarlborg and Singh enhancement scheme. The circles represent the best and most reliable experimental lifetime results taken from Table 4.3. 


\section{Chapter 6}

\section{The Narrow Momentum Component}

When positrons enter into condensed insulating media some fraction will form Ps during the slowing down process. In many insulating liquids the repulsive interaction between the medium and the electron in Ps causes it to self-trap into a bubble state. The self-annihilation of $p$-Ps from this bubble state gives rise to the narrow component observed in the angular correlation spectra. In contrast to the broad component, the application of magnetic and electric fields and the deliberate addition of $\mathrm{O}_{2}$ impurity had a significan$t$, and sometimes dramatic, effect on the narrow peak intensity. The implication is that these physical and chemical processes can have a significant effect on the Ps annihilation characteristics. The main aim of this chapter is to gain some insight into these effects.

The chapter starts with calculation of Ps-rare gas atom potentials which are then used to calculate the energy of free Ps in condensed helium, neon and argon in $§ 6.1$. These calculations are useful to predict whether the free or localized bubble state is preferred thermodynamically. In $\S 6.2$ the zero-field Ps formation fraction is analyzed in terms of the Ore gap and spur Ps formation models. In $\$ 6.3$ the spherical square-well bubble model described in $§ 3.4 .2$ is used to determine if the Ps bubble state is energetically favourable and to obtain theoretical estimates for the bubble radii and the shape of the narrow peak. Sections 6.4 and 6.5 discuss the effects of the applied magnetic and electric fields on the Ps yields. The magnetic field results in liquid argon suggest that another quenching mechanism is competing with the magnetic field. It is most likely that spin-exchange collisions are occurring with either $\mathrm{O}_{2}$ impurity or with excess spur electrons. The effects of $\mathrm{O}_{2}$ impurity on the Ps yields in liquid argon and $\mathrm{N}_{2}$ are discussed in $\S 6.6$. Finally, in $\S 6.7$ the Ps spinexchange model developed in §3.5.3 is applied to liquid argon in an effort to explain the anomalous magnetic field quenching that was observed.

\subsection{Positronium Potentials and Delocalized Energies}

It is useful to begin by calculating the bottom of the band energy for free, thermalized Ps. These results can be inserted into (3.27) to predict whether or not Ps bubble states 
are energetically favourable. When bubble states are preferred, the free Ps energy can be used in (3.28) as an estimate of the depth of the square-well potential seen by the Ps.

Any calculation of the free Ps energy first requires a reasonable potential to describe the Ps interactions with the condensed medium. Following Nieminen et al. (1980) and Tuomisaari et al. (1986), each of whom tried several simple potentials, a Ps and an isolated gas atom are assumed to interact via a parameterized Lennard-Jones two body potential

$$
V_{\mathrm{at}}(r)=4 \epsilon\left[\left(\frac{\sigma}{r}\right)^{12}-\left(\frac{\sigma}{r}\right)^{6}\right] \quad
$$

The total Ps potential is a superposition of spherically symmetric potentials about each atomic site.

The parameters $\epsilon$ and $\sigma$ can be fixed by requiring the potential have the proper long-range behaviour and that it correspond to a preselected scattering length. At large distances the potential approaches the London limit and has the asymptotic condition

$$
V_{\text {at }}(r) \propto-\left(\frac{\alpha_{1} \alpha_{2} I_{1} I_{2}}{I_{1}+I_{2}}\right) \frac{1}{r^{6}}
$$

where the $\alpha$ 's and I's are the polarizabilities and ionization potentials of the two atoms. Given that the proportionality constant can be determined, (6.2) fixes the product $4 \epsilon \sigma^{6}$ in (6.1) since $V_{\text {at }}(r) \rightarrow-4 \epsilon \sigma^{6} / r^{6}$ for large distances. Barker and Brandsen (1968) calculated the asymptotic form for the Ps-He potential to be (in atomic units)

$$
V_{\mathrm{at}}(r)=-\frac{19.3}{r^{6}}
$$

Using this as a reference, along with the rare-gas atom polarizabilities and ionization potentials in Table A.1, a Ps atom polarizability of $5.33 \AA^{3}$ (36a.u. $\left.{ }^{3}\right)$ and an ionization energy of $6.8 \mathrm{eV}$ a proportionality constant of $1.99 \mathrm{a} . \mathrm{u}$. can be calculated for (6.2). This fixes the product $4 \epsilon \sigma^{6}$ to $19.3,36.1,137.7,200.8$ and 311.7 a.u. for $\mathrm{He}, \mathrm{Ne}, \mathrm{Ar}, \mathrm{Kr}$ and $\mathrm{Xe}$, respectively. This leaves only one adjustable parameter for the Lennard-Jones potential.

Given a potential, it is possible to calculate the Ps-atom scattering length associated with it. The scattering length is defined as the s-wave $(l=0)$ phase shift, $\delta_{0}(k)$, in the low energy limit

$$
a_{s}=-\lim _{k \rightarrow 0} \frac{\tan \left(\delta_{o}(k)\right)}{k}
$$




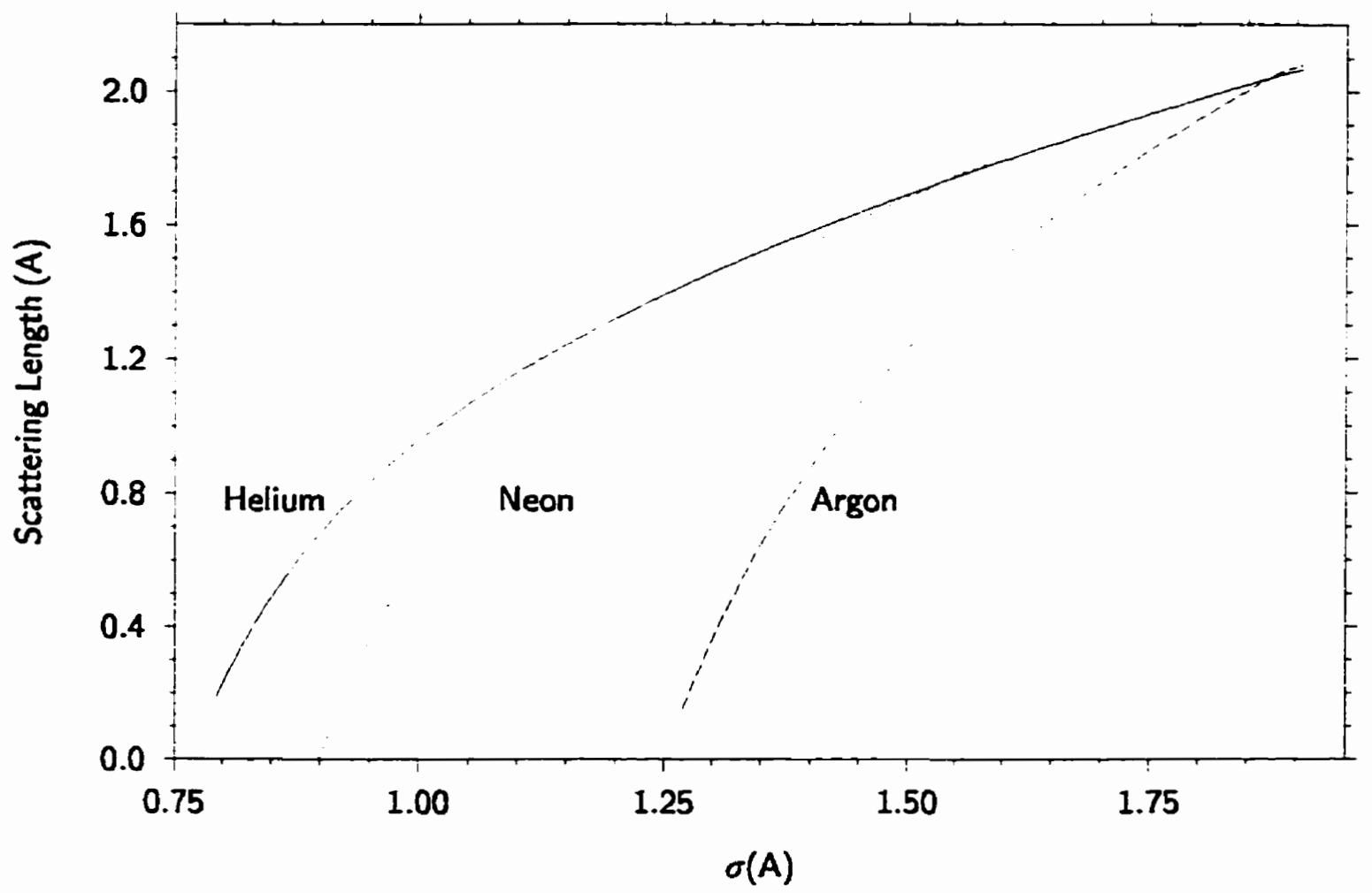

Figure 6.1: The dependence of the Ps-atom scattering length for helium, neon and argon on the Lennard-Jones parameter $\sigma$ defined by (6.1). A preselected scattering length fixes the value of $\sigma$. Using this with the asymptotic condition (6.2) fully specifies the Ps-rare gas atom pair potential.

Table 6.1: The Lennard-Jones positronium-rare gas potential parameters. For helium, the most recent scattering length value of $0.75 \pm 0.05$ measured by Rytsola et al. (1984b) was used to determine the Lennard-Jones parameters. The only known value for the PsNe scattering length is a theoretical estimate of $0.87 \AA$ (Iakubov and Khrapak 1978) which corresponds to to a $\sigma$ of $1.47 \AA$ and an $\epsilon$ of $0.27 \mathrm{eV}$.

\begin{tabular}{llll}
\hline \hline & He & Ne & Ar \\
\hline Ps-atom scattering length $(\AA)$ & 0.75 & 0.87 & 0.79 \\
$\sigma(\AA)$ & 1.27 & 1.47 & 1.60 \\
$\epsilon(\mathrm{eV})$ & 0.35 & 0.37 & 0.62 \\
\hline
\end{tabular}




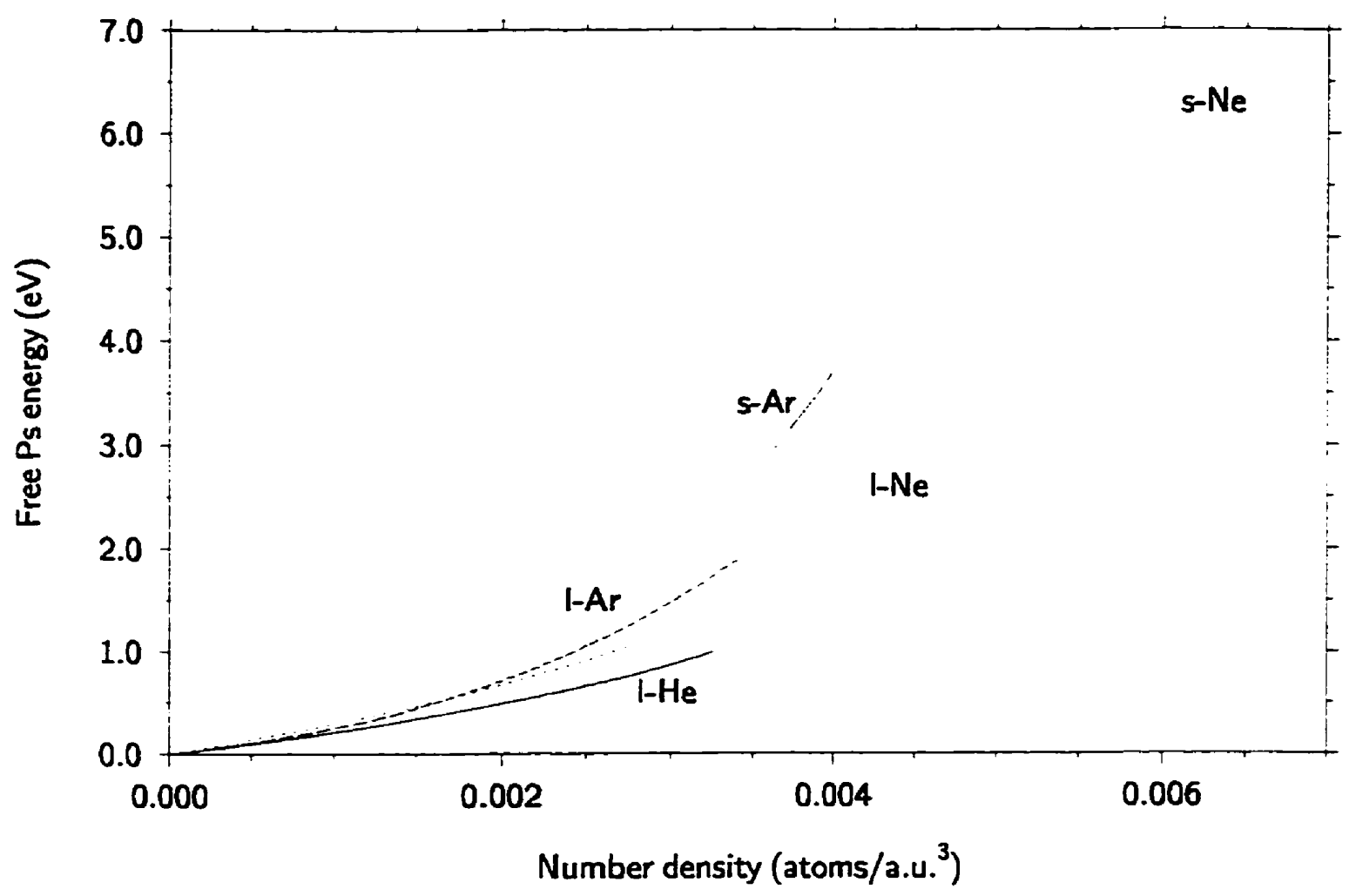

Figure 6.2: The calculated energy of a free Ps in condensed helium, neon and argon as a function of the number density.

The phase shift was calculated by solving the radial Schrodinger equation using the Numerov technique with the following boundary conditions

$$
\Psi_{\mathrm{Ps}_{\mathrm{s}}}(k, r)= \begin{cases}0 & r=0 \\ \frac{1}{k r}\left(\sin (k r)+\tan \left(\delta_{0}(k) \cos (k r)\right)\right. & r \rightarrow \infty\end{cases}
$$

Figure 6.1 plots the calculated scattering lengths as a function of the Lennard-Jones parameter $\sigma$ (i.e. where the potential crosses over from being repulsive to attractive). The most recent theoretical and experimental estimates for the Ps-He scattering length range from 0.50 to $0.80 \AA$ (Hautojarvi et al. 1976; Smith et al. 1977; Rytsola et al. 1984). Therefore, $\sigma$ varies from 0.99 to $1.34 \AA$ and $\epsilon$ from 1.53 to $0.25 \mathrm{eV}$. The only known value for Ps-Ne scattering length is a theoretical estimate of $0.87 \AA$ by Iakubov and Khrapak (1978). This corresponds to a $\sigma$ of $1.47 \AA$ and an $\epsilon$ of $0.27 \mathrm{eV}$. In argon, the Ps scattering length estimates range from 0.53 to $0.79 \AA$ (Tuomisaari et al. 1986) which gives a $\sigma$ between 1.44 and $1.60 \AA$ and an $\epsilon$ between 1.17 and $0.62 \mathrm{eV}$. Table 6.1 summarizes the Lennard-Jones parameters used in these calculations.

Figure 6.2 shows the bottom of the band Ps energy in condensed helium, neon 
and argon calculated using the psuedopotential method described in \$2.4.1.3. In these calculations the strong $r^{-12}$ repulsion in the Lennard-Jones potentials led to a numerical divergence in the $B(k)$ term in (2.31) and (2.32). Since the effect of this term is small it was simply ignored. Notice that at low densities the energy curves all tend to their optical limit

$$
E_{o}\left(n_{o}\right)=\frac{2 \pi \hbar}{m_{\mathrm{Ps}}} a_{s} n_{o}
$$

At higher densities, the multiple scattering from the rare gas atoms and the decreasing free volume leading to a nonlinear density dependence for the energy. The low density portion of the curves for helium and argon are in good agreement with the previous calculations by Nieminen et al. (1980) and Tuomisaari et al. (1986).

One important result of these calculations is that delocalized Ps is not expected to form in a perfect neon or argon lattice. The free energy shown in Figure 6.2 is greater than the Ps binding energy of $4.5 \mathrm{eV}$ in neon and $2.8 \mathrm{eV}$ in argon (Gullikson et al. 1988). Any Ps that formed in these solids would quickly break up again in subsequent collisions. However. the angular correlation experiments in solid neon and argon imply that Ps does exist in these solids. Both solids had a small narrow peak extending above the assumed broad component corresponding to $p$-Ps annihilating from a localized state. As with positrons, it is unlikely that Ps could self-trap in these solids even if it was energetically favourable to do so because it is unlikely that required ionic rearrangements could occur with Ps lifetime. It is therefore most reasonable to assume that either delocalized Ps forms and then quickly finds a suitable vacancy-type defect or that the Ps forms inside a pre-existing defect.

\subsection{Zero-Field Positronium Formation}

Table 6.2 summarizes the 1D-ACAR narrow peak parameters measured in zero electric and magnetic field. These results were obtained by subtracting the broad components defined by the Gaussian fits in Table 4.2 from the total 1D-ACAR curves. The theoretical estimates for the helium broad component are of good quality and the components are distinct enough that broad and narrow can be separated with good confidence. However, for the other condensed gases the separation of the components is somewhat more subjective and less precise process. The Gaussian fits were therefore used for simplicity. While this choice for the broad component may affect the quantitative results, the calculations in 
Table 6.2: A summary of the measured narrow peak parameters. The narrow peak was obtained by subtracting the Gaussian fits to broad component described in Table 4.2 from the total ID-ACAR curves. $\theta_{n}$ is the FWHM of the narrow peak. $I_{n}$ is the narrow peak intensity - i.e. the fraction of area of the total curve that is under the narrow peak.

\begin{tabular}{lccc}
\hline & $\mathrm{T}(\mathrm{K})$ & $\theta_{n}(\mathrm{mrads})$ & $I_{n}(\%)$ \\
\hline $\mathrm{He}(\mathrm{l})$ & 4.6 & $0.79 \pm 0.05$ & $5.1 \pm 0.3$ \\
$\mathrm{Ne}(\mathrm{s})$ & 12.3 & $2.60 \pm 0.07$ & $3.4 \pm 0.5$ \\
$\mathrm{Ne}(\mathrm{s})$ & 23.5 & $2.18 \pm 0.10$ & $4.1 \pm 0.5$ \\
$\mathrm{Ne}(\mathrm{l})$ & 25.3 & $1.86 \pm 0.07$ & $5.1 \pm 0.5$ \\
$\mathrm{Ar}(\mathrm{s})$ & $\sim 83$ & $1.6-3.5$ & $0.3-2.0$ \\
$\mathrm{Ar}(\mathrm{l})$ & 87.0 & $2.1_{-0.2}^{+0.2}$ & $7.3 \pm 0.5$ \\
$\mathrm{~N}_{2}(1)$ & 77.4 & $2.15 \pm 0.20$ & $8.6 \pm 0.7$ \\
& 77.4 & $1.9_{-0.1}^{+0.2}$ & $5.1 \pm 0.7$ \\
\hline
\end{tabular}

§5.3.5 suggest that the Gaussian fits are good enough that the qualitative conclusions will not be affected.

The narrow peak results for liquid helium are in excellent agreement with the results of previous authors shown in Table 4.1. A rough check on the validity of the assumption that the narrow peak in the angular correlation curves is due to $p$-Ps annihilations can be made by comparing $I_{n}$ to the $o$-Ps intensity, $I_{3}$, measured in lifetime experiments. Since p-Ps and o-Ps form in a 1:3 ratio it follows that in the absence of any quenching or spin conversion mechanisms that $I_{n}$ in Tables 4.1 and 6.2 should be one third of the $I_{3}$ value in Table 4.3. In helium, the narrow peak intensity of $5.1 \%$ compares nicely with the long lifetime $o$-Ps intensity of $\sim 15 \%$.

The narrow peak parameters for liquid neon and argon are also in good agreement with previous results. The narrow peak intensity of $5.1 \%$ in liquid neon is consistent with the upper bound of the Ore gap formation model, $F_{\max }$, in Table 6.3 estimated using (3.19) and the data in Table A.5. This implies that nearly all of the positrons that touch down inside the Ore gap form Ps - i.e. very few positrons scatter below the Ps formation threshold energy. No lifetime measurements exist for liquid neon so it is unknown if $I_{n}$ and $I_{3}$ are in a 1:3 ratio. Magnetic field results described below suggest they are. However, this is certainly not the case for the other liquids shown in Tables 4.1,6.2 and 4.3. Especially interesting is liquid argon where $I_{n}=7.3 \%$ and $I_{3}=7.0 \%$ are nearly the same. The narrow 
peak intensity, taken by itself, implies a total Ps formation fraction of about $29 \%\left(4 I_{n}\right)$, which significantly exceeds the predicted Ore gap maximum of $20 \%$. Similarly, the long lifetime intensity, taken in isolation, would predict a narrow peak of only $2.3 \%$ and a total Ps intensity of about $9 \%\left(\frac{4}{3} I_{3}\right)$. The discrepancy between these measurements is the result of various quenching and/or spin conversion mechanisms occurring in liquid argon that do not occur in liquid helium and neon.

Varlaskin (1971) measured narrow peak intensities of $8.2 \%$ and $11.8 \%$ in liquid krypton and xenon, respectively. Unfortunately, there are no lifetime measurements in these liquids. As in liquid argon these intensities suggest a total Ps intensity that exceeds the estimated maximum for the Ore gap formation fraction. It is likely that the same quenching and/or spin conversion mechanisms at work in liquid argon are also present in these liquids.

The narrow peak in solid neon and argon is seen here for the first time. The increase in the narrow peak with magnetic field described below confirms that the peaks are real. The large variations in $\theta_{n}$ and $I_{n}$ and the inconsistency of the results between different samples are likely a consequence of differences in the uncontrolled growing conditions which would result in significantly different defect concentrations and sizes. Varlaskin's (1971) angular-correlation experiment in solid argon did not show a narrow component. In fact, his total 1D-ACAR is virtually identical to the broad component used in Table 4.2. It is likely that his samples were grown more carefully and had a minimum of defects.

The results in liquid nitrogen are interesting and can be used to highlight the difficulties that can arise when a reasonable broad component subtraction is not available. The lack of an angular correlation measurement in solid nitrogen makes it difficult to establish an upper bound for the broad component in the low angle region of the $1 D$-ACAR for liquid $\mathrm{N}_{2}$. Using the best fit Gaussian for the broad component gives a narrow peak intensity of about $8.6 \%$. Lifetime experiments measure a long-lived component intensity of about $15.2 \%$. The lack of a $1: 3$ ratio for $I_{n}: I_{3}$ makes it reasonable to expect that quenching and/or spin conversion mechanisms are at work in liquid $\mathrm{N}_{2}$ as well. However, if the arbitrary and more peaked dashed line in Figure 4.12 is used as the broad component then the narrow peak intensity falls to $5.1 \%$. This result now implies that no quenching or spin-conversion mechanisms are present. This ambiguity does not exist in liquid argon. The ID-ACAR in solid argon defines an upper bound for the broad component in liquid 
Table 6.3: A summary of the Ore gap formation fraction calculations. $E_{l}$ and $E_{u}$ are the lower and upper Ore gap boundary energies, respectively. $E_{x}$ is the inelastic excitation threshold energy. $F_{\min }$ and $F_{\max }$ are the lower and upper Ore gap estimates for the Ps formation fraction estimated using (3.19).

\begin{tabular}{cccccc}
\hline \hline & $E_{l}(\mathrm{eV})$ & $E_{x}(\mathrm{eV})$ & $E_{u}(\mathrm{eV})$ & $F_{\min }(\%)$ & $F_{\max }(\%)$ \\
\hline $\mathrm{Ne}$ & 17.1 & & 21.6 & & 20.9 \\
$\mathrm{Ar}$ & $11.4 \pm 0.1$ & 12.1 & 14.2 & 4.0 & 19.8 \\
$\mathrm{Kr}$ & $9.9 \pm 0.1$ & 10.25 & 11.6 & 2.9 & 14.6 \\
$\mathrm{Xe}$ & $7.9 \pm 0.1$ & 8.36 & 9.3 & 5.1 & 15.5 \\
$\mathrm{~N}_{2}$ & $11.3 \pm 0.5$ & $8.38 \pm 0.01$ & 15.1 & 0 & 25.2 \\
\hline
\end{tabular}

argon that makes it impossible to significantly reduce the current $I_{n}$ result.

In the rare-gas liquids it is likely that Ps is largely formed via the Ore gap mechanism. After their last ionizing collision, positrons in the monoatomic rare gases have relatively few modes of thermalization. Positrons falling below the inelastic excitation threshold must thermalize by phonon scattering in solids or by long-wavelength density fluctuation scattering in liquids. These thermalization processes are inefficient and imply that positrons falling within the Ore gap are effectively 'hung up' there with a high probability of forming Ps. Positrons falling below or bypassing the Ore gap continue to thermalize but only very slowly. Gullikson and Mills (1986) have estimated that below the inelastic excitation threshold the average positron energy loss per collision, $\delta E$ in Table A.5, is only $6 \pm 0.2$ $\mathrm{meV}$ in solid $\mathrm{Ar}$ and $3 \pm 1 \mathrm{meV}$ in solid $\mathrm{Kr}$ and $\mathrm{Xe}$. These values are a reasonable fraction of the maximum phonon energy, $E_{\mathrm{pn}, \max }$ in Table A.5, and so it is reasonable to conclude that acoustic-phonon generation is the primary energy-loss mechanism. Simple estimates for the positron thermalization times, done in Appendix $\mathrm{C}$, show that they are about the same or greater than the measured positron lifetimes. This means that the positrons may not be thermalized prior to annihilating and so a relatively low spur Ps formation fraction is expected. This could be true even if there were significant numbers of spur electrons inside the positron spur. In liquid and solid helium, and liquid neon there is the added complication that the spur electrons are known to self-trap into their own 'bubble' states within a few picoseconds. This means that thermalized positrons would have to find an electron in a bubble in order to form spur Ps.

Unlike positrons, Ps thermalization in the liquid rare gases is expected to be on the 
order of tens of picoseconds and the same or faster in molecular media. Khrapak (1995) has estimated that Ps will begin to trap at large density fluctuations, lose its excess kinetic energy (from the binding energy) in elastic collisions with the walls of the fluctuations and then be captured in a bubble state all within about $10^{-11}$ seconds in the liquid rare gases. These results are comparable to those of Tuomisaari et al. (1991) shown in Appendix C. These times are short compared to Ps lifetimes so it is expected that observable changes to the narrow peak intensity, due to spin-conversion collisions with spur electrons or impurities for example, must occur once the Ps is in the bubble state.

By contrast, positron spur Ps formation is likely to be significant in most molecular media. In these media, positrons generally have several additional thermalization mechanisms available below the last ionization energy. In most cases, the inelastic scattering threshold actually falls below the Ore gap. For example, in $\mathrm{N}_{2}$ low-energy positron collisions do not generate many triple state excitations due to the absence of exchange forces and the smallness of the spin-orbit coupling for a slow particle that is repelled from the nuclei. Gullikson and Mills (1990) have shown that the pcsitron inelastic threshold in solid $\mathrm{N}_{2}$ corresponds to the $\nu=0$ vibrational level of the $a^{\prime 1} \Sigma_{u}^{-}$band at $8.385 \pm 0.010 \mathrm{eV}$. Since this falls below the Ps formation threshold of $11.3 \mathrm{eV}$ it implies that many positrons can scatter out of the Ore gap relatively easily without forming Ps. Below the inelastic threshold, positrons in $\mathrm{N}_{2}$ on average lose about $30 \pm 5 \mathrm{meV}$ per collision (Gullikson and Mills 1990). This is consistent with optical phonon excitation being the primary energy-loss mechanism. This implies that positrons falling below the Ore gap will thermalize much quicker than in the condensed rare gases. The electron mobility in liquid $\mathrm{N}_{2}$ is low likely due to the formation of very temporary anion states (see (3.47)). The electrons therefore also thermalize quickly which means that the positron spur survives and that spur Ps formation in liquids is reasonable to expect.

\subsection{Positronium Bubble Model Results}

The bubble model discussed in $§ 3.4 .2$ is a simple theoretical model used to describe Ps in an equilibrium bubble. It is a good approximation at the liquid densities of interest here where the Ps binding energy to the bubble is large. Within this model, the bubble radius and potential-well depth uniquely determine the two measurable quantities from positron experiments - the narrow peak FWHM and the positron overlap probability with 
Table 6.4: Bubble radii estimated from calculation and experiment for simple liquids at their normal boiling point. The theoretical estimates were calculated by minimizing (3.35) with respect to the bubble radius. The surface tension parameters required for the calculations can be found in Tables A.3 and A.4. The potential well-depths for the finite potential-well model were measured from Figure 6.2.

\begin{tabular}{|c|c|c|c|c|c|}
\hline & \multicolumn{2}{|c|}{ Infinite potential-well model } & \multicolumn{3}{|c|}{ Finite potential-well model } \\
\hline & $\begin{array}{c}\text { From energy } \\
\text { minimization } \\
(\AA)\end{array}$ & $\begin{array}{c}\text { From measured } \\
\text { narrow peak } \\
(\AA)\end{array}$ & $\begin{array}{l}\text { Potential } \\
\text { well depth } \\
(\mathrm{eV})\end{array}$ & $\begin{array}{l}\text { From energy } \\
\text { minimization } \\
(\AA)\end{array}$ & $\begin{array}{c}\text { From measurec } \\
\text { narrow peak } \\
(\AA)\end{array}$ \\
\hline $\mathrm{He}$ & 18.8 & $21.0 \pm 1.3$ & 0.76 & 17.7 & $18.8 \pm 1.3$ \\
\hline $\mathrm{H}_{2}$ & 10.5 & $13 \pm 2$ & & & \\
\hline $\mathrm{Ne}$ & 8.4 & $8.9 \pm 0.3$ & 3.1 & 7.8 & $7.9 \pm 0.3$ \\
\hline \multirow[t]{2}{*}{$\mathrm{N}_{2}$} & 7.2 & $7.7 \pm 0.7$ & & & \\
\hline & & $8.7 \pm 0.7$ & & & \\
\hline Ar & 6.6 & $7.9 \pm 0.7$ & 1.6 & 5.8 & $6.0 \pm 0.6$ \\
\hline $\mathrm{Kr}$ & 6.3 & 6.4 & & & \\
\hline $\mathrm{Xe}$ & 6.1 & 5.9 & & & \\
\hline
\end{tabular}

the sample from lifetime experiments, or vice versa. As a first approximation an infinite potential-well depth was assumed. A theoretical estimate of the bubble radius was obtained using (3.36). Equation (3.43) and the FWHM of the narrow component shown in Table 6.2 were used to get an experimental estimate of the bubble radius. The results are shown in Table 6.4. The results from other authors for liquid $\mathrm{Kr}, \mathrm{Xe}$ and $\mathrm{H}_{2}$ have been included in the table for completeness. In view of the approximations involved and the uncertainties in some of the parameters, the agreement between the experimentally deduced bubble sizes and the predicted values is quite good.

The more realistic finite potential well model has also been used to analyze the data for liquid helium, neon and argon. Figure 6.3 is a semi-log plot of the equilibrium bubble radius as function of the depth of the spherical square-well potential. Each curve begins at the point where the square-well model predicts the Ps first binds to the bubble - i.e. where the bubble energy calculated from (3.35) is equal to the well depth and the Ps binding energy to the bubble is zero. These well depths correspond to significantly higher temperature, lower density fluids where a transition region exists in which both delocalized and localized bubble states are populated. The simple bubble model cannot describe this transition region. The more sophisticated models used to describe the onset of Ps bubble formation are mentioned in \$3.4.2. As the well depth in Figure 6.3 increases beyond the starting point 


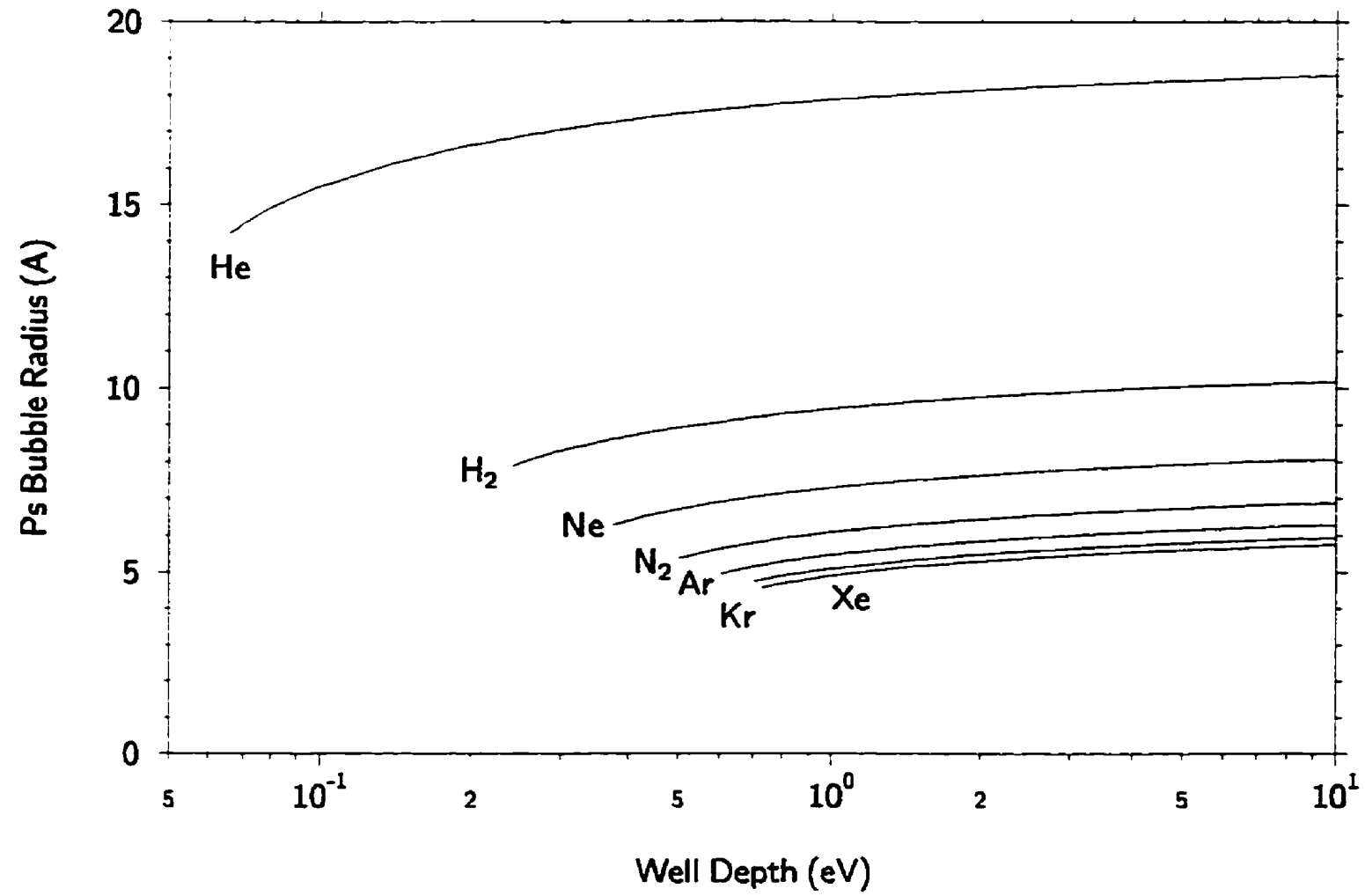

Figure 6.3: A semi-log plot of the Ps bubble radius as a function of the square-well potential depth for simple liquids. The starting point for each curve is where the bubble energy is equal to the well depth - i.e. the Ps binding energy to the bubble is zero. Beyond this starting point, the Ps binding energy to the bubble increases as the well depth increases. 


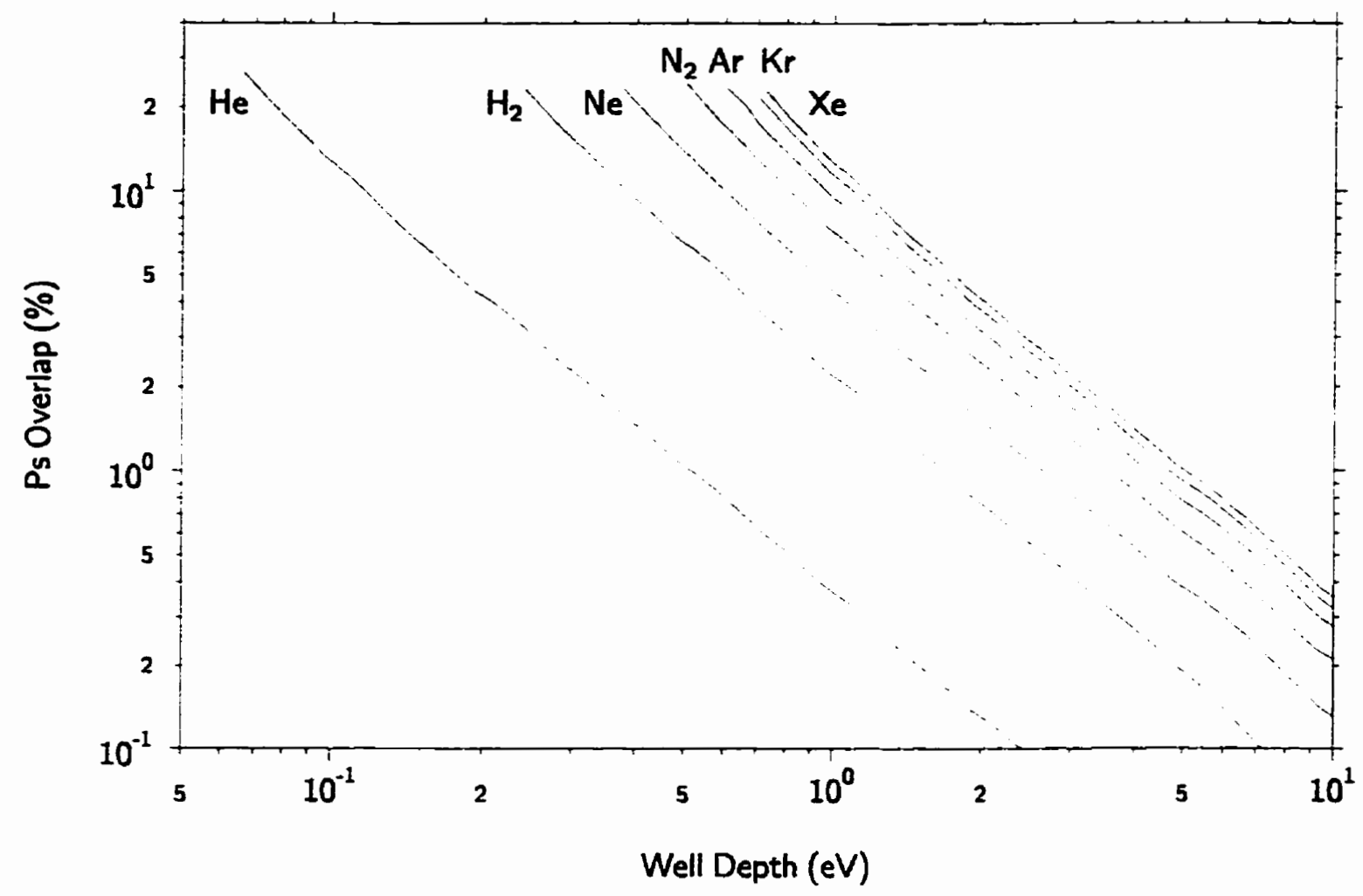

Figure 6.4: A log-log plot of the overlap of Ps in a bubble, as defined by (3.38), for several simple liquids at their normal boiling point.

both the equilibrium bubble radius and energy show only a weak dependence on the well depth. This implies that Ps is becoming more strongly bound to the bubble as the well depth increases and that the bubble state becomes more strongly favoured thermodynamically over the delocalized state. Table 6.4 shows the finite well depths corresponding to the delocalized Ps energies calculated for helium, neon and argon in $\S 6.1$. Notice that the finite well model predicts equilibrium bubble radii that are about $1 \AA$ smaller than the infinite well model predictions. The radii deduced from the experimental FWHM are also slightly smaller than those deduced using the infinite well model and result in a better agreement between theory and experiment. All of the results in Table 6.4 and Figure 6.3 assumed a zero Ps scattering length. Inserting the scattering lengths from Table 6.1 into the calculations increases the theoretically predicted bubble radius by about two-thirds of the scattering length (i.e. 0.5 to $0.6 \AA$ for helium, neon and argon). The experimental estimates of the bubble radius are also increased by an amount equal to the scattering length.

Figure 6.4 shows a $\log -\log$ plot of the overlap between $P s$ in a bubble and the medium, calculated using (3.38), as a function of the potential-well depth for several liquids. In 
contrast to the bubble radius and energy, the calculated Ps overlap has a strong dependence on the well depth. For an infinite well depth, the predicted overlap probability and pick-off annihilation rate are obviously zero. Even at finite well depths shown in Table 6.4 the predicted overlap probability is still about an order of magnitude smaller than that predicted by lifetime experiments. For example, in liquid helium Hautojärvi et al. (1976) had to use a shallower well depth of $0.25 \mathrm{eV}$ and a smaller bubble radius of $13.8 \AA$ in order to obtain the Ps wave function overlap needed to fit their measured $o$-Ps lifetimes. The reasons for this discrepancy are not fully resolved. It arises in part from the assumption that the potential is a square well with a discontinuous density change at the bubble surface. A more realistic smooth and continuous density profile would increase the overlap, and therefore the pickoff annihilation rate, while having little effect on the other bubble parameters which are reasonably insensitive to the exact shape of the potential. However, the density functional calculations of Nieminen et al. (1980) suggest that the density profile for Ps bubble in liquid helium at the densities considered here is actually well represented by a step function.

The discrepancy could also result from the fact that in the bubble model the Ps is treated as a point particle with no account being taken for its finite extent or its polarizability. The model simply predicts the overlap of the centre-of-mass of the Ps atom with the medium. However, this is not correct for lifetime measurements since they actually measure the positron overlap with the medium. Ps is a very disturbable atom - its polarizability being 8 times that of hydrogen. This implies that the overlap of the positron in Ps with the medium is greater than the centre-of-mass calculation would suggest. An overlap calculation including Ps polarization effects is expected to yield more consistent results. Chen and Stott (1992) have started preliminary calculations. The main result from their work is that the distribution for the electron in Ps in a bubble is more localized that the distribution for the positron, as expected.

Figure 6.5 shows a representative sample of narrow momentum components measured in helium, neon, argon and $\mathrm{N}_{2}$ under a variety of conditions. Figure 6.5a shows narrow components for liquid helium with several different magnetic fields applied. The dashed lines are the momentum distributions predicted using the infinite square well model (3.42). The solid lines are the prediction of the finite potential-well model calculated using (3.45) and the well depths in Table 6.4. For each narrow peak, the theoretical prediction has been arbitrarily normalized to give the best fit to the data. Notice that the infinite potential-well model always predicts a slightly wider, less peaked narrow component. The 

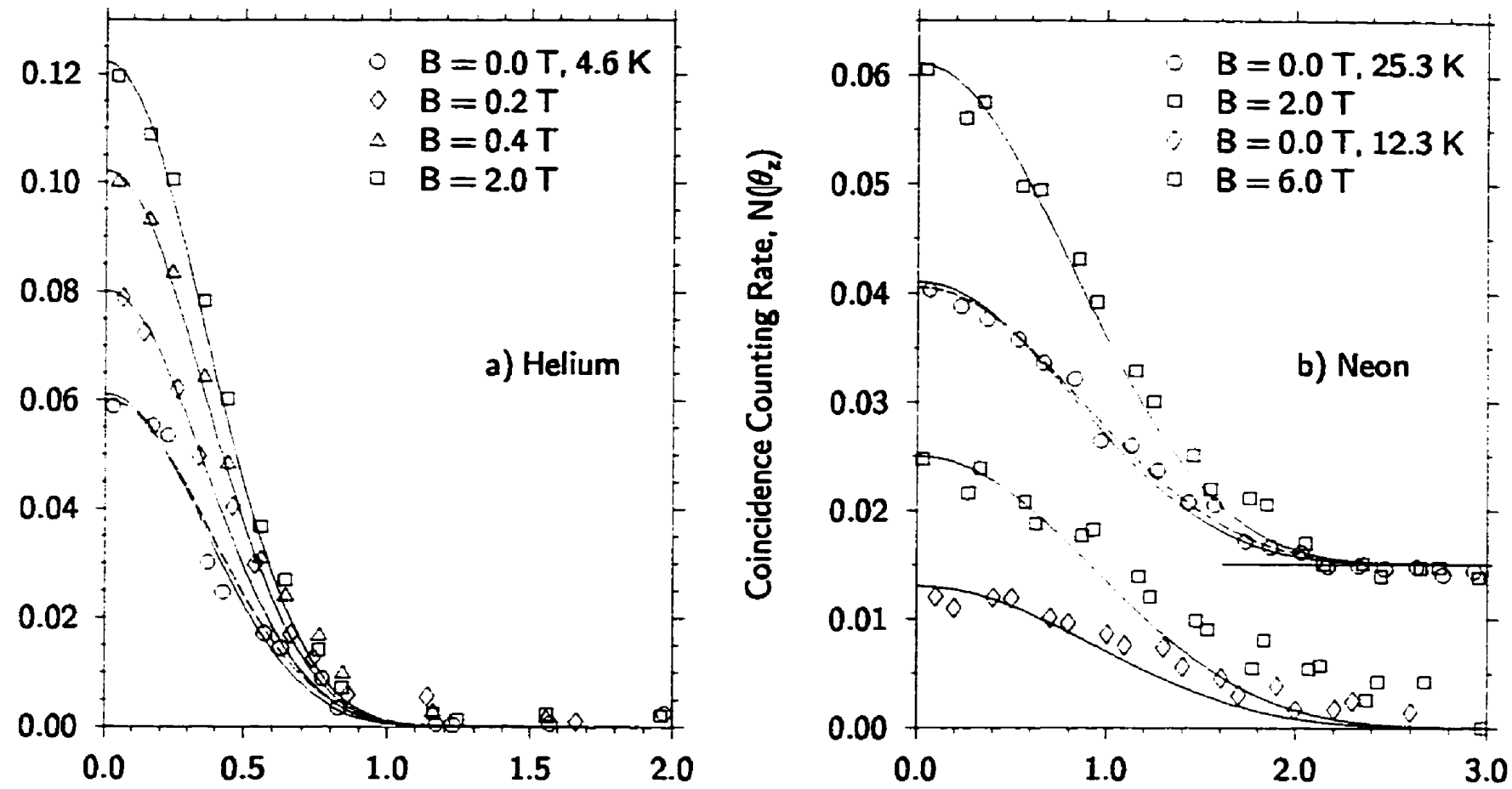

Angle Between Annihilation Photons, $\theta_{\mathbf{z}}$ (mrads)
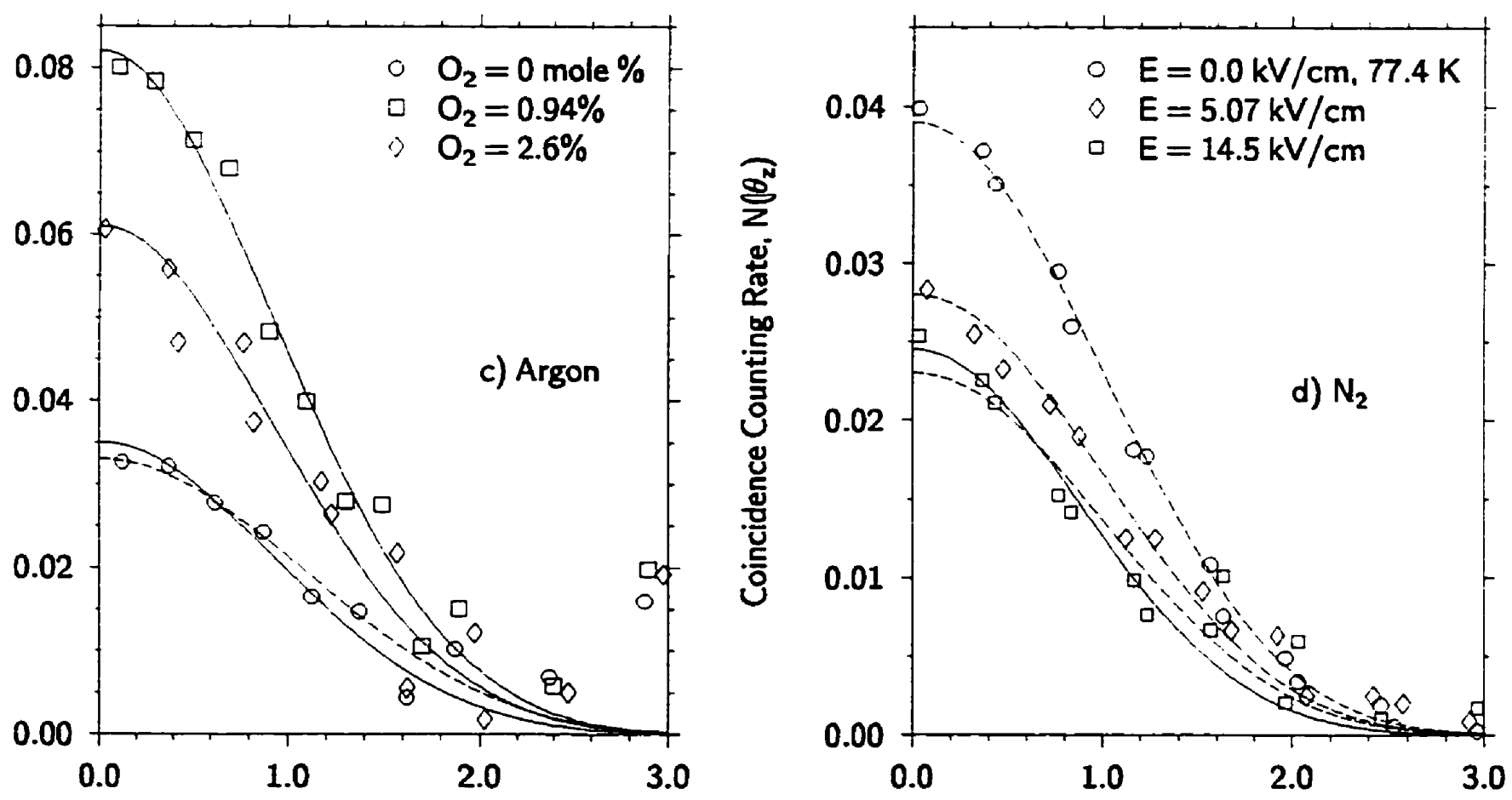

Figure 6.5: The narrow momentum component in a) liquid helium, b) liquid and solid neon, c) liquid argon, and d) liquid $\mathrm{N}_{2}$ obtained by subtracting the Gaussian fit to the broad component described in Table 4.2 from the total 1D-ACAR spectra. Data has been plotted for a variety of conditions - with and without magnetic and electric fields applied and with $\mathrm{O}_{2}$ impurity - to show that the bubble model remains a reasonable description in all of these conditions. 
upper two data sets in Figure 6.5b are narrow components in liquid neon. The lower two data sets are representative narrow peaks in solid neon. For solid neon a well depth of 6 $\mathrm{eV}$ was used in the finite potential-well model calculation. The specific surface energy in the solid, $\sigma_{s}$, was also scaled using a result quoted by Cohen and Jortner (1969)

$$
\sigma_{s}=\sigma_{l} \frac{\rho_{s}^{2}}{\rho_{l}^{2}}
$$

where $\sigma_{l}$ is the liquid surface tension and $\rho_{s}$ and $\rho_{l}$ are the solid and liquid densities, respectively. The increases to the well depth and the surface energy both had the effect of increasing the width of the predicted narrow component. Figure $6.5 \mathrm{c}$ shows narrow components for liquid argon at various $\mathrm{O}_{2}$ impurity levels, while Figure 6.5d shows similar results for liquid $\mathrm{N}_{2}$ with various static electric fields applied.

In general, the narrow peaks predicted by both the infinite and the finite potentialwell models are in good agreement with the experimental results for the liquids except, perhaps, at large angles. This is not very serious for several reasons. First, the accuracy of the data at large angles is low since as the narrow component approaches zero it depends critically on the choice of broad component. Second, a narrow component in excess of theory at larger angles implies more p-Ps annihilations occurring at higher momenta than predicted by the equilibrium bubble model. These higher momentum annihilations are to be expected, and are likely the result of: annihilations that occur before the bubble is fully expanded; residual atoms left inside the bubble; and Ps annihilations from excited states of the bubble system.

The narrow peak results in solid neon and argon are not described as well by the bubble model. This is again consistent with the idea that the Ps is not annihilating from self-trapped bubble states but from pre-existing defects. In contrast to the liquids, there was a large variation in the measured narrow peak FWHM for different solid samples. The narrow peak FWHM in solid neon suggests that the Ps is annihilating from voids that range from about 6 to $8 \AA$ in radius. In solid argon the voids ranged from about 5 to $10 \AA$ in radius. 


\subsection{Magnetic Field Effects}

Some of the most interesting results in this thesis were obtained from the magneticquenching 1D-ACAR experiments in condensed helium, neon, argon and $\mathrm{N}_{2}$. Figures 4.3 , 4.5 and 4.6 show the results of these experiments for liquid helium, liquid neon and solid neon, respectively. All of these narrow components are well described by the bubble model. Figure 4.4 plots the narrow peak intensities as a function of the magnetic field intensity. Also shown are the theoretical predictions of (3.56) which gives a virtually identical result to the Mills (1975) expression (3.60). For these calculations, the internal contact density $\kappa$ was set to 1 which should be a reasonable first approximation for Ps in a bubble. The nature of the experimental apparatus, with a positron source both above and below the sample, implies a net positron spin polarization of zero (i.e. $P=0$ ). For liquid helium, experimental values were used for the required annihilation rates in (3.56). Despite the limited number of data points the fit between the data and theory is very good. This implies that there are no other quenching mechanisms present in liquid helium apart from the magnetic field.

There exists no lifetime data for liquid neon. The pick-off annihilation rate was therefore varied to get the best fit to the narrow peak intensity data. Figure 4.4 shows that an $o$-Ps lifetime, $\tau_{3}$, of between $4.8 \mathrm{~ns}$ and $6.5 \mathrm{~ns}$ in (3.56) would give a reasonable fit to the data. These estimates are consistent with those for the other rare-gas liquids. If the fitted lifetime value is reasonable then the magnetic field is once again the only quenching mechanism present. Figure 4.4 also shows a sparse set of data for a single solid neon sample. While the narrow peak intensities in solid $\mathrm{Ne}$ and $\mathrm{Ar}$ were very inconsistent between different samples the change in $I_{n}$ as a function of magnetic field behaved very well for any given sample. Once again the data is fit quite well using the experimental long-lived o-Ps lifetime of $4.8 \mathrm{~ns}$. This result quite nicely confirms that Ps was formed in solid Ne.

Figures 4.7 and 4.8 show the results of the magnetic-quenching experiments for liquid and solid Ar. Figure 4.9 shows quite clearly that unlike $\mathrm{He}$ and $\mathrm{Ne}$ the narrow peak intensities for liquid argon fall well short of what is predicted by (3.56) when the experimental lifetimes are used. This is consistent with the fact that the zero-field narrow peak intensity for liquid argon is much larger than one third of the long-lived intensity measured by lifetime experiments. Both results imply that some other quenching and/or spin conversion mechanism must be at work in liquid argon. Reasonable possibilities would 
be Ps spin-conversion collisions with impurities having unpaired electrons, such as $\mathrm{O}_{2}$, or with various spur species, such as secondary electrons, interacting with the Ps at the bubble surface. Once again, this problem cannot be an artifact of the broad component subtraction. The broad component used in argon was basically the 1D-ACAR curve in solid Ar which represents the upper limit on the broad component. A broad component that was lower in the low momentum region is possible but it would only increase the zero-field narrow peak intensity and make the relative increase in $I_{n}$ as a function of magnetic field even smaller.

The magnetic-quenching results shown in Figure 4.12 for liquid $\mathrm{N}_{2}$ are again ambiguous. Using the best fit Gaussian to describe the broad component gives a zero-field narrow peak intensity that is about $8.6 \%$ and rises to $13.4 \%$ at high magnetic fields in Figure $4.13 \mathrm{a}$. As in liquid argon, this rise is significantly lower than the doubling expected if the magnetic field was the only quenching mechanism present. Figure $4.13 \mathrm{~b}$ plots the same thing, however this time the dashed line in Figure 4.12 was used as the broad component. This estimate reduces the narrow peak intensity by $3.5 \%$ and results in a theoretical prediction that corresponds nicely with the data. While this second estimate for the broad component is completely arbitrary it does demonstrate the importance of having a reasonable estimate for the broad component. The proper interpretation of the liquid $\mathrm{N}_{2}$ magnetic field results are in doubt - it is unclear if there are other quenching mechanisms present or not.

\subsection{Electric-Field Effects}

Figures 4.7 and 4.15 show the ID-ACAR results for liquid argon and $\mathrm{N}_{2}$ with various applied electric field strengths. In Figure 4.16 the narrow peak intensity is plotted as a function of the static electric field strength. The expected effect of the electric field was to sweep both secondary electrons and the positron out of the positron spur and away from each other. Increasing electric field should therefore decrease spur Ps formation as well as reduce the possibility of Ps spin-conversion with spur electrons.

In liquid argon there is a small decrease in $I_{n}$ with electric field strength, that may or may not be significant given the size of the error estimates. Given that appreciable spur Ps formation is unlikely in liquid argon, the reduction of about $1 \%$ in $I_{n}$ is most probably due to a reduction in the interactions with spur electrons. 
In Figure 4.16a the liquid $\mathrm{N}_{2}$ narrow peak intensity was calculated using the best fit Gaussian broad component. For liquid $\mathrm{N}_{2}$ there is a significant decrease in $I_{\mathrm{n}}$ with increasing field. Also shown are three data points for liquid $\mathrm{N}_{2}$ in a high magnetic field. Figure $4.16 \mathrm{~b}$ replots the same results this time using the more peaked broad component estimate. It also shows the narrow peak intensities implied by the lifetime measurements of Pepe et al.(1993) assuming the that the p-Ps:o-Ps ratio of 1:3 (i.e. their $I_{3}$ results were simply divided by 3). The correspondence between the two data sets is remarkable. These results and those of lifetime experiments (Pepe et al. 1993) show that the Ps intensity decreases in liquid $\mathrm{N}_{2}$ with increasing electric field. This would be expected if spur Ps formation was significant.

\subsection{Effects of $\mathrm{O}_{2}$ Impurity}

To study the effects of $\mathrm{O}_{2}$ impurity on the Ps yield, a series of experiments were performed in which small quantities of $\mathrm{O}_{2}$ were deliberately added to the liquid $\mathrm{Ar}$ and $\mathrm{N}_{2}$ samples. As with the magnetic and electric fields experiments the addition of $\mathrm{O}_{2}$ had no measurable effect on the shape of the broad component or on the FWHM of the narrow peak. It did, however, have a dramatic effect on the narrow peak intensities as can be seen in Figures 4.10, 4.11 and 4.14.

In both $\mathrm{Ar}$ and $\mathrm{N}_{2}$, the narrow peak intensity increases rapidly for small $\mathrm{O}_{2}$ concentrations, reaches a maximum at about 0.5 mole\% and then has a linear decreasing behaviour. The main effects of the $\mathrm{O}_{2}$ impurity are to change the positron and electron thermalization rates and to cause $\mathrm{Ps}$ spin conversions. In argon, low $\mathrm{O}_{2}$ concentrations should not have much effect on the amount of Ore gap Ps formed. Positrons falling in energy between the lower Ore gap boundary and the inelastic threshold will still remain hung up in the Ore gap. Ionizing collisions with $\mathrm{O}_{2}$ may also add to the Ore gap Ps formation (i.e. the $\mathrm{O}_{2}$ creates a second Ore gap), though this is not expected to be significant. As the $\mathrm{O}_{2}$ concentration increases, the Ore gap Ps formation will decrease somewhat as positrons are able to thermalize through the Ore gap faster since $\mathrm{O}_{2}$ has inelastic modes that are well below the Ore gap. For positrons that do fall below the main Ore gap, the main effect of the $\mathrm{O}_{2}$ is to allow the positron to thermalize more rapidly. Furthermore, as the $\mathrm{O}_{2}$ concentration increases, spur electrons also thermalize more quickly and both the free ion yield and the size of the spur is reduced, as shown in Table 3.1. The implication is that spur Ps can now readily form in liquid $\mathrm{Ar}$, which would account for some of the dramatic increase in the 
narrow peak intensity seen in Figure 4.11. The fast positron thermalization time in liquid $\mathrm{N}_{2}$ implies that the increase in its narrow peak intensity is due mostly to an increase in the Ps spin conversion rate. However, some of the increase will be due to an increase in the Ps formation fraction.

The increase in Ps yield is confirmed by the lifetime experiments of Spektor and Paul (1971) in liquid $\mathrm{Ar}$ and $\mathrm{N}_{2}$. Their results also show an increase in $I_{3}$ for low $\mathrm{O}_{2}$ impurity concentrations (i.e. the increase seen in the 1D-ACAR experiments is not simply o-Ps $\rightarrow$ p-Ps conversion). Here, $I_{3}$ in argon increased from $7.0 \%$ in pure argon to $14.6 \%$ at 8.0 torr $\mathrm{O}_{2}$. $I_{3}$ only increased from $13.9 \%$ in pure liquid $\mathrm{N}_{2}$ to $14.3 \%$ at 5.0 torr of $\mathrm{O}_{2}$ and was approaching a limit at higher $\mathrm{O}_{2}$ concentrations. Increasing $I_{n}$ and $I_{3}$ implies increasing Ps formation fractions. However, some of the increase in the narrow peak intensities is also due to elastic and inelastic Ps spin-conversions with the $\mathrm{O}_{2}$.

The maximum and then gradual decrease in $I_{n}$ versus $\mathrm{O}_{2}$ is due, in part, to the reduction in free ion yield that occurs with increasing $\mathrm{O}_{2}$ concentration. That is, as $\mathrm{O}_{2}$ concentration increases electrons begin to thermalize closer and closer to their parent ions such that fewer spur electrons escape recombination. Increasing $\mathrm{O}_{2}$ concentration effectively collapses the positron spur thereby reducing the spur Ps formation. This may also help explain the $1 \mathrm{D}$-ACAR results observed in pure liquid $\mathrm{O}_{2}$. The curve seems to consist simply of a broad component with no narrow peak (Stewart et al. 1990). The most reasonable explanation is that the low-energy excitations in $\mathrm{O}_{2}$ allow both the positrons and the electrons to thermalize extremely fast. The positrons slow down so fast that they pass right through the Ore gap forming little or no Ps (i.e. $\gamma_{\mathrm{r}} \gg \gamma_{\mathrm{Ps}}$ in (3.18)). The electrons slow down so quickly that they do not escape the Coulomb field of their ion. This is confirmed in Table 3.1 which shows a free ion yield and an average initial electron-ion separation that decreases significantly with increasing $\mathrm{O}_{2}$ concentration. In pure $\mathrm{O}_{2}$ the free ion yield is two orders of magnitude lower than in pure liquid Ar. This implies that the spur electrons thermalize and recombine so quickly in $\mathrm{O}_{2}$ that there are simply none left for spur Ps formation.

\subsection{Spin-Exchange Model Calculations}

The results of the previous sections show that some kind of Ps quenching or spinconversion mechanism is occurring in liquid argon. It is expected that this is also true for 
liquid krypton and xenon. The ambiguity in the choice of broad component makes it unclear if it is true for liquid $\mathrm{N}_{2}$ as well. The most likely quenching mechanisms are spin-exchange collisions with either $\mathrm{O}_{2}$ impurity or with excess spur electrons. In what follows, the model developed in $\$ 3.5 .3$ to described spin-exchange collisions in a magnetic field will be applied to the liquid argon results.

The results of $\S 6.6$ have already shown that small amounts of $\mathrm{O}_{2}$ impurity can have a dramatic effect on the narrow peak intensity. Despite the experimental precautions to avoid $\mathrm{O}_{2}$ impurity, it is possible that small amounts of $\mathrm{O}_{2}$ were present in the system. $\mathrm{O}_{2}$ and other impurities would have little effect on the helium and neon samples as they are likely to have frozen out onto the walls of the $1.3 \mathrm{~m}$ long support tubing leading to the sample chamber. Since the $\mathrm{O}_{2}$, or any other impurity, concentration is constant over time the spin-exchange conversion rates in (3.71) and (3.72) become time independent and proportional to (3.67). Numerical analysis shows that the field dependence of the $\gamma_{i j}$ is weak and has virtually no effect on the calculations other than to complicate them. If the field dependence is also ignored then the conversion rates in (3.72) reduce to a single time and field-independent constant, $\gamma$. Finally, if it is assumed that the $p$-Ps annihilations are fast compared to the spin-conversion rate (i.e. $\left.\lambda_{1}^{o} \gg \gamma\right)$ then $(3.71)$ reduce to

$$
\begin{aligned}
& \frac{d n_{1}(t)}{d t}=-\lambda_{1} n_{1}+\gamma\left(n_{2}+n_{3}+n_{4}\right) \\
& \frac{d n_{2}(t)}{d t}=-\left(\lambda_{3}+2 \gamma\right) n_{2}+\gamma n_{3} \\
& \frac{d n_{3}(t)}{d t}=\gamma n_{2}-\left(\lambda_{3}+3 \gamma\right) n_{3}+\gamma n_{4} \\
& \frac{d n_{4}(t)}{d t}=\gamma n_{3}-\left(\lambda_{3}+2 \gamma\right) n_{4}
\end{aligned}
$$

and can be solved analytically to give

$$
\begin{aligned}
& n_{1}(t)=\frac{1}{4}\left(\frac{-\lambda_{1}+\lambda_{3}+4 \gamma}{-\lambda_{1}+\lambda_{3}+\gamma}\right) e^{-\lambda_{1} t}-\frac{3}{4}\left(\frac{\gamma}{-\lambda_{1}+\lambda_{3}+\gamma}\right) e^{-\left(\lambda_{3}+\gamma\right) t} \\
& n_{2}(t)=-\frac{P}{4} e^{-\left(\lambda_{3}+2 \gamma\right) t}+\frac{1}{4} e^{-\left(\lambda_{3}+\gamma\right) t} \\
& n_{3}(t)=\frac{1}{4} e^{-\left(\lambda_{3}+\gamma\right) t} \\
& n_{4}(t)=\frac{P}{4} e^{-\left(\lambda_{3}+2 \gamma\right) t}+\frac{1}{4} e^{-\left(\lambda_{3}+\gamma\right) t}
\end{aligned}
$$

These equations are the same as those derived by Bisi et al. (1981) except that the effects of positron polarization $P$ are also included here. The narrow peak intensity is now easily estimated by inserting these results into (3.56). 


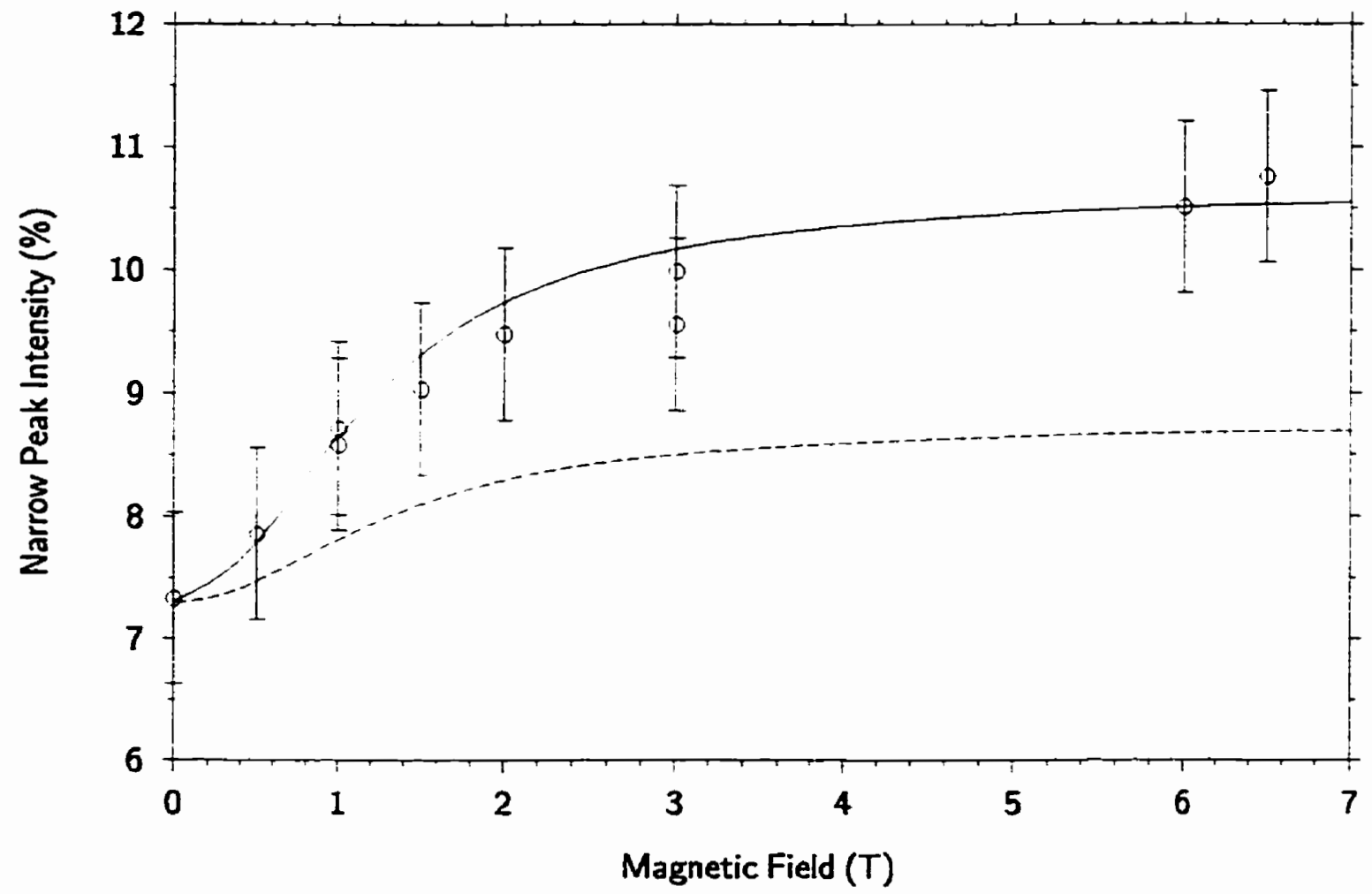

Figure 6.6: This plot shows the intensity of the narrow peak as a function of magnetic field in liquid argon. The dashed line corresponds to the narrow peak intensity predicted by inserting (6.9) into (3.56) and using a pick-off annihilation rate and spin-conversion rate predicted by $(6.10)$ and $(6.11)\left(\lambda_{\text {po }}=0.45 \times 10^{8} / \mathrm{s}, \gamma=1.02 \times 10^{8} / \mathrm{s}\right)$. The solid line corresponds to the choice of $\lambda_{\mathrm{po}}=0.92 \times 10^{8} / \mathrm{s}$ and $\gamma=0.55 \times 10^{8} / \mathrm{s}$.

In zero magnetic field the longest-lived component, $\tau_{3}$, in the lifetime spectrum has a decay rate

$$
\frac{1}{T_{3}}=\lambda_{3}^{o}+\gamma
$$

Similarly, it is straightforward to show that to first order the ratio of the zero-field narrow peak intensity to the long lifetime component intensity is

$$
\frac{I_{\mathrm{n}}(0)}{I_{3}(0)}=\frac{1}{3} \frac{\lambda_{3}^{\circ}+4 \gamma}{\lambda_{\mathrm{po}}+\gamma}
$$

where the positron polarization was again set to zero. Inserting the experimental values for liquid argon $\left(\tau_{3}=6.5 \mathrm{~ns}, I_{n}(0)=7.3 \%\right.$, and $\left.I_{3}=7.0 \%\right)$ into (6.10) and (6.11) gives a pick-off annihilation rate of $\lambda_{\mathrm{po}}=0.45 \times 10^{8} / \mathrm{s}$ and a spin-conversion rate of $\gamma=1.02 \times 10^{8} / \mathrm{s}$. These results have little dependence on the internal contact density, $\kappa$, so it was again set to 1 for Ps in a bubble. Inserting (6.9) and these estimates into (3.56) gives the dashed line 
shown in Figure 6.6. Obviously (6.11) over estimates the spin-conversion rate and results in an $o$-Ps quenching that is far too large. The solid line in Figure 6.6 is simply an arbitrary fit to the data obtained by varying $\lambda_{\text {po }}$ and $\gamma$ subject to (6.10). The best fit was achieved using $\lambda_{\mathrm{po}}=(0.92 \pm 0.05) \times 10^{8} / \mathrm{s}$ and $\gamma=(0.55 \pm 0.05) \times 10^{8} / \mathrm{s}$. At this conversion rate about $50 \%$ of the Ps self-annihilates as $p$-Ps which implies a total Ps fraction of just under $15 \%$. 


\section{Chapter 7}

\section{Conclusions}

Angular correlation experiments were performed in several simple condensed insulating media in order to study the effects of various physical and chemical processes on Ps yields. In order to accomplish this, it was necessary to have a reasonable separation of the broad positron momentum component from the more narrow p-Ps component. Previous gas scattering calculations give a first approximation to the broad component but these predictions are not adequate. Most studies in condensed matter have not worried about the annihilations with core electrons. Those that have studied the materials of interest to this thesis do not provide satisfactory fits to the experimental data either. The possible existence of an intermediate momentum component due to some other annihilation process is unlikely. Therefore an attempt was made to calculate improved positron momentum distributions and lifetime predictions for the heavier condensed rare gases. To start this calculation realistic positron-rare gas potentials were generated. The potential$s$ that included the Abarenkov polarization potential generated positron band structures that best fit the available experimental data. These potentials were then used to calculate positron wave functions using the pseudopotential method. Ignoring the positron-core electron correlations these wave functions were used to calculate IPM approximations for the broad momentum component and the positron lifetimes. As expected, these results did not provide an adequate theoretical description. To improve the calculations, a semiphenomenological form of the Jarlborg and Singh enhancement scheme was used to include the positron-core electron correlation effects. These results, for the first time, provided a reasonable description of the broad components in the condensed rare gases and very good predictions for the positron lifetimes.

The angular-correlation measurements in liquid helium, neon, argon and $\mathrm{N}_{2}$ with no magnetic field applied all show the narrow momentum component due to the selfannihilation of $p$-Ps from localized bubble states. A comparison of the free Ps energy to the energy of the bubble state shows that the Ps bubble is strongly favoured thermo-

dynamically. The shape of the experimental narrow component is described quite well by the spherical square-well bubble model in all cases. The model also predicts the bubble 
radii deduced from the FWHM of the narrow peak with good accuracy. However, it was suggested that the usual bubble model does not correctly predict the Ps overlap with the medium needed for calculations of the Ps pick-off annihilation rate. Instead a more sophisticated model is needed that includes the effects of Ps polarization so that the overlap of the positron in Ps is calculated instead of the Ps centre-of-mass overlap. Such a model might be able to simultaneously describe angular correlation and positron lifetime results.

Angular-correlation experiments with applied magnetic fields in condensed helium, neon, argon and $\mathrm{N}_{2}$ were done for the first time. In liquid helium the increases in the narrow peak due to the mixing of Ps states followed theoretical predictions. The lack of a positron lifetime measurement in liquid neon makes it impossible to predict the narrow peak increase as a function of magnetic field. However, a fit to the experimental results suggest a long-lived o-Ps lifetime of between 4.8 and $6.5 \mathrm{~ns}$.

By contrast, the magnetic field effect in liquid argon was surprisingly small. It was postulated that another quenching mechanism, spin-exchange conversions between $p$-Ps and $o$-Ps due to either free electrons in the positron spur or due to $\mathrm{O}_{2}$ impurity, was competing with the magnetic field. A theoretical model describing the behaviour of Ps in a magnetic field that includes the effects of time-dependent spin-exchange collisions was developed. After several simplifying assumptions, including making the spin-exchange conversion rates constant in time, the model reduces to the one generally used in positron physics. This simplified model, which is appropriate for describing the effects of $\mathrm{O}_{2}$ impurity with a constant concentration, predicts a spin-conversion rate of $\gamma=(0.55 \pm 0.05) \times 10^{8} / \mathrm{s}$. An application of the more sophisticated model, which would be applicable to Ps spin-exchange conversions on spur electrons whose density decreases exponentially with time, has yet to be completed.

The free Ps energy calculations suggest that free Ps is unlikely in solid neon and argon since the free energy is greater than the binding energy. However, the magnetic field experiments confirm that Ps does exist in these solids. It is reasonable to expect that the Ps either quickly traps at a defect, which it would see as a potential well, or actually forms inside of one. This is consistent with the large variation in the narrow peak intensities observed between samples of solid neon and argon. The variation is the result of the relatively uncontrolled crystal growing conditions resulting in solids with various defect concentrations and sizes. 
The electric field and $\mathrm{O}_{2}$ impurity experiments in liquid argon and $\mathrm{N}_{2}$ provide useful insight into the Ps formation process and help to resolve the controversy over whether the Ore gap or the spur formation model is correct in condensed media. In the condensed rare gases it is expected that the Ore gap is the predominate Ps formation mechanism. This is expected to be the case for any large band-gap insulator where the positron thermalization is slow. In these materials, positrons that touch-down inside the Ore gap during their thermalization are likely to be hung up there until they form Ps. Positrons that fall below the Ore gap may simply thermalize too slowly to form significant Ps with spur electrons. The spur formation model is expected to become important in condensed media where the positron thermalization is faster, such as in liquid $\mathrm{N}_{2}$. This is especially true if the inelastic scattering threshold falls below the lower Ore gap boundary. In this case, positrons can quickly scatter below the Ore gap and little Ore gap Ps is formed. Once below the Ore gap the positrons can quickly thermalize and some fraction will form Ps with spur electrons. This simple qualitative picture is consistent with the electric fields results. The electric field will, in part, sweep the free electrons out of the positron spur making it more difficult to form spur Ps. Liquid $\mathrm{N}_{2}$ showed a significant decrease in Ps with electric field. The effect in liquid argon was much smaller. The electric field should also decrease the possibility of Ps spin-exchange collisions. The small decrease in Ps yield with electric field observed in liquid argon suggests that either spin-exchange collisions with spur electrons is not significant or that the electric field was not successful in separating the Ps and electrons.

The $\mathrm{O}_{2}$ impurity results also confirm the idea that the relative importance of the two Ps formation models is based largely on the positron thermalization time. The $\mathrm{O}_{2}$ has two main effects: it increases both the positron and electron thermalization rates; and allows Ps spin-exchange collisions. For both liquid argon and $\mathrm{N}_{2}$, small concentrations of $\mathrm{O}_{2}$ increase both $I_{n}$ and $I_{3}$ which implies an increasing Ps yield. Since $\mathrm{O}_{2}$ helps the positrons to scatter out of the Ore gap, the initial increase in $I_{n}$ must be due, in part, to increasing spur Ps formation. The maximum and gradual decrease in $I_{n}$ versus $\mathrm{O}_{2}$ concentration results from the $\mathrm{O}_{2}$ causing electrons to thermalize faster and closer to their parent ions so that fewer spur electrons escape recombination. The decreasing spur electron density results in a decrease in the spur Ps formation. 


\section{Bibliography}

[1] Abarenkov I.V. and Antonova I.M., 1974, Phys. Status Solidi B, 64, 747.

[2] Adkins, 1983, Ann. Phys. (N.Y.), 146, 78.

[3] Aguilar-Bentez M. et al., 1986, "Review of particle properties", Phys. Lett., 170B, 1.

[4] Ajzenberg-Selove F., 1990, "Energy levels of light nuclei A = 11-12", Nucl. Phys., A506, 1 158.

[5] Anderson C.D., 1932a, Science, 76, 238.

[6] Anderson C.D., 1932b, Phys. Rev., 41, 405.

[7] Anderson C.D., 1933, “The positive electron", Phys. Rev., 43, 491.

[8] Arponen J. and Pajanne E., 1979a, "Electron liquid in collective description. III. Positron annihilation", Ann. Phys. (N.Y.), 121, $343-389$.

[9] Arponen J. and Pajanne E., 1979b, “Angular correlation in positron annihilation", J. Phys. F, 9(12), $2359-2376$.

[10] Asai S., Orito S., Sanuki T., Yasuda M. and Yokoi T., 1991, "Direct search for orthopositronium decay into two photons", Phys. Rev. Lett., 66(10), 1298 - 1301.

[11] Ashcroft N.W. and Mermin N.D., 1976, Solid State Physics (Philadelphia: Saunders College).

[12] Barabash A.S., Golubev A.A. Kazachemko O.V. and Ovchinnikov B.M., 1982, "Attachment of low-energy electrons to $\mathrm{O}_{2}$ molecules in some gases and liquids: an instrument for measuring concentrations of electronegative impurities in gases", Sov. Phys. Tech. Phys., 27(10), 1261 1266.

[13] Barbielli B., Genoud P., and Jarlborg T., 1991, "Calculation of positron lifetimes in bulk materials", J. Phys.: Condens. Matter, 3, $7631-7640$.

[14] Barker M.I., and Bransden B.H., 1968, "The quenching of orthopositronium by helium", Proc. Phys. Soc., 1, 1109 - 1114.

[15] Beling C.D. and Smith F.A., 1980, "Possible improvements in the bubble model description of positronium pick-off annihilation in liquids", Chem. Phys., 49, 417-427.

[16] Bellotti E., Corti M., Fiorini E., Liguori C., Pullia A., Sarracino A., Sverzellati P., and Zanotti L., 1987, J. Phys. F, 17, 1477.

[17] Bereststskiī V.B., Lifshitz E.M. and Pitaevskii L.P., 1982, Quantum Electrodynamics (Toronto: Pergamon Press Ltd).

[18] Bergensen B. and Stott M.J., 1969, "Effect of vacancy formations on the temperature dependence of positron lifetime", Sol. St. Comm., 7, 1203.

[19] Bergensen B., Pajanne E., Kubica P., Stott M.J. and Stewart A.T., 1974, "Positron diffusion in metals", Sol. St. Comm., 15, $1377-1380$.

[20] Berko S. and Hereford F.L., 1956, "Experimental studies of positron interactions in solids and liquids", Rev. Mod. Phys., 28(3), 299 - 307.

[21] Berko S. and Plaskett J.S., 1958, "Correlation of annihilation radiation in oriented single metal crystals", Phys. Rev., 112(6), $1877-1887$. 
[22] Berko S., Haghgooie M. and Mader J.J., 1977, Phys. Lett. A, 63, 335.

[23] Berko S. and Pendleton H.N., 1980, "Positronium", Ann. Rev. Nucl. Part. Sci., 30, 543 - 581.

[24] Berko S., 1983, "Momentum density and Fermi-surface measurements in metals by positron annihilation" in [41], $64-145$.

[25] Bhat M.R., 1988, "Nuclear data sheets for $A=68^{*}$, Nucl. Data Sheets, 55, 1 - 70.

[26] Bisi A., Fiorentini A., Gatti E. and Zappa L., 1962, "Magnetic quenching of positronium in solids and positron helicity", Phys. Rev., 128(5), 2195 -2199.

[27] Bisi A., Consolati G., Gambarini G. and Zappa L., 1981, "Effects of collisions with paramagnetic ions on the magnetic quenching of positronium", il Nuovo Cimento B, 65(2), 442 453.

[28] Bisi A., Gambarini G. and Zappa L., 1983, "Electric-field effect on positronium formation in condensed matter", il Nuovo Cimento D, 2(5), 1465 - 1479.

[29] Bisi A., Consolati G., Gambarini G. and Zappa L., 1985, il Nuovo Cimento D, 6, 183.

[30] Bisi A., Consolati G., Quasso F. and Zappa L., 1987, "Exchange collisions between positronium and $\mathrm{Cu}^{2+}$ ions", il Nuovo Cimento $D, 9(6), 657-668$.

[31] Bonderup E., Andersen J.U., and Lowy D.N., 1979, "Enhancement of positron annihilation with core electrons", Phys. Rev. B, 20(3), $883-899$.

[32] Borghesani A.F. and Santini M., 1990, "Electron mobility and localization effects in highdensity Ne gas", Phys. Rev. A, 42(12), $7377-7388$.

[33] Boronski E. and Nieminen R.M., 1986, "Electron-positron density functional theory", Phys. Rev. $B, 34(6), 3820-3831$.

[34] Brandt W., Berko S. and Walker W.W., 1960, "Positronium decay in molecular substances", Phys. Rev., 120, 1289.

[35] Brandt W. and Spirn I., 1966, "Positron lifetime spectra in molecular substances", Phys. Rev.. $142,231$.

[36] Brandt W. and Feibus, 1968, "Electric field dependence of positronium formation in matter", Phys. Rev., 174(2), $454-459$.

[37] Brandt W., Coussot G. and Paulin R., 1969a, Phys. Rev. Lett., 23, 522.

[38] Brandt W. and Feibus, 1969b, "Subexcitation positrons", Phys. Rev., 184(2), 277 - 281.

[39] Brandt W. and Fahs J.W., 1970, "Positronium decay in condensed matter", Phys. Rev. B, 2, 1425.

[40] Brandt W. and Arista N., 1982, "Thermalization and diffusion of positrons in solids", Phys. Rev. $B, 26(8), 4229-4238$.

[41] Brandt W. and Dupasquier A. (eds.), 1983a, Positron Solid-State Physics (New York: NorthHolland Publishing Co.).

[42] Brandt W., 1983b, "Statistical dynamics of positrons in solids" in [41], $1-31$.

[43] Briscoe C.V. and Stewart A.T., 1967, "Positron annihilation in liquified gases" in [275], 377 -381 .

[44] Briscoe C.V., S.-I. Choi and Stewart A.T., 1968, "Zero-point bubbles in liquids", Phys. Rev. Lett., 20(10), 493 - 496. 
[45] Brusa R.S., Dupasquier A., Longano S. and Oss S., 1991, "Positron-electron annihilation in the proximity of a second electron in a dense medium", Phys. Rev. B, 43(6), $12715-12722$.

[46] Brusa R.S. and Dupasquier A., 1992, "Spin states of positron compounds" in [152], $197-203$.

[47] Bruschi L., Mazzi G. and Santini M., 1972, Phys. Rev. Lett., 28, 1504.

[48] Bruschi L., Santini M. and Torzo G., 1984, "Resonant electron attachment to oxygen molecules in dense helium gas", J. Phys. B, 17, $1137-1154$.

[49] Burrows T.W. and Bhat M.R., 1986, "Nuclear data sheets for $A=57^{* ”, ~ N u c l . ~ D a t a ~ S h e e t s, ~}$ 47, $1-135$.

[50] Byakov V.M., Gol'danskii V.I. and Shantarovich, 1974, Dokl. Akad. Nauk SSSR, 219, 633.

[51] Byakov V.M. and Petuchov V.R., 1983a, "Mechanism of positronium ortho-para- conversion in noble liquids", Radiochem. Radioanal. Lett., 58(2), 75 - 90.

[52] Byakov V.M. and Petuchov V.R., 1983b, "On the bubble model of positronium atom in liquids", Radiochem. Radioanal. Lett., 58(2), 91 - 102.

[53] Byakov V.M. and Grafutin V.I., 1986, "Positronium in radiation chemistry of liquids". Radiat. Phys. Chem., 28(1), 1 - 18.

[54] Campeanu R.I. and Humberston J.W., 1975, "The scattering of p-wave positrons by helium", J. Phys. B, 8(11), L244 - L248.

[55] Canter K.F. and Roellig L.O., 1970, "Critical behaviour of positrons in low-temperature gaseous helium", Phys. Rev. Lett., 25, $328-330$.

[56] Canter K.F., McNutt J.D. and Roellig L.O., 1975a, "Positron annihilation in low-temperature gases. I. helium", Phys. Rev. A, 12(2), $375-385$.

[57] Canter K.F. and Roellig L.O., 1975b, "Positron annihilation in low-temperature gases. II. argon and neon", Phys. Rev. A, 12(2), $386-395$.

[58] Carbotte J.P., 1966, "Theory of positron annihilation in real metals.", Phys. Rev., 144(1), $309-318$.

[59] Carbotte J.P. and Salvadori, 1967, "Positron annihilation in real metals. II. Calculation of core enhancement factors", Phys. Rev., 162(2), $290-300$.

[60] Chang Lee, 1958, "Stationary states of electron-positron systems and annihilation transitions", Sov. Phys. JETP, 6(33), $281-291$.

[61] Charlton M., Griffith T.C, Heyland G.R., Lines K.S. and Wright G.L., 1980, "The energy dependence of positronium formation in gases", J. Phys. B, 13, L757.

[62] Chariton M., Cullen R.A., Raza J. and Watts M.S.T., 1992, "Positronium formation in the heavier noble gases in the presence of a static electric field", J. Phys. B, 25, $4351-4361$.

[63] Chen J. and Stott M.J., 1992a, "Positronium polarization in liquids" in [152], 1513 - 1516.

[64] Chiba T., Dürr and Brandt W., 1977, "Enhancement effect on positron annihilation with tightly bound electrons", Phys. Status Solidi B, 81, $609-614$.

[65] Chow P.C., 1972, "Computer solutions to the Schrödinger equation", Am. J. Phys., 40, 730 $-734$.

[66] Chuang S.Y. and Tao S.J., 1974, "Quenching of positronium in nitrogen dioxide", Phys. Rev. A, 9(2), $989-992$. 
[67] Clementi E. and Roetti C., 1974, "Roothaan-Hartree-Fock atomic wavefunctions: Basis functions and their coefficients for ground and certain excited states of neutral and ionized atoms, $\mathrm{Z} \leq 54 "$, Atomic Data and Nucl. Data Tables, 14, 177 - 478.

[68] Cohen M.H. and Jortner J., 1969, "Electron cavity formation in solids helium", Phys. Rev., $180(1), 238-241$.

[69] Coleman P.G., Sharma S.C., and Diana L.M. (eds.), 1982, Proc. $6^{\text {th }}$ Int. Conf. Positron Annihilation (New York: North-Holland Publishing Co.).

[70] Consolati G. and Quasso F., 1990, "Anomalous magnetic quenching of quasi-positronium in solid octadecane”, J. Phys.: Condens. Matter, 2, 3941 - 3946.

[71] Conwell E.M., 1967, Solid State Physics - Supplement 9 (New York: Academic Press).

[72] Cook G.A. (ed.), 1961, Argon, Helium and the Rare Gases (New York: Interscience Publishers).

[73] Daniel T.B. and Stump R., 1959, "Positron annihilation in helium", Phys. Rev., 115(6), 1599 -1600 .

[74] Daniuk S., Kontrym-Sznajd G., Majsnerowski J., Šob M. and Stachowiak H., 1989, "Electronpositron interaction in metals: momentum dependence of HMC and ionic core enhancement factors", J. Phys.: Condens. Matter, 1, 6321 - 6326.

[75] Daniuk S., Šob M. and Rubaszek A., 199la, "Theoretical calculations of positron annihilation with rare-gas core electrons in simple and transition metals", Phys. Rev. B, 43(4), $2580-$ 2593.

[76] Daniuk S., Kontrym-Sznajd G., Šob M., Majsnerowski J. and Stachowiak H., 1992, "Positron annihilation with rare-gas core electrons: momentum dependence of enhancement factor" in [152], $627-630$.

[77] Darewych J.W., 1979, "Angular correlation of two-photon positron annihilation in hydrogen gas", Can. J. Phys., 57, $1027-1030$.

[78] De Graaf L.A. and Mozer B., 1971, "Structure study of liquid neon by neutron diffraction", J. Chem. Phys., 55(10), $4967-4973$.

[79] Deutsch M., 1951a, "Evidence for the formation of positronium in gases", Phys. Rev., 82, 455.

[80] Deutsch M., 1951b, "Three-quantum decay of positronium", Phys. Rev., 83, 866.

[81] Deutsch M. and Berko S., 1965, "Siegbahn K. (ed.)" in Alpha-, Beta- and Gamma-ray Spectroscopy, Amsterdam: North-Holland Publishing Co. (615),

[82] Dey T.H. and Lewis T.J., 1968, "Ion mobility and liquid motion in liquefied argon", J. Phys. $D, 1,1019-1029$.

[83] Dirac P.A.M., 1930, "On the annihilation of electrons and protons", Proc. Camb. Phil. Soc., 26, 361 .

[84] Dixon W.R. and Trainor E.H., 1955, "Nature of positron annihilation in liquids and solids", Phys. Rev., 97(3), 733 - 736.

[85] Dobbs E.R. and Jones G.O., 1957, "Theory and properties of solid argon", Rep. Prog. Phys., $20,51 \epsilon-564$. 
[86] Drachman R.J., 1968, "Variational bounds in positron-atom scattering", Phys. Rev., 173, 190 $-202$.

[87] Drachman R.J., 1969, "Addendum to 'Variational bounds in positron-atom scattering' ", Phys. Rev., 179(1), $190-202$.

[88] Dupasquier A., 1983, "Positroniumlike systems in solids" in [41], $510-564$.

[89] Dupasquier A., 1988, "Quasipositronium in liquids and solids" in [257], 485 - 506.

[90] Dupasquier A., De Natale P. and Rolando A., 1991, "Formal calculation of the pick-off annihilation rate for ortho- and parapositronium", Phys. Rev. B, 43(13), $10036-10041$.

[91] Edgell W.F., 1961, "Atomic structure and spectra" in [72], 97 - 155.

[92] Eldrup M., Vehanen A., Schultz P.J. and Lynn K.G., 1983, "Positronium formation and diffusion in a molecular solid studied with variable-energy positrons", Phys. Rev. Lett., 51(21), $2007-2010$.

[93] Eldrup M., Vehanen A., Schultz P.J. and Lynn K.G., 1985, "Positronium formation and diffusion in crystalline and amorphous ice using a variable-energy positron beam", Phys. Rev. B, 32(11), $7048-7064$.

[94] Emery L. and Song K.S., 1986, "Stability of electron bubbles in solid Ne, Ar and Kr", J. Phys. $C, 19,2469-2473$.

[95] Endt P.M., 1990, "Energy levels of $\mathbf{A}=21-44$ nuclei", Nucl. Phys., A521, $1-830$.

[96] Evans R.D., 1955, The Atomic Nucleus (Toronto: McGraw-Hill Book Co.).

[97] Feinberg G., Rich A. and Sucher J., 1990, "Quadratic Zeeman effect in positronium", Phys. Rev. A, 41(7), $3478-3480$.

[98] Ferrell R.A., 1956, "Theory of positron annihilation in solids", Rev. Mod. Phys., 28(3), 308 $-337$.

[99] Ferrell R.A., 1957, "Long lifetime of positronium in liquid helium", Phys. Rev., 108(2), 167 $-168$.

[100] Ferrell R.A., 1958, "Ortho-parapositronium quenching by paramagnetic molecules and ions", Phys. Rev., 110(6), $1355-1357$.

[101] Freeman G.R. (ed.), 1987, Kinetics of Nonhomogeneous Processes (Toronto: John Wiley \& Sons).

[102] Freeman G.R., 1987, "Ionization and charge separation in irradiated materials" in [101], 19 87.

[103] Frolov A.M. and Smith V.H. Jr., 1994, "One-photon annihilation in the $\mathrm{Ps}^{-}$ion and the angular $\left(\mathrm{e}^{-}, \mathrm{e}^{-}\right)$correlation in two-electron ions", Phys. Rev. A, 49(5), $3580-3585$.

[104] Fuochi P.G. and Freeman G.R., 1972, "Molecular structure effects on the free-ion yields and reaction kinetics in the radiolysis of the methyl-substituted propanes and liquid argon: electron and ion mobilities", J. Chem. Phys., 56(5), $2333-2341$.

[105] Gee N., Floriano M.A., Wada T., Huang S.S.-S. and Freeman G.R., 1985, "Ion and electron mobilities in cryogenic liquids: Argon, nitrogen, methane, and ethane", J. Appl. Phys., 57(4), $1097-1101$.

[106] Gidley D.W., Rich A., Sweetman E. and West D, 1982, "New precision measurements of the decay rates of singlet and triplet positronium", Phys. Rev. Lett., 49(8), $525-528$. 


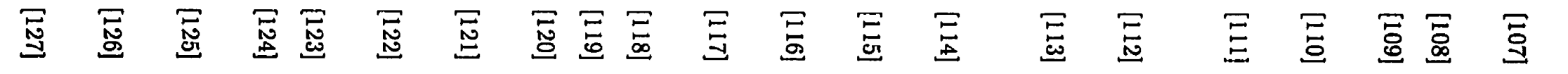

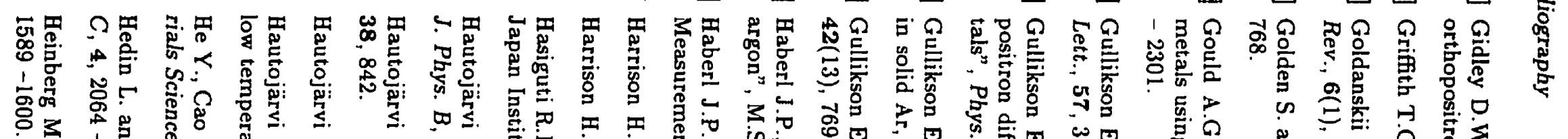

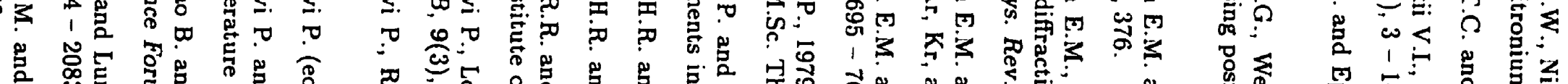

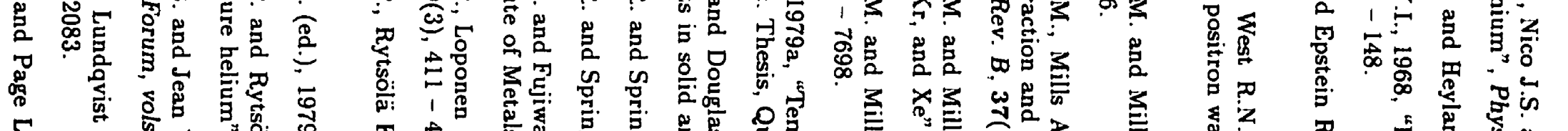

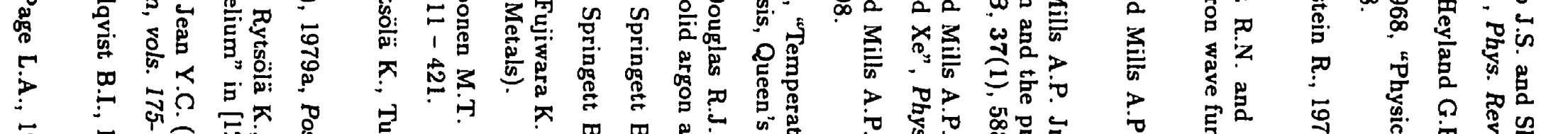

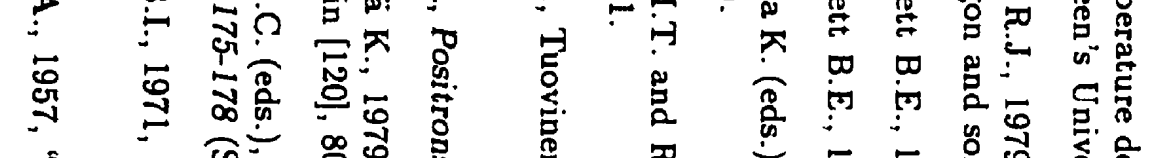

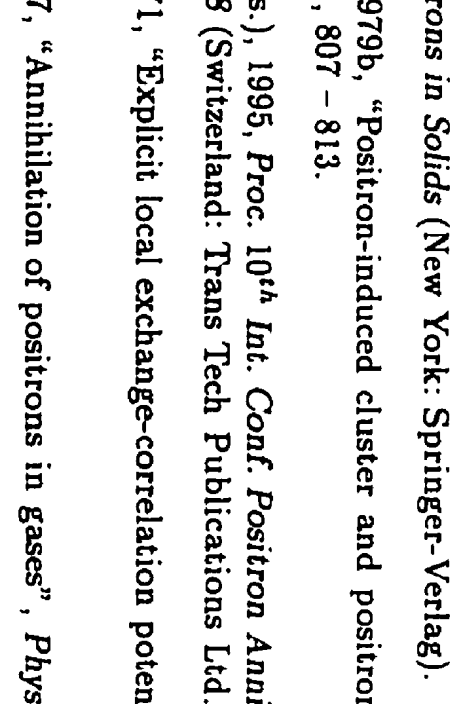

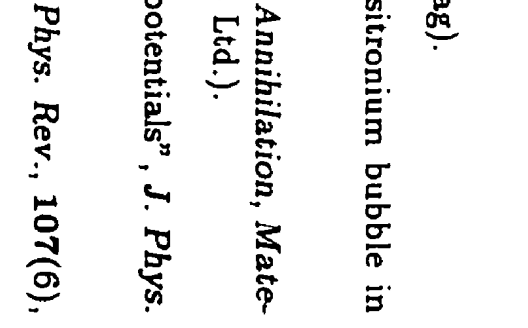

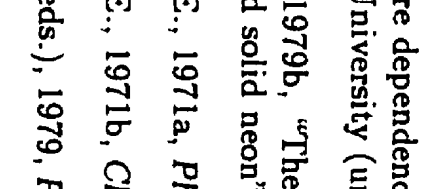

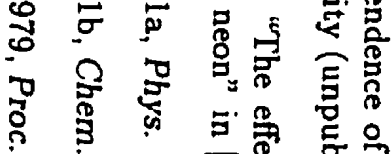

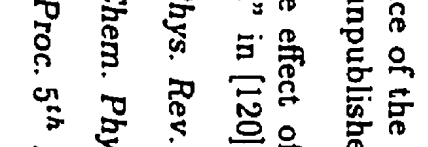

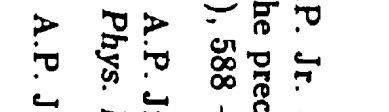

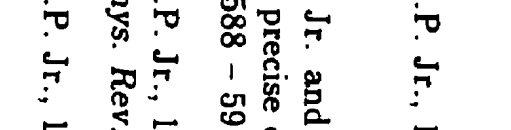

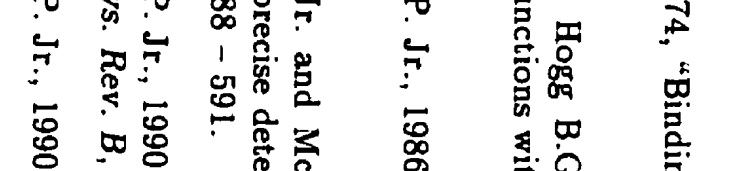

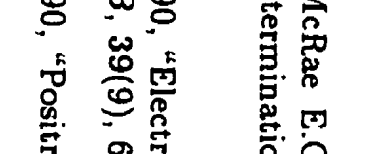

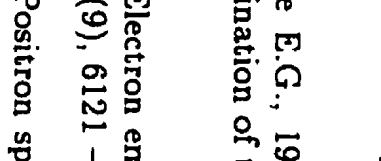

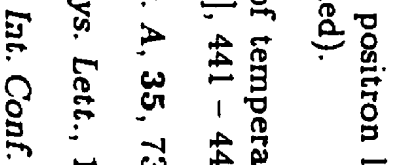

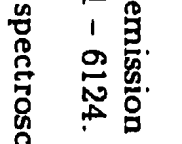

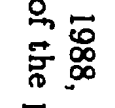
吕

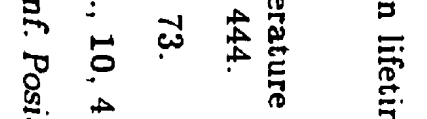
案 焉 䝛 . . :

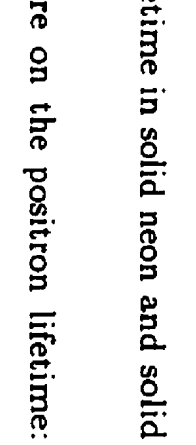

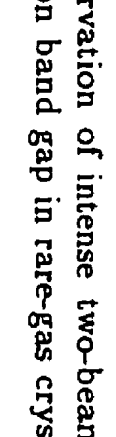

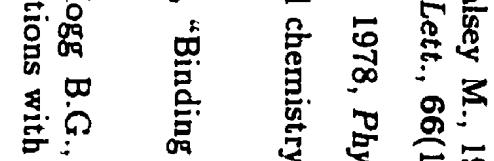

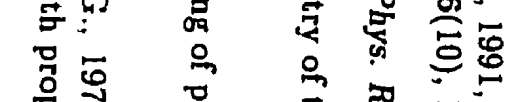

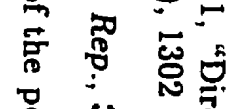

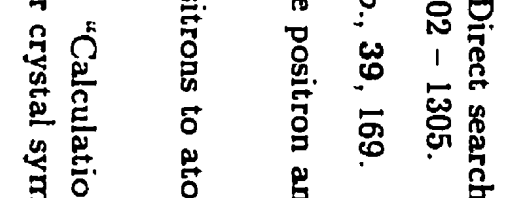

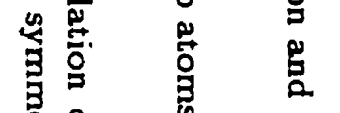
总음 品 穿

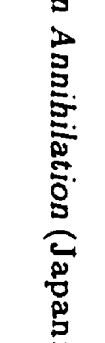

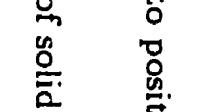
品 量

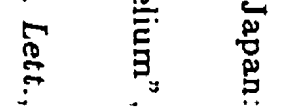

s. 
[128] Hegerberg R. and Crompton R.W., 1983, "Diffusion, attachment and attachment cooling of thermal electrons in oxygen and oxygen mixtures", Aust. J. Phys., 36, $831-844$.

[129] Herman F. and Skillman S., 1963, Atomic Structure Calculations (Toronto: Prentice-Hall of Canada Ltd.).

[130] Hernandez J.P. and Choi S.-I., 1969, "Positronium bubbles in liquid and solid helium-4", Phys. Rev., 188(1), $340-348$.

[131] Hernandez J.P., 1973, "Self-trapped states of an electron in a structurally disordered system", Phys. Rev. A, 7(5), $1755-1765$.

[132] Hernandez J.P., 1975, "Self-trapped states of electrons in dense fluids", Phys. Rev. B, 11(4), $1289-1296$.

[133] Hernandez J.P., 1976, "Self-trapping of ortho-positronium in dense fluid helium", Phys. Rev. $A, 14(4), 1755-1765$.

[134] Hernandez J.P., 1991, "Electron self-trapping in liquids and dense rases", Rev. Mod. Phys., $63(3), 675-697$.

[135] Hewson P.W., Jones G. and Vogt E.W., 1972, "On the interaction of positrons with argon", Can. J. Phys., 50, $821-825$.

[136] Heyland G.R, Charlton M., Griffith T.C. and Wright G.L., 1982, Can. J. Phys., 60, 503.

[137] Heyland G.R, Charlton M., Griffith T.C. and Clark G., 1985, "The temperature dependence of free positron lifetimes and positronium fractions in gaseous $\mathrm{CO}_{2}$ and $\mathrm{SF}_{6}{ }^{\prime}$, Chem. Phys., 95, $157-163$.

[138] Hodges C.H., McKee B.T.A., Triftshauser W. and Stewart A.T., 1972, "Umklapp annihilation of positronium in crystals", Can. J. Phys., 50, 103 - 109.

[139] Hogg B.G. and Stewart A.T. (eds.), 1971, Proc. $2^{\text {nd }}$ Int. Conf. Positron Annihilation (Kingston, Ontario, Canada).

[140] Humberston J.W., 1973, "The scattering of low energy positrons by helium", J. Phys. B, 6, L305 - L308.

[141] Humberston J.W., 1974, "Annihilation in positron-helium scattering", J. Phys. B, 7(9), L286 - L289.

[142] Humberston J.W. and McDowell M.R.C., 1984, Positron Scattering in Gases (New York: Plenum Press).

[143] Iakubov I.T. and Khrapak A.G., 1978, "On the annihilation rate of positronium in dense rare gases", Appl. Phys., 16, $179-183$.

[144] Iakubov I.T. and Khrapak A.G., 1982, "Self-trapped states of positrons and positronium in dense gases and liquids", Rep. Prog. Phys., 45, $697-751$.

[145] Ito $Y ., 1988$, "Radiation Chemistry: Intraspur effects and positronium formation mechanisms" in [248], $120-157$.

[146] Jackson J.D., 1975, Classical Electrodynamics (Toronto: John Wiley \& Sons, Inc.).

[147] Jain P.C., Singru R.M. and Gopinathan K.P. (eds.), 1985, Proc. $7^{\text {th }}$ Int. Conf. Positron Annihilation (Singapore: World Scientific Publ. Co.).

[148] Jarlborg T. and Singh A.K., 1987, "Local-density approach for calculation of electron-positron enhancement in transition metals", Phys. Rev. B, 369, 4660-4663. 
[149] Jauch J.M. and Rohrlich F., 1980, The Theory of Photon and Electrons (New York: SpringerVerlag).

[150] Jean Y.C., 1988, "The slow positron as a probe of surface chemistry" in [248], $240-253$.

[151] Kahana S., 1963, Phys. Rev., 129, 1622.

[152] Kajcsos Z. and Szeles C. (eds.), 1992, Proc. $9^{\text {th }}$ Int. Conf. Positron Annihilation, Materials Science Forum, vols. 105-110 (Switzerland: Trans Tech Publications Ltd.).

[153] Kakimoto M, Hyodo T., Chiba T., Akahane T. and Chang T.B., 1987, "Observation of tripletsinglet conversion of positronium via inelastic scattering by oxygen", J. Phys. B, 20, L107L113.

[154] Kakimoto M. and Hyodo T., 1988, "Evidence for normal formation of positronium in gaseous Xe", J. Phys. B, 21, $2977-2987$.

[155] Kakimoto M., Hyodo T. and Chang T.B., 1990, "Conversion of ortho-positronium in lowdensity oxygen gas", J. Phys. B, 23, $589-597$.

[156] Karl M.W., Nakanishi H. and Schrader D.M., 1984, "Chemical stability of positronic complexes with atoms and atomic ions", Phys. Rev. A, 30(4), $1624-1628$.

[157] Khrapak A.G., 1995, "Kinetics of positronium self-trapping in liquids" in [125], $699-702$.

[158] Kirkegaard P., Pedersen N.J. and Eldrup M., 1989, PATFIT-88: A Data-Processing System for Positron Annihilation Spectra on Mainframe and Personal Computers (Roskilde, Denmark: Risø National Laboratory report \#Risø-M-2740).

[159] Klein M.L. and Venables J.A. (eds.), 1976, Rare Gas Solids Volume I (New York: Academic Press).

[160] Klein M.L. and Venables J.A. (eds.), 1977, Rare Gas Solids Volume II (New York: Academic Press).

[161] Klobuchar R.L. and Karol P.J., 1980, "Positronium formation and quenching in argon-oxygen mixtures", J. Phys. C, 84, $483-488$.

[162] Kubica P. and Stott M.J., 1974, "Positron wavefunctions and energies in pure metals: A pseudopotential approach", J. Phys. F, 4, $1969-1981$.

[163] Kubica P., 1975a, "Positron wavefunctions and energies in pure metals, thermalization of positrons and positron effective mass", Ph.D. Thesis, Queen's University (unpublished).

[164] Kubica P., McKee B.T.A., Stewart A.T., and Stott M.J., 1975b, "Positron localization in Li-Mg alloys", Phys. Rev. B, 11(1), $11-22$.

[165] Kubica P. and Stewart A.T., 1975c, "Thermalization of positrons and positronium", Phys. Rev. Lett., 34(14), 852.

[166] Kuper C.G., 1961, "Theory of negative ions in liquid helium", Phys. Rev., 122(4), 1007 1011.

[167] LeComber P.G., Wilson J.B. and Loveland R.J., 1976, "Transport of excess charge carriers in liquid and solid hydrogen", Sol. St. Comm., 18, $377-380$.

[168] Lerner R.G. and Trigg G.L. (eds.), 1990, Encyclopedia of Physics (New York: VCH Publishers, Inc.).

[169] Lepage G.P., Mackenzie P.B., Streng K.H. and Zerwas P.M., 1983, "Multiphoton decays of positronium", Phys. Rev. A, 28(5), 3090-3091. 
[170] Leung C.H., McMullen T. and Stott M.J., 1976, "Can positrons self-trap in metals ?", J. Phys. F, 6(6), $1063-1071$.

[171] Levine J.L. and Sanders T.M. Jr., 1967, "Mobility of electrons in low-temperature helium gas", Phys. Rev., 154(1), $138-149$.

[172] Liberman D.A., Cromer D.T. and Waber J.T., 1971, Comput. Phys. Commun., 2, 107.

[173] Liu D.C. and Roberts W.K., 1962, "Positronium lifetime in condensed hydrogen", J. Phys. Chem. Solids, 23, 1337 - 1340.

[174] Liu D.C. and Roberts W.K., 1963a, "Positron annihilation in condensed gases", Phys. Rev., $130(6), 2322-2325$.

[175] Liu D.C. and Roberts W.K., 1963b, "Free-positron annihilation mean life in diatomic and rare gases in liquid and solid state", Phys. Rev., 132(4), $1633-1635$.

[176] Loucks T.L., 1967, Augmented Plane Wave Method (New York: W.A. Benjamin, Inc.).

[177] Loveland R.J., Le Comber P.G. and Spear W.E., 1972, "Experimental evidence for electronic bubble states in liquid Ne", Phys. Lett., 39A(3), $225-226$.

[178] Löwdin P.O., 1956, "Quantum theory of cohesive properties of solids", Adv. in Phys., 5(17), $1-172$.

[179] Manninen M. and Hautojärvi P., 1978, "Clustering of atoms around the positron and positive ions in gaseous $\mathrm{He}, \mathrm{Ne}$, and Ar", Phys. Rev. B, 17(5), $2129-2136$.

[180] Manuel A., 1983, "Positron annihilation using high-density multiwire chambers" in [41], 581 -596 .

[181] Manuzio G.E., 1966, "Mean life of positronium in liquid helium. Temperature and density effect", il Nuovo Cimento $B, 42(2), 1981$ - 1987.

[182] Mao A.C. and Paul D.A.L., 1975, "Positron scattering and annihilation in neon gas", Can. J. Phys., 53, 2406 - 2414.

[183] Marder S., Hughes V.W., Wu C.S. and Bennett W., 1956, "Effect of an electric field on positronium formation in gases: Experimental", Phys. Rev., 103(5), 1258 - 1265.

[184] Mattheiss L.F., 1964, "Energy bands for solid argon", Phys. Rev., 133(5A), 1399 - 1403.

[185] McEachran R.P., Morgan D.L., Ryman A.G. and Stauifer A.D., 1977, "Positron scattering from noble gases", J. Phys. B, 10(4), $663-677$.

[186] McEachran R.P., Ryman A.G. and Stauffer A.D., 1978a, "Positron scattering from neon", $J$. Phys. B, 11(3), $551-561$.

[187] McEachran R.P., Morgan D.L., Ryman A.G. and Stauffer A.D., 1978b, "Corrigendum and Addendum: Positron scattering from noble gases", J. Phys. B, 11(5), 951 - 953.

[188] McEachran R.P., Ryman A.G. and Campbell L.E.M., 1980, "Positron scattering from krypton and xenon", J. Phys. B, 13, $1281-1292$.

[189] McEachran R.P., Ryman A.G. and Stauffer A.D., 1979, "Positron scattering from argon", J. Phys. B, 12(6), $1031-1041$.

[190] McMullen T., 1985, "How positrons move: A review of the positron-phonon interaction and positron diffusion" in [147], $657-668$.

[191] Mijnarends P.E., 1969, "Finite slit-length correction in positron annihilation experiments", $J$. Appl. Phys., 40, 3027 - 3033. 
[192] Mills A.P. Jr., 1975, "Effects of collisions on the magnetic field quenching of positronium", J. Chem. Phys., 62(7), 2646 - 2659.

[193] Mills A.P. Jr., 1981, "Observation of positronium negative ion", Phys. Rev. Lett., 46(11), 717 -720 .

[194] Mills A.P. Jr., 1983a, "Measurement of the decay rate of the positronium negative ion", Phys. Rev. Lett., 50(9), 671 - 674.

[195] Mills A.P. Jr., 1983b, "Experimentation with low-energy positron beams" in [41], $432-509$.

[196] Mills A.P. Jr. and Gullikson E.M., 1986, "Solid neon moderator for producing slow positrons". Appl. Phys. Lett., 49(17), $1121-1123$.

[197] Mohorovičič S., 1934, Astron. Nacht., 253, 94.

[198] Mogensen O.E., 1970, "Discussion of the finite slit length error in positron annihilation experiments", Nucl. Instr. and Meth., 84, $293-296$.

[199] Mogensen O.E., 1974, "Spur reaction model of positronium formation", J. Chem. Phys., 60(3), $998-1004$.

[200] Mogensen O.E., Jacobsen F.M. and Eldrup M., 1982, "Comparison of ortho Ps and para Ps formation probabilities" in [69], $809-811$.

[201] Mogensen O.E., 1983, "On positronium yield enhancement in polyethylene in high electric fields", Phys. Lett., 96A(5), $250-254$.

[202] Mogensen O.E., 1986, "On positronium formation in crystalline and amorphous ice at low positron energy", Phys. Lett., 118A(7), $357-362$.

[203] Mokrushin A.D. and Goldanskii V.I., 1968, "Conversion of positronium atoms on $\mathrm{O}_{2}$ and NO molecules", Sov. Phys. JETP, 26(2), $314-317$.

[204] Nicolaides C.A. and Aspromallis G., 1991, "Binding of an electron by He and Xe", Phys. Rev. $A, 44(3), 2217-2219$.

[205] Nieminen R.M., Valimaa I., Manninen M. and Hautojärvi P., 1980, "Density-functional theory of positronium and electron bubbles in helium fluids", Phys. Rev. A, 21(5), $1677-1686$.

[206] Nieminen R.M., 1984, "Surface studies with slow positron beams" in [142], 139 - 154.

[207] Obenshain F.E. and Page L.A., 1962, "Strong electric field experiments on positronium formation in gases", Phys. Rev., 125(2), $573-581$.

[208] Oliva J., 1980, “New calculational scheme for particle range and an application to a positron entering a metal", Phys. Rev. B, 21, $4918-4934$.

[209] Onn D.G. and Silver M., 1971, "Injection and thermalization of hot electrons into solid, liquid, and gaseous helium at low temperatures", Phys. Rev. A, 3(5), $1773-1779$.

[210] Ore A. and Powell J.L., 1949, "Three-photon annihilation of an electron-positron pair", Phys. Rev., 75(11), 1696 - 1699.

[211] Orth P.H.R. and Jones G., 1969a, "Annihilation of positrons in argon I. Experimental", Phys. Rev., 183(1), $7-15$.

[212] Orth P.H.R. and Jones G., 1969a, "Annihilation of positrons in argon II. Theoretical", Phys. Rev., 183(1), $16-22$.

[213] Padmore T.C. and Cole M.W., 1974, "Free surface of He II and the electron bubble", Phys. Rev. $A, 9(2), 802-807$. 
[214] Page L.A. and Heinberg M., 1957, "Measurement of the longitudinal polarization of positrons emitted by sodium-22", Phys. Rev., 106(6), $1220-1224$.

[215] Paul D.A.L. and Graham R.L., 1957, "Annihilation of positrons in liquid helium", Phys. Rev., 106(1), $16-18$.

[216] Paul D.A.L., 1958, "Long $\tau_{2}$ lifetimes from positronium annihilations in liquid argon and liquid nitrogen", Can. J. Phys., 36(5), 640 - 642.

[217] Paul D.A.L., 1959, "Possible chemical reaction of orthopositronium with oxygen", Can. J. Phys., 37(9), $1059-1060$.

[218] Peker L.K., 1990, "Nuclear data sheets update for $A=58^{* ”}$, Nucl. Data Sheets, 61, 189 241.

[219] Pepe I., Paul D.A.L, Deutsch J. and Prieels R., 1993, "Positronium formation in liquid nitrogen as a function of electric field", J. Phys. B, 26, L373 - L378.

[220] Pepe I., Paul D.A.L, Steyaert J., Gimeno-Nogues F., Deutsch J. and Prieels R., 1995, "Positron annihialtion in liquid helium and liquid argon under electric field", J. Phys. B, 28, $3643-$ 3659 .

[221] Perkins A. and Carbotte J.P., 1970, "Effect of the positron-phonon interaction on positron motion", Phys. Rev. B, 1, 101.

[222] Pollack G.L., 1964, "The solid state of rare gases", Rev. Mod. Phys., 36, 748 - 791.

[223] Press W.H., Teukolsky S.A., Vetterling W.T. and Flannery B.P., 1992, Numerical Recipes in $C$ (New York: Cambridge University Press).

[224] Puska M.J., Mäkinen S., Manninen and Nieminen R.M., 1989, "Screening of positrons in semiconductors and insulators", Phys. Rev. B, 39(11), $7666-7679$.

[225] Puska M.J. and Nieminen R.M., 1992, "Positron and electron energy levels in rare-gas solids", Phys. Rev. B, 46(3), $1278-1283$.

[226] Ramanan G. and Freeman G.R., 1987, "Electron thermalization distances and free ion yields in liquid nitrogen from $77 \mathrm{~K}$ to near $T_{c}{ }^{n}, J$. Chem. Phys., 87(1), $319-324$.

[227] Ray A.K. and Trickey S.B., 1981, "Augmented-plane-wave to Gaussian-orbital conversion procedure: One-electron states and Compton profiles for fcc neon", Phys. Rev. B, 24(4), 1751 -1760 .

[228] Rich A., 1981, "Recent experimental advances in positronium research", Rev. Mod. Phys., 53(1), $127-165$.

[229] Ritter M.W., Egan P.O., Hughes V.W. and Woodle K.A., 1984, "Precision determination of the hyperfine-structure interval in the ground state of positronium. V", Phys. Rev. A, 30(3), $1331-1338$.

[230] Robkoff H.N. and Hallock R.B., 1982, "Structure-factor measurements in ${ }^{4} \mathrm{He}$ as a function of density", Phys. Rev. B, 25(3), $1572-1588$.

[231] Roellig L.O. and Kelly T.M., 1965, "Positron lifetimes in low-temperature helium gas", Phys. Rev. Lett., 15, 746 - 748.

[232] Roellig L.O. and Kelly T.M., 1967, "Cavity formation in helium", Phys. Rev. Lett., 18(11), $387-390$.

[233] Rohrlich F. and Carlson B.C., 1954, "Positron-electron differences in energy loss and multiple scattering", Phys. Rev., 93(1), 38- 44. 
[234] Rose K.L. and DeBenedetti S., 1965, "Positron annihilation in solid argon", Phys. Rev., 138(3A), $927-933$.

[235] Rotondi A., 1977, "The use of Fourier series in the deconvolution of experimental curves", Nucl. Instr. and Meth., 142, 499 - 506.

[236] Rubaszek A. and Stachowiak H., 1984a, "Application of quadratic response theory to the screening of a positron in an electron gas of metallic density", Phys. Status Solidi B, 124(159), $159-\mathrm{I} 66$.

[237] Rubaszek A., Stachowiak H., Boronski E. and Szotek Z., 1984b, “Electron-positron enhancement factors for an electron gas of high density within the Kahana formalism", Phys. Rev. B, 30(5), $2490-2497$.

[238] Rytsölä K., Rantapuska K. and Hautojärvi P., 1984a, "Positron annihilation and cluster formation in nitrogen fluid", $J$. Phys. $B, 17,299-317$.

[239] Rytsölä K., Vettenranta J. and Hautojärvi P., 1984b, "An experimental study of positronium bubbles in helium fluids", J. Phys. B, 17, $3359-3373$.

[240] Ruark A.E., 1945, "Positronium", Phys. Rev., 68, 278.

[241] Rubaszek A., Stachowiak H., Boronski E., and Szotek Z., 1984, "Electron-positron enhancement factors for an electron gas of high density within the Kahana formalism", Phys. Rev. B, $305,2490-2497$.

[242] Sakai Y., Bottcher H. and Schmidt W.F., 1982, "Excess electrons in liquid hydrogen, liquid neon, and liquid helium", J. of Electrostatics, 12, $89-96$.

[243] Salvadori A. and Carbotte J.P., 1969, "Theory of positron annihilation in inert-gas solids", Phys. Rev., 188(2), $550-556$.

[244] Schrader D.M. and Wang C.M., 1976, "Approximate molecular orbital theory for positrons and positronium atoms bound to molecules", J. Phys. C, 80(22), $2507-2518$.

[245] Schrader D.M., 1979, "Semiempirical polarization potential for low-energy positron-atom and positron-atomic-ion interactions. I. Theory: Hydrogen and the noble gases", Phys. Rev. A, 20(3), $918-932$.

[246] Schrader D.M. and Svetic R.E., 1982a, "The interaction of positrons with large atoms and molecules: Theory", Can. J. Phys., 60, $517-542$.

[247] Schrader D.M., Lowenschuss A., Jean J.C., Nakamoto K., and Pollard B.D., 1982b, "Positron Lifetime Spectra of Annealed Argon Matrices" in [69], 657 - 659.

[248] Schrader D.M. and Jean Y.C. (eds.), 1988, Positron and Positronium Chemistry (New York: Elsevier).

[249] Schwentner N., Koch E.-E. and Jortner J., 1985, Electronic Excitations in Condensed Rare Gases (New York: Springer-Verlag).

[250] Schultz P.J. and Lynn K.G., 1988, "Interaction of positron beams with surfaces, thin films, and interfaces", Rev. Mod. Phys., 60(3), $701-779$.

[251] Schwinber P.B., Van Dyck R.S. and Dehmelt H.G., 1981, "Trapping and thermalization of positrons for geonium spectroscopy", Phys. Lett., $81 \mathbf{A}(2,3), 119-120$.

[252] Seltzer S.M. and Berger M.J., 1984, "Improved procedure for calculating the collision stopping power of elements and compounds for electrons and positron", Int. J. Appl. Radiat. Isot., $35(7), 665-676$. 
[253] Sharma B.K., 1974, "Positron annihilation in solid krypton and xenon", Appl. Phys., 5, 265 $-267$.

[254] Sharma B.K. and Majumdar C.K., 1979, "Positron annihilation in inert-gas-solids" in [120], $8 ! 5-818$.

[255] Sharma B.K. and Singh H., 1982, "Enhancement effect on positron annihilation in solid argon and xenon" in [69], $645-647$.

[256] Sharma S.C., Eftekhari A. and MsNutt J.D., 1982, Phys. Rev. Lett., 48, 953.

[257] Sharma S.C. (ed.), 1988, Positron Annihilation Studies in Fluids (Singapore: World Scientific).

[258] Shearer J.W. and Deutsch M., 1949, Phys. Rev., 76, 462.

[259] Shimamori H. and Fessenden R.W., 1981, "Thermal electron attachment to oxygen and van der Waals molecules containing oxygen ${ }^{n}, J$. Chem. Phys., 74(1), $453-466$.

[260] Shimamori H. and Hotta $\mathrm{H} ., 1983$, "Mechanism of thermal attachment to $\mathrm{O}_{2}$ as studied by observing isotope effects of attachment rates for ${ }^{18} \mathrm{O}_{2}$ systems", J. Chem. Phys., 78(3), 1318 $-1324$.

[261] Shimamori $\mathrm{H}$. and Hotta $\mathrm{H}$., 1984, "Mechanism of thermal electron attachment to $\mathrm{O}_{2}$ : Isotope effect studies with ${ }^{18} \mathrm{O}_{2}$ in rare gases and some hydrocarbons", J. Chem. Phys., 81(3), 1271 $-1276$.

[262] Singh B., 1991, "Nuclear data sheets update for $A=64^{*}$, Nucl. Data Sheets, 62, $603-708$.

[263] Smejtek P., Silver M., Dy K.S. and Onn D.G., 1973, "Hot electron injection into dense argon, nitrogen and hydrogen", J. Chem. Phys., 59(3), 1374- 1384.

[264] Smith J.B. Jr., McGervey J.D. and Dahm A.J., 1977, "Orthopositronium decay in gaseous, liquid, and solid helium", Phys. Rev. B, 15(3), 1378 - 1387.

[265] Sob M., 1985a, "Electronic structure and positron annihilation in alkali metals: Isolation of ionic core contribution and valence high- momentum components", Sol. St. Comm., 53(3), $249-253$.

[266] Sob M., 1985b, "Systematics of the rare-gas core contributions to the positron annihilation spectra of some simple and transition metals", Sol. St. Comm., 53(3), $255-258$.

[267] Sowada U., Warman J.M. and de Haas M.P., 1982a, "Hot-electron thermalization in solid and liquid argon, krypton, and xenon", Phys. Rev. B, 25(5), $3434-3437$.

[268] Sowada U., Warman J.M. and de Haas M.P., 1982b, "The density dependence of hot-electron thermalization in liquid argon, krypton, and xenon", Chem. Phys. Lett., 90(3), $239-241$.

[269] Spektor D.M. and Paul D.A.L., 1971a, "Positronium formation in liquid argon and in liquid nitrogen, and the effect of adding traces of oxygen" in [139], 5.71-5.74.

[270] Spektor D.M., Paul D.A.L. and Stevens J.R., 1971b, "Positron and positronium lifetimes in liquid argon", Can. J. Phys., 49, $939-941$.

[271] Stampfii P. and Bennemann K.H., 1991, "Theoretical results for the density dependence of the electron affinity of nonpolar liquids $\mathrm{Ar}, \mathrm{Kr}$, and Xe", Phys. Rev. A, 44(12), $8210-8214$.

[272] Steinbacher J.J. and Stewart A.T., 1992a, "Magnetic quenching experiments in liquid rare gases" in [152], $1711-1714$.

[273] Steinbacher J.J. and Stewart A.T., 1992b, "On the broad component in condensed rare gases" in [152], $1715-1720$.. 
[274] Steinbacher J.J. and Stewart A.T., 1995, "Positronium yields in liquid argon and nitrogen" in [125], $777-780$..

[275] Stewart A.T. and Roellig L.O., 1967a, Positron Annihilation (New York: Academic Press).

[276] Stewart A.T. and Briscoe C.V., 1967b, "Positron annihilation in liquid helium" in [275], 383 -386 .

[277] Stewart A.T., Briscoe C.V. and Steinbacher J.J., 1990, "Positron annihilation in simple condensed gases", Can. J. Phys., 68, 1362 - 1376.

[278] Stott M.J. and Kubica P., 1975, "New approach to the positron distribution in metals and alloys", Phys. Rev. B, 11(1), 1 - 10.

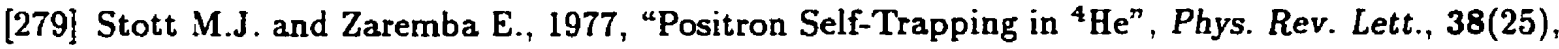
$1493-1496$.

[280] Stott M.J. and West R.N., 1978, "The positron density distribution in metals: Temperature effects", J. Phys. F, 8(4), $635-650$.

[281] Stroscio M.A., 1975, "Positronium: A review of the theory", Phys. Rep., 22C, $216-277$.

[282] Stroud D. and Ehrenreich H., 1968, "Assumption-independent single-particle wave functions for positrons in solids: Applications to angular distributions in $\mathrm{Al}$ and $\mathrm{Si}^{\prime \prime}$, Phys. Rev., 171(2), $399-407$.

[283] Swan D.W., 1963, "Electron attachment processes in liquid argon containing oxygen or nitrogen impurity", Proc. Phys. Soc., 82, $74-84$.

[284] Swan D.W., 1964, "Drift velocity of electrons in liquid argon, and the influence of molecular impurities", Proc. Phys. Soc., 83, $659-666$.

[285] Tachiya M. and Schmidt W.F., 1989, "Escape probability of germinate electron-ion recombination in the limit of large electron mean free path", J. Chem. Phys., 90(4), $2471-2475$.

[286] Tao S.J., Green J.H. and Celitans G.J., 1963, "Thermalization of positrons in argon", Proc. Phys. Soc., 81, $1091-1100$.

[287] Tao S.J., 1972, "Positronium annihilation in molecular substances", J. Chem. Phys., 56(11), $5499-5510$.

[288] Teutsch W.B. and Hughes V.W., 1956, "Effect of an electric field on positronium formation in gases: Theoretical", Phys. Rev., 103(5), 1266 - 1281.

[289] Theriot E.D. Jr., Beers R.H. and Hughes V.W., 1967, "Precision redetermination of the hyperfine structure interval of positronium", Phys. Rev. Lett., 18(19), $767-769$.

[290] Thorlacius A.E. and Cooper E.D., 1987, "An algorithm for integrating the Schrödinger equation", J. Comp. Phys., 72, $70-77$.

[291] Triftshäuser W., Legg J., Stewart A.T. and Briscoe C.V., 1969, "Zero-point bubbles in liquid and solid helium" in Allen J.J., Finlayson D.M. and McCall D.M. (eds.), Proc. $11^{\text {th }}$ Int. Conf. on Low Temperature Physics (St. Andrews, Scotland: University of St. Andrews), $304-307$.

[292] Tuomisaari M., Rytsölä K. and Hautojärvi P., 1985, "Localized state of positron in argon", Phys. Lett., 112A, $279-282$.

[293] Tuomisaari M., Rytsölä K., Nieminen R.M. and Hautojärvi P., 1986, "Localisation of orthopositronium in argon", J. Phys. B, 19, $2667-2681$.

[294] Tuomisaari M., Rytsölä K., Nieminen R.M. and Hautojärvi P., 1988, "Positron annihilation in xenon", J. Phys. B, 21, $3917-3928$. 
[295] Tuomisaari M., Howell R.H. and McMullen T., 1989, "Spectroscopy of positronium emission from alkali halides", Phys. Rev. B, 40(4), $2060-2069$.

[296] Tuomisaari M., Howell R.H. and McMullen T., 1991, "Positronium formation and diffusion in the rare-gas solids", J. Phys. B, 24, $1455-1466$.

[297] Van Dyck R.S., Schwinberg P.B. and Dehmelt H.G., 1987, "New high-precision comparison of electron and positron $g$ factors", Phys. Rev. Lett., 59(1), $26-29$.

[298] Varlashkin P.G., 1971, "Positron annihilation and positronium bubbles in liquid and solid argon, krypton, and xenon", Phys. Rev. A, 3(4), 1230 - 1232.

[299] Venables J.A. and Smith B.L., 1977, "Crystal growth and crystal defects" in [160], $609-661$.

[300] Wackerle J. and Stump R., 1957, "Annihilation of positrons in liquid helium", Phys. Rev., 106(1), $18-20$.

[301] Wada T. and Freeman G.R., 1980, "Electron localization in dense nitrogen vapor", J. Chem. Phys., 72(12), $6726-6730$.

[302] Wallace P.R., 1960, "Positron annihilation in solids and liquids" in , Solid State Physics, 10. (New York: Academic Press), 1 - 69.

[303] Ward N.J. and Tuli J.K., 1986, "Nuclear data sheets for $A=65^{* \prime}$, Nucl. Data Sheets, 47 , $135-260$.

[304] West R.N., 1973, "Positron studies of condensed matter", Adv. in Phys., 22, $263-383$.

[305] Westbrook C.I., Gidley D.W., Conti R.S. and Rich A., 1989, "Precision measurement of the orthopositronium vacuum decay rate using the gas technique", Phys. Rev. A, 40(10), $5489-$ 5499 .

[306] Wheeler J.A., 1946, Ann. Phys. (N.Y.), 48, 219.

[307] Woll E.J. Jr. and Rose K.L., 1966, "Theory of positron lifetime in solid argon: The effect of correlation", Phys. Rev., 145(1), 268 - 273.

[308] Wright G.L., Charlton M., Griffith T.C. and Heyland G.R., 1985, "The annihilation of positrons and positronium formation in gaseous $\mathrm{Kr}$ and Xe", J. Phys. B, 18, $4327-4347$.

[309] Wu S.-T. and Yan G.-S., 1982, "Surface tension of simple liquids", J. Chem. Phys., 77(11), $5799-5801$.

[310] Yarnell J.L., Katz M.J., Wenzel R.G., and Koenig S.H., 1973, "Structure factor and radial distribution function for liquid argon at $85^{\circ} \mathrm{K}^{\prime \prime}$, Phys. Rev. $A, 7(6), 2130-2144$. 


\section{Appendix A \\ Properties of the Condensed Gases}

This appendix gives a brief summary of the useful physical properties of the condensed rare gases and the diatomic $\mathrm{H}_{2}, \mathrm{~N}_{2}$ and $\mathrm{O}_{2}$. More thorough discussions on the rare gases can be found in several books (Cook 1961; Klein and Venables 1976, 1977; Schwenter et al. 1985) and well known reviews (Dobbs and Jones 1957; Pollack 1964).

In their atomic state the rare gases are characterized by their completely filled electronic shells. This highly stable configuration is the primary reason for their inert properties. It also accounts for their very high first ionization energies, $E_{\text {ion }}$. Since rare gas atoms possess no permanent dipole moment the average charge distribution is spherically symmetric, in the absence of an electric field. The response of an atom to an electric field is described by the atomic polarizability, $\alpha_{\mathrm{at}}$. Table A.1 lists some of the atomic properties of the rare gases. Table A.2 lists similar properties for the diatomic gases.

The rare gases form relatively simple liquids and solids because the principle binding force between atoms is the weak van der Waals force. The liquid properties are summarized in Tables A.3 and A.4. The heavier rare-gas solids all form face-centred cubic lattices (solid helium is hexagonal-close packed but only forms at pressures greater than $25 \mathrm{~atm}$.). They are excellent examples of tight binding solids with charge distributions that are only weakly perturbed from the atomic state. Table A.5 summarizes some of the solid-state properties of the heavier rare gases. 
Table A.1: A summary of the useful atomic rare-gas properties. $E_{\mathrm{x}}$ is the energy of the lowest excited state accessible from the ground state via a electric dipole transition. The lower Ore gap estimate is obtained by assuming that Ps formation does not compete effectively with inelastic excitations. The upper Ore gap estimate is obtained by assuming that the excitation cross-section is negligible compared to that for Ps formation above the excitation threshold. The threshold energies and theoretical estimate of the Ore gap Ps yield is from Schrader and Svetic (1982a). The rest of the Ps yield information is from Charlton et al. (1992).

\begin{tabular}{lccccc}
\hline \hline & $\mathrm{He}^{4}$ & $\mathrm{Ne}$ & $\mathrm{Ar}$ & $\mathrm{Kr}$ & $\mathrm{Xe}$ \\
\hline Atomic number, Z & 2 & 10 & 18 & 36 & 54 \\
Mass (amu) & 4.0026 & 20.183 & 39.948 & 83.80 & 131.20 \\
Atomic polarizability, $\alpha\left(\AA^{3}\right)$ & 0.2049 & 0.3946 & 1.639 & 2.481 & 4.044 \\
& & & & & \\
Threshold energies (eV ) & & & & & \\
First ionization energy, $E_{\text {ion }}$ & 24.59 & 21.56 & 15.76 & 14.00 & 12.13 \\
Excitation energy, $E_{\mathrm{x}}$ & 21.22 & 16.67 & 11.62 & 10.03 & 8.44 \\
Lowest excition energy & 19.82 & 16.62 & 11.55 & 9.92 & 6.77 \\
Ps threshold, $E_{\text {ion }}-6.8 \mathrm{eV}$ & 17.79 & 14.76 & 8.96 & 7.20 & 5.33 \\
& & & & & \\
Ps fraction & & & & & \\
Lower Ore estimate & 0.14 & 0.09 & 0.17 & 0.20 & 0.26 \\
Upper Ore estimate & 0.28 & 0.32 & 0.43 & 0.49 & 0.56 \\
Theoretical estimate & 0.23 & 0.25 & 0.33 & 0.38 & 0.44 \\
Observation & 0.23 & 0.26 & 0.33 & 0.11 & 0.03 \\
& & & & & \\
Positron scattering length $(\AA)$ & -0.205 & -0.289 & -2.175 & & \\
\hline \hline
\end{tabular}


Table A.2: A summary of the useful diatomic-gas properties. The lowest excited state that the positron can generate must have the same spin multiplicity as the ground state and requires an energy $E_{\mathrm{x}}$. The threshold energies and Ps fraction information is taken from Schrader and Svetic (1982a).

\begin{tabular}{|c|c|c|c|}
\hline & $\mathrm{H}_{2}$ & $\mathrm{~N}_{2}$ & $\mathrm{O}_{2}$ \\
\hline Mass (amu) & 4.0026 & 20.183 & 39.948 \\
\hline \multicolumn{4}{|l|}{ Threshold energies (eV) } \\
\hline First ionization energy, $E_{\text {ion }}$ & 15.45 & 15.57 & 12.07 \\
\hline Excitation energy, $E_{\mathrm{x}}$ & $11.37\left(B^{1} \Sigma_{u}^{+}\right)$ & $8.59\left(a^{1} \Pi_{g}\right)$ & $4.48\left(A^{3} \Sigma_{u}^{+}\right)$ \\
\hline Lowest excitation energy & $11.37\left(B^{1} \Sigma_{u}^{+}\right)$ & $8.59\left(a^{1} \Pi_{g}\right)$ & $0.98\left(a^{1} \Delta_{g}\right)$ \\
\hline Ps threshold, $E_{\text {ion }}-6.8$ & 8.65 & 8.77 & 5.27 \\
\hline Dissociation energy $(\mathrm{eV})$ & 4.48 & 9.76 & 5.12 \\
\hline \multicolumn{4}{|l|}{ Ps fraction } \\
\hline Lower Ore estimate & 0.18 & 0 & 0 \\
\hline Upper Ore estimate & 0.44 & 0.44 & 0.56 \\
\hline Observation & 0.45 & 0.19 & 0.32 \\
\hline \multicolumn{4}{|c|}{ Energy loss per collision ( $\mathrm{meV}$ ) } \\
\hline Elastic & 5 & 0.4 & 0.3 \\
\hline Rotational excitation & 43 & 1.5 & 1.1 \\
\hline Vibrational excitation & 545 & 293 & 196 \\
\hline Electron affinity (eV) & & & $0.440 \pm 0.008$ \\
\hline
\end{tabular}

Table A.3: A summary of the useful rare-gas liquid properties.

\begin{tabular}{lccccc}
\hline \hline & $\mathrm{He}^{4}$ & $\mathrm{Ne}$ & $\mathrm{Ar}$ & $\mathrm{Kr}$ & $\mathrm{Xe}$ \\
\hline Normal boiling point $(\mathrm{K})$ & 4.215 & 27.07 & 87.27 & 119.81 & 165.06 \\
Density at n.b.p. $\left(\mathrm{g} / \mathrm{cm}^{3}\right)$ & 0.1249 & 1.207 & 1.3998 & 2.413 & 3.057 \\
& & & & & \\
Surface tension $\left(\times 10^{-3} \mathrm{~N} / \mathrm{m}\right)[309]$ & 0.096 & 4.47 & 12.42 & 15.49 & 17.46 \\
& & & & & \\
Spur electron properties & & & & & \\
Affinity at n.b.p., $E_{A e}(\mathrm{eV})[271]$ & -1.05 & -0.67 & 0.20 & 0.45 & 0.61 \\
Mobility, $\mu_{e}\left(\mathrm{~cm}^{2} / \mathrm{V} \cdot \mathrm{s}\right)[101]$ & 0.020 & 0.0016 & 475 & 1900 & 2200 \\
Total ion yield, $\mathrm{G}_{\mathrm{tot}}^{\circ}[101]$ & & & 4.5 & 6.0 & 6.6 \\
Free ion yield, $\mathrm{G}_{\mathrm{f}}^{\circ}[101]$ & & & 2.7 & 4.0 & 4.4 \\
Thermalization time, $t_{t h}(\mathrm{ps})$ & $\sim 1[209]$ & & $900[267]$ & $4400[267]$ & $6500[267]$ \\
Thermalization distance, $b(\AA)[101]$ & & & $>2470$ & $>2030$ & $>1340$ \\
\hline \hline
\end{tabular}


Table A.4: A summary of the useful diatomic liquid properties.

\begin{tabular}{lccc}
\hline \hline & $\mathrm{H}_{2}$ & $\mathrm{~N}_{2}$ & $\mathrm{O}_{2}$ \\
\hline Surface tension at n.b.p. $\left(\times 10^{-3} \mathrm{~N} / \mathrm{m}\right)$ & 1.91 & 8.8 & \\
& & & \\
Spur electron properties & & & \\
Mobility, $\mu_{e}\left(\mathrm{~cm}^{2} / \mathrm{V} \cdot \mathrm{s}\right)$ & $0.017[101]$ & $0.0027[226]$ & \\
Total ion yield, $\mathrm{G}_{\mathrm{tot}}^{\circ}$ & & $2.9[226]$ & $\sim 4[104]$ \\
Free ion yield, $\mathrm{G}_{\mathrm{f}}^{\circ}$ & & $0.0034[226]$ & $0.013[104]$ \\
Thermalization time, $t_{\mathrm{th}}(\mathrm{ps})[263]$ & $\sim 0.2$ & $\sim 2$ & \\
Thermalization distance, $b(\AA)$ & & $55[226]$ & $110[104,101]$ \\
\hline
\end{tabular}


Table A.5: A summary of the useful rare-gas solid and solid $\mathrm{N}_{2}$ properties. The electron properties were all taken from Schwentner et al. (1985). $E_{z}$ is the exciton formation threshold. $E_{a}$ is the electron affinity. $E_{\mathrm{ph} \text { max }}$ are the maximum phonon energies. The positron properties were taken from the slow positron beam experiments of Gullikson and Mills $(1986,1989,1990)$. $E_{\text {Bragg }}$ defines the centre of the lowest positron band gap along the (111) direction. $E_{\text {gap }}$ is the with of that band gap. $\phi_{+}$is the positron work function or affinity. $\lambda_{+}$is an estimate of the positron mean free path. $E_{\mathrm{Ps}_{\mathrm{b}}}$ is the Ps binding energy while $\phi_{\mathrm{Ps}}$ is its work function. $\delta E$ is the measured average positron energy loss per collision below the elastic scattering threshold energy $E_{\text {el,th }}$ and the Ps formation threshold $E_{\mathrm{Ps}, \mathrm{b}}$.

\begin{tabular}{|c|c|c|c|c|c|}
\hline & $\mathrm{Ne}$ & $\mathrm{Ar}$ & $\mathrm{Kr}$ & $\mathrm{Xe}$ & $\mathrm{N}_{2}$ \\
\hline Triple point (K) & 24.55 & 83.78 & 115.95 & 161.3 & \\
\hline Density at t.p. $\left(\mathrm{g} / \mathrm{cm}^{3}\right)$ & 1.444 & 1.623 & 2.826 & 3.399 & \\
\hline Bulk modulus $\left(10^{9} \mathrm{~N} / \mathrm{m}^{2}\right)$ & 1.1 & 2.7 & 3.5 & 3.6 & \\
\hline Lattice constant $(\AA)$ & 4.536 & 5.470 & 5.820 & 6.353 & \\
\hline Nearest neighbour distance $(\AA)$ & 3.207 & 3.868 & 4.115 & 4.492 & \\
\hline \multicolumn{6}{|l|}{ Electronic properties } \\
\hline$E_{\text {gap }}^{\mathbf{a}}$ & 21.58 & 14.16 & 11.61 & 9.33 & 15.1 \\
\hline$E_{\mathrm{x}}(\mathrm{eV})$ & 16.5 & 12.1 & 10.25 & 8.36 & \\
\hline$E_{\mathrm{a}}(\mathrm{eV})$ & $-1.3(4 K)$ & $-0.4(6 \mathrm{~K})$ & 0.3 & 0.4 & \\
\hline$E_{\mathrm{ph}, \max }(\mathrm{meV})$ & & 8.3 & 6.2 & 5.4 & \\
\hline \multicolumn{6}{|l|}{ Spur electron properties } \\
\hline Mobility, $\mu_{e}\left(\mathrm{~cm}^{2} / V \cdot s\right)^{b}$ & 600 & 1000 & 3700 & 4500 & \\
\hline \multicolumn{6}{|l|}{ Positronic properties } \\
\hline$E_{\text {Bragg }}(\mathrm{eV})$ & $5.0(1)$ & $2.45(5)$ & $1.60(5)$ & $0.75(5)$ & \\
\hline$E_{\text {gap }}(\mathrm{eV})$ & $1.60(10)$ & $1.50(5)$ & $1.30(5)$ & $1.05(5)$ & \\
\hline$\phi_{+}=-V_{0}(\mathrm{eV})$ & $0.6(1)$ & $1.55(5)$ & $2.00(5)$ & $2.30(5)$ & $1.31(10)$ \\
\hline$\lambda_{+}(\AA)$ & $340(140)$ & $230(90)$ & $200(80)$ & $80(30)$ & $200(40)$ \\
\hline $\begin{array}{l}E_{\mathrm{Ps}, \mathrm{th}}(\mathrm{eV}) \\
E_{\mathrm{el} \text { th }}(\mathrm{eV})\end{array}$ & & $9.95(5)$ & $7.9(1)$ & $5.67(5)$ & $\begin{array}{c}10.0(5) \\
8.385(10)\end{array}$ \\
\hline$E_{\mathrm{Ps}, \mathrm{b}}(\mathrm{eV})$ & 4.5 & $2.8(1)$ & $1.7(1)$ & $1.45(10)$ & $3.8(5)$ \\
\hline$\phi_{P_{s}}(e V)$ & & $-2.8(1)$ & $-2.8(1)$ & $-2.8(1)$ & \\
\hline$\delta E(\mathrm{meV})$ & & $6(2)$ & $3(1)$ & $3(1)$ & $30(5)$ \\
\hline
\end{tabular}




\section{Appendix B}

\section{Positron Properties}

Table B.1 lists some of the physical properties of the positron and compares them to those of the electron. The $g$-factor anomaly $a$ is given by $g=2(1+a)=\frac{2 \mu}{\mu_{\theta}}$ where $g$ and $\mu$ are the $g$-factor and magnetic moment, respectively, and $\mu_{B}=\frac{e \hbar}{2 m_{0} c}$ is the Bohr magneton. The results are identical to within the accuracies achieved so far, as expected from $C P T$ invariance.

The most common way of injecting positrons into a sample is to use a radioactive source. The energy spectrum of the emitted positrons is continuous and typical for that of $\beta$-decay, with a kinetic energy end point $E_{\max }$ of about half to one $\mathrm{MeV}$. The average initial positron energy $E_{\text {ave }}$ is typically about $0.4 E_{\max }$. Most radioisotopes are produced by bombarding stable nuclei with protons or deuterium. ${ }^{64} \mathrm{Cu}$ and ${ }^{65} \mathrm{Zn}$ can also be produced quite effectively in a nuclear reactor due to the high thermal neutron capture cross-sections of stable ${ }^{63} \mathrm{Cu}$ and ${ }^{64} \mathrm{Zn}$. For positron lifetime experiments ${ }^{22} \mathrm{Na}$ is a common choice for a number of reasons: the prompt $\gamma$-ray (emitted by the nucleus $\sim 5$ ps after the positron) is required as a 'start' signal for timing purposes; the high fraction of positron decays reduces the background counts; and the long half-life is convenient. For angular correlation and doppler-broadening experiments the prompt $\gamma$-ray is not required and so other sources are also commonly used. Figure B.I shows the decay modes for ${ }^{22} \mathrm{Na}$, and for ${ }^{64} \mathrm{Cu}$, which was used in the angular-correlation experiments in this work.

In $§ 2.5 .1$ the annihilation of a slow, free electron-positron pair was discussed. It was mentioned that the pair typically annihilates from either a spin-singlet state $\left({ }^{1} S_{0}\right)$ with an even number of photons or from a spin-triplet state $\left({ }^{3} S_{1}\right)$ with an odd number of photons. This is a result of the $C P$ invariance of electromagnetic interactions. The charge-conjugation operator $\dot{\mathbf{C}}$ interchanges the particles and antiparticles when applied to a system and has eigenvalues $C$ called the charge parity. The spatial-inversion operator $\dot{\mathbf{P}}$ has eigenvalues $P=(-1)^{L} P_{i}$ called the spatial parity, where $L$ is the total orbital momentum and $P_{i}$ is the internal parity. For a particle-antiparticle pair $P_{i}=-1$. Since the system consists of an even number of spin $\frac{1}{2}$ particles $\hat{\mathbf{C}}$ and $\hat{\mathbf{P}}$ commute: $\hat{\boldsymbol{\Sigma}}=\hat{\mathbf{C}} \dot{\mathbf{P}}=\hat{\mathbf{P}} \dot{\mathbf{C}} . \hat{\boldsymbol{\Sigma}}$ has the effect of interchanging the electron and positron spins and has eigenvalues $\Sigma=C P=(-1)^{S+1}$ 
Table B.1: The physical properties of a positron and a comparison to those of an electron. The values in column 2 are the 'best' experimentally measured results and, as shown in column 3, are the same as the electron's to within the accuracies achieved so far. Experimental uncertainties are given in brackets.

\begin{tabular}{|c|c|c|c|}
\hline Property & & & Ref. \\
\hline Mass & $\begin{array}{l}9.1093897(54) \times 10^{-31} \mathrm{~kg} \\
\quad=511.0034(14) \frac{\mathrm{keV}}{e^{2}}\end{array}$ & $\frac{m^{-}}{m^{+}}-1< \pm 1.3 \times 10^{-7}$ & $\begin{array}{c}{[168,251]} \\
{[3]}\end{array}$ \\
\hline Charge & $1.60217733(49) \times 10^{-19} \mathrm{C}$ & & [168] \\
\hline Charge-to-mass ratio & $1.7588047(49) \times 10^{11} \frac{\mathrm{C}}{\mathrm{kg}}$ & $\frac{\mathrm{e}^{-} \mid / \mathrm{m}^{-}}{\mathrm{e}+\mid / \mathrm{m}^{-}}-1< \pm 1.3 \times 10^{-7}$ & {$[168]$} \\
\hline$g$-factor anomaly & $0.0011596521879(43)$ & $\frac{a^{-}}{a^{+}}-1=0.4(7.5) \times 10^{-9}$ & [297] \\
\hline$g$-factor & $2.0023193043758(86)$ & $\frac{2^{-}}{g^{+}}-1=0.5(2.1) \times 10^{-12}$ & {$[297]$} \\
\hline$\mu^{+} / \mu_{B}$ & $1.0011596521879(43)$ & $\frac{\mu^{-}}{\mu^{+}}-1=0.5(2.1) \times 10^{-12}$ & {$[297]$} \\
\hline
\end{tabular}

Table B.2: Annihilation properties of some common positron sources.

\begin{tabular}{lcccccc}
\hline \hline Isotope & Half-life & $\begin{array}{c}\text { Positrons per } \\
\text { decay }(\%)\end{array}$ & $\begin{array}{c}E_{\max } \\
(\mathrm{MeV})\end{array}$ & $\begin{array}{c}E_{\text {ave }} \\
(\mathrm{MeV})\end{array}$ & $\begin{array}{c}\text { Prompt } \gamma \\
\text { energy }(\mathrm{MeV})\end{array}$ & Ref. \\
\hline${ }^{11} \mathrm{C}$ & $20.39 \mathrm{~m}$ & 99 & 0.960 & & - & {$[4]$} \\
${ }^{22} \mathrm{Na}$ & $2.609 \mathrm{y}$ & 90.205 & 0.544 & 0.26 & 1.2745 & {$[95]$} \\
${ }^{44} \mathrm{Ti}\left({ }^{44} \mathrm{Sc}\right)$ & $49 \mathrm{y}$ & 88 & 1.475 & & 1.1570 & {$[95]$} \\
${ }^{57} \mathrm{Ni}$ & $35.65 \mathrm{~h}$ & 42.9 & 0.866 & & 1.3776 & {$[49]$} \\
${ }^{58} \mathrm{Co}$ & $70.83 \mathrm{~d}$ & 14.96 & 0.474 & 0.24 & 0.8108 & {$[218]$} \\
${ }^{64} \mathrm{Cu}$ & $12.700 \mathrm{~h}$ & 17.86 & 0.653 & 0.28 & - & {$[262]$} \\
${ }^{65} \mathrm{Zn}$ & $243.9 \mathrm{~d}$ & 1.415 & 0.329 & & - & {$[303]$} \\
${ }^{68} \mathrm{Ge}\left({ }^{68} \mathrm{Ga}\right)$ & $270.84 \mathrm{~d}$ & 89.12 & 1.899 & 0.81 & - & {$[25]$} \\
\hline \hline
\end{tabular}


called the spin parity, where $S$ is the total spin angular momentum. The charge parity for an electron-positron pair becomes

$$
C=(-1)^{L+S} .
$$

From the uncertainty principle one can deduce that for a $2 \gamma$ annihilation the electronpositron pair must be within $\sim 10^{-13} \mathrm{~m}$ of each other which amounts to contact on the atomic scale. The short range of the annihilation interaction means that slow electronpositron pairs can annihilate at a reasonable rate only if they are in a relative $s$-state $(L=0)$. For higher orbitals the electron and positron wave function overlap is negligible. The pair therefore annihilates from either a ${ }^{1} S_{0}(S=0)$ or a ${ }^{3} S_{1}(S=1)$ state. A photon has a negative charge parity so for a system of $n$ photons $C=(-1)^{n}$. The conservation of charge parity, which follows from $C P$ invariance, forces the singlet state to annihilate into an even number of photons and the triplet state into an odd number of photons. The total spin-averaged electron-positron pair annihilation rate is

$$
\lambda=\lambda_{2 \gamma}+\lambda_{3 \gamma}+\lambda_{4 \gamma}+\cdots
$$

from which it follows that

$$
\begin{aligned}
& \lambda\left({ }^{1} S_{0}\right)=\lambda_{2 \gamma}+\lambda_{4 \gamma}+\lambda_{6 \gamma}+\ldots \approx \lambda_{2 \gamma} \\
& \lambda\left({ }^{3} S_{1}\right)=\lambda_{3 \gamma}+\lambda_{5 \gamma}+\lambda_{7 \gamma}+\ldots \approx \lambda_{3 \gamma}
\end{aligned}
$$

where the cross-sections for $4 \gamma$ and higher annihilation processes are negligible compared to $2 \gamma$ and $3 \gamma$ annihilations. It is for these reasons that $p$-Ps self-annihilates into $2 \gamma$ while $o$-Ps must self-annihilate into $3 \gamma$.

Section 2.5.1 also discussed the angular and energy distributions for the $2 \gamma$ annihilation photons. The motion of the electron-positron pair was described in terms of a laboratory frame $X$, where the pair moved with velocity $\mathrm{v}=v_{\mathrm{L}} \hat{y}+v_{\tau} \hat{v}_{\mathrm{T}}$, and a centre-ofmass frame $X^{\prime}$, in which the pair is stationary. The photons in $X$ have frequencies $\omega_{ \pm}$and wave vectors $k_{ \pm}$which are, in general, different from those in $X^{\prime}$. However, the phase of each photon remains invariant between reference frames

$$
\phi_{ \pm}=\omega_{ \pm} t-\mathbf{k}_{ \pm} \cdot \mathbf{r}=\omega^{\prime} t^{\prime}-\mathbf{k}_{ \pm}^{\prime} \cdot \mathbf{r}^{\prime} .
$$

Using (B.4) and the usual Lorentz transformations for $(c t, \mathbf{x})$ and $\left(\omega_{ \pm} / c, \mathbf{k}_{ \pm}\right)$gives

$$
\begin{aligned}
\omega_{ \pm} & =\gamma\left(\omega^{\prime} \pm \mathbf{v} \cdot \mathbf{k}_{ \pm}^{\prime}\right) \\
k_{\mathrm{L} \pm} & =\gamma\left(k_{\mathrm{t} \pm}^{\prime} \pm \frac{v \omega^{\prime}}{c^{2}}\right) \\
\mathbf{k}_{\mathrm{\tau} \pm} & =\mathbf{k}_{\mathrm{\tau} \pm}^{\prime}
\end{aligned}
$$


where $\mathbf{k}_{ \pm}$and $\mathbf{k}_{ \pm}^{\prime}$, which have angles $\theta_{ \pm}$and $\theta_{ \pm}^{\prime}$ relative to $\mathbf{v}$, have also been separated into components parallel $k_{\mathrm{t} \pm \pm}$ and perpendicular $k_{\tau \pm}$ to $v$. For photons $\left|k_{ \pm}\right|=\omega_{ \pm} / c$ and $\left|\mathbf{k}_{ \pm}^{\prime}\right|=\omega_{ \pm}^{\prime} / c$ from which it follows immediately that

$$
\begin{aligned}
\omega_{ \pm} & =\omega^{\prime} \gamma\left(1 \pm \beta \cos \theta_{ \pm}^{\prime}\right) \\
\tan \theta_{ \pm} & =\frac{\sin \theta_{ \pm}^{\prime}}{\gamma\left(\cos \theta_{ \pm}^{\prime} \pm \beta\right)}
\end{aligned}
$$

which simply expresses the relativistic Doppler shift (Jackson 1975). From (B.6a) it follows directly that

$$
E_{ \pm}=\frac{1}{2} E_{\mathrm{tot}}\left(1 \pm \beta \cos \theta_{ \pm}^{\prime}\right)
$$

where the total energy in $X, E_{\text {tor }}=\gamma E_{\text {tot }}^{\prime}=2 m_{e} c^{2}-E_{b}+E_{e}+E_{p}$, includes the binding energy and the kinetic energy of the electron-positron pair. Assuming $v \ll c$ and ignoring small differences in the emission angles in each frame (i.e. $\theta_{ \pm}^{\prime} \approx \theta_{ \pm}$) gives (2.39) which expresses the Doppler shift. Using (B.6b), elementary trigonometric identities and again ignoring small differences in the emission angles it follows that

$$
\theta \approx \frac{2 v}{c} \sin \theta_{+} \approx \frac{2 v}{c} \sin \theta_{-} \approx \frac{p_{T}}{m_{o} c}
$$

which is the same as (2.40) and expresses the angular deviation of the $\gamma$-rays from $180^{\circ}$ in the laboratory frame.

Equation (2.41) in $\$ 2.5 .2$ gives the probability of a $2 \gamma$ annihilation event in a condensed matter system for photons having total momentum p. This result is basic to most positron annihilation studies. The following provides a cursory description of its derivation. One of the most complete theoretical treatments of positrons annihilating in an arbitrary system of electrons and external fields is that of Chang Lee (1958). In that work, a series of canonical transformations were applied to a Hamiltonian consisting of terms describing the influence of an external field on the particles $\left(\hat{\mathbf{H}}_{0}\right)$, the Coulomb interaction between particles $\left(\hat{\mathbf{H}}_{1}\right)$, and the interaction between the particles and the radiation field $\left(\hat{\mathbf{H}}^{\prime}\right)$. Under these transformations $\hat{\mathbf{H}}^{\prime}$ separates into one part which conserves the number of particles, and can be treated together with $\hat{\mathbf{H}}_{0}$ and $\hat{\mathbf{H}}_{1}$, and second part which deals with the creation and annihilation of electron-positron pairs in which the effects of the external field and the Coulomb interaction enter only through the wave functions of the system.

The part of $\hat{\mathbf{H}}^{\prime}$ responsible for $2 \boldsymbol{\gamma}$ annihilations, $\hat{\mathbf{H}}_{2 \gamma}^{\prime}$, is of second order. The reason is that each interaction of an electron or a positron with the radiation field can create only 
one photon. The $2 \gamma$ annihilation of an electron-positron pair is therefore a second-order process that must proceed via an intermediate state $|I\rangle$, as shown in the Feynman diagrams in Table 2.1. In the intermediate state, either the electron or the positron has emitted a photon with $p=m_{0} c$ and is recoiling with approximately the same momentum ${ }^{\dagger}$. The recoiling virtual particle then annihilates with its partner producing a second photon. Since $\hat{\mathbf{H}}^{\prime}$ is a perturbation to $\hat{\mathbf{H}}_{0}+\hat{\mathbf{H}}_{1}$ the probability, per unit volume in momentum space, of going from an initial state $|i\rangle$ ( $n$ electrons, one positron) to any of the possible final states $|f\rangle(n-1$ electrons, 2 photons) with total photon-pair momentum $\hbar \mathrm{q}$ is

$$
\rho_{2 \gamma}(\mathbf{q}) \propto \sum_{f}\left|\left\langle f\left|\hat{\mathbf{H}}_{2 \gamma}^{\prime}\right| i\right\rangle\right|^{2}
$$

where

$$
\left\langle f\left|\hat{\mathbf{H}}_{2 \gamma}^{\prime}\right| i\right\rangle=\sum_{I} \frac{\left\langle f\left|\dot{\mathrm{H}}_{2 \gamma}^{\prime}\right| I\right\rangle\left\langle I\left|\hat{\mathrm{H}}_{2 \gamma}^{\prime}\right| i\right\rangle}{E_{i}-E_{I}} .
$$

Slow electron-positron pairs have an energy $E_{i} \ll E_{I}$ which implies that the energy denominator in (B.9b) is essentially constant and so the completeness relation can be used to sum over the intermediate states.

Using second-quantization notation, Ferrell (1956) showed that $\hat{\mathbf{H}}_{2 \gamma}^{\prime}$ is proportional to

$$
\dot{\mathbf{H}}_{2 \gamma}^{\prime} \propto \sum_{\mathbf{q}_{1}} \sum_{\mathbf{q}_{2}} a_{\mathbf{q}_{1}} b_{\mathbf{q}_{2}} \delta_{\mathbf{q}_{1}+\mathbf{q}_{2}, \mathbf{q}}
$$

where $a_{\mathbf{q}_{1}}$ and $b_{\mathbf{q}_{2}}$ are the plane wave annihilation operators, in momentum representation, for the electron and positron, respectively, and the $\delta$-function conserves momentum. These annihilation operators can be written in terms of their corresponding position field operators $\dot{\psi}_{e}(\mathbf{x})$ and $\hat{\psi}_{p}(\mathbf{x})$

$$
\begin{aligned}
& a_{\mathbf{q}_{1}}=\frac{1}{\sqrt{V}} \int e^{-i \mathbf{q}_{1} \cdot \mathbf{x}} \dot{\psi}_{e}(\mathbf{x}) d \mathbf{x} \\
& b_{\mathbf{q}_{2}}=\frac{1}{\sqrt{V}} \int e^{-i \mathbf{q}_{2} \cdot \mathbf{x}} \dot{\psi}_{\mathbf{p}}(\mathbf{x}) d \mathbf{x}
\end{aligned}
$$

where $V$ is the normalization volume and $\mathbf{x}$ represents both spatial and spin coordinates. Inserting (B.11) into (B.10) and summing over $q_{1}$ and $q_{2}$ gives

$$
\hat{\mathbf{H}}_{2 \gamma}^{\prime} \propto \int e^{-i q \cdot \mathbf{x}} \hat{\psi}_{e}(\mathbf{x}) \hat{\psi}_{p}(\mathbf{x}) d \mathbf{x}
$$

\footnotetext{
$\dagger_{\text {The energy of }}|I\rangle$ is $E_{I} \approx m_{0} c^{2}$ so it can exist only for $\Delta t \leq \frac{1}{2} \frac{n}{m_{0} c^{2}} \approx 10^{-21}$ s. In this time the virtual particle travels $\Delta x \leq \frac{1}{2} \frac{n}{m_{o} c} \approx 10^{-13} \mathrm{~m}$, which implies that the annihilating $\mathrm{e}^{-}-\mathrm{e}^{+}$pair is 'in contact' on an atomic scale.
} 
which can be inserted into (B.9a) to give

$$
\rho_{2 \gamma}(\mathbf{q})=\frac{\pi r_{0}^{2} c}{(2 \pi)^{3}} \iint e^{-i \mathbf{q} \cdot(\mathbf{x}-\mathbf{y})}\left\langle i\left|\dot{\psi}_{e}^{\dagger}(\mathbf{y}) \bar{\psi}_{e}(\mathbf{x}) \tilde{\psi}_{p}^{\dagger}(\mathbf{y}) \hat{\psi}_{p}(\mathbf{x})\right| i\right) d \mathbf{x} d \mathbf{y}
$$

where the completeness equation has been used to sum over the final states and an appropriate choice for the multiplying constant has been made. Equation (B.13) is simply the probability per unit time per unit volume in momentum space that an electron-positron annihilation produces two photons with total momentum $\hbar \mathbf{q}$. A simple evaluation of (B.13) results in the following expression in configuration space

$$
\rho_{2 \gamma}(\mathbf{q})=\frac{\pi r_{o}^{2} c}{(2 \pi)^{3}} N \int d \mathbf{r}_{1} \ldots \int d \mathbf{r}_{N-1}\left|\int e^{-i \mathbf{q} \cdot \mathbf{r}} \Psi\left(\mathbf{r}_{1} \ldots \mathbf{r}_{N-1}, \mathbf{r}, \mathbf{r}\right) d \mathbf{r}\right|^{2}
$$

where $\Psi\left(r_{1} \ldots r_{N}, r_{p}\right)$ is an initial one positron, $N$ electron wave function that is properly antisymmetric in all the electron coordinates averaged over spin directions. Chang Lee (1958) showed that if the many-body wave function is written as the product of a positron wave function $\Psi_{p}\left(r_{p}\right)$ and a Slater determinant of $N$ one electron wave functions $\Psi_{e, i}\left(r_{e}\right)$ then (B.14) reduces to the IPM result given by (2.50), assuming that the annihilation did not change the wave functions of the remaining $N-1$ electrons. When $\Psi\left(\mathbf{r}_{1} \ldots \mathbf{r}_{N}, \mathbf{r}_{p}\right)$ is written in terms of a two-particle electron-positron wave function $\Psi_{e p, i}\left(r_{e}, r_{p}\right)$ the momentum density is given by (2.41), which was our starting point for the discussion of positron annihilation in condensed matter. 

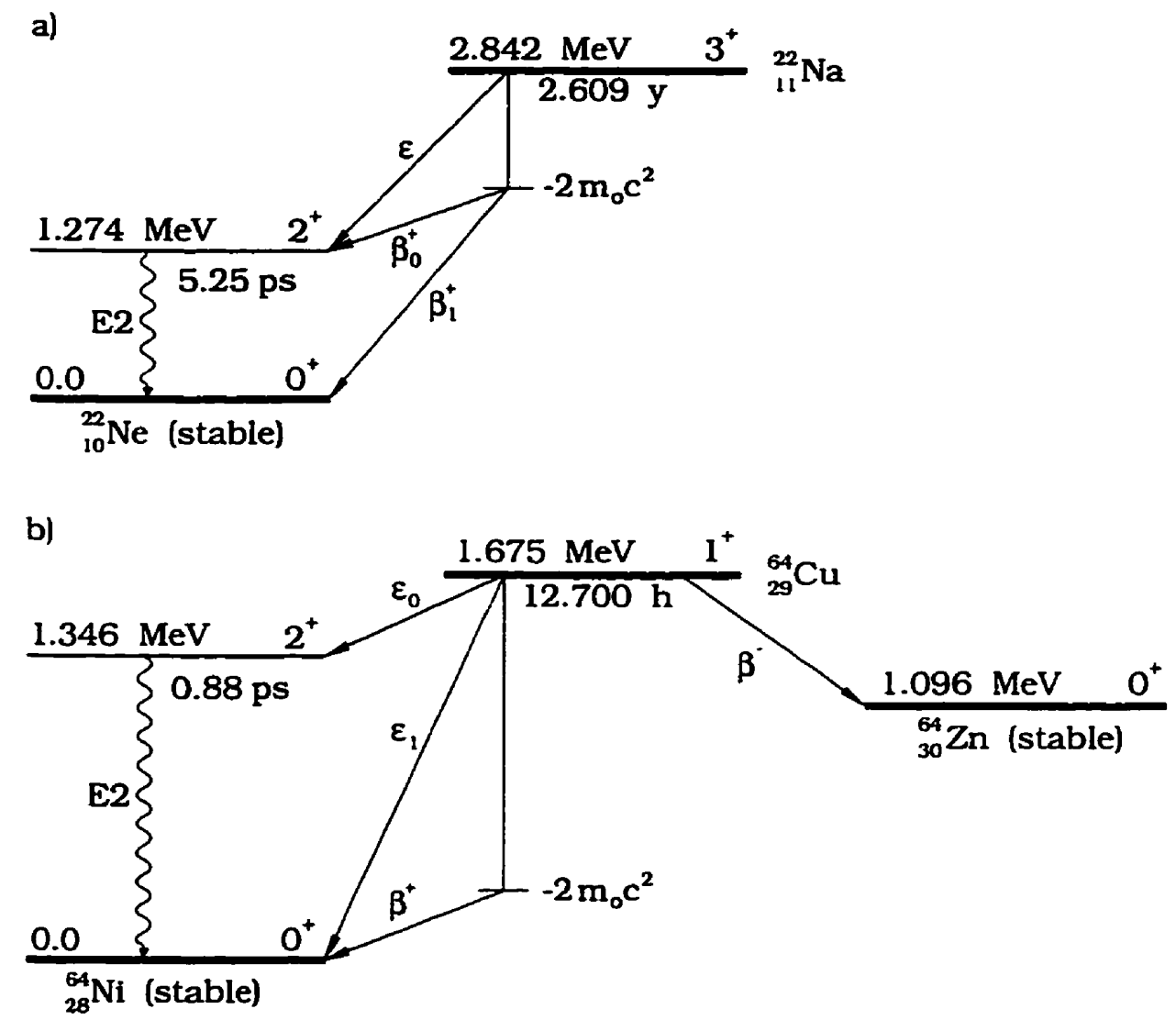

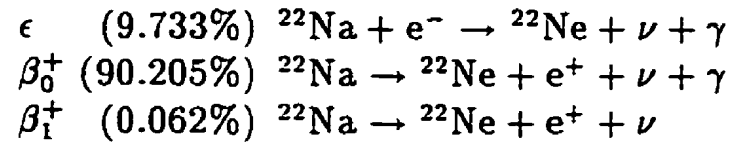

$$
\begin{aligned}
& \epsilon_{0}(0.471 \%){ }^{64} \mathrm{Cu}+\mathrm{e}^{-} \rightarrow{ }^{64} \mathrm{Ni}+\nu+\gamma \\
& \epsilon_{1}(42.6 \%){ }^{64} \mathrm{Cu}+\mathrm{e}^{-} \rightarrow{ }^{64} \mathrm{Ni}+\nu \\
& \beta^{+}(17.86 \%) \\
& { }^{64} \mathrm{Cu} \rightarrow{ }^{64} \mathrm{Ni}+\mathrm{e}^{+}+\nu \\
& \beta^{-}(39.0 \%){ }^{64} \mathrm{Cu} \rightarrow{ }^{64} \mathrm{Zn}+\mathrm{e}^{-}+\bar{\nu}
\end{aligned}
$$

Figure B.1: Decay modes for ${ }^{22} \mathrm{Na}$ and ${ }^{64} \mathrm{Cu}$. a) The ${ }^{22} \mathrm{Na}$ state exists $2.842 \mathrm{MeV}$ above the ${ }^{22} \mathrm{Ne}$ ground state. When ${ }^{22} \mathrm{Na} \beta$-decays a virtual state, consisting of the original nucleus plus a virtual electron-positron pair, is produced at $1.82 \mathrm{MeV}\left(2 m_{o} c^{2}\right.$ below the $3^{+}$state). The electron is absorbed by a proton producing ${ }^{22} \mathrm{Ne}$ while the positron and a neutrino are emitted. The majority of $\beta$-decays are to the first excited state of ${ }^{22} \mathrm{Ne}$, in which case the positron and neutrino share $0.54 \mathrm{MeV}$ and a $1.27 \mathrm{MeV} \gamma$-ray is emitted about 5 ps after the positron. In positron lifetime experiments this $\gamma$-ray is used to mark the birth of the positron. About $10 \%$ of the decays go by electron capture and do not produce a positron. b) The $\beta^{+}$-decay of ${ }^{64} \mathrm{Cu}$ is similar to that of ${ }^{22} \mathrm{Na}$ except that now the virtual state is below the first excited state of ${ }^{64} \mathrm{Ni}$ so no prompt $\gamma$-ray is produced. A significant fraction of the decays proceed by electron capture and $\beta^{-}$-decay and therefore do not produce positrons. 


\section{Appendix C}

\section{Thermalization Times in the Condensed Rare Gases}

In $\S 2.3$ the question was raised of whether or not a positron was fully thermalized prior to annihilation in condensed insulating materials. In all condensed media, inelastic scattering brings the original high-energy positron down to tens of $\mathrm{eV}$ in a few picoseconds. This will be shown to be the case below. In metals, inelastic scattering is efficient all the way down to a fraction of an $\mathrm{eV}$. However, in the condensed rare gases the large band gaps ensure that inelastic processes have a threshold at $\sim 5-10 \mathrm{eV}$. Once positrons fall below this threshold they can only thermalize by the rather inefficient phonon scattering in solids or by static and long-wavelength density fluctuations in liquids. The main concern is therefore whether or not a positron with several eV of kinetic energy can thermalize prior to annihilation. This question has not been satisfactorily answered in insulating materials.

The condensed rare gases, with their large band gaps and lack of optical-phonon or vibrational modes, are expected to provide the most severe test of positron thermalization. Thermalization times for both electrons and Ps have been estimated experimentally. Sowada et al. (1982a, 1982b) measured the time for electrons with an average kinetic energy of $\sim 10 \mathrm{eV}$ to reach thermal energies in the heavier condensed rare gases. The thermalization times, given in Table C.1, are on the order of nanoseconds - a time that is significantly longer than in other insulators ${ }^{\dagger}$. Despite having an electron mobility similar to that for argon, the thermalization time in methane is a factor of 50 smaller - a direct result of the possibility of exciting molecular vibrational and rotational modes in methane. A further decrease to less than a picosecond is found as the molecular complexity increases. They also noticed that long-range order did not play an important role in the exchange of energy or momentum between electrons and the condensed rare gas matrices - that particle density alone appears to be the decisive factor. Tuomisaari et al. (1991) measured the energy distributions of Ps emitted from the rare-gas solids following positron implantation. Fitting the results of simulations to their data they deduced that Ps was typically formed with an

\footnotetext{
†Onn and Silver (1971) and Smejtek et al. (1973) have injected hot electrons into liquid $\mathrm{He}, \mathrm{H}_{2}$ and $\mathrm{N}_{2}$ and found that these electrons slow down to $\sim 1 \mathrm{eV}$ on the order of a picosecond. Liquid $\mathrm{He}, \mathrm{Ne}$ and $\mathrm{H}_{2}$ have low electron mobilities and fast thermalization times due to electron bubble formation, while in liquid $\mathrm{N}_{2}$ it is due to the formation of a temporary anion state.
} 
Table C.1: Thermalization times for electrons with an average initial kinetic energy of $\sim 10 \mathrm{eV}$ in weakly scattering heavier condensed rare gases. Sowada et al. (1982a, 1982b) measured the transient conductivity resulting from a high energy radiation pulse. Since the electron mobility is inversely proportional to the square root of the thermal electron velocity, the slowing down of electrons increases the drift mobility and the conductivity. The thermalization time was defined as the rise time of the conductivity signal from 10 to $90 \%$. These measurements are in agreement with values estimated from deformation-potential theory (see below) for the interactions of electrons with acoustic phonons. The result for methane is an estimate based on the same theory.

\begin{tabular}{lcc}
\hline \hline Medium & T (K) & Thermalization times (ps) \\
\hline $\mathrm{Ar}(\mathrm{s})$ & 82 & $500 \pm 400$ \\
$\mathrm{Ar}(\mathrm{l})$ & 85 & $900 \pm 200$ \\
$\mathrm{Kr}(\mathrm{s})$ & 113 & $2200 \pm 200$ \\
$\mathrm{Kr}(\mathrm{l})$ & 117 & $4400 \pm 200$ \\
$\mathrm{Xe}(\mathrm{s})$ & 157 & $4400 \pm 400$ \\
$\mathrm{Xe}(\mathrm{l})$ & 163 & $6500 \pm 500$ \\
$\mathrm{CH}_{4}(\mathrm{l})$ & 111 & 22 \\
\hline \hline
\end{tabular}

average initial kinetic energy less than one $\mathrm{eV}$ and that it thermalized in less than 10 ps. Their results, shown in Table C.4, are consistent with those of Kubica and Stewart (1975) who showed that free Ps slowed down to as low as $10_{-5}^{+2} \mathrm{~K}$ in single crystal quartz. For the case of positrons in solid argon, Rose and DeBenedetti (1965) remarked that there was no evidence to indicate they were not thermalized. In the following, positron thermalization times will be estimated to try to determine whether they are more like those of electrons or Ps.

In general, the time $t$ for a particle of mass $m$ to slow down from an initial energy $T_{i}$ to a final energy $T_{f}$ is given by (Brandt and Arista 1982)

$$
t=-\sqrt{\frac{m}{2}} \int_{T_{\imath}}^{T_{t}} \frac{d T}{S \sqrt{T}}
$$

where $S=-d T / d x$ is the stopping power which gives the energy loss of a particle per unit distance travelled. Equation (C.1) is based on the continuous-slowing-down approximation (csda) in which energy loses are assumed to occur almost continuously in small bits through interactions with the medium. This is obviously a somewhat different description of the slowing-down process than that given in $\S 2.3$. The uncertainties associated with the csda can be found in the abundant literature on slowing down of electrons in radiation physics 
and chemistry.

The stopping power describing the energy transfer from high-energy electrons and positrons to bound atomic electrons has been calculated using the Bethe-Bloch type formulas derived from the Møller and Bhabha cross sections for electron-electron and positronelectron scattering, respectively (Rohrlich and Carlson 1954). It can be written as

$$
S_{\mathrm{e}, \pm}=\frac{0.153536}{\beta^{2}} \frac{Z \varrho}{A} B_{ \pm}(T) \quad\left(\frac{\mathrm{MeV}}{\mathrm{cm}}\right)
$$

where $B_{ \pm}(T)$ is the stopping number, $T$ is the particle's kinetic energy, $v$ is its speed, $\beta=v / c, \varrho$ is the density of the medium in $\mathrm{g} / \mathrm{cm}^{3}$ and $Z / A$ is the ratio of the atomic number to atomic weight. The stopping number is given by

$$
B_{ \pm}(T)=B_{0, \pm}(T)-2 \ln \left(I /\left(m_{o} c^{2}\right)\right)-\delta
$$

where

$$
\left.B_{o,-}(T)=\ln \left(\tau^{2}(\tau+2) / 2\right)\right)+\frac{1+\tau^{2} / 8-(2 \tau+1) \ln 2}{(\tau+1)^{2}}
$$

for electron, while for positrons

$$
\left.B_{o,+}(T)=\ln \left(\tau^{2}(\tau+2) / 2\right)\right)+2 \ln 2-\frac{\beta^{2}}{12}\left(23+\frac{14}{\tau+2}+\frac{10}{(\tau+2)^{2}}+\frac{4}{(\tau+2)^{3}}\right)(C
$$

where $\tau=T /\left(m_{0} c^{2}\right)$. $I$ is the mean excitation and ionization energy of the medium and $\delta$ is a density-effect correction ${ }^{\dagger}$. The slowing-down time is obtained by inserting $S_{\mathrm{e},+}$ into (C.1) and then integrating numerically. Brandt and Arista (1982) have done this and obtained a fit to (C.1) which provides a reasonable estimate of the slowing-down time, assuming $T_{f} \ll T_{i}$,

$$
t_{\mathrm{e}} \approx \frac{7.7}{\varrho}\left(\frac{T_{i}}{m_{o} c^{2}}\right)^{1.2} \times 10^{-12} \mathrm{~s} .
$$

Equation (C.2), and therefore (C.5), is only valid for projectile energies significantly larger than the ionization energy of the target electrons, due to the Born approximation on which the Bethe theory is based. For most condensed media (C.5) is valid down to $\sim 1 \mathrm{keV}$ and is typically used down to a few hundred $\mathrm{eV}$. Table C.2 gives the time for positrons with initial $E_{\max }=0.65 \mathrm{MeV}$ to slow down to $1 \mathrm{keV}$ in the condensed rare gases. This is the maximum kinetic energy for a positron emitted from ${ }^{64} \mathrm{Cu}$ and is therfore an upper limit on

\footnotetext{
tSeltzer and Berger (1984) list values for $I$ and $\delta$ for a wide range of materials. $\delta=0$ for projectile energies below several tens of $\mathrm{MeV}$ in the rare gases.
} 
Table C.2: High-energy positron thermalization times in the condensed rare gases. The thermalization times were estimated using (C.4b), (C.5) and (C.6) for positrons with initial energies $E_{\max }=0.65 \mathrm{MeV}$. Equation (C.4b) was integrated down to $T_{f}=1 \mathrm{keV}$ with the required ionization potential, $I$, being taken from Seltzer and Berger (1984). Equation (C.6) was integrated all the way down to the solid band gap energy $E_{\text {gap }}$ (see Table A.5, the band gap energy for helium is $24.6 \mathrm{eV}$ ). The other binding energies required in (C.6) were taken from Schwentner et al. (1985). Below the lowest exciton formation threshold $E_{\mathrm{x}}$ positrons can only thermalize by phonon or density fluctuation scattering and Ps formation. The energy levels in liquids will be slightly different than those given below, however, the results are insensitive to small changes in the binding energies. The main difference between solid and liquid targets felt by the positron is the density (see Tables A.2 and A.3). All energies are in $\mathrm{eV}$ and times in picoseconds.

\begin{tabular}{lrrrrr}
\hline & $\mathrm{T}(\mathrm{K})$ & $I$ & $t(\mathrm{C} .4 \mathrm{~b})$ & $t(\mathrm{C} .5)$ & $t(\mathrm{C} .6)$ \\
\hline $\mathrm{He}^{4}(\mathrm{l})$ & 4.2 & 41.8 & 65 & 82 & 74 \\
$\mathrm{Ne}(\mathrm{s})$ & 24.6 & 137 & 7 & 7 & 7 \\
$\mathrm{Ne}(\mathrm{l})$ & 27.1 & & 8 & 9 & 9 \\
$\mathrm{Ar}(\mathrm{s})$ & 83.8 & 188 & 7 & 6 & 8 \\
$\mathrm{Ar}(\mathrm{l})$ & 87.3 & & 8 & 7 & 9 \\
$\mathrm{Kr}(\mathrm{s})$ & 116.0 & 352 & 4 & 4 & 5 \\
$\mathrm{Kr}(\mathrm{l})$ & 119.8 & & 5 & 4 & 6 \\
$\mathrm{Xe}(\mathrm{s})$ & 161.4 & 482 & 4 & 3 & 4 \\
$\mathrm{Xe}(\mathrm{l})$ & 165.1 & & 5 & 4 & 5 \\
\hline \hline
\end{tabular}

the slowing-down times. Positrons starting with $E_{\text {ave }}=0.28 \mathrm{MeV}$ have slowing-down times that are 2-3 times shorter than those in Table C.2. The slowing-down times calculated from (C.4b) and (C.5) are in reasonable agreement and, except for helium, are always less than 10 ps. This is very short compared to the positron lifetime $(\sim 0.4 \mathrm{~ns})$ so that virtually all positrons survive down to below a $\mathrm{keV}$.

A second approach to finding the slowing-down time, which is useful below a $\mathrm{keV}$, is based on the semiempirical expressions of Gryzinski (1965a, 1965b, 1965c) which describe the inelastic scattering cross sections of projectiles on target atoms. Valkealahti and Nieminen $(1983,1984)$ have used these cross sections in Monte Carlo simulations of positrons slowing down in metals with some success. From Gryzinski's excitation function for the inelastic scattering of positrons and electrons one can derive an expression for the mass stopping power similar to (C.2) except that now the stopping number

$$
B_{ \pm}(T)=\sum_{i} \frac{n_{i}}{Z}\left(\frac{x_{i}-1}{x_{i}+1}\right)^{3 / 2}\left(\ln x_{i}+\frac{4}{3} \ln \left(2.7+\sqrt{x_{i}-1}\right)\right)
$$


where $n_{i}$ is the number of electrons in the $i^{\text {th }}$ shell, $x_{i}=T / E_{b, i}$ and $E_{b, i}$ is the binding energy of the electrons in the $i^{\text {th }}$ sheil. Equation (C.6) does not account for those inelastic collisions which have an energy loss lower than the binding energy. The difference between (C.2) and (C.6) is therefore a crude measure of the energy losses due to excitations (excitons. etc. $)^{\dagger}$. Table C.2 gives the thermalization times calculated using (C.6) for energies down to the lowest binding energy. As expected, these times are a little longer due almost entirely to the lower stopping powers, as the time for a positron to slow down from $1 \mathrm{keV}$ to $E_{\text {gap }}$ is negligible.

In solids, the final approach to thermal equilibrium for either a positron, or an energetic Ps, is determined by phonon scattering. The general form of the positron-phonon interaction is well known (McMullen 1985) and simple approximations typically describe it in terms of the deformation-potential approximation. In this approximation, the medium is treated as an elastic continuum by assuming that only the long-wavelength longitudinal. acoustic-phonons are important. The overall positron-phonon coupling strength is described in terms of one parameter, the deformation potential $E_{\mathrm{d}}$, which gives the change in positron energy per unit dilation of the crystal. Using this model, the energy-loss rate $d T / d t$ of positrons, and Ps, to phonon scattering in a crystal with one atom per unit cell has been given as (Conwell 1967; Tuomisaari et al. 1989)

$$
\frac{d T}{d t}=-\frac{m E_{\mathrm{d}}^{2}}{16 \pi \hbar \varrho} \frac{q_{\max }^{4}}{k}
$$

where the approximation $T=\hbar^{2} k^{2} / 2 m$ is used. The acoustic phonons are treated in the Debye model and thus have energies $\hbar \omega_{q}=\hbar s q$, where $s$ is the speed of sound, and a cutoff at the Debye wavevector $q_{D}=\left(6 \pi^{2} / \Omega_{0}\right)^{1 / 3}$, where $\Omega_{0}$ is the unit cell volume. The Debye wavevector corresponds to the shortest wavelength phonon that can propagate within the crystal. $q_{\max }$ is the smaller of $2 k$ and $q_{D}$. Using (C.7) the phonon stopping power is easily obtained

$$
S_{\mathrm{ph}}=\frac{m E_{\mathrm{d}}^{2} q_{\mathrm{D}}^{4}}{32 \pi \varrho} \frac{1}{T}
$$

for $k>q_{D} / 2$, while for $k<q_{D} / 2$

$$
S_{\mathrm{ph}}=\frac{2 m^{3} E_{\mathrm{d}}^{2}}{\pi \hbar^{4} \varrho} T
$$

\footnotetext{
'Doke et al. (1976) have calculated ratios of the number of excitations to the number of ionizations of $0.21,0.10$ and 0.06 for high-energy electrons passing through liquid $\mathrm{Ar}, \mathrm{Kr}$ and $\mathrm{Xe}$, respectively. The difference between (C.2) and (C.6) is similar to these results. They also measured an average energy loss per ionization of $23.6 \pm 0.3$ and $15.6 \pm 0.03 \mathrm{eV}$ for electrons in liquid Ar and Xe, respectively.
} 
These equations are easily integrated to give the stopping times

$$
t_{\mathrm{ph}}=\frac{32 \sqrt{2} \pi \varrho}{3 \sqrt{m} E_{\mathrm{d}}^{2} q_{D}^{4}}\left(T_{i}^{3 / 2}-T_{D}^{3 / 2}\right)
$$

where $T_{D}=\left(\hbar^{2} / 2 m\right)\left(q_{D} / 2\right)^{2}$ and $T_{D}<T_{i}$, and

$$
t_{\mathrm{ph}}=\frac{\pi \hbar^{4} \varrho}{\sqrt{2} m^{5 / 2} E_{\mathrm{d}}^{2}}\left(\frac{1}{\sqrt{T_{f}}}-\frac{1}{\sqrt{T_{D}}}\right)
$$

for $T_{f}<T_{D}$. Equations (C.8a) and (C.9a) neglect Umklapp scattering, which is possible above $T_{D}$, and may therefore underestimate the stopping power and overestimate the stopping time, respectively.

In general, the equations described above are valid only for solids. Basak and Cohen (1979) have developed a semiphenomenological theory of electron mobility in liquid insulators. In their model, an excess electron is in quasi-free state in the conduction band of the liquid and is occasionally scattered by a deformation potential produced by longwavelength fluctuations of the liquid density. Their results reproduce the electron mobility results in fluid argon quite nicely over a wide range of fluid densities. The results of Sowada et al. (1982a, 1982b) indicate that the deformation-potential approach is equally well applicable to the rare-gas liquids near the triple point as well as to solids despite the loss of long-range order. Furthermore, they have shown that this approach is applicable in the condensed rare gases despite the usual condition for application of the basic theoretical equations being that the electron (positron, Ps) kinetic energy be less than the deformation potential. Based on this, the deformation-potential model will be applied to both liquids and solids, with the idea that in liquids $E_{\mathrm{d}}$ describes the coupling between the positron and long-wavelength density fluctuations.

The deformation potential required in (C.8) and (C.9) is a parameter that is either obtained from experiment or estimated from theory. In metals, $E_{\mathrm{d}}$ is typically on the order of $-2 E_{F} / 3$, where $E_{F}$ is the Fermi energy (Bergersen et al. 1974). In semiconductors and polar materials optical phonons also contribute and so $E_{\mathrm{d}}$ is usually somewhat larger in absolute value $(10-20 \mathrm{eV})$. To date, there are no estimates of $E_{\mathrm{d}}$ for positrons in the condensed rare gases. The deformation potential is defined as

$$
E_{\mathrm{d}}=\Omega_{o} \frac{\partial E}{\partial \Omega_{0}}
$$

which is easily calculated using (2.31) and (2.32) from Stott's pseudopotential theory. The estimates for $E_{\mathrm{d}}$, in Table C.3, are considerably lower than are typically found in metals 
Table C.3: Low-energy positron thermalization times in the condensed rare gases. The initial energies $T_{i}$ were taken to be approximately equal to the lower Ore gap boundary. The final energy $T_{f}$ was arbitrarily set to $0.1 \mathrm{eV}$. All energies are in $\mathrm{eV}$ and times in nanoseconds.

\begin{tabular}{lrcccccc}
\hline \hline & $\mathrm{T}(\mathrm{K})$ & $q_{D}\left(\AA^{-1}\right)$ & $T_{i}$ & $T_{D}$ & $E_{\mathrm{d}}$ & $t(\mathrm{C} .9 \mathrm{a})$ & $t(\mathrm{C} .9 \mathrm{~b})$ \\
\hline $\mathrm{He}^{4}(\mathrm{l})$ & 4.2 & 1.04 & 18 & 1.03 & 0.13 & 5.9 & 0.35 \\
$\mathrm{Ne}(\mathrm{s})$ & 24.6 & 1.38 & 17 & 1.80 & -0.43 & 1.8 & 0.41 \\
$\mathrm{Ne}(\mathrm{l})$ & 27.1 & 1.29 & 17 & 1.57 & -0.40 & 2.4 & 0.40 \\
$\mathrm{Ar}(\mathrm{s})$ & 83.8 & 1.13 & 11 & 1.21 & 1.23 & 0.3 & 0.05 \\
$\mathrm{Ar}(\mathrm{l})$ & 87.3 & 1.08 & 11 & 1.10 & 0.81 & 0.7 & 0.10 \\
$\mathrm{Kr}(\mathrm{s})$ & 116.0 & 1.01 & 10 & 0.96 & 1.59 & 0.4 & 0.04 \\
$\mathrm{Xe}(\mathrm{s})$ & 161.4 & 0.94 & 8 & 0.83 & 1.77 & 0.4 & 0.05 \\
\hline \hline
\end{tabular}

resulting in reduced stopping powers and therefore increased thermalization times. Notice that the estimated thermalization times for helium and neon exceed the measured positron lifetime. In argon, krypton and xenon the predicted thermalization times are comparable to the positron lifetime.

The deformation potential for energetic Ps in the heavier rare-gas solids has recently been estimated by Tuomisaari et al. (1991). They measured the energy distributions of Ps emitted from rare-gas solids following positron implantation. By fitting a model of hot Ps losing energy as it diffuses back to the surface to their data they calculated the deformation potential $E_{\mathrm{d}}$ for epithermal Ps. Their results are given in Table C.I. Ps formed in the Ore gap process is expected to have an initial kinetic energy less than its binding energy. Tuomisaari et al. (1991) found that Ps was typically formed with an average initial kinetic energy less than $1 \mathrm{eV}$. Using their estimates for $E_{\mathrm{d}}$ and the initial energy in (C.9b) gives times of less than $5 \mathrm{ps}$, which is very short compared to the Ps lifetime. Using these deformation potentials, even if the Ps is assumed to start with an initial energy equal to its own binding energy, the thermalization times are still less than about 10 ps. It is therefore reasonable to conclude that $P$ s does reach thermal equilibrium long before it annihilates. 
Table C.4: Positronium thermalization times in the condensed rare gases. These results are taken from Tuomisaari et al. (1991) who measured the energy distributions of Ps emitted from solid $\mathrm{Ar}, \mathrm{Kr}$ and $\mathrm{Xe}(30-40 \mathrm{~K})$ following positron implantation at incident energies of $0.1-2 \mathrm{keV}$. Using a model of hot Ps losing energy as it diffuses back to the surface, they deduced the average Ps kinetic energy at formation $T_{\text {ave,Ps, }}$, the average Ps band energy $T_{i}$, the deformation potential $E_{\mathrm{d}}$ for epithermal Ps, the thermalization time (calculated using (C.9b) with an effective mass of $2 m_{o}$, since the Ps energy is below the Umklapp scattering threshold) and a range of mean free times $t_{s c}$ between collision during slowing down. The thermalization and the mean free times presented below are a factor of 2 smaller than those calculated by Tuomisaari et al. (1991) who mistakenly used equations derived in Tuomisaari et al. (1989) for crystals with 2 atoms per unit cell.

\begin{tabular}{cccccc}
\hline & $T_{\text {ave,Ps }}(\mathrm{eV})$ & $T_{\mathrm{i}}(\mathrm{eV})$ & $E_{\mathrm{d}}(\mathrm{eV})$ & $t(\mathrm{C} .9 \mathrm{~b})(\mathrm{ps})$ & $t_{\mathrm{sc}}(\mathrm{fs})$ \\
\hline $\mathrm{Ar}$ & 0.91 & 0.45 & 6 & 5.2 & $1-10$ \\
& & & 8 & 2.9 & $0.5-5$ \\
$\mathrm{Kr}$ & 0.57 & 0.50 & 8 & 5.1 & $0.5-8$ \\
& & & 10 & 3.3 & $0.5-5$ \\
$\mathrm{Xe}$ & 0.50 & 0.35 & 10 & 4.0 & $0.5-5$ \\
& & & 14 & 2.0 & $0.5-3$ \\
\hline
\end{tabular}




\section{Appendix D}

\section{Extended Self-Trapped Positron States}

In this appendix, the possibility of a positron existing in an extended self-trapped state is considered. Leung et al. (1976) have developed a simple model to predict if the self-trapping of positrons by lattice distortions can occur. The model does not however provide an adequate description of the self-trapped state. What follows is a brief summary of their model followed by its application to the rare-gas solids.

As was done in the previous appendix, the lattice is treated as an elastic continuum which interacts with the positron via a deformation potential, $E_{d}$. The discrete nature of the lattice is partially accounted for by restricting the wavevectors of the acoustic phonons to be greater than the Debye wavevector, $q_{D}$. The energy functional for the positron self-trapped in a dilation $\Delta(\mathbf{r})$ in the continuum is

$$
E\left[\Psi_{p}(\mathbf{r}, \Delta(\mathbf{r})]=\frac{B}{2} \int \Delta^{2}(\mathbf{r}) d \mathbf{r}+E_{d} \int\left|\Psi_{p}(\mathbf{r})\right|^{2} \Delta(\mathbf{r}) d \mathbf{r}-\frac{\hbar^{2}}{2 m_{p}^{*}} \int \Psi_{p}^{*}(\mathbf{r}) \nabla^{2} \Psi_{p}(\mathbf{r}) d \mathbf{r}\right.
$$

where $B$ is the bulk modulus, $\Psi_{p}$ is the positron wave function and $m_{p}^{*}$ is its effective mass. By minimizing (D.1) with respect to $\Delta(\mathbf{r})$ gives an expression for $\Delta(\mathbf{r})$ which can be substituted back into (D.1) to obtain a variational energy as a functional of $\Psi_{p}$ alone. Using a trial positron wave function of the form

$$
\Psi_{p}(\mathbf{r})=(2 \beta)^{3 / 4} e^{-\pi \beta^{2} r^{2}}
$$

gives the variational energy as

$$
E(\beta)=\frac{3 \pi \hbar^{2} \beta^{2}}{2 m_{p}^{*}}-\frac{E_{d}^{2}}{4 \pi B} \int_{0}^{q_{D}} q^{2} \exp \left(\frac{-q^{2}}{4 \pi \beta^{2}}\right) d q
$$

where $\beta$ is a variational parameter. A minimum in (D.3) for $\beta>0$ implies that a selftrapped state is possible. A minimum variational energy at $\beta=0$ describes a completely delocalized positron state.

Using the data in Tables A.5 and C.3 the coupling constants $\left(E_{d}^{2} / B\right)$ for solid $\mathrm{Ne}$, $\mathrm{Ar}, \mathrm{Kr}$ and $\mathrm{Xe}$ were calculated to be $27,89,116$ and $139 \mathrm{eV} \AA^{3}$, respectively. Using these results, the value of $\beta$ corresponding to the minimum of (D.3) for the rare-gas solids was 
calculated. Using $m_{p}^{*}=m_{o}$ the minimum energy occured at $\beta=0$ in all cases. This describes a completely delocalized positron and implies that self-trapping does not occur. The coupling constants would have to be several times higher before extended self-trapping would even begin to occur. 


\section{Appendix E}

\section{Positronium Spin-Exchange Cross-Sections}

The purpose of this appendix is to calculate the relative spin-exchange cross-sections for Ps colliding with species having either one or two unpaired electrons. Both of these situations have been discussed to some extent by Ferrell (1958) and Wallace (1960). To begin, consider an elastic collision between Ps and a species having only one unpaired electron, such as a spur electron. The cross-sections for transitions between Ps states are expressed in terms of the amplitude for a direct elastic collision $D_{\mathfrak{l}}$, where no electron interchange occurs, and $E_{1}$ which is the amplitude for collisions with electron exchanges. Symbolically these transition matrix elements may be written as

$$
\begin{aligned}
& D_{1}=\left\langle\phi\left(\mathbf{r}_{1}\right) \Psi_{\mathrm{Ps}_{\mathbf{s}}}\left(\mathbf{r}_{e}, \mathbf{r}_{p}\right)\left|V_{\mathrm{l}}\right| \phi\left(\mathbf{r}_{\mathrm{l}}\right) \Psi_{\mathrm{Ps}_{\mathbf{s}}}\left(\mathbf{r}_{e}, \mathbf{r}_{p}\right)\right\rangle \\
& E_{1}=\left\langle\phi\left(\mathbf{r}_{\mathrm{l}}\right) \Psi_{\mathrm{P}_{\mathbf{s}}}\left(\mathbf{r}_{e}, \mathbf{r}_{p}\right)\left|V_{1}\right| \phi\left(\mathbf{r}_{e}\right) \Psi_{\mathrm{Ps}_{\mathbf{s}}}\left(\mathbf{r}_{\mathrm{l}}, \mathbf{r}_{p}\right)\right\rangle
\end{aligned}
$$

where $\Psi_{P s}$ is the spatial part of the Ps ground state wave function, $\Phi$ is the spatial part of the unpaired electron species and $V_{1}$ is an interaction potential between them. The subscripts $(1, e)$ on the electron coordinates are used to simply clarify the interactions. The Ps spin wave functions are given by (3.4), while those for $\phi$ are simply $|\uparrow\rangle$ and $|\downarrow\rangle$. Collisions with the $S_{!}^{1}$ Ps triplet state give

$$
\begin{aligned}
\left|\uparrow_{1}\right\rangle+\left|\uparrow_{e} \neq\right\rangle & \rightarrow D_{1}\left(\left|\uparrow_{1}\right\rangle+\left|\uparrow_{e} \uparrow\right\rangle\right)-E_{1}\left(\left|\uparrow_{e}\right\rangle+\left|\uparrow_{1} \uparrow\right\rangle\right) \\
& =\left(D_{1}-E_{1}\right)\left(|\uparrow\rangle+S_{1}^{1}\right)\left|\downarrow_{1}\right\rangle+\left|\uparrow_{e} \uparrow\right\rangle \rightarrow D_{1}\left(\left|\downarrow_{1}\right\rangle+\left|\uparrow_{e} \neq\right\rangle\right)-E_{1}\left(\left|\uparrow_{e}\right\rangle+\left|\downarrow_{1} \uparrow\right\rangle\right) \\
& =D_{1}\left(|\downarrow\rangle+S_{1}^{1}\right)-\frac{E_{1}}{\sqrt{2}}\left(|\uparrow\rangle+S_{0}^{1}\right)+\frac{E_{1}}{\sqrt{2}}\left(|\uparrow\rangle+S_{0}^{0}\right)
\end{aligned}
$$

where the Pauli principle introduces a negative sign with the exchange of same spin electrons. Similar results hold for the $S_{-1}^{1}$ state. Collisions with the $S_{0}^{1}$ state gives

$$
\begin{aligned}
\left|\uparrow_{1}\right\rangle+\frac{1}{\sqrt{2}}\left(\left|\uparrow_{e} \neq\right\rangle\right. & \left.+\left|\downarrow_{e} \neq\right\rangle\right) \rightarrow \frac{D_{1}}{\sqrt{2}}\left(\left|\uparrow_{e} \downarrow\right\rangle+\left|\downarrow_{e} \neq\right\rangle\right)-\frac{E_{1}}{\sqrt{2}}\left(\left|\uparrow_{e}\right\rangle+\left|\uparrow_{1} \downarrow\right\rangle\right)+\frac{E_{1}}{\sqrt{2}}\left(\left|\downarrow_{e}\right\rangle+\left|\uparrow_{1} \neq\right\rangle\right) \\
& =\left(D_{1}-\frac{E_{1}}{2}\right)\left(|\uparrow\rangle+S_{0}^{1}\right)-\frac{E_{1}}{2}\left(|\uparrow\rangle+S_{0}^{0}\right)+\frac{E_{1}}{\sqrt{2}}\left(|\downarrow\rangle+S_{1}^{1}\right) .
\end{aligned}
$$

Similar equations hold for $|\downarrow\rangle+S_{0}^{\perp}$ and for collisions with the singlet p-Ps state $S_{0}^{0}$. The cross-sections for transitions between Ps states are obtained by squaring the appropriate 
amplitudes and averaging over each unpaired electron states. Doing this gives

\begin{tabular}{c|cccc} 
& $S_{0}^{0}$ & $S_{-1}^{\mathrm{l}}$ & $S_{0}^{1}$ & $S_{1}^{\mathrm{l}}$ \\
\hline$S_{0}^{0}$ & $D_{1}^{2}-D_{\mathrm{1}} E_{\mathrm{1}}+\frac{1}{4} E_{1}^{2}$ & $\frac{1}{4} E_{1}^{2}$ & $\frac{1}{4} E_{1}^{2}$ & $\frac{1}{4} E_{1}^{2}$ \\
$S_{-1}^{1}$ & $\frac{1}{4} E_{1}^{2}$ & $D_{1}^{2}-D_{1} E_{1}+\frac{1}{2} E_{1}^{2}$ & $\frac{1}{4} E_{1}^{2}$ & 0 \\
$S_{0}^{1}$ & $\frac{1}{4} E_{1}^{2}$ & $\frac{1}{4} E_{1}^{2}$ & $D_{1}^{2}-D_{1} E_{1}+\frac{1}{4} E_{1}^{2}$ & $\frac{1}{4} E_{1}^{2}$ \\
$S_{1}^{1}$ & $\frac{1}{4} E_{1}^{2}$ & 0 & $\frac{1}{4} E_{1}^{2}$ & $D_{1}^{2}-D_{1} E_{1}+\frac{1}{2} E_{1}^{2}$
\end{tabular}

Notice that the amount of $p$-Ps $\rightarrow o$-Ps is compensated by the the conversion of $o-P s \rightarrow$ $p$-Ps. If the conversion rate, which is proportional to the cross-section (i.e. $E_{1}^{2}$ ), is significantly faster than $\lambda_{1}^{o}$, or significantly slower than $\lambda_{3}^{o}$, then the ratio of $p$-Ps to $o$-Ps is expected to remain $\sim \frac{1}{4}: \frac{3}{4}$. However, if the conversion rate is comparable to the annihilation rates then the $p$-Ps fraction increases as some of it is able to annihilate before converting. The ratio of the quenching to scattering cross-sections for $p$-Ps and $o$-Ps are

$$
\begin{aligned}
& \frac{\sigma_{1}(p \rightarrow o)}{\sigma_{1}(p \rightarrow p)}=\frac{\frac{3}{4} E_{1}^{2}}{D_{1}^{2}-D_{1} E_{1}+\frac{1}{4} E_{1}^{2}} \\
& \frac{\sigma_{1}(o \rightarrow p)}{\sigma_{1}(o \rightarrow o)}=\frac{\frac{1}{4} E_{1}^{2}}{D_{1}^{2}-D_{1} E_{1}+\frac{3}{4} E_{1}^{2}}
\end{aligned}
$$

respectively.

The above results are easily modified to account for spin-exchange conversions in a magnetic field. In this case, the Ps eigenstates are given by $S_{ \pm 1}^{1},|-\rangle=\frac{1}{\sqrt{2}}(\alpha|\uparrow \downarrow\rangle-\beta|\downarrow \neq\rangle)$ and $|+\rangle=\frac{1}{\sqrt{2}}(\beta|\uparrow \downarrow\rangle+\alpha|\downarrow \uparrow\rangle)$ where $\alpha=(1-y) / \sqrt{1+y^{2}}$ and $\beta=(1+y) / \sqrt{1+y^{2}}$ with $y$ defined by (3.53). The relative cross-sections for transitions between Ps states are now

\begin{tabular}{c|cccc} 
& $|-\rangle$ & $S_{-1}^{1}$ & $|+\rangle$ & $S_{1}^{1}$ \\
\hline$|-\rangle$ & $D_{1}^{2}-D_{1} E_{1}+\frac{1}{2} E_{1}^{2}$ & $\frac{1}{4} \alpha^{2} E_{1}^{2}$ & $\frac{1}{4} \alpha^{2} \beta^{2} E_{1}^{2}$ & $\frac{1}{4} \beta^{2} E_{1}^{2}$ \\
$S_{-1}^{1}$ & $\frac{1}{4} \alpha^{2} E_{1}^{2}$ & $D_{1}^{2}-D_{1} E_{1}+\frac{1}{2} E_{1}^{2}$ & $\frac{1}{4} \beta^{2} E_{1}^{2}$ & 0 \\
$|+\rangle$ & $\frac{1}{4} \alpha^{2} \beta^{2} E_{1}^{2}$ & $\frac{1}{4} \beta^{2} E_{1}^{2}$ & $D_{1}^{2}-D_{1} E_{1}+\frac{1}{2} E_{1}^{2}$ & $\frac{1}{4} \alpha^{2} E_{1}^{2} \quad(E .6)$ \\
$S_{1}^{1}$ & $\frac{1}{4} \beta^{2} E_{1}^{2}$ & 0 & $\frac{1}{4} \alpha^{2} E_{1}^{2}$ & $D_{1}^{2}-D_{1} E_{1}+\frac{1}{2} E_{1}^{2}$
\end{tabular}

These results were first derived by Bisi et al. $(1981,1987)$ who used them in rate equations to describe the spin-exchange conversions of Ps on paramagnetic molecules and ions in aqueous solutions. In this work, they are used to describe the spin-exchange conversion rates in a magnetic field for Ps collisions on spur electrons.

Finally, we are interested in elastic and inelastic spin-exchange collisions of Ps with the oxygen molecule. The $\mathrm{O}_{2}$ ground state, denoted by $\mathrm{X}^{3} \Sigma_{g}^{-}$, is a triplet state with two unpaired electrons. For elastic collisions $D_{2}$ and $E_{2}$ are similar to (E.1) except that now 
$\phi\left(\mathbf{r}_{1}, \mathbf{r}_{2}\right)$ is the spatial part for a species with two unpaired electrons. As before, crosssections for the conversion between Ps states is calculated by squaring the amplitudes obtained for collisions between each of the four Ps spin states and the three $\mathrm{O}_{2}$ triplet spin states and then averaging over the $\mathrm{O}_{2}$ states (Wallace 1960). The elastic scattering cross-sections are

\begin{tabular}{|c|c|c|c|c|}
\hline & $S_{0}^{0}$ & $S_{-1}^{1}$ & $S_{0}^{1}$ & $S_{1}^{1}$ \\
\hline$S_{0}^{0}$ & $D_{2}^{2}-2 D_{2} E_{2}+E_{2}^{2}$ & $\frac{2}{3} E_{2}^{2}$ & $\frac{2}{3} E_{2}^{2}$ & $\frac{2}{3} E_{2}^{2}$ \\
\hline$S_{-1}^{1}$ & $\frac{2}{3} E_{2}^{2}$ & $D_{2}^{2}-2 D_{2} E_{2}+\frac{5}{3} E_{2}^{2}$ & $\frac{2}{3} E_{2}^{2}$ & 0 \\
\hline$S_{0}^{\mathrm{I}}$ & $\frac{2}{3} E_{2}^{2}$ & $\frac{2}{3} E_{2}^{2}$ & $D_{2}^{2}-2 D_{2} E_{2}+E_{2}^{2}$ & $\frac{2}{3} E_{2}^{2}$ \\
\hline$S_{1}^{\mathrm{t}}$ & $\frac{2}{3} E_{2}^{2}$ & 0 & $\frac{2}{3} E_{2}^{2}$ & $D_{2}^{2}-2 D_{2} E_{2}+\frac{5}{3} E_{2}^{2}$ \\
\hline
\end{tabular}

In this case, the ratio of the quenching to scattering cross-sections for $p$-Ps and $o$-Ps are

$$
\begin{aligned}
& \frac{\sigma_{2}(p \rightarrow o)}{\sigma_{2}(p \rightarrow p)}=\frac{\left.2 E_{2}^{2}\right)}{\left(D_{2}-E_{2}\right)^{2}} \\
& \frac{\sigma_{2}(o \rightarrow p)}{\sigma_{2}(o \rightarrow o)}=\frac{\frac{2}{3} E_{2}^{2}}{D_{2}^{2}-D_{2} E_{2}+\frac{7}{3} E_{2}^{2}}
\end{aligned}
$$

respectively.

Assuming that the Ps has enough kinetic energy, spin-exchange collisions which leave $\mathrm{O}_{2}$ in an excited state are also possible. The two lowest excited states of $\mathrm{O}_{2}$ are the singlet states $\mathrm{a}^{1} \Delta_{g}$ and $\mathrm{b}^{\mathfrak{i}} \Sigma_{g}^{+}$which lie $0.977 \mathrm{eV}$ and $1.627 \mathrm{eV}$ above $\mathrm{X}^{3} \Sigma_{g}^{-}$, respectively. Equation (E.7) has omitted those amplitudes which leave $\mathrm{O}_{2}$ in a singlet spin state. Kakimoto et al. $(1987,1990)$ have shown that in low-density $\mathrm{O}_{2}$ gas these inelastic spin-exchange collisions are also important. In this case, the transition matrix elements are

$$
\begin{aligned}
& E_{2 a}=\left\langle\phi_{a}\left(\mathbf{r}_{i}, \mathbf{r}_{2}\right) \Psi_{\mathrm{Ps}_{s}}\left(\mathbf{r}_{e}, \mathbf{r}_{p}\right)\left|V_{2}\right| \phi_{a}\left(\mathbf{r}_{2}, \mathbf{r}_{e}\right) \Psi_{\mathrm{Ps}_{s}}\left(\mathbf{r}_{1}, \mathbf{r}_{p}\right)\right\rangle \\
& E_{2 b}=\left\langle\phi_{b}\left(\mathbf{r}_{1}, \mathbf{r}_{2}\right) \Psi_{\mathbf{P s}_{s}}\left(\mathbf{r}_{e}, \mathbf{r}_{p}\right)\left|V_{2}\right| \phi_{b}\left(\mathbf{r}_{2}, \mathbf{r}_{e}\right) \Psi_{\mathbf{P s}_{s}}\left(\mathbf{r}_{1}, \mathbf{r}_{p}\right)\right\rangle
\end{aligned}
$$

where $\phi_{a}$ and $\phi_{b}$ are the spatial parts of the $a^{1} \Delta_{g}$ and $b^{1} \Sigma_{g}^{+}$wave functions, respectively. The inelastic scattering cross-sections (i.e. those which leave $\mathrm{O}_{2}$ in a spin singlet state) are

\begin{tabular}{c|cccc} 
& $S_{0}^{0}$ & $S_{-1}^{\mathrm{1}}$ & $S_{0}^{1}$ & $S_{1}^{1}$ \\
\hline$S_{0}^{0}$ & 0 & $\frac{1}{3} E_{2}^{2}$ & $\frac{1}{3} E_{2}^{2}$ & $\frac{1}{3} E_{2}^{2}$ \\
$S_{-1}^{1}$ & $\frac{1}{3} E_{2}^{2}$ & $\frac{1}{3} E_{2}^{2}$ & $\frac{1}{3} E_{2}^{2}$ & 0 \\
$S_{0}^{1}$ & $\frac{1}{3} E_{2}^{2}$ & $\frac{1}{3} E_{2}^{2}$ & 0 & $\frac{1}{3} E_{2}^{2}$ \\
$S_{1}^{1}$ & $\frac{1}{3} E_{2}^{2}$ & 0 & $\frac{1}{3} E_{2}^{2}$ & $\frac{1}{3} E_{2}^{2}$
\end{tabular}

Equation (E.10) is correct for $\mathrm{X}^{3} \Sigma_{g}^{-} \rightarrow \mathrm{b}^{1} \Sigma_{g}^{+}$. However, for $\mathrm{a}^{1} \Delta_{g} \Lambda$-doubling gives two closely spaced levels which means that each cross-section in (E.10) should be multiplied 
by two for $\mathrm{X}^{3} \Sigma_{g}^{-} \rightarrow \mathrm{a}^{1} \Delta_{g}$. Kakimoto et al. (1990) have experimentally estimated the cross-sections for inelastic and elastic $o$-Ps $\rightarrow p$-Ps conversions in low-density $\mathrm{O}_{2}$ gas to be $\sim 5 \times 10^{-17} \mathrm{~cm}^{2}$ and $(1.0 \pm 0.3) \times 10^{-19} \mathrm{~cm}^{2}$, respectively. Generally, one would expect the elastic cross-section to be on the order of atomic dimensions (i.e. $10^{-16} \mathrm{~cm}^{2}$ ) with the quenching cross-sections being an order of magnitude or so lower. However, this does not appear to be the case for $\mathrm{O}_{2}$ whose elastic cross-section is significantly lower than expected. To date, no theoretical estimates exist for either of these cross-sections. 


\section{Appendix F \\ The 1D-ACAR Experimental Resolution}

The main contribution to the experimental resolution function, $R\left(\theta_{z}\right)$, comes from the finite widths of the collimating slits in front of the two detectors. Assuming a point source and convoluting two slits of width $w$ gives this contribution to the geometrical resolution, $g_{1}\left(\theta_{z}\right)$. The result is a unit area triangle with a FWHM equal to the angle subtended by the detector slits

$$
g_{1}\left(\theta_{z}\right)= \begin{cases}\frac{1}{\theta_{w}}\left(1-\left|\theta_{z}\right| / \theta_{w}\right), & \left|\theta_{z}\right|<\theta_{w} \\ 0, & \left|\theta_{z}\right|>\theta_{w}\end{cases}
$$

where $\theta_{w}=w / L$ is the angular width of the slits and $L=12.70 \mathrm{~m}$ is the sample to detector distance.

A second contribution to the geometrical resolution, $g_{2}\left(\theta_{z}\right)$, arises from the finite sample thickness and the positron implantation profile. The sample thickness $t$ is defined by the width of the collimating slits nearest to the sample. The experimental setup used two positron sources, typically separated by $2-3 \mathrm{~mm}$, placed on either side of the defined sample and shielded from view of the detectors. The positron implantation profile from each source was exponential, as described by (2.1). The sum of these exponentials results in a positron intensity seen by the detectors that is reasonably constant. Assuming infinitely thin detector slits and a constant positron intensity the response function due to a finite sample thickness is simply a rectangle of base width $2 \theta_{t}$ and height $1 /\left(2 \theta_{t}\right)$, where $\theta_{t}=t / L$. The total geometric resolution is a convolution of $g_{1}\left(\theta_{z}\right)$ and $g_{2}\left(\theta_{z}\right)$ the result of which is well approximated by a Gaussian function (see (4.7)) with unit area and a FWHM of $\theta_{g}=\sqrt{\theta_{w}^{2}+\left(2 \theta_{t}\right)^{2}}$.

The total resolution function is obtained by convoluting the geometric resolution with a Maxwell-Boltzmann distribution, which is assumed to describe the positrons' thermal motion. The Maxwell-Boltzmann distribution, $f\left(\theta_{z}, T\right)$ where $\theta_{z} \approx p_{z} /\left(m_{0} c\right)$, is also a unit area Gaussian with a FWHM $\theta_{f}=2 \sqrt{2 \ln 2 m^{*} k_{B} T} /\left(m_{o} c\right)$. The effective positron mass is approximated by simply letting $m^{*}=m_{0}$. The result of convoluting the two Gaussians is 
another unit area Gaussian having a FWHM

$$
\begin{aligned}
\theta_{R} & =\sqrt{\theta_{g}^{2}+\theta_{f}^{2}} \\
& =\sqrt{\theta_{u}^{2}+\left(2 \theta_{\imath}\right)^{2}+\left(9.333 \times 10^{-4}\right) T}
\end{aligned}
$$

where $\theta_{R}, \theta_{w}$, and $\theta_{t}$ are all in milliradians. 


\section{Appendix G \\ The Löwdin Alpha-Function Expansion}

In the building the crystal potentials in $§ 5.3 .1$ it was necessary to include the effects of the neighbouring atomic potentials and charge densities. This is accomplished using a technique, described by Löwdin (1956), in which a function associated with one centre $O_{R \Theta \Phi}$ is expanded in terms of spherical harmonics $Y_{l m}$ of another centre $O_{r \theta \phi}$. Consider a point $P$ which has coordinates $(r, \theta, \phi)$ and $(R, \Theta, \Phi)$ with respect to origins $O_{r \theta \phi}$ and $O_{R \Theta \Phi}$, respectively, where the polar axis of the two coordinate systems has been chosen along the same line but with opposite direction for the sake of symmetry. It immediately follows that

$$
\begin{gathered}
R^{2}=a^{2}+r^{2}-2 a r \cos \theta, \Phi=\phi \\
R \cos \Theta+r \cos \theta=a, R \sin \Theta=r \sin \theta
\end{gathered}
$$

where $a$ is the distance between origins. Now we want to expand a function centred at $O_{R \Theta \Phi}$

$$
F_{L M}(R, \Theta, \Phi)=f_{L}(R) Y_{L M}(\Theta, \Phi)
$$

in terms of spherical harmonics centred at $O_{r \theta \phi}$

$$
F_{L M}(R, \Theta, \Phi)=\sum_{l=0}^{\infty} \sum_{m=-l}^{+l} \alpha(l m \mid L M) Y_{l m}(\theta, \phi)
$$

where the $\alpha(l m \mid L M)$ are the expansion coefficients. Multiplying both sides of (G.3) by $Y_{l^{\prime} m^{\prime}}(\theta, \phi)$ and integrating over $d \Omega_{\theta \phi}$ gives

$$
\alpha(l m \mid L M)=\int Y_{l m}^{\ddot{m}}(\theta, \phi) F_{L M}(R, \Theta, \Phi) d \Omega_{\theta \phi}
$$

the expansion coefficients which have been tabulated by Löwdin (1956).

For the purpose of constructing the crystal potentials a very simple form of (G.3) can be used. There are two reasons for this. First, the functions to be expanded are spherically symmetric so that $L=M=0$ which means (G.2) becomes

$$
F_{00}(R, \Theta, \Phi)=\frac{1}{\sqrt{4 \pi} f_{0}(R)}
$$


Secondly, only the spherically symmetric contributions from the neighbouring atoms can be included in the central muffin-tin. This means that only the $l=m=0$ terms survive in sum in (G.3) and so

$$
\alpha(00 \mid 00)=\frac{1}{4 \pi} \int f_{0}(R) d \Omega_{\theta \phi} .
$$

From (G.1) we see that $R$ does not depend on $\phi$ so integration over this variable simply gives $2 \pi$. The remaining integration over $\theta$ can be done using (G.1) and a change of variables $(\sin \theta d \theta=R d R /(a r))$ which gives

$$
f_{0}(a, r)=\alpha(00 \mid 00)=\frac{1}{2 a r} \int_{|a-r|}^{a+r} R f_{0}(R) d R .
$$

This result gives the spherically symmetric contribution at $r$ as measured from $O_{\text {reo }}$, due to the function $f_{0}(R)$ centred at $O_{R \Theta \Phi}$ where the two origins are separated by distance $a$. 


\section{Appendix $\mathrm{H}$ \\ Calculation of the Solid-State Potentials}

The APW calculations require that the solid-state potentials be approximated by muffin-tin potentials of the form described by (2.13). The potential is assumed to be spherically symmetric inside the muffin-tin spheres centred at each atomic site and is constant between the spheres. The APW method gives accurate solutions to the Schrödinger equation for this approximate potential. The quality of the results, however, depends on how closely this potential approximates the actual crystal potential. In present work, the muffin-tin potentials were constructed using a technique first suggested by Mattheiss (1964). This technique provides a sufficiently accurate model of the actual potential and which has been used successfully in many applications, including electronic calculations in the rare-gas solids.

The correctness of the APW calculations was tested by calculating electron potentials and band structure in the rare-gas solids and then comparing them to previous results. The total potential seen by an electron in a crystal can be written as

$$
V_{e}(\mathbf{r})=V_{\text {Coul }}(\mathbf{r})+V_{\mathrm{xc}}(n(\mathbf{r}))
$$

where $V_{\text {Coul }}(\mathbf{r})$ is the Coulomb potential due to the nuclei and the crystalline charge density $n(\mathbf{r})$ and $V_{\mathrm{xc}}$ is the exchange-correlation potential. A particularly simple approximation for $V_{\text {Coul }}$ and $n(\mathbf{r})$ is obtained by superimposing atomic potentials and charge densities

$$
V_{\text {Coul }}(\mathbf{r})=\sum_{\nu} v_{\text {Coul }}\left(\left|\mathbf{r}-\mathbf{R}_{\nu}\right|\right) \quad n(\mathbf{r})=\sum_{\nu} n_{\mathrm{at}}\left(\left|\mathbf{r}-\mathbf{R}_{\nu}\right|\right)
$$

where $\mathbf{R}_{\nu}$ runs over all occupied atomic sites, and $v_{\text {Coul }}$ and $n_{\text {at }}$ are the atomic Coulomb potential and electron density, respectively. In the inner region of each muffin-tin sphere the potential and charge density are predominantly atomic in character and therefore spherically symmetric. However, in the outer portion of a muffin-tin the contributions from neighbouring atoms become important and remove the required spherical symmetry. To overcome this difficulty Mattheiuss (1964) used the Löwdin alpha-function expansion, described in Appendix G, which included only the spherically symmetric contributions from the neighbouring atoms. Within a particular muffin-tin sphere, say the $j^{\text {th }}$ one, the Coulomb 
potential can be written as

$$
V_{\text {Coul }}(\rho)=v_{\text {Coul }}(\rho)+\sum_{v \neq j} v_{\text {Coul }}\left(a_{i}, \rho\right)
$$

where $\rho=\mathbf{r}-\mathbf{R}_{j}$ and $a_{i}=\left|\mathbf{R}_{\nu}-\mathbf{R}_{j}\right|$ is the distance between the centres of the $\nu^{\text {th }}$ and $j^{\text {th }}$ muffin-tin spheres. Using (G.7) $v_{\text {Coul }}\left(a_{\nu}, \rho\right)$ is

$$
v_{\text {Coul }}\left(a_{\nu}, \rho\right)=\frac{1}{2 a_{\nu} \rho} \int_{\left|a_{\nu}-\rho\right|}^{a_{\nu}+\rho} R v_{\text {Coul }}(R) d R
$$

which describes the spherically symmetric contributions to the Coulomb potential at $\rho$, as measured from $\mathbf{R}_{j}$, due to the atom at $\mathbf{R}_{\nu}$. In the rare-gas solids the occupied atomic wave functions are well localized and the lattice spacings are large enough that the Coulomb potentials do not overlap appreciably. As a result, only the nearest neighbours contribute significantly to sum in (H.3).

The atomic Coulomb potential required in (H.3) has the form

$$
v_{\text {Coul }}(r)=-\frac{e^{2}}{4 \pi \epsilon_{o}} \frac{Z}{r}+U_{o}(r)
$$

where the first term describes the Coulomb attraction between an electron and a nucleus of charge $Z$ and $U_{o}(r)$ accounts for the repulsion between an electron and the unperturbed electron densities of the atom. $U_{o}(r)$ is obtained from Poisson's equation

$$
\nabla^{2} U_{o}(r)=-\frac{n_{\mathrm{at}}(r)}{\epsilon_{o}}
$$

a numerical solution of which can be found in Loucks (1967). For filled subshells $n_{\mathrm{at}}$ is spherically symmetric and can be written as

$$
\begin{aligned}
n_{\mathrm{at}}(r) & =\sum_{n, l} \sum_{m=-l}^{+l}\left|\psi_{n l m}(r, \theta, \phi)\right|^{2} \\
& =\sum_{n, l} \frac{2(2 l+1)}{4 \pi}\left|R_{n l}(r)\right|^{2}
\end{aligned}
$$

where the sum is over the filled subshells only. The wave functions required in (H.7) are usually obtained from Hartree-Fock self-consistent-field calculations. A discussion of these calculations and a tabulation of the results for all the elements can be found in Herman and Skillman (1963). Similar relativistic calculations have been performed by Liberman et al. (1971). In the present calculations, high quality "double-zeta" analytic fits to the Hartree-Fock wave functions were used. The radial wave functions become

$$
R_{n l}(r) \approx \sum c_{\alpha \beta} \frac{(2 \alpha)^{\beta+1 / 2}}{\sqrt{(2 \beta) !}} r^{\beta-1} e^{-\alpha r}
$$


where the $c_{\alpha \beta}$ are variationally determined coefficients and $\alpha$ and $\beta$ are optimized parameters. Values for $c_{\alpha \beta}, \alpha$ and $\beta$ have been tabulated by Clementi and Roetti (1974) for $Z \leq 54$.

The electron exchange-correlation potential is usually calculated using the local density approximation (LDA), i.e. $V_{\mathrm{xc}}(n(\mathbf{r}))$ only depends on the crystalline charge density at the given point $r$. A spherically symmetric charge density for inside the muffin-tin spheres can be constructed from $n_{a t}$ using the Löwdin alpha-function expansion in a way completely analogous to that used for the Coulomb potential in (H.3). Again, the small charge density overlap and the large lattice spacing of the rare-gas solids requires that only the effects of the nearest neighbours be included. The most common exchange potential is Slater's free electron-exchange approximation (Slater 1951)

$$
V_{\mathrm{xc}, \mathrm{s}}(n(\rho))=-\frac{3 e^{2}}{4 \pi \epsilon_{\mathrm{o}}}\left(\frac{3}{8 \pi} n(\rho)\right)^{1 / 3}
$$

$V_{\mathrm{xc}}(n(\rho))$ is spherically symmetric inside the muffin-tin spheres and can be added to (H.3) to give $V_{e}(\rho)$ inside the spheres. This Slater approximation is known to overestimate the exchange effect, especially in the low density tails of atomic functions. However, the quality of the results are more than sufficient for the purpose of checking the correctness of the APW calculations.

The total potential seen by a positron in a crystal is

$$
V_{p}(\mathbf{r})=-V_{\text {Coul }}(\mathbf{r})+V_{\text {corr }}(\mathbf{r})
$$

where $V_{\text {Coul }}$ is given by (H.2). $V_{\text {corr }}$ is the total electron-positron correlation potential describing the energy lowering due to the short-range pileup of electrons around the positron. In this case, the correlation potential may be written as

$$
V_{\text {corr }}(\mathbf{r})=\sum_{\nu} v_{\text {corr }}\left(\mathbf{r}-\mathbf{R}_{\nu}\right)
$$

where $\mathbf{R}_{\nu}$ again runs over all occupied atomic sites. This allows $v_{\text {Coul }}$ and $v_{\text {corr }}$ to be combined to give a single atom potential $v_{p}$

$$
V_{p}(\mathbf{r})=\sum_{\nu} v_{p}\left(\mathbf{r}-\mathbf{r}_{\nu}\right)=\sum_{\nu}-v_{\text {Coul }}\left(\mathbf{r}-\mathbf{R}_{\nu}\right)+v_{\text {corr }}\left(\mathbf{r}-\mathbf{R}_{\nu}\right)
$$

Now the total potential in (H.12) can be calculated using an equation similar to (H.3).

The electron-positron correlations in insulators arises largely from the polarization of the atomic orbitals. The long-range behaviour of this attractive polarization interaction is 
proportional to $1 / r^{4}$ for charged particles and to $1 / r^{6}$ for neutral atoms. At small distances this correlation remains finite but its actual form is unknown. In practice, this is not important since the repulsive Coulomb potential dominates at small distances. Two different models were used to try to describe the polarization interaction. The first is due to Schrader (1979) who suggested a simple semi-empirical model described by (5.5). The $r_{0}$ in this model is a cutoff parameter which Schrader determined by fitting calculated positron scattering lengths for $\mathrm{H}$ and $\mathrm{He}$ and the calculated Ps affinity to $\mathrm{H}$ to their measured values. For the other rare-gas atoms he used an interpolation scheme based on their ionization potentials to determine $r_{0}$. Despite the rather unphysical discontinuity in the derivative of $v_{\text {corr }, S}$ at $r_{0}$ these potentials were used quite successfully in positron-rare-gas atom scattering calculations. Values for both $\alpha$ and $r_{0}$ can be found in Table H.1. A second smooth correlation potential, first used by Abarenkov and Antonova (1974), was also tried (see (5.6)). Puska and Nieminen (1992) successfully used (5.6) with $r_{1}=0.90 \AA$ to calculate the positron band structure in the rare-gas solids. In the current calculations $r_{1}$ was chosen to provide the best fit for positron band-structure calculations to experimental data.

In APW calculations, the total potential outside the muffin-tin spheres is assumed to be a constant $V_{o}$ equal to average value of the potential in this region. For the rare-gas solids the potential in the interstitial regions is quite flat making it relatively easy to determine $V_{o}$. A rather straightforward technique is to simply impose a grid over the region between a sphere and its Wigner-Seitz cell boundary and then use (H.2), and similar expressions involving either $V_{x c}$ or $V_{\text {corr }}$, to calculate the average of the potentials found at each grid point. Another common technique is to simply calculate the spherical average of the total potential between the muffin-tin and the Wigner-Seitz radius

$$
V_{o}=3 \int_{R_{\mathrm{MT}}}^{R_{W s}} \frac{V(r) r^{2}}{R_{\mathrm{WS}}^{3}-R_{\mathrm{MT}}^{3}} d r
$$


Table H.1: Parameters used in the calculation of the electron and positron-rare-gas solid potentials. The parameters $r_{0}$ and $r_{1}$ were used in (5.5) and (5.6), respectively. The values for the polarizability and the parameter $r_{0}$ are taken from Schrader (1979). The $r_{1}$ parameters below gave the best fit between calculated positron band structures and the experimental data of Gullikson et al. (1988).

\begin{tabular}{lcccc}
\hline \hline & $\mathrm{Ne}$ & $\mathrm{Ar}$ & $\mathrm{Kr}$ & $\mathrm{Xe}$ \\
\hline Lattice constant, $a(\AA)$ & 4.43 & 5.31 & 5.65 & 6.13 \\
Atomic polarizability, $\alpha\left(\AA^{3}\right)$ & 0.3946 & 1.639 & 2.481 & 4.044 \\
Schrader cutoff parameter, $r_{0}(\AA)$ & 1.01 & 1.18 & 1.25 & 1.34 \\
Abarenkov parameter, $r_{\mathrm{l}}(\AA)$ & 1.058 & 0.794 & 0.847 & 1.085 \\
\hline \hline
\end{tabular}


